\title{
A SEARCH FOR QUARK COMPOSITENESS \\ WITH THE CDF DETECTOR \\ AT THE FERMILAB COLLIDER
}

\author{
A Dissertation \\ by \\ TIMOTHY LEE HESSING
}

Submitted to the Office of Graduate Studies of

Texas A\&M University

in partial fulfillment of the requirements for the degree of

DOCTOR OF PHILOSOPHY

December 1990

Major Subject: Physics 
AAB 2026 


\title{
A SEARCH FOR QUARK COMPOSITENESS \\ WITH THE CDF DETECTOR \\ AT THE FERMILAB COLLIDER
}

\author{
A Dissertation \\ by \\ TIMOTHY LEE HESSING
}

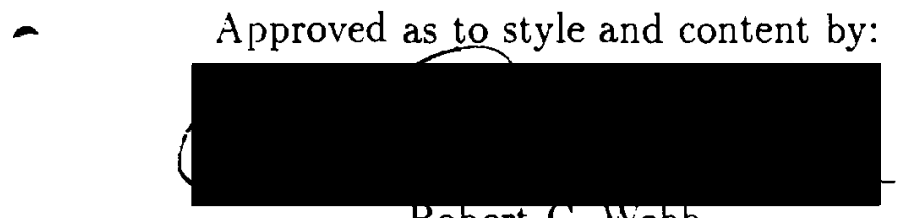

Robert C. Webb

(Chair of Committee)

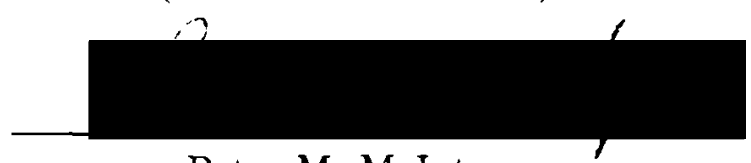

Peter M. McIntyre

(Member)

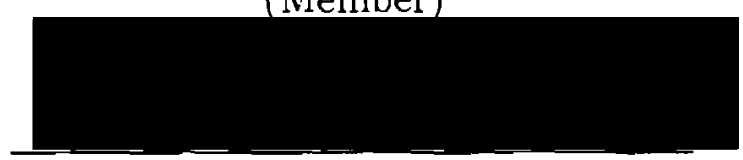

Richard L. Arnowitt

(Head of Department)

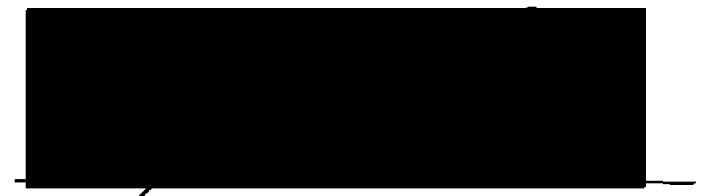

John C. Hiebert

(Member)

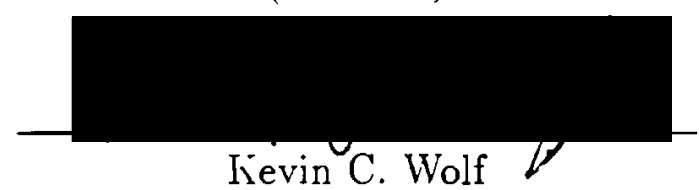

(Member) 



\section{ABSTRACT}

A Search for Quark Compositeness with the CDF

Detector at the Fermilab Collider. (December 1990)

Timothy lee Hessing, A.B., Augustana College, Rock Island, Illinois

Chair of Advisory Committce: Dr. Robert C. Webh

The inclusive jet cross section in $p \bar{p}$ collisions at $\sqrt{s}=1.8 \mathrm{TeV}$ has heren measured using jet clustering cone sizes of $0.4,0.7$ and 1.0. The cone size, $R$, is defined to be, $R=\sqrt{\Delta \eta^{2}+\Delta \phi^{2}}$, where $\eta=-\ln \tan 0 / 2,0$ represents the polar angle and $\phi$ represents the azimuthal angle. A lower limit on the value of the composite scale parameter, $\Lambda_{c}$, was determined to be $1100 \mathrm{GeV}$ at the $95 \%$ confidence level using the cone size 1.0. Comparisons to both leading order Quantum Chromo Dynamics $(Q C D)$ and next-to-leading order QCD calculations have been made and no significant deviations from QCD observed. The dependence of the inclusive jet cross section on cone size has also been compared to next-to leading order QCD. The measured cross section was found to grow larger with increasing cone size, more quickly than next-to-leading order QCD predicts.

\section{DEDICATION}

This dissertation is dedicalced to

my mother and father,

who have supported my brothers and me in all our colenvors. 


\section{ACKNOWLEDGEMENTS}

There are so many people I would like to thank including all those people listed in Appendix B, who help build and run CDF. In particular, I would like to thank Muzaffer Atac and Richard Kadel for hiring me when 1 was an undergraduate and introducing me to high energy physics and the CDF experiment. Also, I thank Rick

St. Denis who showed me around and oriented me during my first summer as a graduate student working on CDF.

I thank Robert Webb for supporting me in my studies at Texas A\&M and his patience in putting up with me. I also greatly appreciate the work of Ann David and Nick Diaczenko who built most of the forward hadron calorimeter before I became involved in the project. I also would like to thank Peter McIntyre, John Iliebert, and Kevin Wolf for agreeing to be on my committee, and thus taking on the task of reading this dissertation and giving me guidance in this regard.

I thank Teruki Kamon and more recently Themis Bowcock, for sharing with me their knowledge of high energy physics and giving me guidance in writing and working on this dissertation. 1 also thank Steve Behrends, John Iluth, Rob P'lunkett and Marjorie Slıapiro for their imnense help in increasing my understanding of high ettergy plysics, and in particular, teaching me about jets and the CDF detector.

At a personal level, I thank my fellow graduate students on CDF and at Texas A\&M, from the guys at CDF (Les, Brian, and the other guy) who went to observe the future site of the SSC with me in Texas, to my friend Phil Bowman who suffered the pains of graduate student life at Texas A\&M along witl tne
I also appreciate the patience and support of my aunt, IJelen Lindsay, who helped support my undergraduate education. I thank Pat and Tracie Summerville who allowed nee to stay with them in Collinsville on my many trips back and forth from College Station to Batavia, and still renained my friends after all the things I have broken at their house. I also thank Kevin Summerville and John Penchoff for their friendship, cven though we didn' $t$ catch any big fish in Texas when they came to visit me.

Finally, I would like to thank lynn Rinker for correcting most of my punctuation and spelling errors in this dissertation and teaching me that there is more to life than physics and footlaall 


\section{TABLE OF CONTENTS}

ABSTRACT

DEDICATION

ACKNOWLEDGEMENTS

TABLE OF CONTENTS

LIST OF TABLES

IJST OF PIGURES .

\section{CIIAPTER}

I INTRODUCTION.

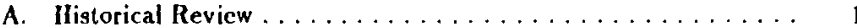

B. Experimental Status $\ldots \ldots \ldots \ldots \ldots \ldots \ldots \ldots \ldots \ldots$

C. Theoretical Motivation $\ldots \ldots \ldots \ldots \ldots \ldots \ldots \ldots \ldots, 8$

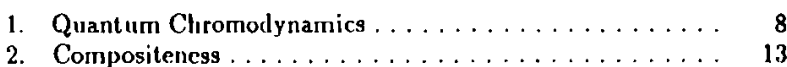

II TIIE CDF DETECTOR ................. 10

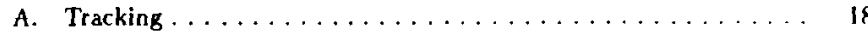

1. Vertex Time Projection Chamber . . . . . . . . 19

2. Central Tracking Chamber ............... 20

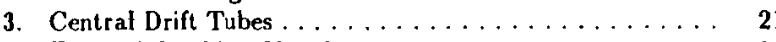

4. Forward Tracking Chamber .............. 22

B. Solenoid Magnet Coil $\ldots \ldots \ldots \ldots \ldots \ldots \ldots \ldots \ldots \ldots, \quad 22$

C. Calorimeters ........................ 23

1. Central Electromagnctic Calorimeter . . . . . . . . . . 23

2. Iladronic Central and Endwall Calorimeter . . . . . . .

3. Plug Electromagnetic Calorimeter . . . . . . . . . 25

4. Plug Lladronic Calorimeter ............... 20
('HAP'TER

Page

6. Forward Iladronic Calorimeter

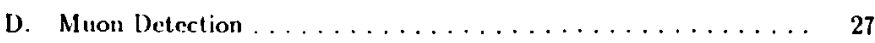

1. Central Muon Chambers ............... 27

2. Forward Muon Chambers . . . . . . . . . . . . 28

E. Data Acquisition System . . . . . . . . . . . . 29

1. Front End Electronics . . . . .

2. Data Acquisition ......................

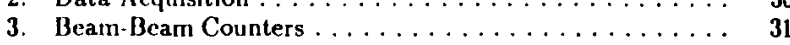

4. Triggcring .................... 32

III MEASUREMENT OF JETS AT CDF ............. 34

A. Jet Definition ...................... 34

B. Data Collection and Triggering $\ldots \ldots \ldots \ldots \ldots \ldots \ldots \ldots \ldots$

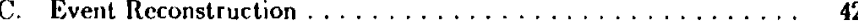

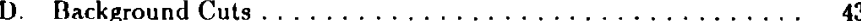

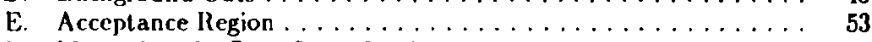

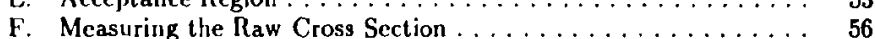

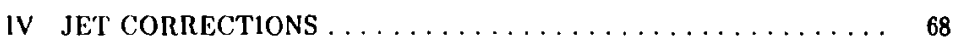

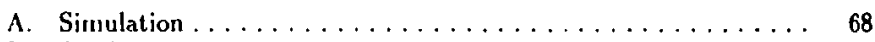

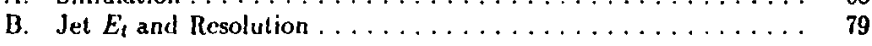

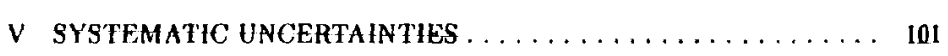

A. $E_{t}$ Scalc Systematic Uncertainties . . . . . . . . . . . . 101

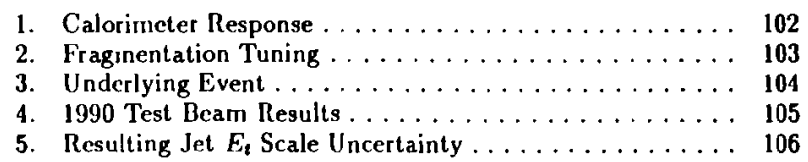

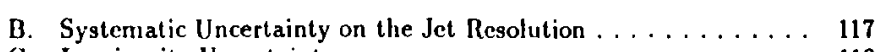

C. Luminosity Uncertainty .................... 118

D. Systentatic Uncertainty on the Jet Cross Section .......... 120

VI COMPARISONS WI'TII QCD AND A COMPOSITE MODEL ..... 131 
CIIAPTER Page

A. Filting Procedure $\ldots \ldots \ldots \ldots \ldots \ldots \ldots \ldots \ldots \ldots$

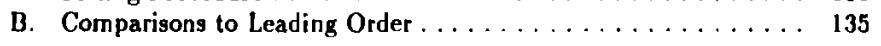

1. QCD ..................... 138

2. A Composite Model . . . . . . . . . . . . . . . . 142

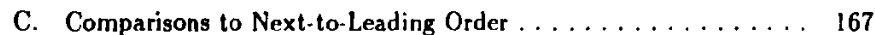

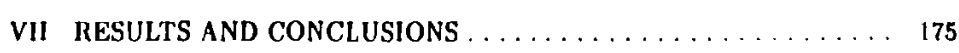

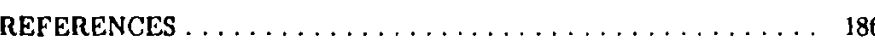

APPENDIX

A DIJET BALANCING $\ldots \ldots \ldots \ldots \ldots \ldots \ldots \ldots \ldots \ldots \ldots$

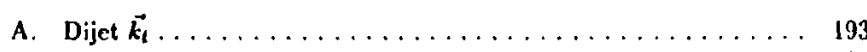

B. Jet Resolution . . . . . . . . . . . . . . . . . . 195

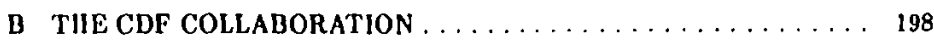

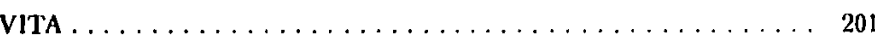




\section{LIST OF TABLES}

I. A list of the Standard Model quark and lepton fannilies and their properties. . . . . . . . . . . . . . . . . . .

II. Elementary cross scctions for leading order $\alpha_{s}^{2} Q C D$ subprocesses, given in the form $(\dot{s} / \pi) d \dot{\sigma} / d \hat{i} \ldots \ldots \ldots \ldots \ldots \ldots \ldots \ldots \ldots$

III. List of next-to-leading order $\alpha_{3}^{3}$ QCD subprocesses. . . . . . 12

IV. This is the event classification scheme for determining barkgrounds in jet events........................

V. Number of events per Class, found in the data sample used in this study. . . . . . $\ldots \ldots \ldots \ldots \ldots \ldots \ldots \ldots \ldots \ldots \ldots \ldots \ldots$

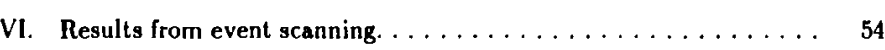

VII. Efficiency thresholds for jet triggers as a function of cone size. . . 61

VIII. Raw jet cross section and related quantities for a jet clustering cone size of 0.4, number of jets has been scaled accordingly. . . . . . 62

IX. Raw jet cross section and related quantities for a jet clustering cone size of 0.7, number of jets has been scaled accordingly. . . . . . 64

X. Raw jel cross section and related quantities for a jet clustering cone size of 1.0, number of jets has been scaled accordingly. . . . . . 66

XI. Values of the tuned parameters in SETPRT. . . . . . . . . . 74

XHI. The values of the parameters obtained for the parameterization of the Mean, for each cone size. . . . . . . . . . . . .

XIII. The values of the parameters obtained for the parameterization of the Sigma, for each cone size. . . . . . . . . . . . . . . . 8

XIV. The values of the parameters obtained for the parameterization of the Slope, for each cone size. . . . . . . . . . . . 85

XV. Fits to parameterized curve used in unsmearing procedure. . . . . 90
XV1. Corrections to the raw jet cross section and related quantities for a jet clustering cone size of $0.4 \ldots \ldots \ldots \ldots \ldots \ldots \ldots \ldots$

XV1l. Corrections to the raw jet cross section and related quantities for a jel clustering cone size of $0.7 \ldots \ldots \ldots \ldots \ldots \ldots \ldots$

XVIII. Corrections to the raw jet cross section and related quantities for a jet clustering cone size of $1.0 \ldots \ldots \ldots \ldots \ldots \ldots \ldots \ldots$

XIX. Calorimetcr response fractional systematic uncertainty on the corrected jet $E_{\imath}$ scale. . . . . . . . . . . . . . . . . . . . . 109

XX. Fraginentation fractional systematic uncertainties on the corrected

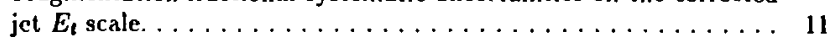

$\mathrm{XX1}$. Underlying event $E_{1}$ density fractional systematic uncertainty on the corrected jet $E_{t}$ scale. . . . . . . . . . . . . . . . . . 113

XXII. Fractional uncertainty associated with the difference in the 1990 test beam results and previous test beam results. . . . . . . 11

XXIII. Total fractional systematic uncertainty on the corrected jet $E_{t}$ scale for each cone size. . . . . . . . . . . . . . . . . . 115

XXIV. Percent systematic uncertainty on the inclusive jet cross section for a jet clustering cone size of $0.4 \ldots \ldots \ldots \ldots \ldots \ldots \ldots \ldots$

XXV. Percent systematic uncertainty on the inclusive jet cross section for a jet clustering cone size of $0.7 \ldots \ldots \ldots \ldots \ldots \ldots \ldots \ldots, 127$

XXVl. Percent systematic uncertainty on the inclusive jet cross section for a jet clustering cone size of $1.0 \ldots \ldots \ldots \ldots \ldots \ldots \ldots \ldots$

XXVII. List of the lower and upper limits defining the fitting region used

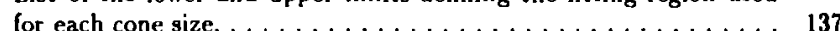

XXVIII. The $\chi^{2}$ and number of bins found in the various regions examined in the fitting procedure for a cone size of 0.4 using the various structure

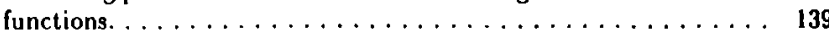

$X X I X$. The $\chi^{2}$ and number of bins found in the various regions examined in the fitting procedure for a cone size of 0.7 using the various structure functions. 
$X X X$. The $x^{2}$ and number of bins found in the various regions examined in the fitting procedure for a cone size of 1.0 using the various structure

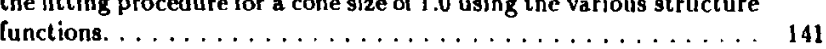

XLII. Corrected jet cross section for a jet clustering cone size of $0.4 \ldots \quad 177$

XI.III. Corrected jet cross section for a jet clustering cone size of $0.7 \ldots \quad 179$

XXXI. Combined $\chi^{2}$ (fit region combined with upper region), number of bins (including the empty bin) and the confidence level obtained using the various structure functions for a cone size of 0.4 .

XLIV. Corrected jet cross section for a jet clustering cone size of $1.0 . \ldots$ 18

XXXII. Combined $\chi^{2}$ (fit region combined with upper region), number of bins (including the empty bin) and the confidence level obtained using the various structure functions for a cone size of 0.7 . .....

XXXIII. Combined $x^{2}$ (fit region combined with upper region), number of bins (including the emply bin) and the confidence level obtained
using the various structure functions for a cone size of $1.0 \ldots \ldots, 145$

XXXIV. Confidence Levels (\%) in the fit region and upper region for a cone size of 0.4 using the various structure functions. . . . . . . $\ldots 153$

XXXV. Confidence Levels (\%) in the fit region and upper region for a cone size of 0.7 using the various structure functions. . . . . . . 154

XXXVI. Confidence Levels (\%) in the fit region and upper region for a conc size of 1.0 using the various st ructure functions. . . . . . . . . 155

XXXVII. Number of jets found in the $E_{t}$ range from 266.0 to $429.6 \mathrm{GeV}$

- number of jets predicted by theory and the significance of this excess for a cone size of $0.4 \ldots \ldots \ldots \ldots \ldots \ldots \ldots \ldots \ldots \ldots \ldots$

XXXVIII. Number of jets found in the $E_{\ell}$ range from 271.9 to $440.0 \mathrm{GeV}$, number of jets predicted by theory and the significance of this excess for a cone size of $0.7 \ldots \ldots \ldots \ldots \ldots \ldots \ldots \ldots \ldots, 164$

XXXIX. Number of jets found in the $E_{t}$ range from 273.2 to $445.6 \mathrm{GcV}$, number of jets predicted by theory and the significance of this cxcess for a cone size of 110.

XL. Number of jets over the number of events per bin found in the last six bins of the data. . ................. 166

XLI. Results of the comparison of next-to-leading order QCD calcula tions (using the structure function MRS B) of the inclusive jet cros section to the measured cross section. . . . . . . . . . . . . 


\section{LIST OF FIGURES}

1. An example of a two jet event, in the CDF detector, which illustrates a $2-2$ body $Q C D$ process. ........................ 10

2. An example of a three jet event, in the CDF detector, which illustrates a 2-3 body QCD process. . . . . . . . . . . . . . . . .

3. Perspective view of the CDF detector showing the central detector and the forward/backward detectors. . . . . . . . . . . . . . 17

4. Cut-away view of one-half of the CDF detector showing the individual detector sub-systems making up the central and forward/Lackward detectors. . . ........................ 18

5. An example of the $\eta-\phi$ segmentation in one quadrant of the CDF hadronic

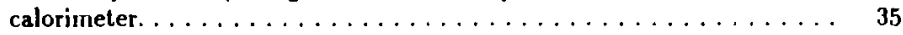

6. An illustration of a particle transversing a projective tower. . . . . . 36

7. Flow chart of the fixed cone algorithm used by CDF, starting after candidate tower, seed tower and precluster lists have been formed. . . . . . 39

8. Flow chart illustrating the reconstruction path used for the jet data. . 44

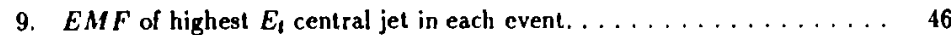

10. $E_{\ell}$ weighted $E M F$ for each event $(E M F) \ldots \ldots \ldots \ldots \ldots \ldots$

11. A verage $P_{t} / E_{\mathrm{l}}$ for all central jets in each event $(C H F) \ldots \ldots \ldots, 48$

12. Missing $E_{t}$ significance for each event $(M E T) \ldots \ldots \ldots \ldots \ldots$

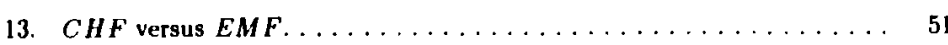

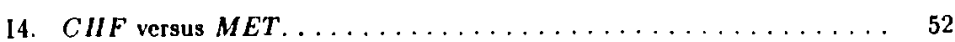

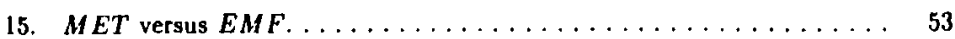

16. Effective cross section for jet backgrounds; Class 5,6 and $11 \ldots \ldots .55$

17. Fraction of jet backgrounds; Class 5,6 and $11 \ldots \ldots \ldots \ldots \ldots$

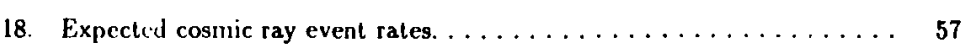

19. Event vertex distribution. . . . . . . . . . . . . . 58

20. Comparisons of the raw trigger cross sections for jets with a cone size of

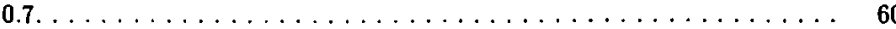

21. The distribution of $Z(T)$ in jets with a cone size of 0.7 as defined in

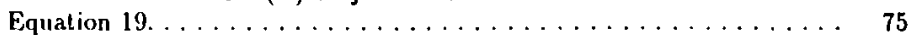

22. The jet track multiplicity in jets with a cone size of $0.7 \ldots \ldots \ldots 76$

23. The underlying event track multiplicity in jets with a cone size of 0.7. . 77

24. The distribution of $P_{\perp}$ in jets with a cone size of $0.7 \ldots \ldots \ldots \ldots$

25. Fraction of energy loss in jets with a cone size of 0.7 , due to hadronic non-linearity. . . . . . . . . . . . . .

26. The simulated response of the detector at several $E_{\mathrm{i}}$ 's for jets. . . . . 83

27. Atr illustration of the unsmearing procedure. . . . . . . . . 86

28. Unsmearing plots for cross sections of the various cone sizes. . . . . 89

29. Unsineared and corrected cross section for jets with a cone size of 0.7 , the lines indicate direction of corrections. . . . . . . . . . . . . 9

30. The 1987 cross section for jets with a cone size of 0.6 compared to $1988-$ 1989 cross section for jets with a cone size of $0.7 \ldots \ldots \ldots \ldots \ldots, 98$

31. The $1987 E_{t}$ corrections compared to the $1988-1989 E_{t}$ corrections. . . 99

32. Comparison of the rms resolution for data, simulation and the parameterized response for jets with a cone size of $0.7 \ldots \ldots \ldots \ldots \ldots \ldots, 100$

33. Loss plots as a function of measured jet $E_{t}$ for the data. . . . . 108

34. Loss plots from the simulation as a function of measured jet $E_{t} \ldots \ldots 110$

35. The fractional uncertainty of the corrected jet $E_{1}$ scale due to the difference in the 1990 test beam with previous test beanı results. . . . . . 114 
36. The breakdown of the various components of the total fractional systematic uncertainty on the corrected jet $E_{t}$ scale for earh cone size. ..... 116

37. Difference in the rms jet resolution between data and simulation. . . . 119

38. The fraction of uncertainty on the jet cross section, from the uncertainty

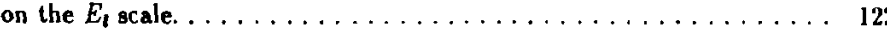

39. The fraction of uncertainty on the jet cross section, from the uncertainty

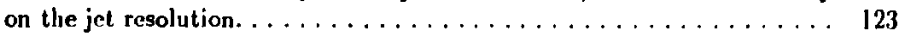

40. The total fraction of systematic uncertainty on the jet cross section. . . 124

41. Leading order QCD calculation of the inclusive jet cross section com pared to a Composite Model calculation using the structure function DFLM set 1 , with $\Lambda_{c}=950 \mathrm{GeV}$ and $Q^{2}=0.5 E_{1}^{2} \ldots \ldots \ldots \ldots 136$

42. Measured cross section compared to leading order QCD using the structure function MRS set $B$ and a cone size of $0.4 \ldots \ldots \ldots \ldots \ldots, 146$

43. Measured cross section compared to leading order QCD using the structure function MRS set 3 and cone size of $0.4 \ldots \ldots \ldots \ldots \ldots \ldots 147$

44. Measured crose section compared to leading order QCD using the structure function MRS set $B$ and a cone size of $0.7 \ldots \ldots \ldots \ldots \ldots 148$

45. Measured cross section compared to leading order $\mathrm{QCD}$ using the structure function MRS set 3 and a cone size of $0.7 \ldots \ldots \ldots \ldots \ldots 149$

46. Measured cross section compared to leading order QCD using the struc ture function MRS set $\mathrm{B}$ and a cone size of 1.0. . . . . . . 150

47. Measured cross section compared to leading order QCI) using the struc ture function MRS set 3 and a cone size of $1.0 \ldots \ldots \ldots \ldots \ldots, 15$

48. Composite calculation $\left(\Lambda_{c}=980 \mathrm{GeV}\right)$ of the inclusive jet cross section compare to the measure cross section using the structure function MRS set 2 and a cone size of $0.4 \ldots \ldots \ldots \ldots \ldots \ldots \ldots, \ldots \ldots$

49. Composite calculation $\left(\Lambda_{c}=1010 \mathrm{GeV}\right)$ of the inclusive jet cross section compare to the measure cross section using the structure function MRS set 2 and a cone size of $0.4 \ldots \ldots \ldots \ldots \ldots \ldots \ldots \ldots$
50. Composite calculation $\left(\Lambda_{c}=1050 \mathrm{GeV}\right)$ of the inclusive jet cross section compare to the measure cross section using the structure function EllLQ set 2 and a cone size of $0.7 \ldots \ldots \ldots \ldots \ldots \ldots \ldots \ldots$

51. Composite calculation $\left(\Lambda_{c}=1090 \mathrm{GeV}\right)$ of the inclusive jet cross section compare to the measure cross section using the structure function EllL, set 2 and a cone size of $0.7 \ldots \ldots \ldots \ldots \ldots \ldots \ldots \ldots \ldots$

52. Composite calculation $\left(\Lambda_{c}=1100 \mathrm{GeV}\right)$ of the inclusive jet cross section comparc to the measure cross section using the structure function EIILQ set 2 and a cone size of $1.0 \ldots \ldots \ldots \ldots \ldots \ldots \ldots \ldots$

53. Composite calculation $\left(\Lambda_{c}=1130 \mathrm{GeV}\right)$ of the inclusive jet cross section compare to the measure cross section using the structure function DFLM set 3 and a cone size of $1.0 \ldots \ldots \ldots \ldots \ldots \ldots \ldots$

54. Comparison of next-to-leading order QCD and leading order QCD, using structure function MRS set $\mathrm{B} \ldots \ldots \ldots \ldots \ldots \ldots \ldots \ldots$

55. Next-to-leading order QCD calculation of the inclusive jet cross section compare to the measure cross section using the structure function MRS B for a cone size of $0.4 \ldots \ldots \ldots \ldots \ldots \ldots \ldots \ldots \ldots \ldots \ldots$

56. Next-to-leading order QCD calculation of the inclusive jet cross section compare to the measure cross section using the structure function MRS B for a cone size of $0.7 \ldots \ldots \ldots \ldots \ldots \ldots \ldots \ldots \ldots \ldots \ldots \ldots$

57. Next-to-leading order QCD calculation of the inclusive jet cross section compare to the measure cross section using the structure function MRS B for a cone size of $1.0 \ldots \ldots \ldots \ldots \ldots \ldots \ldots \ldots \ldots$

58. Next-to-leading order QCD calculation of the inclusive jet cross section compare to the measure cross section using the structure function MRS

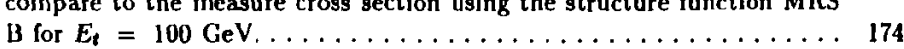

59. Inclusive jet cross section for a cone size of 0.4 , normalization uncertainty is the $E_{t}$ independent systematic uncertainty and the er ror bars represen the statistical uncertainty added in quadrature with the $\boldsymbol{E}_{\boldsymbol{t}}$ dependent

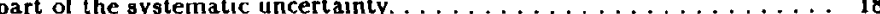


60. Inclusive jet cross section for a cone size of 0.7 , normalization uncertainty is the $E_{l}$ independent systematic uncertainty and the error bars represent the statistical uncertainty added in quadrature with the $E_{t}$ dependent part of the systematic uncertainty. . . . . . . . . . . . . . 181

61. Inclusive jet cross scction for a cone size of 1.0 , normalization uncertainty is the $E_{t}$ independent systematic uncertainty and the error bars represent is the $E_{t}$ independent systematic uncertainty and the error bars represent the statistical uncertainty added in quadrature with the $E_{t}$ dependent
part of the systematic uncertainty. $\ldots \ldots \ldots \ldots \ldots \ldots \ldots \ldots \ldots, 185$

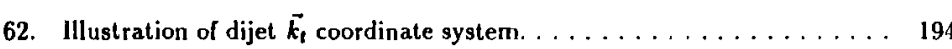

63. Dijet $\overrightarrow{k_{1}}$ distributions. . . . . . . . . . . . . . . . . . 197 


\section{CHAPTER I}

\section{INTRODUCTION}

The search for substructure in matter has led to the simplification of the phys. ical theories describing the world. At the same time it has raised more questions concerning the nature of matter. Quark substructure, or quark compositeness, is one of the next steps in this search. In this chapter, the history of this search is discussed and the current experimental state reviewed. The measurement of the inclusive jet cross section is one method of searching for quark compositeness. The theoretical justification for this measurement and its interpretation will therefore be described.

Following the theoretical motivation, the apparatus used for the measurement of jets is described in Chapter II. The measurement of the jets, the physical properties of the jets, and the determination of the inclusive jet cross section are then described in Chapter III. Chapter IV describes the calculation and use of corrections in order to compare the measured jet cross section with theory. The systematic uncertainties for this measurement are discussed in Chapter V. The cross section is compared to theory in Chapter VI, which is followed by a discussion of the results and conclusions in Chapter VII.

A. Ilistorical Review

One of the great questions in science today is the same question that philosophers asked themselves thousands of years ago, "What is the world made of ?" In This dissertation follows the style of Plysical Review D. particular, "what are the basic building blocks of matter?" Around 500 B.C., Leucippus and Democritus proposed that if one was able to break matter apart into stnaller and smaller pieces, eventually one would not be able to break matter apart any furthrr. These smallest, identical, indivisible particles were called atoms. In the carly 190 h century John Dalton composed a list of some twenty elements which he called atorns.[1] This eventually led to development of the Periodic Table of Elements, containing all the elements which are now called atoms.

In 1897 Sir J. J. Thompson discovered the electron, which was followed by Lord Rutherford's discovery of the proton in the early 1900's and, in 1932, by Sir J. Chadwick's discovery of the neutron. The atom was found to consist of these new particles and this demonstrated that the atom was not the smallest, identical, indivisible particle. In the 1960's a theory was put forth which suggested that protons and neutrons could be interpreted as composite structures. This theory, called the Gell-Mann \& Zweig Quark Model, was verified in the mid-60's by observations made at both SLAC (Stanford Linear Accelerator Center) and CERN (Conseil Européen pour la Recherche Nucléaire), which proved conclusively that the proton was made of quarks.[2]

Our current knowledge of the universe suggests that it is ruled by four forces: gravitational, electromagnetic, weak and strong. The electromagnetic and weak forces are described by the Standard Model of Electroweak Interactions. This model is based on the gauge group $S U(2) \times U(1)$. Quantum Chromodynamics (QCD) is the theory which describes the strong lorce and is based on the gauge group $S U(3)$. QCD, combined with the Standard Model of Electroweak Interactions, is known as the 
Standard Model. The Standard Model is the best thicory currently available which describes the electromagnetic, weak, and strong forces. Gravity, the weakest of the four forces, is partially described by the Theory of General Relativity. This last force has not yet been fully unified with the other three forces. Various attempts have been made to incorporate gravity, including Grand Unified Theories (GI'l) and Supersymmetric Theories (SUSY), but none of these theories have been confirmed experimentally.[3]

The Standard Model contains at least 21 different particles. There are 12 fermions: 6 leptons, $\left(e, \nu_{e}\right),\left(\mu, \nu_{\mu}\right)$ and $\left(\tau, \nu_{\mathrm{r}}\right)$ and 6 quarks, $(u, d),(c, s)$ and $(\ell$, b). There are also 12 bosons, 8 gluons $(g)$ which carry the strong force, the photon $(\gamma)$ which mediates the electromagnetic force, the $W^{ \pm}$and $\mathrm{Z}$ which carry the weak force, plus the Higgs boson $(H)$. Renormalizable gauge theories like the Standard Model allow for the presence of spin 0 particles. The usual formulation of the Standard Model recuires at least one spin 0 particle. The Iliggs boson is this spin 0 particle, which arises from the spontaneous symmetry breaking of $S U(2) \times U(1)$ and gives mass to the weak gauge bosons and fermions. All the fermions mentioned aloove have been observed except the $t$-quark (note, the $\nu_{r}$ has been inferred front $\tau$ decay). Or the 12 Standard Model bosons, all have been observed except the lliggs boson. The discovery of the lliggs boson would be great success for the Standard Model.[4]

The search for ever smaller particles means probing ever sinaller distance scales. In order to study these distances, wavelengths that are comparable to the scale being probed must be used. This implies higher and higher energies are needed in order to probe smaller and smaller distances. Accelerators, which provide beams of high energy particles, are used to probe these distance scales. One of the reasons for building the Superconducting Super Collider (SSC) in Texas is to search these small distance scales for the Iliggs boson and help confirm the Standard Model.[5]

\section{Experimental Status}

Most matter appears to be composed of quarks and leptons. Interactions among these particles, except for the gravitational interactions, are described by the Standard Model. Yet the Standard Model has over twenty free parameters[5] and this large number of parameters makes it hard to believe that this is a fundamental theory. The precise measurement of the $\mathrm{Z}$ niass can be used to define only one of these parameters. The mass of the lliggs boson and fermions, and the elements of the Kobayashi-Maskawa (KM) mixing matrix are some of the other parameters in the Standard Model.

In August of 1989, papers from both CDF and MARK 11 (an $e^{+} e^{-}$experiment at the Stanford Linear Accelerator Center) appeared in Physical Review Letters $\$ 6$, 7]; these papers presented the first precision measurements of the $Z$ mass and width. In July of 1989, LEP stored it's first beam and already experiments at LEP have improved the precision of the measurement of both the $Z$ mass and width. $[8,9$, $10,11]$ The inasses of the $t$-quark and the Higgs boson are also parameters of the Standard Model that can be measured experimentally, and direct evidence for these two particles has yet to be found. Preliminary measurements, made at CDF, place the inass of the $t$-quark above $89 \mathrm{GeV} .[12,13,14]$ The discovery of the Higgs boson is a major goal of particle pliysics. Present limits on the mass of the Higgs boson 
place the $\| I^{0}$ mass $>3.9 \mathrm{GeV}$ and the $I^{ \pm}$mass $>19 \mathrm{GeV}$.[3] The neutral lliggs $\left(H^{0}\right)$ is lowsed on the minimal Standard Model and the charged Iliggs $\left(H^{ \pm}\right)$is based on supersymmetric extensions to the Standard Model. The successes of the Standard Model suggest that these particles should exist; however, if they don't, the Standard Model will be on very shaky ground.

$\mathrm{CP}$-violation has been observed weakly in $K^{0}$-decays.[15] However, the magnitude of this violation is small and has only been observed through very precise measurements of the $K^{0} \vec{K}^{0}$-system. The origin of CP-violation (or T-violation) is a question the Standard Model doesn't address. [16] General principles of relativistic field theories, like the Standard Model, require CPT-invariance. If there is CP'Tinvariance, then CP-violation and T-violation are permitted. The KM-matrix can easily accommodate CP.violation through the inclusion of a complex phase factor. If this phase factor is non-zero, then CP-violation is allowed[17], lowever, this doesn't explain the origin of CP-violation.

Searclics for free quarks have been undertaken ever since the advent of the GellMann \& Zweig Qıark Model in the mid.60's. Yet all experiments since 1977 have presented negative results. $[3,18]$ This has led to the postulate of quark confinenent, implying that quarks are trapped inside hadrons. This idea of quark confinement is a key ingredient of QCD. Essentially, in QCD, quarks carry color, and experiments suggest only colorless particles can be observed. Questions of why quarks are confined and why no free quarks can be observed are two more questions the Standard Model doesn't address.
Qharks and leptons in the Standard Model can be grouped into generations, much like the Periodic Table of Elements can be grouped into rows and columns. There are currently three known generations or families in the Standard Model. 'These families are listed in Table 1. The first generation contains the $e, \nu_{e}$ leptons and the $u, d$ quarks. The next two generations are just heavier versions of the first generation.[17] Why is there more than one generation? This is one of the major unanswered questions of the Standard Model. Ilow many generations are there? Are there more than thrce? If there are not more than three generations, then why are there three? These are similar questions that may be asked. The number of light neutrino species can be found by measuring the width of the mass distribution for the $Z$ boson. This is because cach species of neutrino with mass less than one-half the $\mathrm{Z}$ boson mass contributes $180 \mathrm{MeV}$ to the width of the $\mathrm{Z}$ boson via the decay mode $\mathrm{Z} \rightarrow \nu \bar{\nu}$ and hence; the width of the $\mathrm{Z}$ boson mass distribution can be used to estimate the nunber of quark and lepton generations if all neutrinos are less than one-half the mass of the $\mathrm{Z}$ boson. Results from the four LEP experiments, at CERN, have ruled out a fourth light neutrino generation at the $98 \%$ confidence level. $[8,9$, 10, 11]

The large number of quarks and leptons has led to speculations that they are composite structures.[19] Most tests of QCD have only been comparisons to leadingorder calculations, next-lo-leading-order QCD calculations are now available. $[20,21$, 22) These new calculations should allow experimentalists to more precisely test QCD. These precision tests will either be able to verify predictions of QCD and the Standard 
Table I. A list of the Standard Model quark and lepton families and their properties.

\begin{tabular}{ccccc}
\hline \hline & \multicolumn{5}{c}{ Particle } \\
& Name & Symbol (type) & Clarge & Mass (GeV) \\
\hline \multirow{4}{*}{ First } & up & $u$ (quark) & $\frac{2}{3}$ & $4 \times 10^{-3}$ \\
Generation & down & $d$ (quark) & $-\frac{1}{3}$ & $7 \times 10^{-3}$ \\
& electron-neutrino & $\nu_{e}$ (lepton) & 0 & $<1.8 \times 10^{-8}$ \\
& $e$ (lepton) & -1 & $5.1 \times 10^{-4}$ \\
\hline \multirow{4}{*}{ Second } & charm & $c$ (quark) & $\frac{2}{3}$ & $1.5 \times 10^{0}$ \\
Generation & strange & $s$ (quark) & $-\frac{1}{3}$ & $0.2 \times 10^{0}$ \\
& muon-neutrino & $\nu_{\mu}$ (lepton) & 0 & $<2.5 \times 10^{-4}$ \\
& muon & $\mu$ (lepton) & -1 & $1.06 \times 10^{-1}$ \\
\hline \multirow{4}{*}{ Third } & truth & $t$ (quark) & $\frac{2}{3}$ & unknown \\
Generation & beauty & $b$ (quark) & $-\frac{1}{3}$ & $5 \times 10^{0}$ \\
& tau-neutrino & $\nu_{r}$ (lepton) & 0 & $<7 \times 10^{-2}$ \\
\hline \hline & tau & $\tau$ (lepton) & -1 & $1.78 \times 10^{0}$ \\
\hline \hline
\end{tabular}

Model, or show deviations from the predicted results. If QCD passes these tests, then all these unanswered questions will remain unanswered; however if deviations from QCD and experiment exist, then the question arises, is there something more? If there is an underlying structure to quarks and/or leptons, then history has shown that theories can usually be simplified when underlying structure is found.

Experiments at both CERN and Fermilab have measured the inclusive jet cross section. $[23,24,25,26]$ The higher energies available at the Fermilab Collider allows for a deeper search into the structure of quarks for compositeness. The current lower limit for the composite scale of quarks involving a contact interaction $\left(\Lambda_{c}\right)$, is $\Lambda_{c}>700 \mathrm{GeV}$.[26] This current litnit is from data collected by CDF in 1987 and is bascd on $\sim 26 n b^{-1}$ at $\sqrt{s}=1.8 \mathrm{TeV}$. The 1988-1989 $\mathrm{CDF}$ run collected more than 100 times this integrated luminosity at the same energy and should be capable of setting a limit of $\Lambda_{c}>1 \mathrm{TeV}$. The Standard Model successes indicate that if fermions are composite structures, this would happen on a size scale of less than $\sim 10^{-17} \mathrm{~cm}$ which inplies an composite scale of $\Lambda_{c} \geq 1 \mathrm{TeV}$.[5]

\section{Theoretical Motivation}

The Standard Model is the best theory available. Yet, as outlined above, there are still many unanswered questions such as: why are there three generations? The large number of fermions naturally leads to the question, are fermions elementary or is there something more? By measuring the inclusive jet cross section, the results of this measurement can be compared to QCD and check that the Standard Model is still valid. In the 1988-1989 CDF data run, CDF collected $\sim 4.2 p b^{-1}$ of data at $\sqrt{s}=1800 \mathrm{GeV}$. A measurement of the inclusive jet crose section from this data set should allow a search for the composite structure of quarks in the TeV range.

1. Quantum Chromodynamics

Jet are believed to originate from the scattering of the quarks $(q)$ and gluons $(g)$ that constitute hadrons. [21] The measurement of the jet cross section for the inclusive production of jets (inclusive jet cross section) is a basic measurement of the scattering from the interactions of these elementary particles. This measurement is therefore a quantitative test of the strong interaction theory $(\mathrm{QCD})$. 
Using the parton model ideas as discussed by E. Eichten et al. in Supercollider Physics [27], the leading order $\left(\alpha_{s}^{2}\right) \mathrm{QCU}$ processcs are all 2 body to 2 body processcs. Figure 1 is an example of a two jet event which illustrates what the result of a 2 2 body QCD process would look like in the CDF detector. These elementary 2-2 body subprocesses $\left(\hat{\sigma}_{i j}\right)$, listed in Table Il, are combined with the parton probability distributions $\left(f_{i}^{(a)}\left(x_{a}, Q^{2}\right)\right)$, to calculate the leading order $Q C D$ inclusive jel cross section. Equation 1 displays the form of the leading order QCD jet cross section used in this analysis and described by E. Eichten et al. $[27]$.

$$
\begin{aligned}
\frac{d \sigma}{d P_{1}} & =\int d y_{1} \int d y_{2}\left(\frac{2 \pi \tau P_{1}}{\hat{s}} \sum_{i,} \mid f_{i}^{(a)}\left(x_{a}, Q^{2}\right) f_{j}^{(b)}\left(x_{b}, Q^{2}\right) \hat{\sigma}_{i, j}(\hat{s}, \hat{i}, \hat{u})\right. \\
& \left.\left.+f_{j}^{(a)}\left(x_{a}, Q^{2}\right) f_{i}^{(b)}\left(x_{b}, Q^{2}\right) \dot{\sigma}_{i, j}(\hat{s}, \dot{u}, \hat{i})\right] /\left(1+\delta_{i},\right)\right\}
\end{aligned}
$$

where $y_{1}$ and $y_{2}$ represent the rapidity of jets 1 and 2 respectively, $P_{l}$ is the common transverse momentum of the two jets, $\dot{s}=s \tau$ is the square of the parton-parton subenergy,

$$
\begin{aligned}
\tau & =\frac{4 P_{t}^{2}}{s} \cosh ^{2} \frac{y_{1}-y_{2}}{2}, \\
x_{a} & =\sqrt{\tau} e^{\frac{y_{1}+y_{2}}{2},} \\
x_{b} & =\sqrt{\tau} e^{-\frac{y_{1}+y_{2}}{2},} \\
\hat{t} & =-\frac{\hat{s}}{2}(1-\cos \theta), \\
\hat{u} & =-\frac{j}{2}(1+\cos \theta)
\end{aligned}
$$

\section{and $Q^{2}$ is the appropriate scale of the hard scattring process.}

The choice of $Q^{2}$ in Equation $I$ is ambiguous since any shift in $Q^{2}$ introdnces terms in $\sigma_{i j}$ of order $\alpha_{s}^{3}$ which are neglected in order $\alpha_{s}^{2}$ calculations. Ilowever,

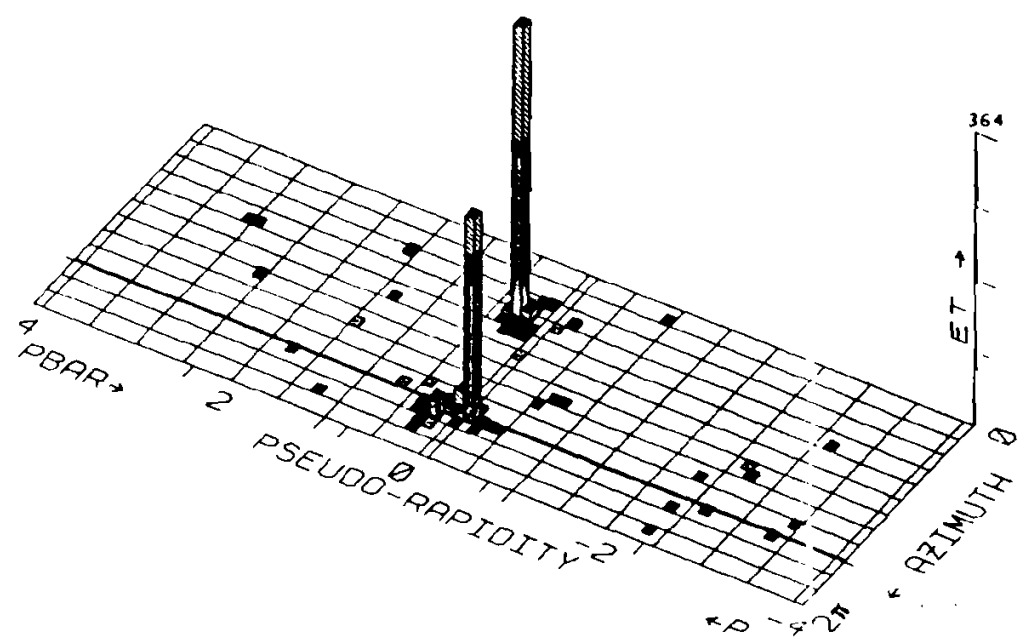

Figure 1. An example of a two jet event, in the CDF detector, which illustrates a 2-2 body QCD process. In $\eta$ - $\phi$ space this figure illustrates the location of clusters of energy, jets, in the CDF detector.

according to QCD perturbation theory, the choice of $Q^{2}$ should be characteristic of the scale of the hard scattering process. Therefore, $Q^{2}$ was chosen to be equal to $P_{1}^{2} / 2$ in all the leading order QCD calculations used in this analysis and $P_{t}^{2} / 4$ for the next-to-leading order QCD calculations.

Detailed quantitative studies of the inclusive jet cross section are limited by the ambiguities in the calculation of the cross section. The theoretical ambiguitieg include the uncertainty in the choice of $Q^{2}$ as mentioned above and the uncertainties in the parton distribution functions. Both theoretically and experimentally the precise definition of a jet is also ambiguous. Different experiments are free to define jets in slightly different ways, theoretically at order $\alpha^{2}$ the jet that is measured in the detector 
Table 11. Elementary cross sections for leading order $\alpha_{s}^{2}$ QCD subprocesses, given in the form $(\hat{s} / \pi) d \hat{\sigma} / d \hat{t}$.

\begin{tabular}{|c|c|}
\hline ubp & Elem \\
\hline$q_{i} q_{j} \rightarrow q_{1} q_{j}$ & \\
\hline$q_{1} \bar{q}_{j} \rightarrow q_{i} \bar{q}_{j}$ & \\
\hline$q_{i} \bar{q}_{i} \rightarrow q_{j} \bar{q}_{j}$ & \\
\hline$q_{i} q_{i} \rightarrow q_{i} q_{i}$ & $\dot{\underline{i}}^{2}-\frac{2 j}{3 \dot{u}}$ \\
\hline$q_{i} \bar{q}_{i} \rightarrow q_{i} \bar{q}_{i}$ & $\left.\frac{\dot{u}^{2}}{2}-\frac{2 \dot{u}^{2}}{3 \vec{i} i}\right]$ \\
\hline$q_{i} \bar{q}_{i}-$ & \\
\hline$g g \rightarrow q_{i} \bar{q}_{i}$ & \\
\hline$g q_{i} \rightarrow g q_{i}$ & \\
\hline$g g \rightarrow g g$ & $\left.-\frac{j i}{\hat{u}^{2}}\right]$ \\
\hline
\end{tabular}

is associated with the parton, and therefore the jet properties are corrected in order to associate the varions quantities with the corresponding partonic quantities. This assumes the parton associated with each jet is detected as a narrow jet of particles

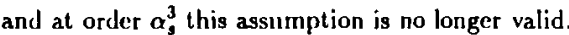

The 2-3 body parton scattering subprocesses are listed in Table III. Figure 2 is an example of a three jet event in the CDF detector which illustrates a $2-3$ body QCD process. The matrix elements squared for all $2 \rightleftharpoons 2$ and $2-3$ parton scattering subprocesses have been calculated.[28] An immediate consequence of this fact is the ability to calculate the next-to-leading order QCD inclusive jet cross section. [20, $21,22]$ At this level a jet can consist of more than a single parton, and therefore the definition of a jet at the theoretical and experimental level become intertwined.
'Table III. List of next-to-leading order $\alpha_{0}^{3}$ QCD subprocesses.

$$
\begin{aligned}
& \text { Subprocess } \\
& \hline q_{1} q_{j} \rightarrow q_{i} q_{j} g \\
& q_{1} g \rightarrow q_{i} q_{j} \bar{q}_{j} \\
& q_{i} \bar{q}_{j} \rightarrow q_{i} \bar{q}_{j} g \\
& q_{i} \bar{q}_{i} \rightarrow q_{j} \bar{q}_{,} g \\
& q_{1} q_{i} \rightarrow q_{i} q_{i} g \\
& q_{i} g \rightarrow q_{i} q_{i} \bar{q}_{i} \\
& q_{1} \bar{q}_{i} \rightarrow q_{i} \bar{q}_{i} g \\
& q_{1} \bar{q}_{i} \rightarrow g g g \\
& q_{i} g \rightarrow q_{i} g g \\
& g g \rightarrow q_{i} \bar{q}_{i} g \\
& g g \rightarrow g g g \\
& \hline \hline
\end{aligned}
$$

This is because a decision must be made as to when to count two partons as one jet and when to count them as two jets. In the next-to-leading order calculation of the inclusive jet cross section, the jet definition was chosen in order to agree with the experimental definition as much as possible, thus eliminating possible ambiguities associated with this definition.[21]

A prediction that arises from the definition of the jet in these calculations is the dependence of the cross section on the jet definition. A fixed cone algorithm was used both thcorctically and experimentally to define the jet; the clustcring cone size was an adjustable parameter in this definition. The inclusive jet cross section at order $\alpha_{0}^{3}$ is dependent on the cone size used in measuring these jets. The dependence of the 


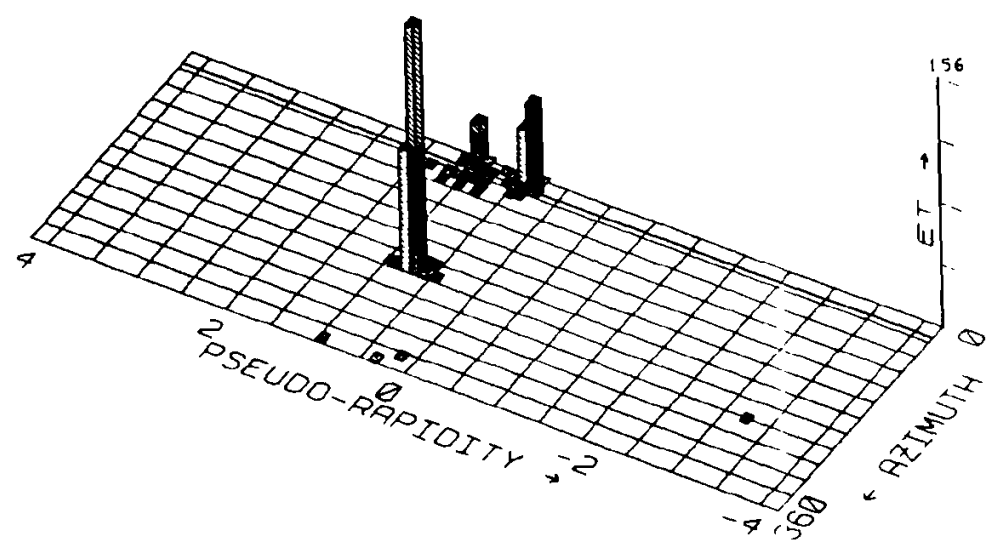

Figure 2. An example of a three jet event, in the CDF detector, which illustrates a 2-3 body QCD process. In $\eta-\phi$ space this figure illustrates the location of clusters of energy, jets, in the CDF detector.

cross section on the cone size is an intrinsic feature of QCD and it is important that this be tested experimentally.[21] Due to the fact that the jet is no longer associated with a parton, the jet and its properties as measured in the detector are no longer corrected back to the parton level. Jet corrections must only account for detector effects and not theoretical effects. In this analysis, this implies that corrections for energy lost outside the clustering cone associated with the parton should no longer be applied.

\section{Compositeness}

In the search for possible breakdown in the Standard Model, it is essential to explore the scattering of the elementary constituents of matter at the highest $E_{i}$ 's possible. The composite structure of quarks and gluons is one possible source of breakdown in the Standard Model. The measurement of the inclusive jet cross section using the CDF detector allows compositeness to be scarched for at the highest $E_{t}$ 's currently available in the world.

The basic assumption underlying nost composite models is that the constituent particles interact by means of a new strong gauge interaction.[27] This new strong interaction binds the constituent particles into singlet states at energies below some characteristic scalc $\Lambda_{c}$. At encrgies of a few times the composite scale $\Lambda_{c}$, multiple production processes should dominate over the familiar 2-2 body parton scattering processes. Which processes will occur is model dependent, but in general, the cross section for allowed inelastic processes will be of order $4 \pi / \Lambda_{c}$ and will completely dominate over the standard processes for which the cross section is of order $\pi a_{i}^{2} / \mathbf{s}$ $\Lambda t$ energies less than $\Lambda_{c}$ the comparison with the Standard Model is quantitative.

Compositeness (quark structure) can be approximated by the effect of a contact term added to the QCD lagrangian. In $p \bar{p}$ collisions the production of high-transverse momentum jets is most sensitivc to the presence of a flavor-diagonal contact term $L_{q q}$

$$
L_{q q}=\frac{ \pm g^{2}}{2 \Lambda_{c}} \bar{q}_{L} \gamma^{\mu} q_{L} \bar{q}_{L} \gamma_{\mu} q_{L}
$$

is the form of the contact term for the coupling of left-handed quarks, there are, however, other possible terms. The contact term modifies the cross section and the effect are most apparent at the large valucs of $P_{t}$ for which the valence quark interactions dominate the jet cross scction 
Comparisons of the measured inclusive jet cross section to composite model predictions based on the presence of a fiavor-diagonal contact term will be Inade in this analysis. By normalizing, the theoretical predictions to the data at sunall values of $E_{t}$ where the composite effects are not significant, then the cross section at the higher values of $E_{\mathrm{l}}$ can bc examined in order to scarch for a possible composite signal. The following chapters will examine how this was accomplished using the most recent data collected by CDF. 


\section{CHAPTER II}

\section{THE CDF DETECTOR}

The Collider Detector at Fermilab (CDF) is a general purpose spectrometer designed to explore the details of physics processes at the Fermilab Tevatron center of mass encrgy, $\sqrt{s}=1.8 \mathrm{TeV}$. In order to measure and identify particles produced at the Tevatron Collider, clarged particle tracking, magnetic momentum analysis and fine grained calorimetry are combined to measure the energy, momentum, and where possible, identify the particles. The detector has three main components as shown

in Figure 3. These components are a movable central detector and two identical forward/backward detectors situated on either side of the central detector. Figure 4 shows a cut-away view of one half of the complete CDF detector system, the detector is forward/backward symmetric about the interaction point.

- The coordinate system for CDF is right-handed and defined to have the origin in the center of the central detector. The $x$-axis points radially outward in the plane of the Tevatron (the Tevatron is a circular colliding beam machine) forning a line that intersects both the center of the CDF detector and the center of the Tevation. The $y$-axis is perpendicular to the $x$-axis and points upward from the center of the detector. The 2-axis points in the direction that the protons are transported, langent to the Tevatron, originating at the center of the central detector. The radius ( $R$ ), azimuthal angle $(\phi)$, polar angle $(\theta)$ and $z$ coordinate in the detector system arc defined according to standard definitions using this coordinate system.

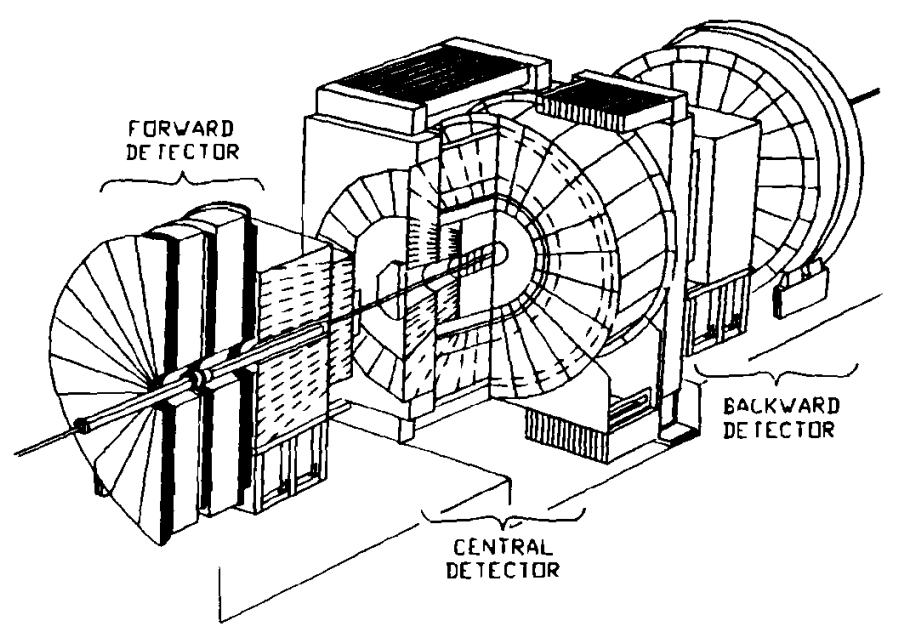

Figure 3. Perspective view of the CDF detector showing the central detector and the forward/backward detectors.

The central detector consists of a solenoid magnet, tracking chambers, electromagnetic shower counters, hadronic calorimeters and muon chambers. The forward/backward detectors consist of segmented time-of-flight counters, electromagnetic shower counters, hadronic calorimeters, and muon toroidal spectrometers. The individual detector components for the central and forward/backward detector systems will be described briefly here. The detector is described in detail elsewhere.[29] Particular attention will be given to the various individual detector systems used in this analysis. 


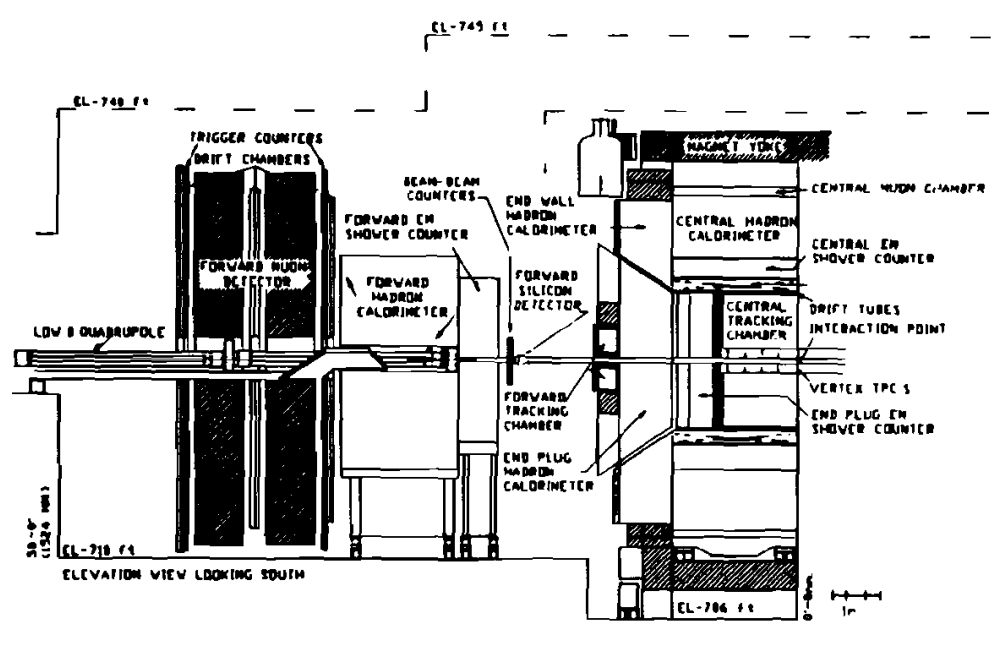

Figure 4. Cut-away view of one-half of the CDF detector showing the individual detector sub-systems making up the central and forward/backward detec. tors.

\section{A. Tracking}

The tracking system used in conjunction with the magnetic field supplied from the solenoid is used to reconstruct particle trajectories and momenta. Particles scattered in the transverse plane of the beam, which originate from proton-antiproton $(p \bar{p})$ collisions near the center of the detector, can be observed in the tracking chambers that surround the region. The vertex time projection chamber (VTPC) is used to find the position of the initial collision and track the charged particles produced at polar angles of greater than 3.5 degrees. The forward/backward tracking chamber (FTC) can reconstruct particles produced in the angular region from 2-10 degrees/170178 degrees and the central tracking chamber (CTC) combined with the central drift tubes (CDT) provides a correlated R- $\phi-z$ measurement of the charged particle tracks in the region from 15165 degrees.

The data from the VTPC supplied infornation about the $\mathrm{z}$ position of the vertex for the event, which was used to obtain the corrected jet pseudorapidity ( $\eta$, where $\left.\eta=-\ln \left(\tan \left(\frac{\theta}{2}\right)\right)\right)$ and reject jet backgrounds (see Clapter 111$)$. Tracking information in the central detector region was used to measure the jet fragmentation propertics (see (liapter IV) and to calculate the charged hadron fraction used in rejecting jet backgrounds (sce Chapter III).

I. Vertex Time P'rojection Chamber

The VTPC covers $\sim 7$ units of $\eta$ and was optinized to have good pattern recognition in R-z in order to locate the event vertex and to compliment the R- $\phi$ tracking of the CTC.[30] The VTPC was designed to find the $\mathrm{z}$ vertex of the event, determine the overall event topology, perform charged particle measurements for polat angles of 3.5176 .5 degrces, identify multiple interactions, provide intermediate tracking in the angular region of 10-30 degrees and forward tracking from 3.5-10 degrees. This was required to be done in such a way as to minimize the effects of secondary interactions and multiple scattering, which can degrade electron identification and adversely affect the CTC momentum resolution.

'The V'Tl'C consists of eight time projection chambers (TPC) surrounding the bcam pipe nounted end to end along the beam direction (2-axis). Each of the eight octagonal modulcs has a central high voltage grid that divides the module into two 
$15.25 \mathrm{~cm}$ long drift regions. Electrons drift away from this grid and enter the proportional chramber endcaps. The endcaps are divided into octants, where each octant contains 24 sense wires and 24 cathode pads.

The arrival times of the electrons provide the detector information on the $\mathrm{R} \cdot \mathrm{z}$ shape of the event. Flash analog to digital convertors (FADC's) comnected to the sense wires and pads in some endcaps provide $\mathrm{dE} / \mathrm{dX}$ and $\phi$ information for particles with a polar angle of 5-25 degrees. Adjacent modules have a relative rotation angle of $\phi_{0}=\arctan (0.2)$ about the beam axis, this eliminates inefficiency for particles transversing 2 modules near octant boundaries and provides $\phi$ information from a small angle stereo measurement.

The resulting performance for the VTPC is described in the following way. For tracks at 90 degrees, the $\mathrm{z}$ resolution is $420 \mu \mathrm{m}$ and at 11 degrees the $\mathrm{z}$ resolution is $1100 \mu \mathrm{m}$. The impact parameter (b) for tracks with a primary vertex was found to have a rms of $0.3 \mathrm{~cm}$ and the overall charuber precision in $\phi$ is on the order of $100 \mu \mathrm{m}$. The 2-track resolution in $z$ is a function of the polar angle, in $\mathbb{R}$ the resolution is $6 \mathrm{~mm}$ and in $\phi$ the resolution is $3 \mathrm{~cm}$.

2. Central Tracking Chamber

The CTC was designed for operation in the high magnetic fields and the high density track environment of hadron colliders. It was designed to compliment the calorimeters by providing single particle infornation. The most important feature of the CTC is its ability to measure high transverse momentum $\left(P_{t}\right)$ isolated tracks which are critical to measurements of high $P_{l}$ leptons.[31]
The CTC is a large cylindrical drift chanber consisting of 84 layers of sense wires arranged in 9 superlayers. Five superlayers have the sense wires arranged parallel to the beam axis, earh superlayer containing 12 sense wire layers. Four superlayers are sterco layers which have their sense wires at an angle of \pm 3 degrees to the beam axis. There are 6 sense wire layers in each stereo superlayer, and the four stereo superlayers are interweaved with the five axial superlayers. The superlayers are divided into cells such that the maxinum drift distance is less than $40 \mathrm{~mm}$ ( $800 \mathrm{~ns}$ drift time). The chamber surrounds the VTPC and has an inner radial distance of $277 \mathrm{~mm}$, an outer radial distance of $1380 \mathrm{~mm}$, and a length of $3201.3 \mathrm{~mm}$.

The CTC was found to have a 2 -track resolution of $3.5 \mathrm{~mm}$, and a spatial resolution of less than $200 \mu \mathrm{m}$ in R- $\phi$ and less than $6 \mathrm{~mm}$ in $z$. The momentum resolution of the detector is coupled to the VTPC and the polar angle of the tracks relative to either the proton or antiproton direction. In the angular region of 40 140 degrecs, the momentum resolution is better than $\delta p_{T} / p_{T}^{2} \leq 0.002(\mathrm{GeV} / c)^{-1}$.

3. Central Drift Tubes

The CIDT was designed to provide high accuracy $\mathrm{R}-\phi-z$ information at a radius of $1.4 \mathrm{~m}$ for charged particles in the central region of CDF. Operating in the limited streaner mode (in order to maximize the $z$ resolution), charge division along anode wires provides R-z information, and drift time measurements in the three layers on the CDT array provides R- $\phi$ information.

Located on the outer surface of the CTC and inside the solenoid, the CDT consists of 2016, $12.7 \mathrm{~mm}$ diameter tubes, $3 \mathrm{~m}$ in length arranged in three layers of 672 tubes each. [32] The information from the CDT was used in conjunction with the 
VTPC and CTC to provide charged tracking information in the $\eta$ range of \pm 1 . The CDT has a typical resolution of $2.5 \mathrm{~mm}$ in $\mathrm{z}$ and $200 \mu \mathrm{m}$ in $\phi$.

\section{Forward Tracking Chamber}

The FTC is a radial drift chamber designed to measure and track charged particles in the polar angle range of 2-10 degrees and 170-178 degrees, while operating in a high rate, high track multiplicity environment. It was capable of multi-track resolution of $2-3 \mathrm{~mm}$ and had tracking accuracy of $140 \mu \mathrm{m}$ per wire.[33]

The chambers consisted of 72 wedge shaped cells with planes of anode and field shaping wires that alternate with cathode strips. The wire and cathode planes of each 5 degree cell are slanted at 2 degrees relative to the beam axis so left right ambiguities can be resolved. The anode plane as 21 active $50 \mu \mathrm{m}$ diameter sense wires and 26 field shaping wires $150 \mu \mathrm{m}$ in diameter strung normal to the beam axis. The drift space covered from $5.4 \mathrm{~mm}$ at the inner radius to $28.2 \mathrm{~mm}$ at the outer radius. A total of 3024 active sense wires in both the forward and backward directions provide a R. $\phi-z$ measurement for each track.

\section{B. Solenoid Magnet Coil}

The precise momentum for each cliarged particle in the CTC was determined using the CTC information while operating in a uniform $1.4 \mathrm{~T}$ magnetic field oriented along the beam direction. This field was provided by a $3 \mathrm{~m}$ diameter $5 \mathrm{~m}$ long superconducting solenoidal coil. The coil was made of 1164 turns of aluninum stabilized $\mathrm{NbTi} / \mathrm{Cu}$ superconductor, with an overall radiation thickness of 0.85 radiation lengths.[20]

\section{Calorimeters}

The calorimeters at CDF use a tower geometry because of the importance of jets in ligh energy $p \bar{p}$ collisions. 'This type of geometry allows jets to be easily identified. The (D)F calorimetry spans an $\eta$ range of \pm 4.2 and has full azimuthal coverage. The calorimeters arè segmented by 0.1 in $\eta$ and 15 degrees in $\phi$ for the central and 5 degrees in $\phi$ for the forward and plug regions. All calorimeter towers have both electromagnetic and hadronic segmentation to allow a detailed comparison of the electromagnetic and hadronic energy composition in the events. In the central region the electromagnetic calorimeters consists of alternating layers of lead absorber and an active medium of scintillator, and in the plug and forward regions the active medium are proportional tubes with pad readout. The hadronic calorimeters consist of the saıne active mediums, but use steel for the absorber.

In this analysis the central electromagnetic and hadronic calorimeters (CEM and CllA) were used to measure the properties of the jets used in the cross section. Jets measured in the plug electromagnetic and hadronic calorimeters (PEM and PHA) and forward electromagnetic and hadronic calorimeters (FEM and FIIA) were used to reject background in the events.

1. Central Electromagnetic Calorimeter

The CEM was designed to incorporate good energy resolution with fine segmentation. This was accomplished by using scintillator and wavelength shifter to measure the energy and an embedded strip chamber for a position determination and longitudinal shower development. The average energy resolution, $\sigma(E) / E$, of $13.5 \% / \sqrt{E \sin (\theta)}$ and a position resolution of $\pm 2 \mathrm{~mm}$ at $50 \mathrm{GeV}$ was measured. [34] 
The CEM consists of 48 modules each of length 98 in spanning 15 degrees in azimuthal angle. Four arches of 12 modules each surround the central interaction region on the outside of the solenoid. A total of 31 layers of $5 \mathrm{~mm}$ thick SCSN-38 polystyrene scintillator wrapped in 2 layers of 0.0015 in vellum drawing paper were assembled to form 10 projective towers. Interleaved with the scintillator are 30 layers of $1 / 8$ in lead clad with 0.015 in of aluminum. The strip chamber was inserted between the $8^{\text {th }}$ and $9^{\text {th }}$ layers of the scintillator, at a depth corresponding to the maxirnum average transverse shower developinent. One wedge was notched with a gap to allow cryogenic service access to the solenoid.

2. Hadronic Central and Endwall Calorimeter

Covering the full $2 \pi$ azimuthal range and a polar angle from 30-150 degrees, the CHA and endwall hadronic (WHA) calorimeters consist of 48 stcel scintillator modulcs each. Designed to have good energy resolution and uniformity, the calorimeters were initially designed to have an energy resolution of $\sigma\left(E^{\prime}\right) / E=0.50 / \sqrt{E}$. Due to other constraints, the total thickness of $80 \mathrm{~cm}$ of steel at normal incidence resulted in only $95 \%$ containment at $50 \mathrm{GeV}$, therefore the energy resolution degrades at high energy and muon identification is more difficult.[35]

In the central calorimeter the sampling length is $2.5 \mathrm{~cm}$; in the endwall the sampling is $5.0 \mathrm{~cm}$. Each module is divided into projective towers as in the CFM. This segmentation is fine enough that the quark and gluon jets will nornally spread over more than one tower. For each 15 degree slice there are 24 towers, 12 completely in the central, 6 completely in the endwall, and 6 shared between the endwall and central region. The central region covers 45-135 drgrees in polar angle and is 32 layers deep. The endwall covers 30-45 degrees and 135-150 degrees in polar angle, serves as part of the flux return path for the solenoid, and is 15 layers deep. The active medium is $1 \mathrm{~cm}$ thick PMMA scintillating plastic doped with $8 \%$ naphthalene, $1 \%$ butyl-PBD and $0.01 \%$ POPOP. The light is collected by wavelength shifters mounted on the sides of the modules.

3. Plug Electronagnetic Calorimeter

The PEM is $2.8 \mathrm{~m}$ in diameter, $50 \mathrm{~cm}$ deep and uses a lead proportional tube sand wich combined with cathode pad and strip readout to measure energy in the plug regions of the CDF detector. Designed to close the solenoid volume at both ends of the central detector, it is required to operate in a $15 \mathrm{kG}$ field, have fine segmentation, good hermeticity, and good energy resolution.[36] This fine segmentation was essential for isolating clectrons and identifying jets.

The PEM covers both ends of the $3 \mathrm{~m}$ diameter $5 \mathrm{~m}$ long solenoid and occupies a cylindrical hole with an outer radius of $280 \mathrm{~cm}$ and a depth of $53 \mathrm{~cm}$, covering a polar angle of 10-36 degrees with full depth coverage up to 32 degrees. It consists of 4 quadrants on each end, each quadrant consisting of 34 layers of proportional chambers interwoven with $2.7 \mathrm{~mm}$ lead. Pads are etched on one side of the proportional chambers to form a projective readout geometry. The layers are grouped into three depths. The first and last depth consist of 5 layers, the second depth uses 24 layers. Strips are etched on the other side of the chambers in layers 6-15, these strips alternate between two orthogonal directions layers $6,8,10,12$, and 14 have strips in the azimuthal direction and layers $7,9,11,13$, and 15 have strips in the radial direction. 
4. Plug Hadronic Calorineter

Tlu: l'ilA has an angular coverage from 10-30 degrees and 150-170 degrees in polar angle and complete azimuthal coverage. It is segmented into 12,30 degree sectors on either side of the interaction region. Each sector consists of 20 proportional chambers with cathode pad readout sandwiched between $21,5.1 \mathrm{~cm}$ thick steel plates.[37] Each proportional chamber consists of proportional tubes inade of 50 micron diameter gold plated tungsten wire centered in a resistive plastic tube, sandwiched by a copper ground plane and copper cathode plane. Each clamber consists of 72 pads arranged to form projective towers in $\eta$ and $\phi$.

5. Forward Electromagnetic Calorimeter

The FEM was designed to be radiation resistant and used in the small angle region in the CDF detector system. Each calorimeter consists of four quadrants containing 30 layers of proportional tube chambers witls cathode pad readout sandwiched in between lead sheets.[38] The pads are ganged longitudinally to form a two tower depth segmentation of 15 layers each. There are a total of 1440 pads per layer for a total $\mathbf{5 7 6 0}$ tower segments for both the forward and backward calorineters. Anode wires are strung vertically and are ganged into 5 sectors to provide information on the longitudinal development of the events

6. Forward lladronic Calorimeter

Texas A\&M University was responsible for the design and construction of the Forward/Backward Hadron Calorimeter (FHA) for the CoF Detector. The FllA was designed to detect and measure the energies and positions of hadrons in the pseudorapidity range of $2.2 \leq|\eta| \leq 4.2$ with full azimuthal coverage with respect to the b..... . Ixis.[39] The FIIA consists of both a forward and a backward calorimeter; each calorimeter is divided into four quadrants. The quadrants are made of $27(204 \mathrm{~cm}$ $\times 196 \mathrm{~cm} \times 2.5 \mathrm{~cm})$ gas proportional tube chambers sandwiched in between 27 $(213 \mathrm{~cm} \times 213 \mathrm{~cm} \times 5 \mathrm{~cm})$ steel plates. The chambers are constructed with a cathode pad segmented, in pscudorapidity $(\Delta \eta=0.1)$ and in azimuth $(\Delta \phi=5$ degrees), into smaller pads. Signals from each of the smaller pads $(\Delta \eta=0.1, \Delta \phi=5$ degrees $)$ at fixed $\eta$ and $\phi$ are summed and form a projective tower in $\eta$ and $\phi$, pointing back to the interaction region of the CDF Detector. Each chamber also contains anode wires segmented into six regions which can be read out separately or summed, thus allowing the measurement of a longitudinal shower distribution for each event.

D. Muon Detection

There are two detector systems to detect and ineasure muons at CDF. In the central region, cach calorimeter wedge contains four layers of muon chambers. In both the forward and backward directions there is a muon spectrometer consisting of magnctized steel toroids, drift chambers, and triggering scintillation counters. Although neither of these systems are used directly in this analysis, muon hits in the central region were used when scanning events to determine the quality of the jet events.

1. Central Muon Chambers

The central muon chambers (CMU) cover the central rapidity region, $|y| \leq \mathbf{0 . 7}$, in the CDF detertor. The chambers in the CMU were designed to detect, identify, 
and measure the direction and position of muons by the penetration of muons through 4.9 absouption lengths of material in the central calorimeter. $[40]$

The detector is located on the outside of the CHIA at a radial distance of $3470 \mathrm{~mm}$ from the beam axis and is segmented in $\phi$ into 12.6 degrec wedges. Earh detector is further segmented into 3 modules of 4.2 degrecs each in $\phi$. Each module consists of 4 layers of 4 rectangular drift cells. A stainless sted resistive sense wire is located in the center of each cell. A single timing measurement is used for the measurcments in the drift direction $(\phi)$, while charge division on the sense wire is used for measurements of $\eta$. The clamber operates in the limited streamer node, has a rms resolution of $250 \mu \mathrm{m}$ in the drift direction and a rms resolution of $1.2 \mathrm{~mm}$ along the sense wire direction.

2. Forward Muon Chambers

The forward muon spectrometers (FMU) consists of a pair of magnetized iron toroids instrumented with 3 sets of drift chambers and 2 layers of scintillation trigger counters.[41] It was designed to measure the position and moment um of muon in the polar region from 3-16 degrees and 164-177 degrees in the forward/backward region of CDF.

Each plane or set of detectors consists of 24 wedge shape chambers subtending a azimuthal $(\phi)$ angle of 15 degrees. Each wedge is staggered relative to its neightbors to form overlap regions and eliminate dead spots and detector boundarics. The turoids are $1 \mathrm{~m}$ deep, with an outcr diameter of $7 \mathrm{~m}$ and an inner diameter of $1 \mathrm{~m}$. Two of the four 395 ton magnets are located at both the forward and backward detector regions. Each toroid is made of 4 rectangular coils consisting of 28 turns of copper to provide an azimuthal field in the steel. Each chamber consists of two planes of drift cells. The plane closest to the interaction region consists of 56 cells and the other plane consists of $\mathbf{4 0}$ cells staggered relative to the first plane so as to resolve left-right ambignities. Each cell contains a stainless steel sense wire strung along the cord to provide a pular angle ineasurement and the two sides of the cliamber share a common copper foil cathode plane divided into 15 cathode pads.

F. Data Acquisition Systen

The CDF detector has a total of 100,000 electronic channels for readout of the various detector conponents. The calorimetry requires a large dynamic range to measure encrgy of a few tens of $\mathrm{MeV}$ to hundreds of $\mathrm{GeV}$; therefore a special front end electronic system called RABBiT was developed to handle these requirements. Belore any of this conld be readout, a trigger system also had to be developed to exploit the design of the projective geometry in the calorimeter towers. The beambeain counters arc the first layer of the trigger system. The various aspects of these systems will be discussed briefly in this section.

1. Front End Electronics

The front end electronics at CDF are based on the RABBIT (Redundant Analog Bus-Based Information Transfer) system.(42] This crate based front end system features low cost, compact packaging, fast readout, command capability, 16 bit digitization and a high degree of redundancy. The crates can contain a variety of instrunentation modules and are designed to be placed near the detector. Since access to the CDF detector is not possible during machine operation, the electronics had to 
be designed to perform all normal operations without physical access to the system. The components of the RABBI'T system are the RABBI'T crate, the system modules, front end instrumentation modules, and special purpose processors to direct readout. The overriding design specifications for the system required it be able to read nany calorimeter channels within 1-2 ms for which the ratio of full scale signal to calibration signal would be 1000:1. It was also necessary to have a calibration accuracy of $1 \%$.

2. Data Acquisition

All CDF events are collected in a multilevel FASTBUS network. [43] The choice of FASTBUS was motivated by the ability of FASTBUS to support high data rates and both high speed devices (synchronous transfers) and low speed devices in asynclironous full liandshake transfers. The data acquisition (DAQ) and trigger systems operate in a hierarchical structure. The level 1 and 2 triggers must filter the raw interaction rate down to $100 \mathrm{llz}$ or less. The $100 \mathrm{llz}$ rate a practical limit at which events can be digitized and bufferce.

If an event is accepted by both the level I and level 2 triggers, the front end crates are digitized and readout by scanner modules. The calorimetry and the central nuoul cliambers are readout by MX scanners (MX scanners are used to access RABBIT front end electronics), and the tracking systems use a SSP scanner (SSP scanners are used to access FASTBUS based front end electronics). Each scanner has four event buffers. Once all MX and SSP scanners have finished reading and buffering an event, a trigger supervisor module signals the buffer manager the cvent is ready. The buffer manager (BFM) controls data flow from the scanners to the VAX host computers. The BFM signals the event builder (EVB) to read a specified buffer and reformat the complete event. When the EVI3 finishes, it signals the BFM, which in turn iustructs the trigger supervisor the specified buffer is now available for a new event.

Fron the FVB, the event is passed onto the level 3 trigger which uses VME based processors and control nodules developed by the Fermilab Advanced Computer Program (ACP). The BFM instructs the EVB to write out an event to one of the ACP processors. Events accepted by level 3 are then read by the Buffer Multiplexor executing on the VAX cluster. The event can then be logged to disk or tape to be accessed in real time by computer processes executing on both host and remote VAX computers using DECNET.

3. Beam-Beam Counters

The beam-beam counters ( $\mathrm{BBC}$ ) provide a minimum bias trigger for the CDF detector and are used as the primary luminosity monitor for CDF.[29] The BBC consists of a plane of scintillation counters on the front face of each the forward and backward shower counters, each consisting of 16 time-of-Aight counters. These counters (BDC) have excellent timing properties (a timing resolution of $200 \mathrm{ps}$ ) and provide the best measurement of the time of the interaction, as well as a crude measureneit of the $z$ vertex (within $10 \mathrm{~cm}$ at the trigger level). These counters are arranged in a rectangle around the beam pipe and cover the angular region (measured in either the horizontal or vertical direction) of $0.32-4.47$ degrees. 


\section{Triggering}

$\Lambda$ two level FASTBUS based trigger system provide the initial triggers for the CDF detector.[44] The purpose behind this multi-level trigger system is to introduce as little bias as possible at the lowest trigger levels, while reducing the event rate so the higher trigger levels can do a more sophisticated analysis without a significant amount of time lost. The readout of the $\operatorname{CDF}$ detector by the data acquisition system is the most time consuming process which takes on the order of $1 \mathrm{lng}$.

Analog signals are used to form sums and moments before digitization in order to have a fast trigger. This is done using 10 FASTBUS analog crates which can be identified by type of calorimeter signal (electromagnetic or hadronic) and geographic location (central, plug and forward detector regions). Calorimeter towers are summed into towers of $\Delta \phi=15$ degrees and $\Delta \eta=0.2$, these signals are then sent to the corresponding FASTBUS analog crate. Receive and weight (RAW) cards in the crates compensate the various detector components so that a $50 \mathrm{GeV}$ transverse energy ( $E_{\ell}$, where $\left.E_{t}=E \sin (\theta)\right)$ deposition produces a -1 volt gignal. These signals are passed onto a compare and sum (CAS) card which sums all the towers in a single RAW card above some threshold. The CAS card sums three different quantities, the linear sum, the sum weighted by $\sin \phi$, and the sum weighted by $\cos \phi$. An inclusion register controls which channels will be added into the sum so that bad channels can be turned off.

The crate sum boards take the three sums $\left(E_{1}, E_{1} \sin \phi\right.$ and $\left.E_{t} \cos \phi\right)$ and converts them from analog to digital signals. At the same time, since each CAS card has a mean $\eta$ hardwired into it, the crate sum board also calculates the sum $E_{t} \eta$ and
$E_{\ell} \eta^{2}$. After being digitized by the crate sum, the sums for all the separate detector componcnts are brought together in the level I sum board. There is one level I sum board for each quantity summed $\left(E_{t}, E_{t} \sin \phi\right.$ and $\left.E_{t} \cos \phi\right)$. Each board creates a grand total sum of the electromagnetic, hadronic and combined $E_{l}$, which are then compared to programmable thresholds.

The level 2 trigger uses a cluster finder board interacting with the CAS board. In level 2, the outputs from the CAS boards are loaded into the cluster finder which then process the data and returns a set of towers corresponding to one cluster of energy. The algorithm for finding clusters used in the cluster finder is the nearest neighbor algoritlim and will be discussed in Chapter III. Following a similar path as in level 1, level 2 then proceeds to find the $E_{\imath}, E_{\mathfrak{l}} \sin \phi$, and $E_{l} \cos \phi$ for each individual cluster, as well as $E_{t} \eta$ and $E_{t} \eta^{2}$.

The first two leveis of the CDF trigger, as discussed above, are able to reduce the raw event rate of $50-75 \mathrm{kHz}$ to $1-100 \mathrm{~Hz}$ introducing as little bias as possible. 


\section{CHAPTER III}

\section{MEASUREMENT OF JETS AT CDF}

The processes required to measure the inclusive jet cross section are exarnined in this chapter. The measurement of the cross section begins with the definition of a jet and the physical quantities associated with a jet. The collection of the jet data and the processing of this data are discussed next. This is followed by a description of background and fiducial cuts needed before the jet cross section can be extracted. Finally, the procedure used to extract the jet cross section from the data is explained.

\section{A. Jet Definition}

In proton-antiproton $(p \bar{p})$ collisions, parton-parton scattering gives rise to jet production. Experimentally, the signature of a jet is a large energy deposit in a localized area of the detector. Theoretically and experimentally, the definition of a'jet is ambiguous.[21] Choosing an appropriate algorithm to find and measure the physical properties of jets in the detector will be discussed here. Jlow these jets and the measurement of their pliysical propertics are compared to theory will be discussed in the next chapter.

The CDF calorimetry is segmented in azimuth $(\phi)$ and in pseudorapidity ( $\eta$ where $\left.\eta \equiv-\ln \left(\tan \frac{\theta}{2}\right)\right)$, as shown in Figure 5. This type of segunentation forms towers which project from the renter of the detector where the collision nominally takes place outward. Most particles coming from the interaction region near the center of the detector shower in the calorimeters and are observed as towers of energy

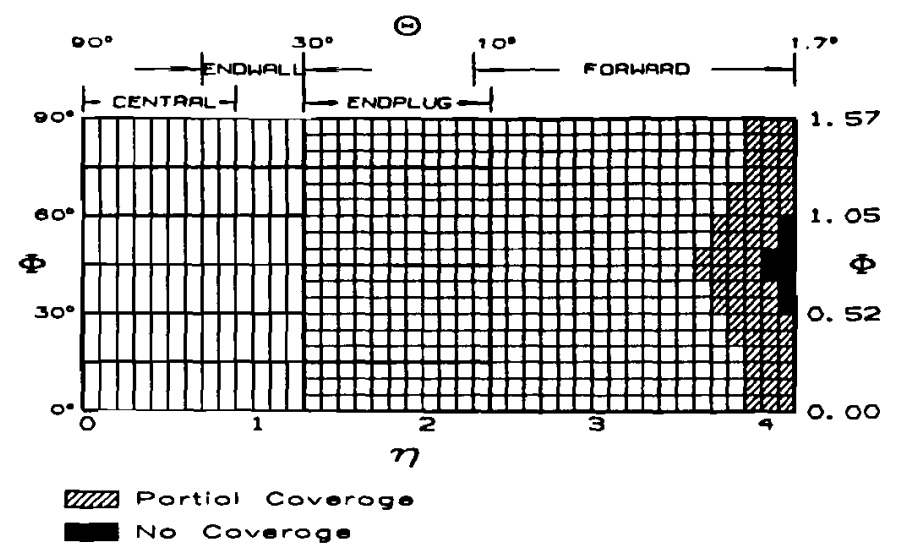

Figure 5. An example of the $\eta-\phi$ segmentation in one quadrant of the CDF hadronic calorimeter.

(see Figure 6). This projective geometry allows jets to be identified by algorithms which collect the towers into clusters of energy.

The types of algorithms typically used in $\bar{p} \bar{p}$ experiments are nearest neighbor $[45,46]$ and fixed cone. $(26,24]$ Each clustering algorithen starts with a list of seed towers. A seed tower is a single calorimeter tower with a transverse energy $\left(E_{t}\right.$, where $\left.E_{t} \equiv E \sin \theta\right)$ greater than some threshold $\left(E_{\mathrm{l}_{\text {ceed }}}\right)$.

In the nearest neighbor algorithm used in the trigger at CDF,[45] a list of candidate towers is then generated where a candidate tower is a calorimeter tower with $E_{l}$ above a second threshold $\left(E_{t_{c a n d}}\right)$. This algorithm then examines the 4 nearest neighbor towers (diagonal towers are not included, another algorithm examined by 


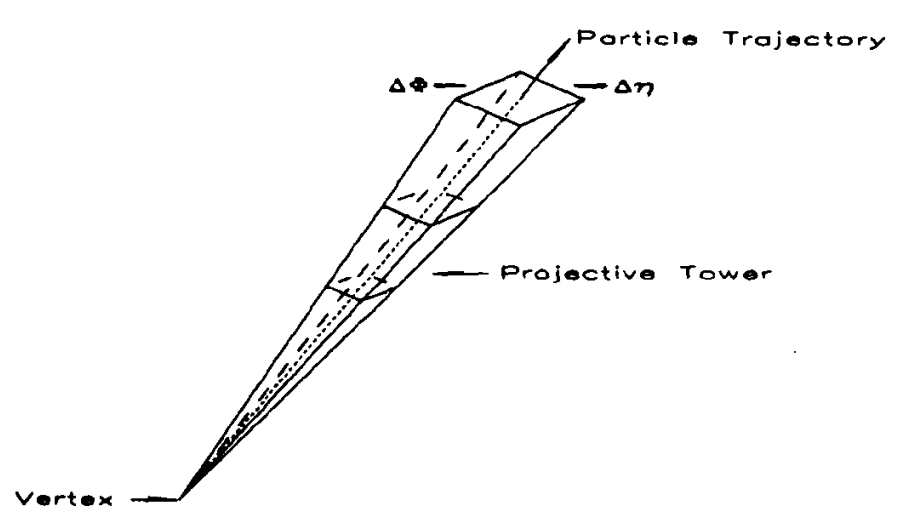

Figure 6. An illustration of a particle transversing a projective tower.

CDF used the 8 nearest neighbor towers) \{47\} of the seed tower and merges them with the seed tower if they are candidate towers. This process is then repeated using the candidate towers passing this requirement as seed towers until no new candidate towers are inerged. The whole procedure of finding jet clusters is repeated until no lurther seed towers exist.

Another type of algorithm examined by CDF is the variable cone algorithm. [47] For both the variable and fixed cone algorithms, the cone size $(R)$ in $\eta \phi$ space is defined to be $R=\sqrt{\Delta \eta^{2}+\Delta \phi^{2}}$. The variable cone algoritlum also forms a list of candidate towers as in the case of the nearest neiglibor algorithm. Candidate towers are then nerged with the seed tower if they are inside a cone of radius that varies as a function of $E_{t}$, where the $E_{t}$ is initially the $E_{t}$ of the seed tower. Then, for this algorithm, a new $E_{t}$ is calculated from the resulting merged cluster. The change in $E_{t}$ is then used to compute the ratio $\frac{\Delta E_{t}}{E_{1}}$, and if this ratio is greater than some fraction (0.02), a new cone size (which is now a function of jet $E_{t}$ ) is calculated and new candidate towers are inerged. This process is repeated until $\frac{\Delta E_{1}}{E_{1}}$ is less than the chosen ratio or the process of calculating $R$ has been repeated a maximum of five times. This procedure is again repeated until no more seed towers remain.

At CDF, jets are identified based on a fixed cone algorithn. This algorithm forms a list of seed and candidate towers as described above with $E_{t_{n, . .4}}=1.0 \mathrm{GeV}$ and $E_{i_{\text {cand }}}=1.0 \mathrm{GeV}$. Towers in the forward and endplug region (see Figure 5) are joined together in $\phi$ in order to correspond to the tower segmentation found in the central region. Next, preclusters arc formed from chains of contiguous towers that decrease in $E_{t}$, as in the ncarest neighbor algorithn (using the 8 nearest neighbors). The preclusters with $E_{t}$ greater than $1 \mathrm{GeV}$ are then used as starting points for clustering as shown in the flow chart in Figure 7. The $E_{t}$ weighted centroid of the precluster is found and a fixed cone in $\eta-\phi$ space is placed around this centroid. Candidate towers inside this cone are then merged together using the true tower segmentation (no joining in $\phi$ ). A new centroid is calculated and a new cone is drawn with this centroid as its center and a new list of merged towers is generated. This process is repeated until the list of merged towers in the cone remains unchanged. 
The procedure is repeated until no preclusters remain.

Overlapping jets, that is jets that contain all or part of another jet cluster, arc potentially difficult for any jet clustering algorithm. CDF handles this by comparing the amount of $E_{l}$ in towers common to both clusters to the amount of $E_{t}$ in the smaller cluster. If the ratio of these two numbers is larger than some cutoff $(0.75)$, the two clusters are merged into one cluster. If this ratio is smaller than this cutoff, then clusters are kept as separate jets and the towers in the overlap region are divided between the two jets based on their distance from the centroid of each cluster. New centroids are then computed for cach cluster and the original overlapping towers are then re-divided accordingly. This process is repeated until the list of towers in the clusters remains unclianged.

All of these algorithms were examined in detail for the analysis of CDF's 1987 data set.|47] 'The fixed cone algorithm was chosen over the other algorithms for several reasons, including the stability of this algorithun in measuring energy and position in the presence of other clusters, and the relative sharpness in $\eta-\phi$ space for its ability to distinguish between separate jets.

The various physical quantitics of the jet are characteristics which can be mcasured experimentally. CDF uses the following definitions for the cnergy, momentum, transverse momentum and transverse energy of the jet. The encrgy of each jet is defined to be the sum of the energies found in all the towers inside this cone,

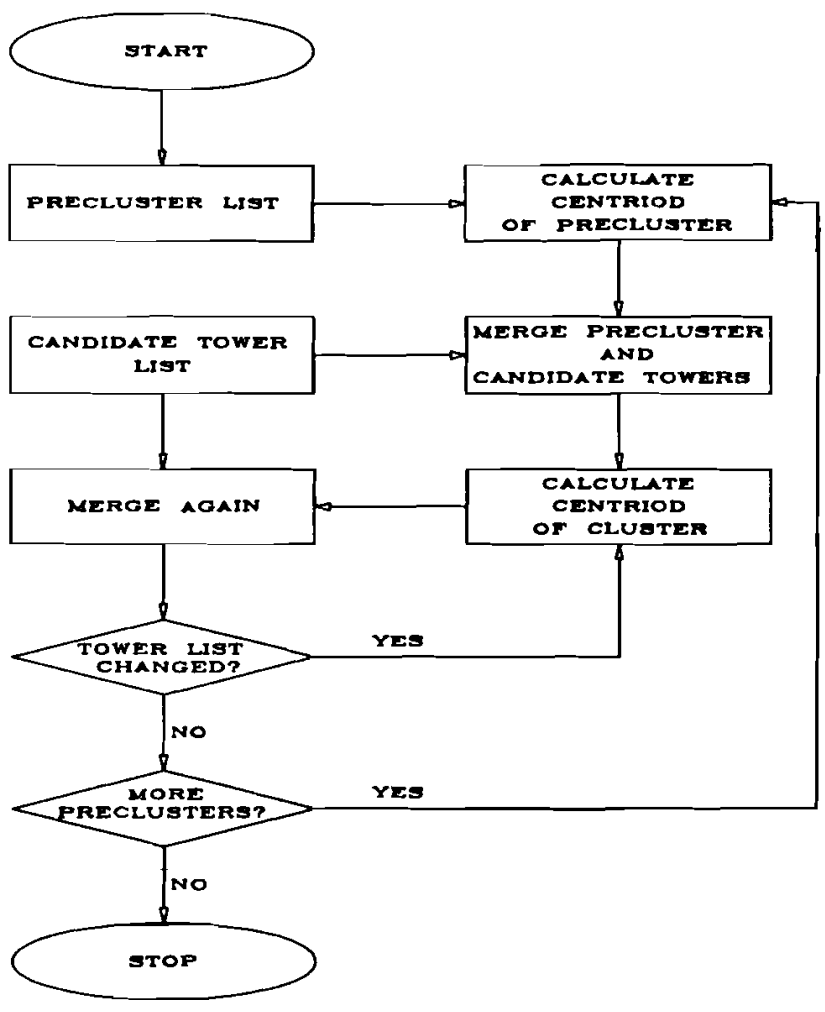

Figure 7. Flow chart of the fixed cone algorithm used by CDF, starting after candidate tower, seed tower and precluster lists have been formed. 
$E=\sum_{i=1}^{\# \text { lowers in cone }} E_{i}$. The momentun is calculated by assuming the center of each tower consists of massless particles and then defining:

$$
\begin{aligned}
& P_{z}=\sum_{i=1}^{\# \text { towers in cone }} E_{i} \cos \phi_{i} \sin \theta_{i} \\
& P_{y}=\sum_{i=1}^{\# \text { cowers in cone }} E_{z} \sin \phi_{i} \sin \theta_{i} \\
& P_{z}=\sum_{i=1}^{\text {towera in cone }} E_{1} \cos \theta_{i} \\
& |P|=\sqrt{P_{t}^{2}+P_{y}^{2}+P_{i}^{2}}
\end{aligned}
$$

where $E_{i,} \phi_{i}$, and $\theta_{i}$ are the energy, azimuth, and polar angle of the $i$ th tower. The transverse momentum $\left(P_{t}\right)$ and $E_{t}$, of the jet are defined as follows:

$$
\begin{aligned}
P_{t} & =\sqrt{P_{x}^{2}+P_{t}^{2}}, \\
E_{\ell} & =E\left(P_{t} /|P|\right), \\
& =E \sin \theta,
\end{aligned}
$$

where $E, P$, and $O$ are the energy, momentum, and polar angle of the jet.

The definition of a jet that CDF uses is compatible with that found in next-taleading order QCD inclusive jet cross section calculations done by S. Ellis, Z. Kunsat and D. Soper. $[21]$ One difference in the two algorithms is the definition of $E_{t}$. The next-to-leading order QCD calculation defines $E_{\mathrm{t}}=\sum_{\mathrm{i}} E_{\mathrm{i}} \sin \theta_{\mathrm{q}}$ whereas in the CDF aIgorithm $E_{l}=\sum_{i} E_{i} \sin \theta$, where $\theta$ is the polar angle for the $E_{l}$ weighted centroid of the jet. This difference is not expected to be larger than the theoretical uncertainties associated with the cross section. [21]
B. Data Collection and Triggering

Duting the period from August 1988 until June 1989, CDF collected $4 \times 10^{6}$ cvents corresponding to $4.2 \mathrm{pb}^{-1}$ of integrated lumninosity on 5,500 magnetic tapes. Collecting this amomnt of data required the ability to search the information recorded by the detector quickly for interesting events and to store these events on tape for later use. This was accomplished using a multiple level trigger. The trigger contained 4 different levels where each succeeding level reduced the amount of data by placing more stringent requirements on the data.

The first level (hevel 0) of triggering required a coincidence between the east and west beam-beam counters, as described in Chapter 1I. Events passing this requirement then went ont to the next level of triggering (Level 1). The Level 1 jet trigger summed (using the calorineter summer[45]) all trigger towers above $1 \mathrm{GeV}$ and required this sum to be either greater than 20 or $18 \mathrm{GeV}$, depending on which trigger was being used. Events passing the Level 1 trigger were then sent on to the Level 2 trigger.

The Level 2 trigger was the main level of triggering used for this jet measure. ment. Events in this trigger were required to contain at least one jet cluster with $E_{t}$ greater than 20,40 , or $60 \mathrm{GeV}$, where the 20 and $40 \mathrm{GeV}$ triggers were rale limited (pre-scaled). Initially, the pre-scale factora for the 20 and $40 \mathrm{GeV}$ triggers were 100 and 10 respectively. These pre-scale factors were chosen to keep the overall trigger ratc at around 1-2 $\mathrm{Hz}$, the rate at which the tape drives could be operated reliably. Tlis increases the efficiency for collecting the data by reducing the amount of time lost in writing data to magnetic tape. These faclors were changed to 300 and 30 later in order to decrease the amount of time lost collecting data when the luminosity of the 
beam grew larger. Jets clusters were found by the Level 2 cluster finder which used a nearest mighbor algorithm to cluster jets. This algorithm required a seed tower of greater than $3 \mathrm{GeV}$ and surnmed candidate towers of greater than $1 \mathrm{GeV}$. [48]

The Level 3 trigger was used to further filter the jet events. Level 3 is capable of executing algorithms written in FORTRAN 77, similar to the event reconstruction program used ofline to process the data.[49] This allowed algorithms which filtered noise and other backgrounds from the events to be run before recording the event to tape. This was only used during the later runs and required the event to have passed the Level 2 trigger before it was used. Since the same requirements of the Level 3 trigger were made when processing the data oflline, this trigger was equivalent to selecting events which passed the Level 2 trigger and had gone through oflline event reconstruction, except that it controlled which events were to be written to tape. Therefore, for this analysis an event was only required to pass the Level 2 trigger and to have gone through the event reconstruction processing.

\section{Event Reconstruction}

Analysis of the jet data begins with the reconstruction of the events stored on tape. The reconstruction program was used to filter noise from the data and remove events from individual runs with known detector problems. Sone of the sources of noise were pedestal shifts, bad electronics cards, "Texas 'Towers "(large energy spikes, thought to be associated with low energy neutrons first observed in the forward liadron calorimeter)[50], and cable noise on the cables connecting the detector to the electronics. Runs with known detector problens were runs with the ligh voltage turned off to part of the detector as well as runs with bad MET centering in the central detector (MET centering is the mean average missing $E_{t}$ calculated using minimum bias events) $[51,52]$. Reconstruction also took raw data and processed it into a format casily accessible to individual analysis prograns.

The process started by filtering the events according to trigger and then removing events which canne from runs with known detector problems. The event vertex was then reconstructed and raw calorimeter data was converted into energy. Next, filters were used to clean the events of noise $[53,54,55,56]$ and remove events containing cosinic rays not in coincidence with event crossings. [57) Tracks were reconstructed in the central tracking cliamber and jets were clustcred using the fixed cone algorithm (discussed in the first section of this chapter) with cone sizes of 0.4, 0.7 and 1.0. Fig. ure 8 is a flow cliart which illustrates the reconstruction path used for the jet data.

Data structures containing trigger, vertex, tracking and jet information were then written to special files for analysis. This climinated the need for the raw data and reduced the storage size of the data while inaintaining all the necessary information needed for the final analysis.

\section{Background Cuts}

To attempt to make any kind of a measurement of the jets themsclves, it was necessary to remove non-jet events from the data sample. Cosmic ray bremsstrahlung is one source of non-jet events. Thus, it was necessary to define criteria to identify these types of events and remove them from the event sample. To study the criteria 


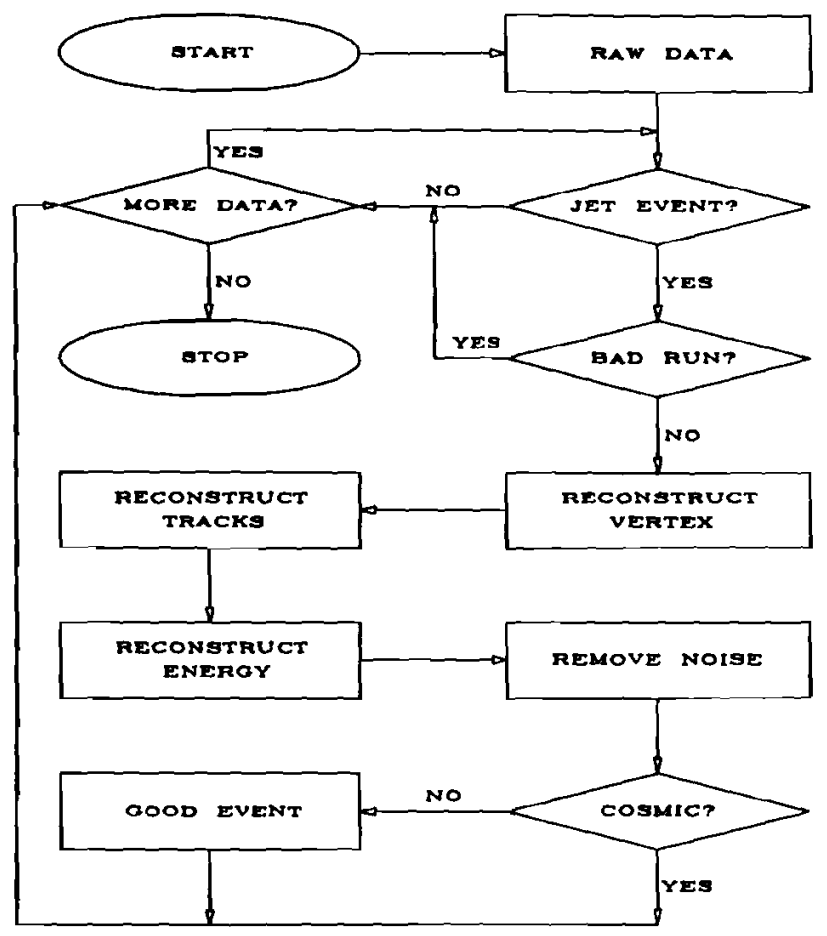

necrssary to remove these cosmic rays, a data set which consisted of $845 n b^{-1}$ (187260 events) of reconstructed data (with jets clustered using a cone size of 0.7 ) was used.

Three yuantities were used to deternitue the characteristics of an event in order to distinguish jet evcnts from background. [58, 59] Thesc quantities $E M F, C H F$, and MET will be described below. Cuts were made on these quantities and the events were classified according to which cuts they passed and which cuts they failed.

$\wedge$ jet is expected on average to have an electromagnetic fraction $(E M F)$ be. tween zero and one. Cosmic ray brenisstrahlifung, on the other hand, tends to leave energy in either the hadronic or electromagnetic calorimeter, thus having an $E M F$ near zcro or one. The EMF of the highest $E_{t}$ central jet was examined, but high encrgy photons or $\pi^{0}$ s also have an $E M F$ near zero or one thus $E M F$ was re-defined to be a more global quantity. $E M F$ was re-defined to be the $E_{t}$ weighted $E M F$ over the whole detector as follows:

$$
E M F=\frac{\sum_{j=1}^{N_{\mathrm{g} t}} E_{l_{j}} \times E M F_{j}}{\sum_{j=1}^{N_{j=1}} E_{l_{j}}},
$$

where $N_{\mathrm{jet}}$ is the number of jets with $E_{1}>5 \mathrm{GeV}, E_{\mathrm{t}}$ is the $E_{t}$ of the $j$ th jet and $E M F$, is the EMF of jet $j$. This re-definition of the EMF considers the event as a whole instcad of just one isolated jet in the event which may contain some interesting physics.

The value of this cut defined events with $0.1 \leq E M F \leq 0.95$ to be acceptable. Figure 9 shows the EMF for each event calculated by using the EMF of the highest $E_{t}$ central jet. Figure 10 shows the $E M F$ for each event as defined in Equation 14. The large spike observed in Figure 9 near zcro is a result of events which contained 


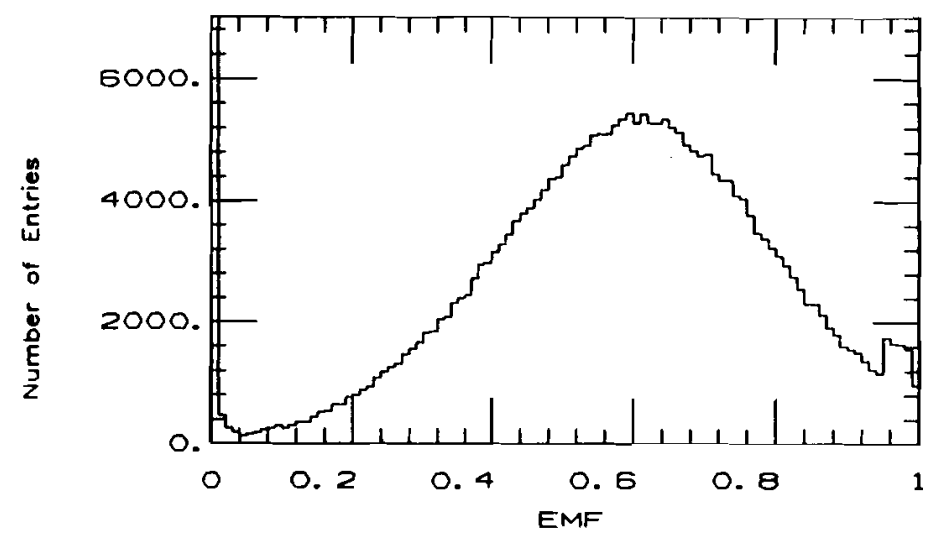

Figure 9. EMF of highest $E_{\ell}$ central jet in each event.

no jets above $10 \mathrm{GcV}$ in the central calorimeter. The tails of Figure 10 sliow that the cuts used are reasonable.

Partons originate from a $p \bar{p}$ collision and then emerge from the event vertex as a spray of both charged and nentral partictes which teave energy in the calorinuter anit are clustered into jets. Charged particle tracks associated with the jets, can then be reconstructed in the central tracking chamber. Cosmic rays and other backgrounds do not originate from the center of the detector and may or may not pass through the tracking chamber. Therefore, few if any tracks are found pointing at clusters of energy associated with these backgrounds. The next cut used was based on this premise and used the charged hadron fraction $(C H F)$ to determine whether the event may be a jet cvent or a non-jet event. The $C / I F$ of the event was calculated by first

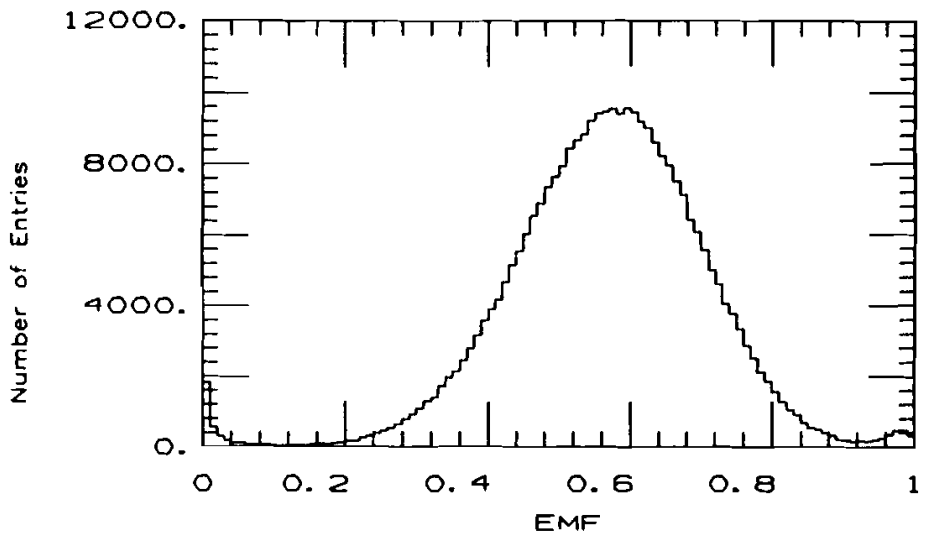

Figure 10. El weighted EMF' for each event (EMF).

finding all tracks pointing to each jet within a cone of 0.7 centered on the $\eta$ and $\phi$ of the jet. Since the tracking was only available in the central calorimeter, only the central area was used in this study. Therefore, there was an additional cut requiring Haclector $\mid \leq 0.7$, whefe Mdetertor is the pseudorapidity of the jet calculated, assuming a vertex of zero. CHIF was then calculated as follows:

$$
C H F=\frac{\sum_{j=1}^{N_{\mathrm{jet}}} \frac{\left(\sum_{i=1}^{*} \text { Tracks } P_{\mathrm{t}_{\mathrm{i}}}\right)_{j}}{E_{\mathrm{j}_{j}}}}{N_{\mathrm{jet}}},
$$

where $N_{\text {jet }}$ is the number of jets with $E_{t}>10 \mathrm{GeV},\left(\sum_{i=1}^{\# T_{\text {racks }}} P_{t_{1}}\right)$; is the sum of the $P_{t}$ of all the tracks in a cone of 0.7 around jet $j$ as measured by the tracking chamber, and $E_{l_{j}}$ is the $E_{t}$ of jet $j$ as measured by llie calorimeters. To pass this cut, the event was required to have $C / I F \geq 0.175$ in order to be accepted. Figure 11 


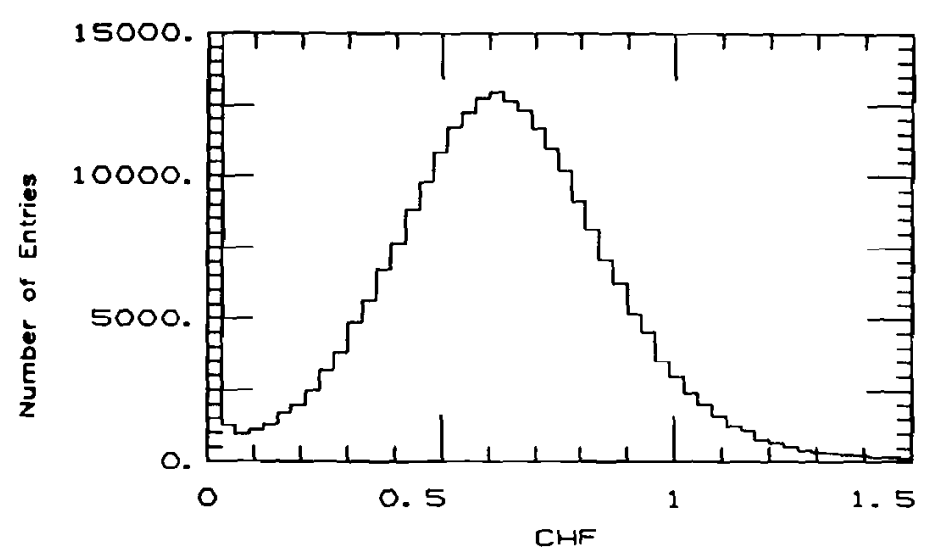

Figure 11. Average $P_{1} / E_{t}$ for all central jets in each event $(C H F)$.

shows this quantity as defined in Equation 15 for each event and shows that this cut is reasonable.

. The final cut used was based on Missing $E_{t}$ significance (MET). Physics re. quires momentum to be conserved. In particular, since the initial bean particles (protons, anti-protons) have little or no monentum transverse to the beam direction the total transverse momentum $\left(I_{T}\right)$ is expected to be small or zero from conserva tion laws. Typically, a jet event will have two jets which halance in $P_{t}$ (sce Appendix A). More specifically, for two jet events, the jets will be separated by approximately 180 degrees in azimuthal angle $(\phi)$ and will have approximately cqual amounts of $E_{t}$ MET is a measure of this balancing. Cosuic rays tend to deposit all their energy into one cluster and will have no balancing energy cluster, further, if they do manage

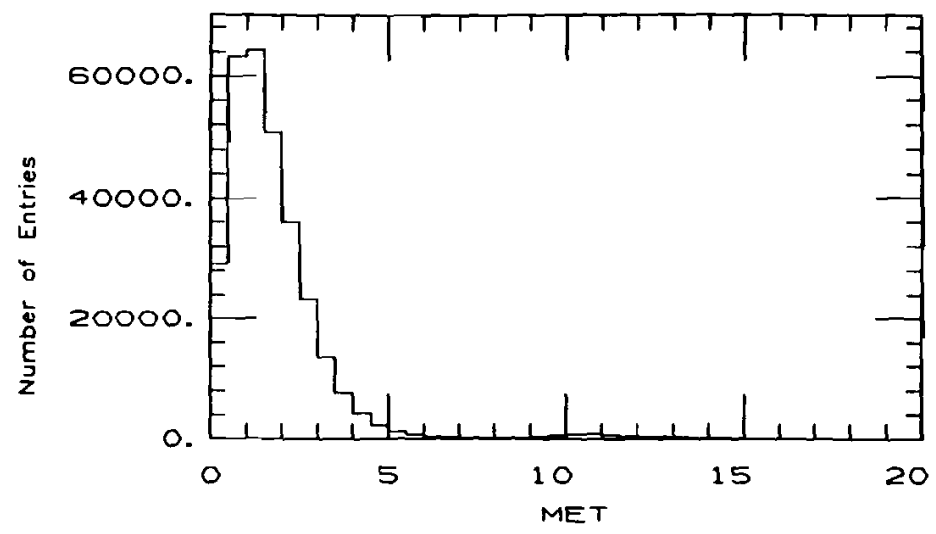

Figure 12. Missing $E_{\ell}$ significance for each event (MET).

to deposit energy into two separate clusters, these clusters tend not to be separated in $\phi$ by 180 degrces, hence they can be identified and removed. MET was calculated as follows:

$$
M E T=\frac{\left(\left(\sum_{j=1}^{N_{\text {jet }}} E_{t_{j}} \times \cos \phi_{j}\right)^{2}+\left(\sum_{j=1}^{N_{\text {jet }}} E_{t_{j}} \times \sin \phi_{j}\right)^{2}\right)^{\frac{1}{2}}}{0.8 \times\left(\sum_{j=1}^{\left.N_{\text {jet }} E_{t_{j}}\right)^{\frac{1}{2}}},\right.},
$$

where $N_{\text {jet }}$ was the number of jets with $E_{t}>5 \mathrm{GeV}$ and the event was considered acceptable if $M E T \leq 6.5$. Figure 12 shows the value of this quantity as defined in Equation 16 for caclı event.

The events were then classified based on these cuts as shown in Table IV. Because certain real physics processes can fail at least one of these cuts, and cosmic rays are expected to fail the majority of these cuts, events were required to pass at 
Table IV. This is the event classification scheme for determining backgrounds in jet events. All rlasses of events are shown. The numbering schente for the classes was for simplicity in coding

\begin{tabular}{ccc}
\hline \hline Class & Criteria & Category \\
\hline Class 0 & No Central Jets with $E_{t}>10 \mathrm{GeV}$ & Non-Central \\
Class 5 & $E M F<0.10, C H F>0.175, M E T>6.5$ & Cosinic \\
Class 6 & $E M F>0.95, C I I F>0.175, M E T>6.5$ & Cosnic \\
Class 11 & Failed 2 of 3 cuts & Cosmic \\
Class 21 & Failed 1 of 3 cuts & Jet Event \\
Class 31 & Failed 0 of 3 cuts & Jet Event \\
\hline \hline
\end{tabular}

least two of these cuts in order to be accepted as jel events. l'igures 13, 14, and 15 show correlations between $E M F, C M F$, and $M E T$. These figures show that the majority of the events tend to satisfy at least two of these cuts. While the rest of the events form isolated islands in these figures where the cosmic rays and other jet backgrounds are expected to be.[58]

The number of events in each Class is slown in Table $V$ for the events in the data sample used in this study. The 19 events will no classification are events which did not contain a jet cluster.

Most of the Class 5,6,11, and 21 events with at least one jet in the central calorirneter having an $E_{1} \geq 100 \mathrm{GeV}$ were then scanned. Scanning involved interactively examining each event using a display progran which slowed how the event looked in the detector. 'The calorimeter energy, tracks and muon hits were examined

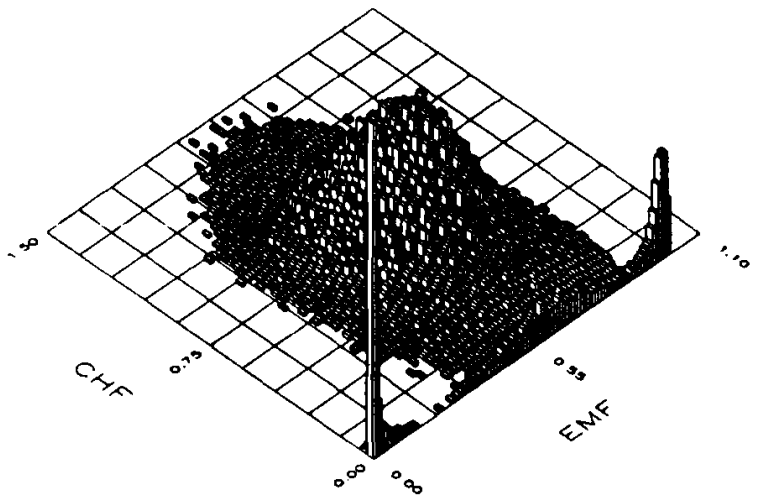

Figure 13. $C H F$ versus $E M F$.

in order to determine whether the event was background or not. All events in Class 21 and 31 with at least one jet in the central with $E_{t} \geq 175 \mathrm{GeV}$ were also scanned. (Note that events were not double counted for Class 21 events with $E_{t} \geq 175 \mathrm{GeV}$.) The results are summarized in Table VI

Table VI shows Class 11 was the place where the efficiency for distinguishing between jet events and background was the worst. However this Class only represents $0.09 \%$ of the total; thus by filtering on Classes 5,6 , and 11 only about $2.9 \%$ of these discarded central events were jet events while only $0.2 \%$ of events kept were cosmic rays.

Figure 16 shows the effective cross section for these background events as determined using the data set and cuts described above. This means discarding events in 


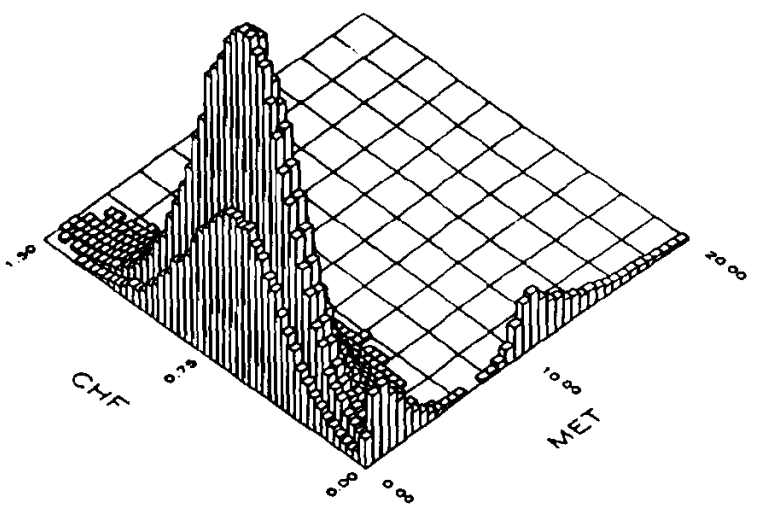

Figure 14. CHF versus $M E T$.

Classes 5,6 and 11 as background and only keeping events in Class 21 or 31. (Class 0 events are also discarded, not because they are cosmic background, but because they are non-central events.) Figure 17 shows the fraction of events discarded versus $E_{l}$. The expected cosmic ray background at $\mathrm{CDF}$ has been investigated[60] using a monte carlo simulation. The simulation included the effects of energy loss by cosmic rays due to bremsstrahlung, $e^{+} e^{-}$pair-production, and knock-on processes. The expected rates in the central hadronic calorimeter (CHA) and the central electromagnetic calotimeter (CEM) wete calculated. Then using Table VI, Figure 16 was scaled and compare this to these results. Figure 18 shows the results of this study.

From the results shown here, it has been dernonstrated that these cuts are very efficient in removing the cosmic background from the data sample. The agreement

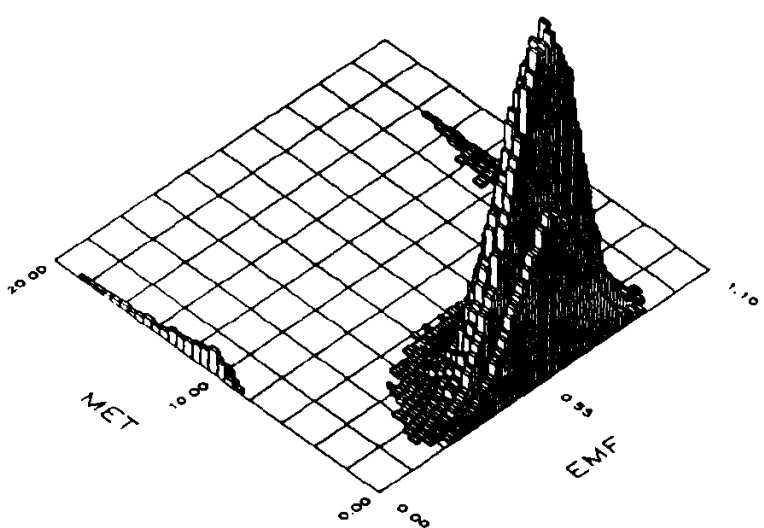

Figure 15. MET versus $E M F$.

between the predicted rates of cosmic rays with the rates found in the data shows these cuts are efficient at removing cosmic background from the data. In fact, the corrections for residual background contamination and for inefficiencies are so small that there is no reason to correct for this.

\section{E. Acceptance Region}

The calorimetry at CDF is broken up into three main detector regions, the central region with a pseudorapidity $(\eta)$ from $-1.3 \leq \eta \leq 1.3$, the plug region covering $1.3 \leq|\eta| \leq 2.4$, and the forward region for $2.3 \leq|\eta| \leq 4.2$. In the central region the calorimeter is made of scintillator and absorber, whereas in the plug and forward regions the calorimeters are gas proportional chambers. Gaps are 
Table V. Number of events per Class, found in the data sample used in this study.

\begin{tabular}{ccc}
\hline \hline Class & Events & Percent of Total (\%) \\
\hline Class 0 & 41098 & 21.95 \\
Class 5 & 430 & 0.23 \\
Class 6 & 276 & 0.15 \\
Class 11 & 175 & 0.09 \\
Class 21 & 3852 & 2.06 \\
Class 31 & 141410 & 75.51 \\
No Class & 19 & 0.01 \\
\hline Total & 187260 & 100.00 \\
\hline \hline
\end{tabular}

Table VI. Results from event scanning.

\begin{tabular}{cccccc}
\hline & \multicolumn{2}{c}{ Jet } & \multicolumn{2}{c}{ Cosrnic } \\
Class & Events & $\%$ & Events & $\%$ & Total Events \\
\hline Class 5 & 0 & 0.0 & 96 & 100.0 & 96 \\
Class 6 & 0 & 0.0 & 48 & 100.0 & 48 \\
Class 11 & $3-4$ & $11.1-14.8$ & $24-23$ & 88.9 .85 .2 & 27 \\
Class 21 & 102 & 100.0 & 0 & 0.0 & 102 \\
Class 21 and 31 & 400 & 99.8 & 1 & 0.2 & 401 \\
\hline
\end{tabular}

found in the regions that separate these various detector components. These gaps, combined with the different detector components, cause the jet resolution and the

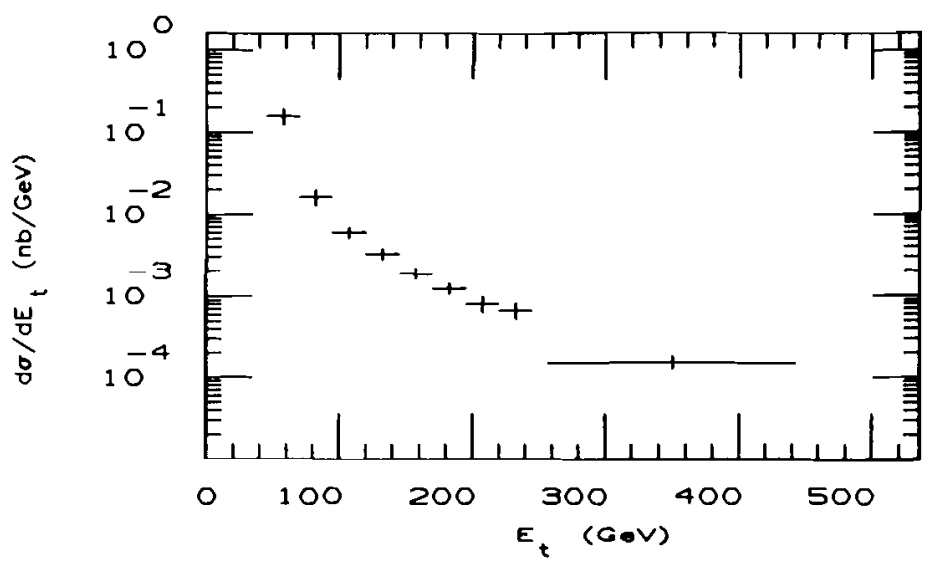

Figure 16. Effective cross section for jet backgrounds; Class 5, 6 and 11.

jet energy corrections to be dependent on the the $\eta$ of the jet. QCD predicts a fat spectrum at $\eta=0$ and the rate drops off by approximately $8 \%$ at $\eta=1.0 .[61]$

To insure full acceptance and a uniform detector response, the event vertex was restricted to be within $60 \mathrm{~cm}$ of the senter of the detector and the jets were restricted to have a detector pseudorapidity centroid of $0.1 \leq|\eta| \leq 0.7$, which is consistent with previous analyses. [26, 62]

Since the vertex cut reduces the number of events in the sample, the luminosity was corrected based on the vertex distributions for the data sample before and after these cuts. Figure 19 shows there are 243,100 events before this cut and there are 227,400 events after this cut. Therefore, the integrated luminosity was reduced by the ratio $227400 / 243100(0.94)$ to $3.9 p b^{-1}$ 


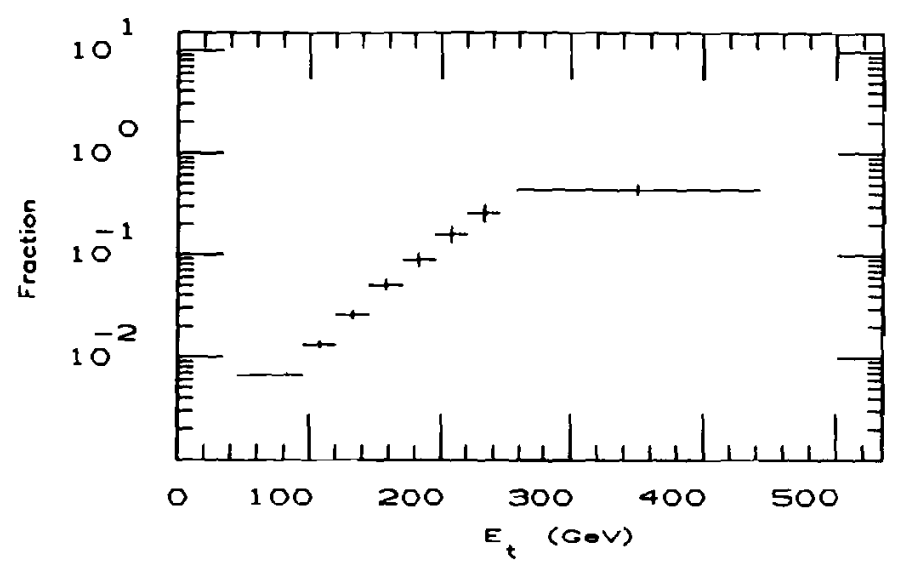

Figure 13. Fraction of jet backgrounds; Class 5,6 and II.

\section{F. Measuring the Raw Cross Section}

The raw jet cross section is the ineasured jet cross section which has not been corrected for the detector effects. The raw cross section was derived by collecting the jets in events passing the trigger, reconstruction, background, and fiducial cuts in $5 \mathrm{GeV}$ bins (for each trigger). (03) Jets from events passing triggers that were prescaled were scaled appropriately. The average $E_{t}$ was calculated for each bin and the number of entries in each bin was divided by the luminosity, bin size, and the pseudorapidity range (1.2). This gives a raw jet cross section which is nol corrected for jet resolution smearing and detector effects.

The raw cross sections for each trigger can then be compared to determine, for cach trigger, at what threshold the trigger becomes fully efficient. In the regions of
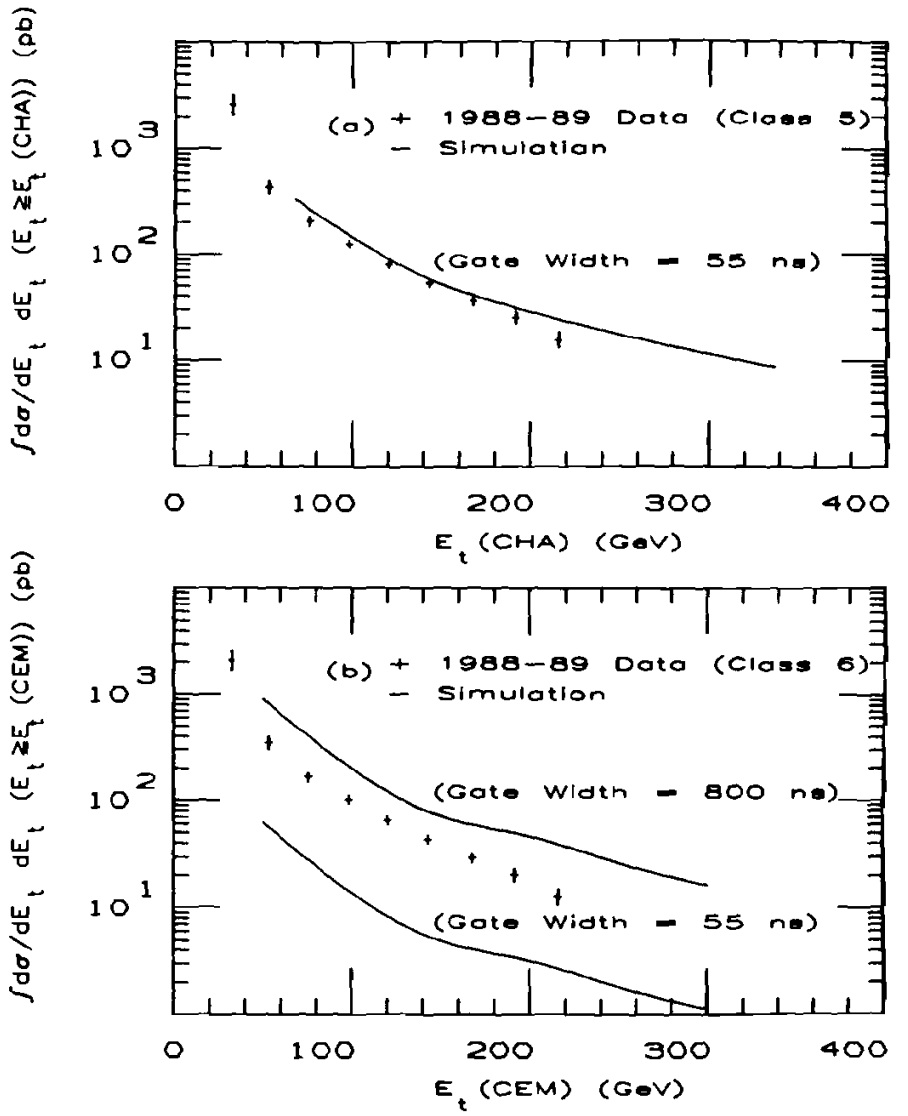

Figure 18. Expected cosmic ray event rales. Found in the a) CIIA, and b) CEM. 

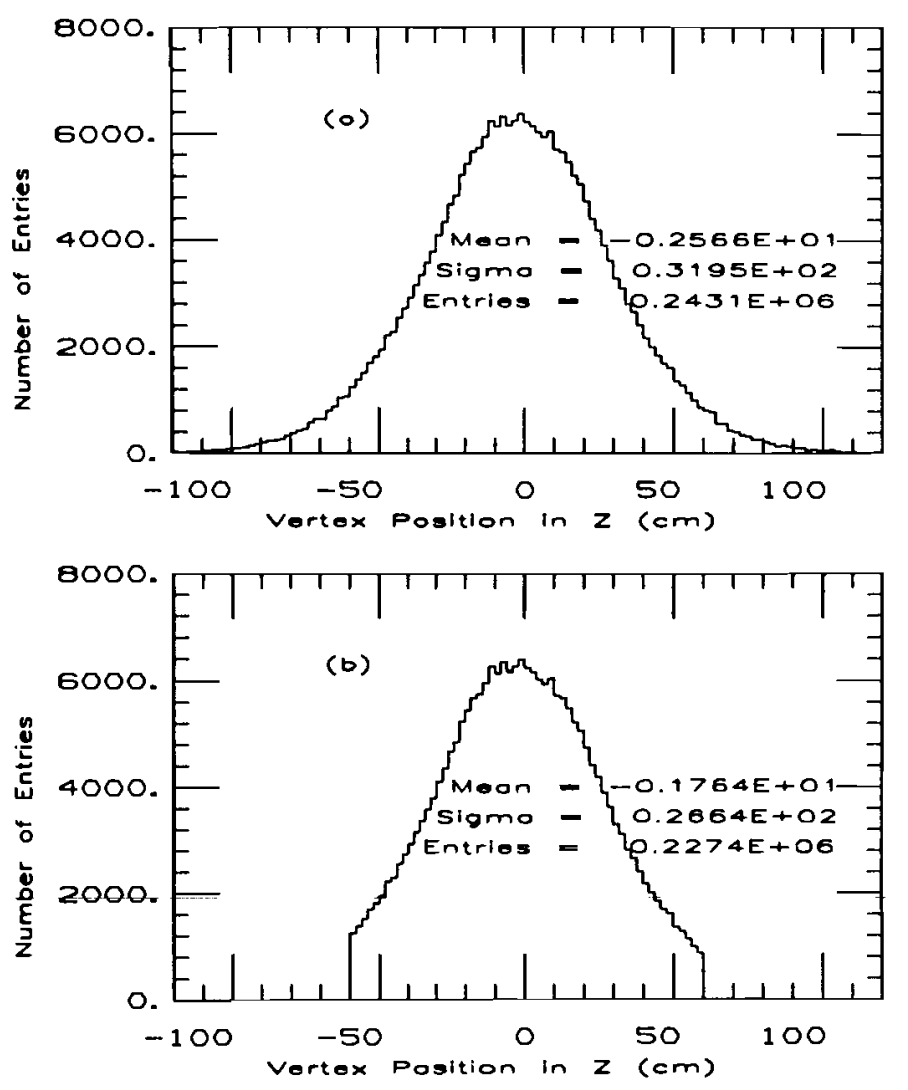

Figure 19. Event vertex distribution. Shown are the distributions found a) without and b) with the $60 \mathrm{~cm}$ vertex cut.
$E_{t}$ where the different triggers overlap, cuts were studied to determined the range where the triggers were fully efficient. These cuts were determined both as a function of trigger threshold and as a function of cone size. Figure 20 shows the comparison of the raw jet cross section for a cone size of 0.7 for the various triggers. A special 15 GeV trigger was uned in rompare to the $20 \mathrm{GeV}$ trigger. Table VII gives the effective cutoff threshold which was determined for each trigger as a function of cone size. This threshold is the point at which each trigger becomes fully efficient for each cone size.

The data below these thresholds were removed and the triggers combined to form the completed raw cross section. Bins which contained no events were merged with adjacent bins, and for bins containing fewer than 20 jets the statistical errors were determined using Poisson statistics. [64]

Using the procedures described in this chapter, the raw jet cross section has been measured for three cone sizes. The measured raw jet cross section for cone sizes of $0.4,0.7$, and 1.0 are listed in 'Tables VIII, IX, and X respectively. Also listed in these tables are the number of jets found in each bin scaled by the appropriate pre-scalc and the statistical error associated with the raw jet cross section. 


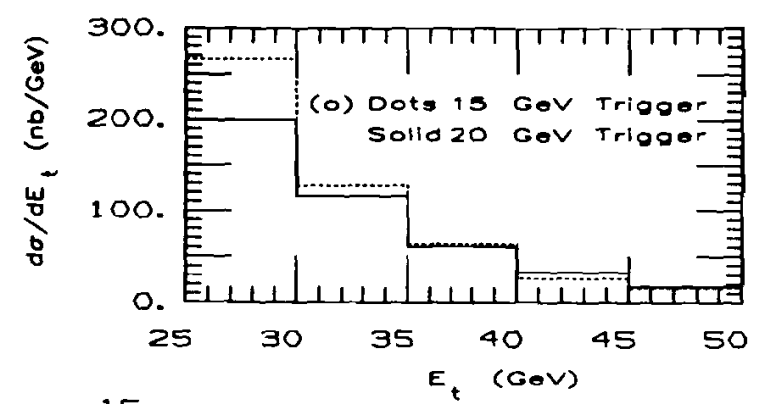

Table VII. Efficiency thresholds for jet triggers as a function of cone size.

\begin{tabular}{cccc}
\multicolumn{5}{c}{$\begin{array}{c}\text { Efficiency Threshold (GeV) } \\
\text { Cone Size 20 GeV trigger }\end{array}$} & 40 GeV trigger & 60 GeV trigger \\
\hline 1.0 & 45 & 70 & 110 \\
0.7 & 35 & 60 & 100 \\
0.4 & 30 & 50 & 90 \\
\hline
\end{tabular}
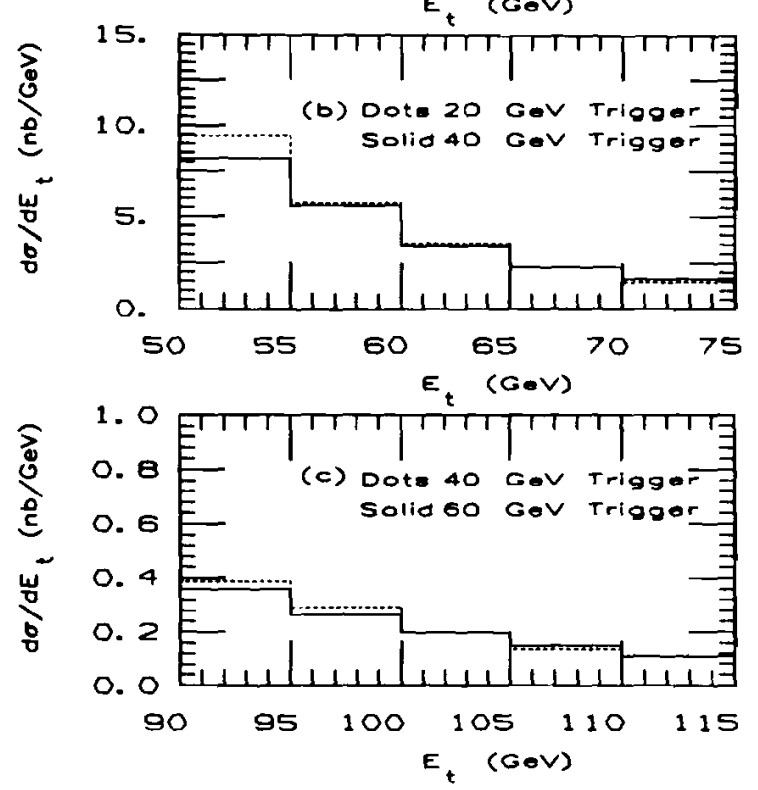

Figure 20. Comparisons of the raw trigger cross scctions for jets with a cone size of 0.7. The following triggers have been compared: a) $15 \mathrm{GeV}$ trigger compared to $20 \mathrm{GeV}$ trigger, a) $20 \mathrm{GeV}$ trigger compared to $40 \mathrm{GeV}$ trigger, a) $10 \mathrm{GeV}$ trigger compared to $60 \mathrm{GeV}$ trigger. 
Table VIII. Raw jet cross section and related quantities for a jet clustering cone size of 0.4 , number of jets has been scaled accordingly.

\begin{tabular}{|c|c|c|c|c|c|}
\hline \multicolumn{2}{|c|}{$\overline{\text { Bin Edges (GeV) }}$} & \multirow{2}{*}{$\begin{array}{c}\text { Mean } \\
E_{i}(\mathrm{GeV}) \\
\end{array}$} & \multirow{2}{*}{$\begin{array}{c}\text { Number of } \\
\text { Jets }\end{array}$} & \multirow{2}{*}{$\begin{array}{c}\text { Raw Cross } \\
\text { Section (nb/GeV) }\end{array}$} & \multirow{2}{*}{$\begin{array}{c}\text { Statistical } \\
\text { Error (nb/GeV) }\end{array}$} \\
\hline Lower & Upper & & & & \\
\hline 30 & 35 & 32.21 & 1775400 & 75.2 & \pm 0.974 \\
\hline 35 & 40 & 37.25 & 895500 & 37.9 & \pm 0.692 \\
\hline 40 & 45 & 42.28 & 447200 & 18.9 & \pm 0.489 \\
\hline 45 & 50 & 47.24 & 251700 & 10.7 & \pm 0.367 \\
\hline 50 & 55 & 52.29 & 151160 & 6.40 & \pm 0.0874 \\
\hline 55 & 60 & 57.33 & 93080 & 3.94 & \pm 0.0687 \\
\hline 60 & 65 & 62.30 & 57380 & 2.43 & \pm 0.0540 \\
\hline 65 & 70 & 67.41 & 38880 & 1.65 & \pm 0.0444 \\
\hline 70 & 75 & 72.42 & 26480 & 1.12 & \pm 0.0366 \\
\hline 75 & 80 & 77.31 & 17540 & 0.743 & \pm 0.0299 \\
\hline 80 & 85 & 82.37 & 12340 & 0.523 & \pm 0.0252 \\
\hline 85 & 90 & 87.32 & 9410 & 0.399 & \pm 0.0218 \\
\hline 90 & 95 & 92.37 & 6323 & 0.268 & \pm 0.00337 \\
\hline 95 & 100 & 97.36 & 4663 & 0.198 & \pm 0.00289 \\
\hline 100 & 105 & 102.32 & 3576 & 0.152 & \pm 0.00253 \\
\hline 105 & 110 & 107.31 & 2504 & 0.106 & \pm 0.00212 \\
\hline 110 & 115 & 112.50 & 1953 & 0.0827 & \pm 0.00187 \\
\hline 115 & 120 & 117.47 & 1472 & 0.0624 & \pm 0.00163 \\
\hline 120 & 125 & 122.43 & 1118 & 0.0474 & \pm 0.00142 \\
\hline 125 & 130 & 127.39 & 892 & 0.0378 & \pm 0.00127 \\
\hline 130 & 135 & 132.34 & 737 & 0.0312 & \pm 0.00115 \\
\hline 135 & 140 & 137.49 & 602 & 0.0255 & \pm 0.00104 \\
\hline 140 & 145 & 142.34 & 423 & 0.0179 & \pm 0.000871 \\
\hline
\end{tabular}

'T'able VIII. Continued

\begin{tabular}{|c|c|c|c|c|c|}
\hline \multicolumn{2}{|c|}{ Bin Elges (GeV) } & \multirow{2}{*}{$\begin{array}{c}\text { Mean } \\
E_{\ell}(\mathrm{GeV})\end{array}$} & \multirow{2}{*}{$\begin{array}{c}\text { Number of } \\
\text { Jets }\end{array}$} & \multirow{2}{*}{$\begin{array}{c}\text { Raw Cross } \\
\text { Section (nb/GeV) }\end{array}$} & \multirow{2}{*}{$\begin{array}{c}\text { Statistical } \\
\text { Error (nb/GeV) }\end{array}$} \\
\hline Lower & Upper & & & & \\
\hline 145 & 150 & 147.32 & 340 & 0.0144 & \pm 0.000781 \\
\hline 150 & 160 & 154.83 & 539 & 0.0114 & \pm 0.000492 \\
\hline 160 & 170 & 164.57 & 335 & 0.00710 & \pm 0.000388 \\
\hline 170 & 180 & 174.62 & 202 & 0.00428 & \pm 0.000301 \\
\hline 180 & 190 & 184.93 & 115 & 0.00244 & \pm 0.000227 \\
\hline 190 & 205 & 196.62 & 117 & 0.00165 & \pm 0.000153 \\
\hline 205 & 220 & 212.77 & 70 & 0.000989 & \pm 0.000118 \\
\hline 220 & 235 & 226.41 & 35 & 0.000494 & \pm 0.0000836 \\
\hline 235 & 250 & 243.51 & 28 & 0.000395 & \pm 0.0000747 \\
\hline 250 & 270 & 260.91 & 26 & 0.000275 & \pm 0.0000540 \\
\hline 270 & 290 & 281.68 & 8 & 0.0000847 & $\begin{array}{r}+0.0000419 \\
-0.0000293\end{array}$ \\
\hline 290 & 310 & 299.29 & 6 & 0.0000636 & $\begin{array}{r}+0.0000381 \\
-0.0000253\end{array}$ \\
\hline 310 & 330 & 319.53 & 3 & 0.0000318 & $\begin{array}{l}+0.0000310 \\
{ }_{-0.0000173}\end{array}$ \\
\hline 330 & 360 & 349.99 & 3 & 0.0000212 & $\begin{array}{l}+0.0000207 \\
-0.0000115\end{array}$ \\
\hline 360 & 405 & 385.92 & 2 & 0.00000942 & $\begin{array}{c}+0.0000125 \\
-0.00000610 \\
\end{array}$ \\
\hline
\end{tabular}


Table IX. Raw jet cross section and related quantitities for a jet clustering cone size of 0.7 , number of jets has been scaled accordingly.

\begin{tabular}{cccccc}
\hline \hline \multicolumn{7}{c}{ Bin Edges (GeV) } & \multicolumn{2}{c}{ Mean } & Number of & Raw Cross & Stalistica) \\
lower & Upper & $E_{1}(\mathrm{GeV})$ & Jets & Section (ub/GeV) & Error (nb/GeV) \\
\hline 35 & 40 & 37.25 & 1529200 & 64.8 & \pm 0.904 \\
40 & 45 & 42.20 & 800100 & 33.9 & \pm 0.654 \\
45 & 50 & 47.22 & 446800 & 18.9 & \pm 0.489 \\
50 & 55 & 52.33 & 236300 & 10.0 & \pm 0.356 \\
55 & 60 & 57.33 & 143500 & 6.08 & \pm 0.277 \\
60 & 65 & 62.30 & 87030 & 3.69 & \pm 0.0664 \\
65 & 70 & 67.31 & 57700 & 2.41 & \pm 0.0541 \\
70 & 75 & 72.33 & 41590 & 1.76 & \pm 0.0459 \\
75 & 80 & 77.31 & 27150 & 1.15 & \pm 0.0370 \\
80 & 85 & 82.36 & 18840 & 0.798 & \pm 0.0310 \\
85 & 90 & 87.30 & 13150 & 0.557 & \pm 0.0259 \\
90 & 95 & 92.31 & 9740 & 0.413 & \pm 0.0223 \\
95 & 100 & 97.36 & 7290 & 0.309 & \pm 0.0192 \\
100 & 105 & 102.39 & 4952 & 0.210 & \pm 0.00298 \\
105 & 110 & 107.36 & 3736 & 0.158 & \pm 0.00259 \\
110 & 115 & 112.37 & 2730 & 0.116 & \pm 0.00221 \\
115 & 120 & 117.41 & 2064 & 0.0874 & \pm 0.00192 \\
120 & 125 & 122.38 & 1581 & 0.0670 & \pm 0.00168 \\
125 & 130 & 127.35 & 1222 & 0.0518 & \pm 0.00148 \\
130 & 135 & 132.47 & 970 & 0.0411 & \pm 0.00132 \\
135 & 140 & 137.41 & 755 & 0.0320 & \pm 0.00116 \\
110 & 145 & 142.34 & 619 & 0.0262 & \pm 0.00105 \\
145 & 150 & 147.37 & 478 & 0.0203 & \pm 0.000926 \\
& & & & &
\end{tabular}

Table IX. Continued

\begin{tabular}{|c|c|c|c|c|c|}
\hline \multicolumn{2}{|c|}{ Biu Edges (GeV) } & \multirow{2}{*}{$\begin{array}{c}\text { Mean } \\
E_{t}(\mathrm{GeV})\end{array}$} & \multirow{2}{*}{$\begin{array}{c}\text { Number of } \\
\text { Jets }\end{array}$} & \multirow{2}{*}{$\begin{array}{c}\text { Raw Cross } \\
\text { Section (nb/GeV) }\end{array}$} & \multirow{2}{*}{$\begin{array}{c}\text { Stalistical } \\
\text { Error (nb/GeV }\end{array}$} \\
\hline Lower & Upper & & & & \\
\hline 150 & 160 & 154.62 & 711 & 0.0151 & \pm 0.000565 \\
\hline 160 & 170 & 164.42 & 475 & 0.0101 & \pm 0.000462 \\
\hline 170 & 180 & 174.70 & 280 & 0.00593 & \pm 0.000354 \\
\hline 180 & 190 & 184.64 & 156 & 0.00330 & \pm 0.000265 \\
\hline 190 & 205 & 196.34 & 161 & 0.00227 & \pm 0.000179 \\
\hline 205 & 220 & 211.73 & 98 & 0.00138 & \pm 0.000140 \\
\hline 220 & 235 & 226.08 & 56 & 0.000791 & \pm 0.000106 \\
\hline 235 & 250 & 243.19 & 29 & 0.000410 & \pm 0.0000761 \\
\hline 250 & 270 & 259.56 & 31 & 0.000328 & \pm 0.0000590 \\
\hline 270 & 290 & 278.95 & 16 & 0.000169 & $\begin{array}{c}+0.0000540 \\
-0.0000421\end{array}$ \\
\hline 290 & 310 & 298.70 & 7 & 0.0000741 & $\begin{array}{l}+0.0000400 \\
-0.0000274\end{array}$ \\
\hline 310 & 330 & 318.75 & 5 & 0.0000530 & $\begin{array}{l}+0.0000360 \\
-0.0000229\end{array}$ \\
\hline 330 & 360 & 353.57 & 3 & 0.0000212 & $\begin{array}{l}+0.0000207 \\
-0.0000113\end{array}$ \\
\hline 360 & 410 & 389.56 & 2 & 0.00000847 & $\begin{array}{r}+0.0000112 \\
-0.00000549 \\
\end{array}$ \\
\hline
\end{tabular}


Table X. Raw jet cross section and related quantities for a jet clustering cone size of 1.0 , number of jets has been scaled accordingly.

\begin{tabular}{|c|c|c|c|c|c|}
\hline \multicolumn{2}{|c|}{ Bin Edges $(\mathrm{GeV})$} & \multirow{2}{*}{$\begin{array}{c}\text { Mean } \\
E_{1}(\mathrm{GeV})\end{array}$} & \multirow{2}{*}{$\begin{array}{c}\text { Number of } \\
\text { Jets }\end{array}$} & \multirow{2}{*}{$\begin{array}{c}\text { Raw Cross } \\
\text { Section (nb/GeV) }\end{array}$} & \multirow{2}{*}{$\begin{array}{c}\text { Statistical } \\
\text { Error }(\mathrm{nb} / \mathrm{GeV})\end{array}$} \\
\hline Lower & Upper & & & & \\
\hline 45 & 50 & 47.30 & 712600 & 30.2 & \pm 0.617 \\
\hline 50 & 55 & 52.18 & 395500 & 16.8 & $\perp 0.460$ \\
\hline 55 & 60 & 57.30 & 238100 & 10.1 & \pm 0.357 \\
\hline 60 & 65 & 62.31 & 143100 & 6.06 & \pm 0.276 \\
\hline 65 & 70 & 67.51 & 97400 & 4.13 & \pm 0.228 \\
\hline 70 & 75 & 72.33 & 54790 & 2.32 & \pm 0.0526 \\
\hline 75 & 80 & 77.24 & 39550 & 1.68 & \pm 0.0448 \\
\hline 80 & 85 & 82.39 & 27000 & 1.14 & \pm 0.0370 \\
\hline 85 & 90 & 87.41 & 19280 & 0.817 & \pm 0.0313 \\
\hline 90 & 95 & 92.33 & 13190 & 0.559 & \pm 0.0259 \\
\hline 95 & 100 & 97.39 & 9830 & 0.416 & \pm 0.0223 \\
\hline 100 & 105 & 102.45 & 7090 & 0.300 & \pm 0.0190 \\
\hline 105 & 110 & 107.46 & 5170 & 0.219 & \pm 0.0162 \\
\hline 110 & 115 & 112.40 & 3826 & 0.162 & \pm 0.00262 \\
\hline 115 & 120 & 117.40 & 2815 & 0.119 & \pm 0.00225 \\
\hline 120 & 125 & 122.42 & 2177 & 0.0922 & \pm 0.00198 \\
\hline 125 & 130 & 127.37 & 1631 & 0.0691 & \pm 0.00171 \\
\hline 130 & 135 & 132.42 & 1254 & 0.0531 & \pm 0.00150 \\
\hline 135 & 140 & 137.39 & 1077 & 0.0456 & \pm 0.00139 \\
\hline 140 & 145 & 142.43 & 808 & $0.03 \cdot 12$ & \pm 0.00120 \\
\hline 145 & 150 & 147.46 & 646 & 0.0274 & \pm 0.00108 \\
\hline 150 & 160 & 154.78 & 931 & 0.0197 & $\pm 0.0006 \cdot 16$ \\
\hline 160 & 170 & 164.54 & 635 & 0.0135 & \pm 0.000534 \\
\hline
\end{tabular}

Table X. Continued

\begin{tabular}{|c|c|c|c|c|c|}
\hline \multicolumn{2}{|c|}{ Bin Edges (GeV) } & \multirow{2}{*}{$\begin{array}{c}\text { Mean } \\
E_{t}(\mathrm{GeV})\end{array}$} & \multirow{2}{*}{$\begin{array}{c}\text { Number of } \\
\text { Jets }\end{array}$} & \multirow{2}{*}{$\begin{array}{c}\text { Raw Cross } \\
\text { Section (nb/GeV) }\end{array}$} & \multirow{2}{*}{$\begin{array}{c}\text { Statistical } \\
\text { Error }(\mathrm{nb} / \mathrm{GeV})\end{array}$} \\
\hline Lower & Upper & & & & \\
\hline 170 & 180 & 174.76 & 377 & 0.00799 & \pm 0.000411 \\
\hline 180 & 190 & 184.50 & 219 & 0.00464 & \pm 0.000314 \\
\hline 190 & 205 & 196.69 & 212 & 0.00299 & \pm 0.000206 \\
\hline 205 & 220 & 212.30 & 126 & 0.00178 & \pm 0.000159 \\
\hline 220 & 235 & 225.88 & 72 & 0.00102 & \pm 0.000120 \\
\hline 235 & 250 & 241.99 & 37 & 0.000523 & \pm 0.0000859 \\
\hline 250 & 270 & 258.34 & 41 & 0.000434 & \pm 0.0000678 \\
\hline 270 & 290 & 280.24 & 19 & 0.000201 & ${ }_{-0.0000459}^{+0.0000577}$ \\
\hline 290 & 310 & 300.00 & 9 & 0.0000953 & $\begin{array}{l}+0.0000437 \\
-0.0000312\end{array}$ \\
\hline 310 & 330 & 320.63 & 4 & 0.0000424 & $\begin{array}{l}+0.0000337 \\
{ }_{-0.0000203}\end{array}$ \\
\hline 330 & 360 & 347.38 & 6 & 0.0000424 & $\begin{array}{l}+0.0000254 \\
-0.0000168\end{array}$ \\
\hline 360 & 410 & 393.87 & 4 & 0.0000169 & $\begin{array}{r}+0.0000135 \\
-0.00000813 \\
\end{array}$ \\
\hline
\end{tabular}




\section{CHAPTER IV}

\section{JET CORRECTIONS}

In order to compare the inclusive jet cross section to theory, the raw cross section needs to be cor rected for scveral effects. Energy degradation due to calorimeter effects and resolution smearing combined with the effect of a falling spectrum causes the jet cross section to be mismeasured. In order to correct for these effects, a detailed detector simulation has been employed to ascertain the magnitude of these effects. In conjunction with this, an unsmearing procedure was developed to correct the raw cross section for these effects. In the sections which follow, these procedures will be discussed in detail.

A. Simulation

The jet $E_{7}$ corrections used in previous CDF analyses were only calculated to $250 \mathrm{GeV}$. The 1988-1989 data set extends the cross section to $410 \mathrm{GeV}$ in $E_{t}$ therefore, it was necessary to recalculate these corrections and extend their range of validity. The jet resolution can also be extracted from the simulation at the same time.

In order to extract both $E_{t}$ corrections and resolution for jets in the CDF detector, a detector simulation was used to study the response of the detector to jets. A major ingredient of the jet $E_{t}$ corrections and jet resolution is the non-linearity in the response of the detector to charged particles. Therefore, the simulation was tuned to reproduce the single pion response observed in the test beam and the jet fraginentation observed in the data. In addition, the tracking efficiency in jets has been measured, parameterized, and introduced into the tracking simulation.

Much work has been done to tume the sinulation to reproduce the results of the test beam.[65] The simulation has been tuned to reproduce the observed single pion response, as wcll as the non-linearity observed at lower pion energies in minimum bias data. An increase in the amount of data available, compared to the previous (I)F collider run, has extended the momentum range covered in the tuning of the single pion response. 'This conbined with a redefinition of the calorimeter energy associated with the projected track has led to a substantial reduction in the systematic uncertainty associated with the neutral pion $\left(\pi^{0}\right)$ subtraction. It was therefore believed that if the simulation was tuned to reproduce the fragmentation observed in the data, the sinulation would give the correct result for the jet $E_{t}$ corrections and jet resolution.

A data set which consisted of approximately $4.2 \mathrm{pb}^{-1}$ of jet data in the region alove $120 \mathrm{GeV}$ in $E_{t}$, and approximately $871 n b^{-1}$ of jet data in the region from $30-120 \mathrm{GcV}$, was used to study the fragmentation.

The simulation was performed using the following analysis routines: SIMJET, SETPRT, QFLANA, JETCLU, QTKEFF, CENJET, and JETFRG.

1. SIMJET generated jets. It created two partons in a falling spectrum defined to match the spectrum measured in the inclusive jet cross section. The generated jets were also required to have $\eta>0.9$

2. SETPITT fragniented the jets. This routine has five adjustable parameters which were used to tune the fragmentation. During this study it was necessary to 
modify this routine to incorporate an $E_{l}$ dependence into one of these five variables.

3. QFLANA simulated the detector response based on the CDF detector geometry using generated particles as input and returning information on the encrgy in the calorimeter towers and tracks observed in the tracking chamber. This information could then be used to cluster jets. [66]

4. JETCLU clustered the simulated jets using the same algorithm as was used for the data.

5. Q'TKEFF incorporated tracking efficiency into the simulation ly flagging tracks as lost on the basis of a parameterization of tracking efficiency in jet events.

6. CENJE'T removed cosmic rays in the data and was therefore used on the simulation also.

7. JETFRG extracted and plotted the various fragmentation quantities examined in this study.

Since the simulation only supplied two partons, the data was requiresl to be two jet events. This was done by requiring the following criteria from the events.

1. There must be at least two jets.

2. There must be no third jet with $E_{t}>20 \mathrm{GeV}$.

3. The highest $E_{t}$ jet and the second highest $E_{l}$ jet inust be separated by at least 150 degrees in azimuthal angle $(\phi)$.

4. The $Z$ vertex was required to be witlin $60 \mathrm{~cm}$ of the center of the detector.

5. At least one jet was required to be in the central detector, $0.1 \leq|\eta| \leq 0.7$.
The jets were clustered with a cone sizc of 0.7 and tracks were required to be within the cone of the jet and to have $P_{t} \geq 500 \mathrm{MeV} / \mathrm{c}$. It should be noted that these same cuts were applied to both the simulation and the data.

The fragmentation clistributions observed in the data also includes the effects of tracking elficiency in jets. It is therefore necessary to measure the tracking efficiency in jets and apply this in the simulation. This work was done by $\mathbf{B}$. Winer and $P$. Tipton and was incorporated into QTKEFF.[67]

In order to compare the data and simulation, the following quantities were used to tune the fragmentation:

1. The fragmentation distribution (Z) defined in the following ways:

$$
\begin{aligned}
& Z(P)=P_{/ /}(\text {Track }) / P(J e t), \\
& Z(E)=P_{/ /}(\text {Track }) / E(J e t) \\
& Z(T)=P_{/ /}(\text {Traek }) / \sum_{\text {in cone }}(P(\text { Track }))
\end{aligned}
$$

$P_{/ /}$is the momentum of the track parallel to the jet axis. Each $Z$ was plotted as $\left(1 / N_{\text {,ets }}\right)(d N / d Z)$.

2. The number of tracks in the cone of the jet (Jet Multiplicity) was plotted as $\left(1 / N_{\text {jets }}\right)(M u l t)$

3. The number of tracks in the cone 90 degrees away in $\phi$ from the jet (Underlying Event Multiplicity) was plotted as $\left(1 / N_{\text {jets }}\right)\left(M u l U_{n d}\right)$.

4. The momentum perpendicular to the jet axis of tracks in the cone of the jet was plotted as $\left(1 / N_{\text {jets }}\right)\left(d N / d P_{\perp}\right)$, where $P_{\perp}$ is the momentum of the track perpendicular to the jet axis. 
5. For the tracks in a cone 90 degrees away in $\phi$ from the jet, the inonentum perpendicular to the axis of this cone was plotted as $\left(1 / N_{j e t s}\right)\left(d N / d P_{\perp} / n d\right)$.

6. The $P_{1}$ (relative to the beam axis) of the tracks in the cone around the jet was plotted as $\left(1 / N_{\text {jet }}\right)\left(d N / d P_{1}\right)$

7. The $P_{t}$ of the $t_{\text {racks }}$ in the cone 90 degrees away from the jet was plotted as $\left(1 / N_{\text {jets }}\right)\left(d N / d P_{t} U_{n d}\right)$

8. The $P_{1}$ flow of the tracks with respect to the lead jet in $\phi$, which is the $\Delta \phi$ of the track with respect to the lead jet weighted by the $P_{t}$ of the track.

9. The $P_{l}$ loss flow of the tracks with respect to the lead jet in $\phi$, which is the $\Delta \phi$ of the track witl respect to the lead jet weighted by the amount $P_{t}($ loss $)$ by the track due to the non-linearity.

10. The amount of energy loss in the jet due to the non-linearity in the central hadron calorimeter.

All of these variables were plotted as a function of jet $E_{\mathfrak{l}}$ for tuning any possible fragmentation evolution.

SETPRT contains five variables which can be used to tune the fragmentation of jets. These variables are labeled in SETPRT as follows: $X G E N(1), X G E N(2)$, $S I G Q T, S I G Q T 0$, and CON2. XGEN(1) and $X G E N(2)$ are used to describe the Field Feynman fragmentation parameterization[68) for jets in SETPRT (see Equation 20). The Field Feynman fragmentation is paramelerized by,

$$
F(Z)=1-X G E N(1)+X G E N(1) \times(X G E N(2)+1) \times(1-Z)^{X G E N(2)},(20)
$$

where $X G E N(1)$ essentially controls the nornalization and $X G E N(2)$ controls the slope of the fragmentation distribution. The fragmentation routine (SETPRT) does not include the effects of gluon radiation which distorts the fragmentation, therefore the transverse fragmentation necded to be tuned in order to match the data.[69] SIGQT0 is used to define the transverse $P_{1}$ of the tracks in the underlying event and $S I G Q T$ is used to define the transverse $P_{t}$ of the tracks in the jets as shown by the parancter $S / G$ in Equation 21. CON2 represents the transverse fragmentation power as described by Equation 21

$$
P_{1}=N * S I G * \sqrt{R^{-1 / C O N 2}-1}
$$

defines the $P_{1}$ distribution of the tracks in the event where $S I G$ modifies the normalization $N$ for the jet or underlying event and $\operatorname{CON} 2$ controls the slope for the distribution. The default values of these variables are listed in Table XI, along with the final tuned values. These five variables were tuned until there was agreement between the data and simulation for the plots listed above. This implies that the means, sigmas and shapes of each plot in the simulation was in agreement with the data. The simulation was tuned for jets with low $E_{1}$ ranging from $30-60 \mathrm{GeV}$ and also for jets with high $E_{t}$ ranging from $120-150 \mathrm{GeV}$.

After exalnining the simulation at higher $E_{i}$ 's in comparison with the data, it was necessary to make SIGQT a function of jet $E_{k}$. Knowing both the high (120$150 \mathrm{GeV}$ ) and low (30-60 GeV) $E_{t}$ tunes, SETPRT was then modified to scale SIGQT with jet $E_{1}$. These low and high values are also listed in Table XI. 
Table XI. Values of the tuned parameters in SETPRT. Including the values of the default parameters and both low (30-60 GeV) and high (120-150 GeV) tune values for the unmodified version of SET'PRT.

\begin{tabular}{ccccc}
\hline Variable & Default Value & Low Value & IIigh Value & Final Tune \\
\hline XGEN(1) & 0.950 & 0.950 & 0.950 & 0.950 \\
XGEN(2) & 7.000 & 7.500 & 7.500 & 7.500 \\
SIGQT & 0.275 & 0.275 & 0.600 & 0.250 \\
SIGQT0 & 0.370 & 0.370 & 0.370 & 0.370 \\
CON2 & 1.100 & 1.200 & 1.200 & 1.200 \\
\hline
\end{tabular}

With the final modifications to SETP'RT, the fragmentation was tuncd in SET PRT to agree with what was observed in the CDF jet data over the full range of jet $E_{i}$ 's. In order to check the fraginentation at low $E_{t}$, an unbiased data sample was needed. This was obtained by using the jets in the photon data sample which had a lower trigger threshold. The results of the fragmentation observed in jets ranging from $10-20 \mathrm{GeV}$ in the photon data sample were then compared to the simulation. As can be seen in Figures 21, 22, 23, and 24, the various fragmentation plots in both simulation and data agree at both low and high $E_{t}$.

The jet fragmentation was also tuned to model the non-linearity of the hadronic calorimeter since the main source of energy loss in jets are low energy particles. Figure 25 shows the percent of energy loss due to the non-linearity in the central hadronic calorimeter for both the data and simulation. This energy loss in the simulation and the data agree at the level of $1-3 \%$. This agreenent combined with the tuning of
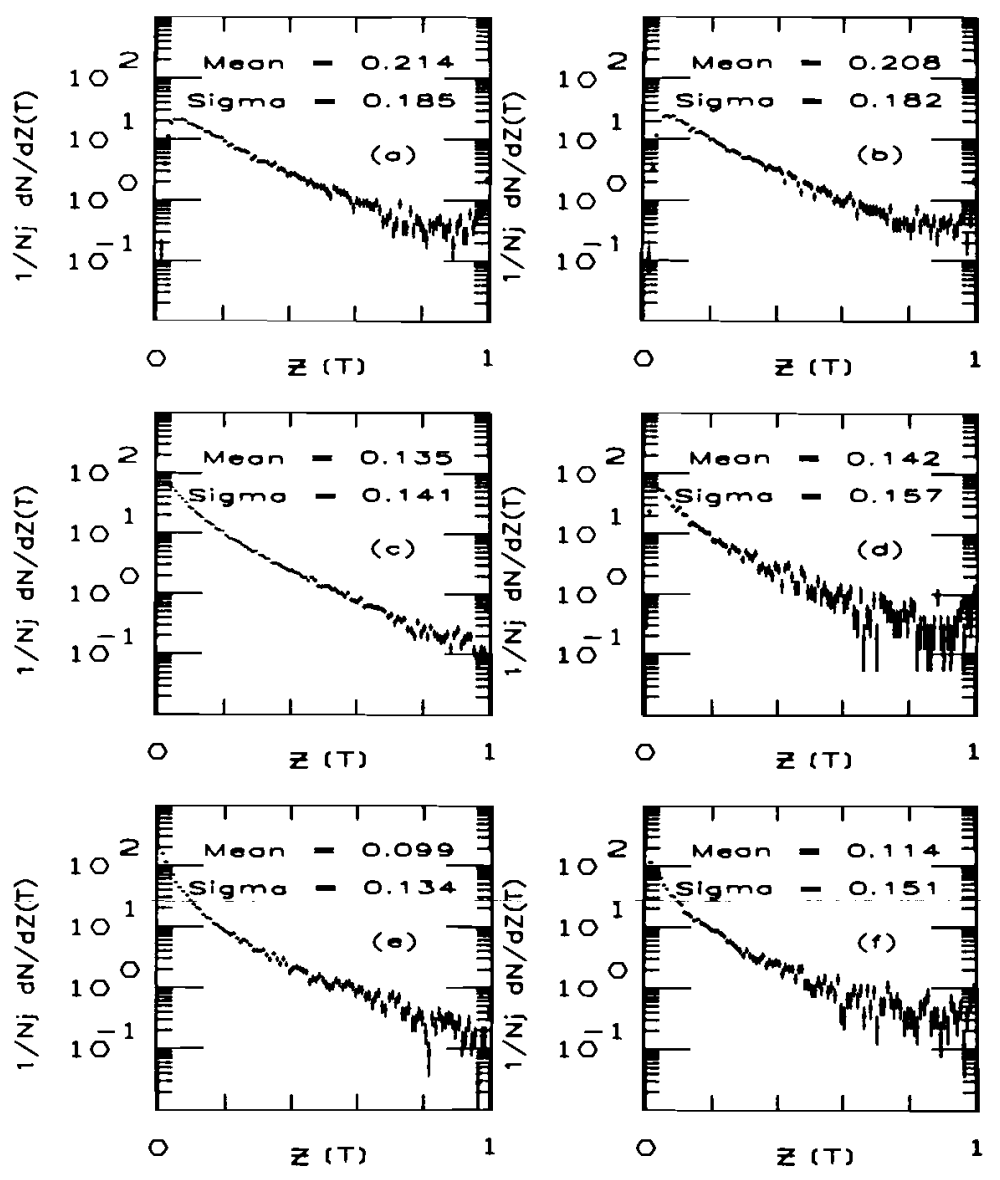

Figure 21. The distribution of $Z(T)$ in jets with a cone size of 0.7 as defined in Equation 19. Shown are the a) data and b) simulation in the $E_{t}$ range $10.20 \mathrm{GeV}, \mathrm{c})$ data and d) simulation in the $E_{1}$ range $30-60 \mathrm{GeV}$, e) data and f) simulation in the $E_{t}$ range 120-150 GeV. 

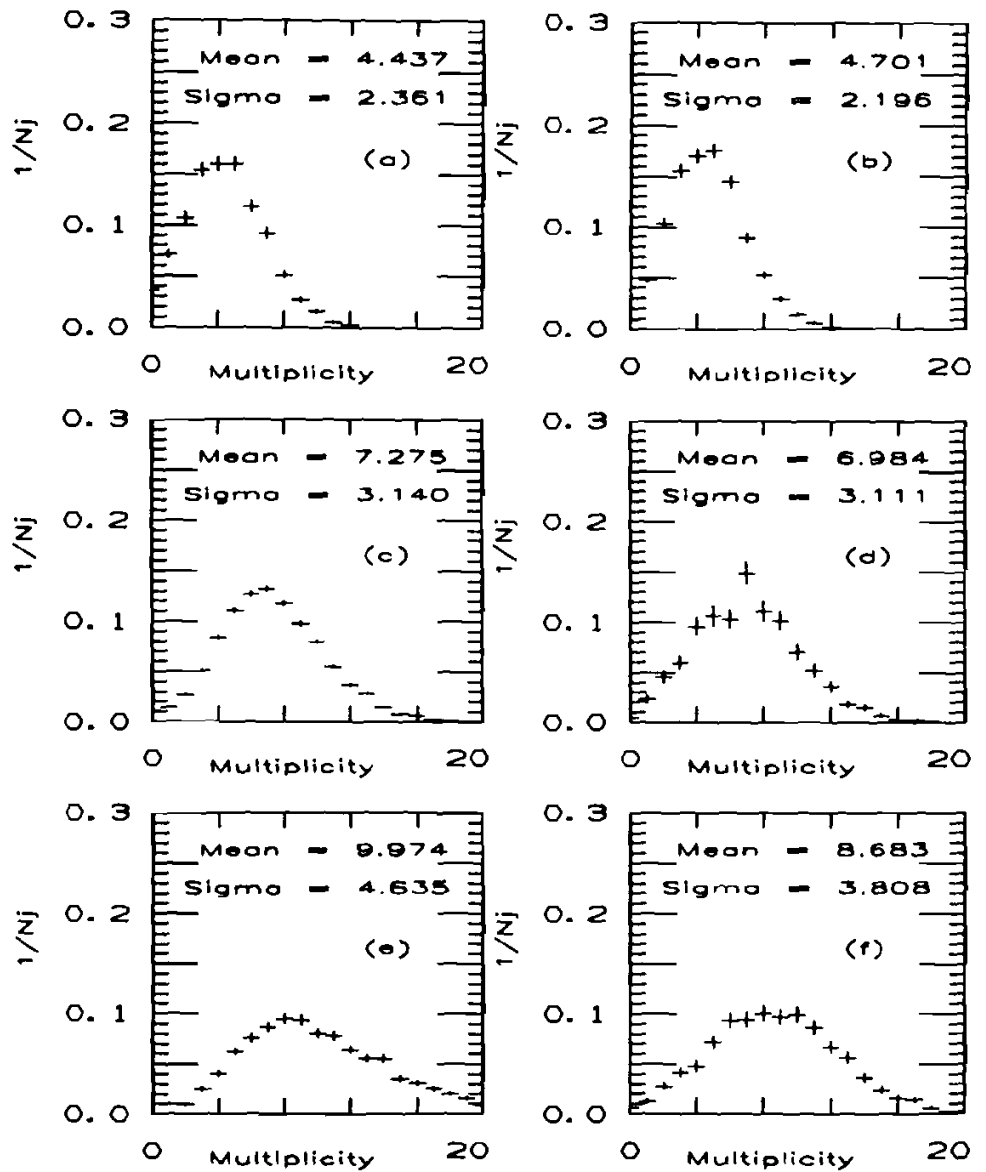

Figure 22. The jet track multiplicity in jets with a cone size of 0.7 . Shown are the a) data and b) simulation in the $E_{t}$ range $\left.10-20 \mathrm{GeV}, c\right)$ data and d) simulation in the $E_{t}$ range $\left.30-60 \mathrm{GeV}, e\right)$ data and $\mathrm{f}$ ) simulation in the Et range 120-150 GeV.
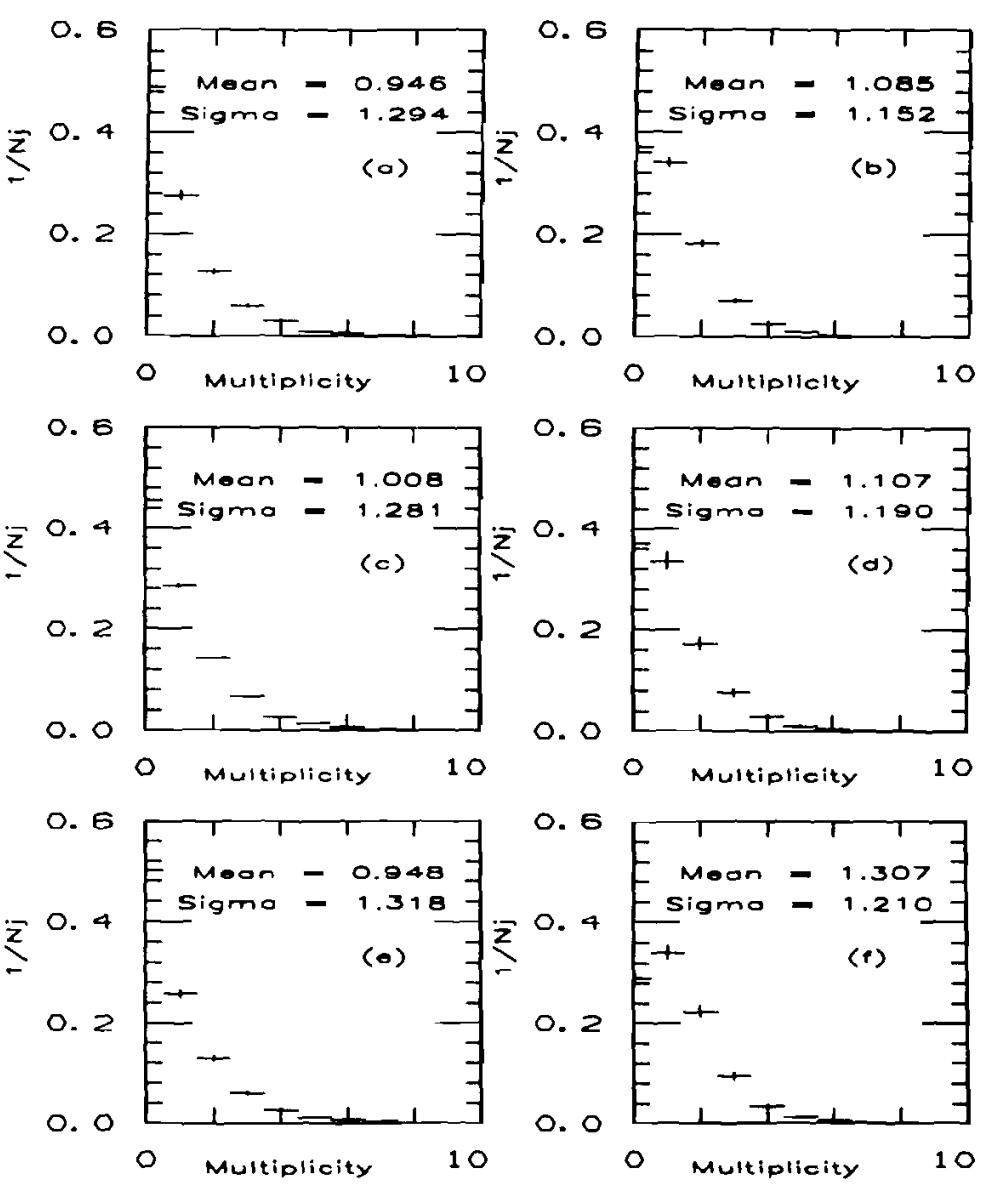

Figure 23. The underlying event track multiplicity in jets with a cone size of 0.7 . Shown are the a) data and b) simulation in the $E_{t}$ range $10-20 \mathrm{GeV}$, c) data and d) simulation in the $E_{\ell}$ range $30-60 \mathrm{GeV}$, e) data and f) simulation in the $E_{1}$ range $120-150 \mathrm{GeV}$ 

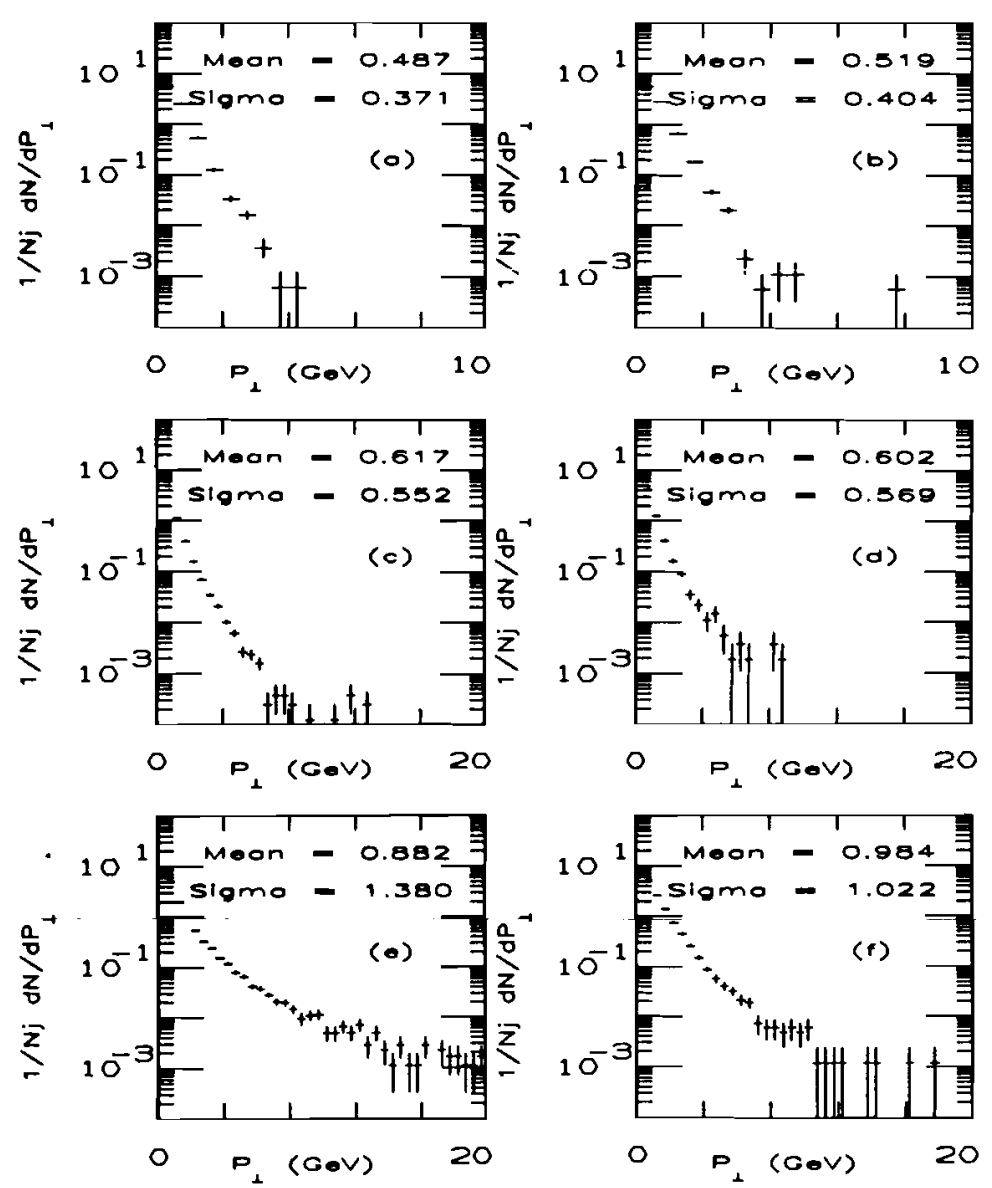

Figure 24. The distribution of $P_{\perp}$ in jets with a cone size of 0.7. Shown are the a) data and b) simulation in the $E_{t}$ range $10-20 \mathrm{GeV}$, c) data and d) simulation in the $E_{t}$ range $30.60 \mathrm{GeV}$, e) data and f) simulation in the El range $120-150 \mathrm{GeV}$. the single pion response, shows both jet $E_{t}$ corrections and jet resolution can be accurately extracted from the simulation out to $450 \mathrm{GeV}$ in $E_{t}$

B. Jet $E_{t}$ and Resolution

In this section the procedure used to obtain the corrected inclusive jet $E_{t}$ cross section from the measured spectrum is described. In order to have a well defined method for accounting for the effects of both jet resolution and $E_{t}$ degradation, the $E_{\mathfrak{l}}$ corrections and jet resolution have been combined into a single unsmearing procedure. [70]

In order to measure the inclusive jet cross section, the data was first placed in bins of measured (uncorrected) $E_{t}$. A given jet of true transverse energy ( $E_{t}^{\text {rue }}$ ) may contribute to a bin of measured $E_{t}$ ( $\left.E_{t}^{\text {meas}}\right)$ because of energy degradation or calorimeter measurement error (resolution). Therefore, the $E_{\ell}$ and resolution corrections to thic observed jet cross section are coupled and corrections to the cross section must account for this coupling. Traditionally, jet $E_{t}$ corrections (obtained through detector simulation) have been based on the relationship between the true jet $E_{t}$ and the average $E_{t}^{\text {meas }}$ associated with that true jet; this is an inappropriate correction to apply when jet resolution effects contribute substantially to $E_{t}^{\text {meas. }}$. Therefore, $E_{t}$ and rcsolution effects have been combined in the unsmearing of the inclusive jet cross section.

In order to begin the process of unsmearing the cross section, the jet response function for all valucs of $E_{t}^{\text {true, }}$, namely the set of measured jet $E_{\mathfrak{t}}$ distributions associated with each $E_{4}^{\text {true, }}$, were obtained. These response functions, which reflect both 

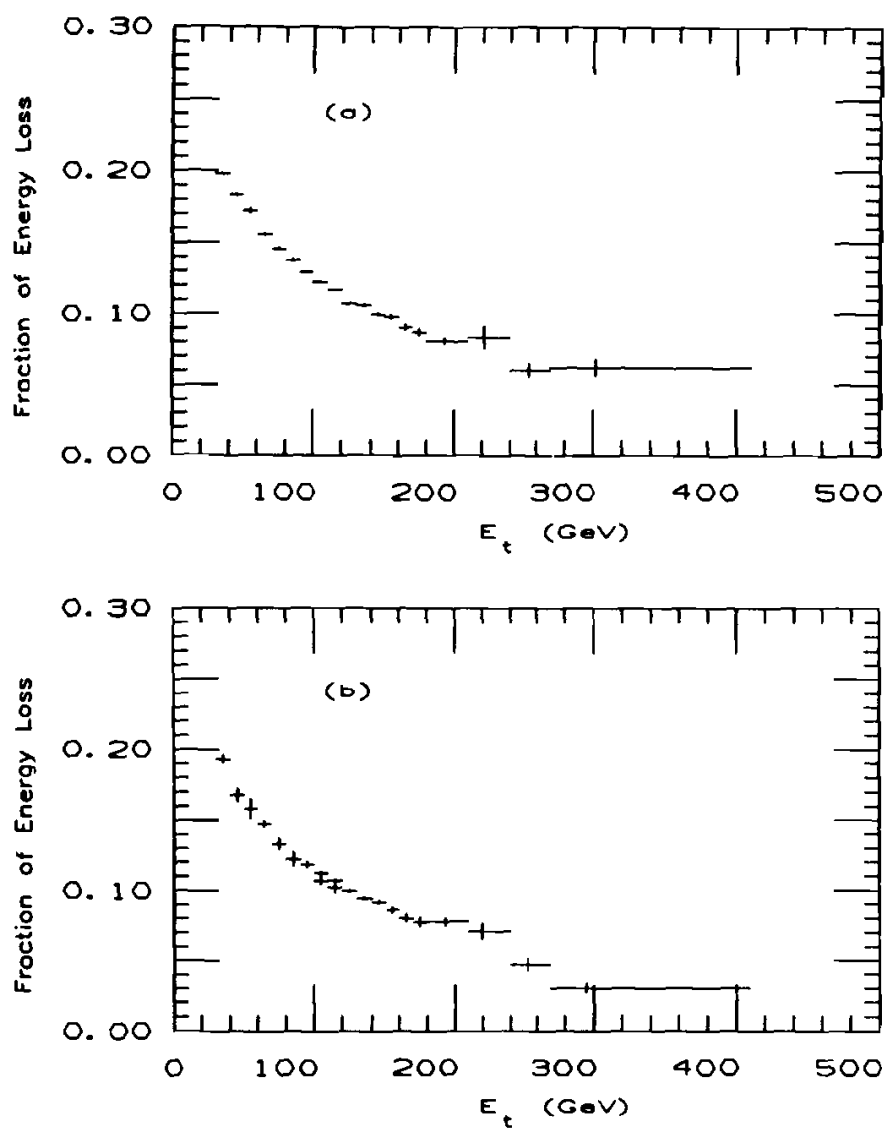

Figure 25. Fraction of energy loss in jets with a cone size of 0.7 , due to hadronic ton-linearity. This energy loss is plotted for both the a) data and b) simulation.

degradation and resolution, were found using a detector sinulation tuned to reproduce single-particle calorimeter response[65] and jet fragmentation propertics as discussed earlier. Dijet events with a flat $E_{i}^{\text {true }}$ spectrum were generated without $k_{t}$ smearing (which is a smearing of the momentmm balance in $t$ wo jet events). $k_{t}$ smearing was not used since this is sensitive to QCD effects of both soft and hard gluon emission and detector effects, and the corrections for detector effects needed to be isolated. The cvents were generated in the $E_{t}$ interval of $0-900 \mathrm{GeV}$, and a scatterplot of Eqeas vs $E_{l}^{\text {true }}$ was made. (Jets were also required to impact the caloritneter in the detector- $\eta$ interval $0.1<\left|y_{d}\right|<0.7$, the same requirement was inade for the data.) $E_{t}^{\text {true was }}$ defined to be the sum of all gencrated particles which point into the measured jet clustering cone associated with the reconstructed jet. Only particles coming from the parton closest in $\eta$ and $\phi$ were considered in the sum, and no correction was made for energy lost outside the conc. 'This was done in order to be able to compare the final cross section to uext-to-leading order QCD calculations where a correction for cnergy lost outside the clustering cone is no longer valid.[21]

Using this scatterplot, distributions of $E_{t}^{\text {meas }}$ were extracted for various val.

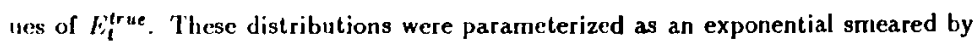
a Gaussian: paranneters in the fits were (1) the average of the $E_{i}^{\text {meas }}$ distribution (Mean), (2) the decay constant of thie exponential (Slope), and (3) the standard deviation of the Gaussian (Sigma). Typical fits of this parameterization for a measured jet cone size of $0.4,0.7$, and 1.0 evaluated at $E_{t}^{\text {true }}=20,50$, and $200 \mathrm{GeV}$ can be seen in Figure 26. The Mean, Slope, and Sigma were then parameterized as a function 
of $E_{t}^{\text {rue, }}$, thus defining the jet response functions for an $E_{t}$ range from $0900 \mathrm{GeV}$. The response functions predicted from detector simulation were found to have upward going non-Gaussain tails for $E_{l}^{\text {true's }}$ below about $50 \mathrm{GeV}$, were nearly Gaussian in the interval 50-80 GeV, and developed downward going non-Gaussain tails above about $80 \mathrm{GeV}$. These tails in the distributions are associated with the effect of the non-linearity for low energy pions in the hadron calorimeter.

Jet response functions were measured for cone sizes of $0.4,0.7$, and 1.0 ; the following parameterizations were then used for Mean, Sigma, and Slope;

$$
\begin{aligned}
& \text { Mean }=A E_{t}^{3}+B E_{t}^{1 / 3}+C E_{t}^{2}+D E_{t}^{1 / 2}+E E_{t}+F, \\
& \text { Sigma }=W\left(E_{t}+X\right)^{\gamma}+Z \\
& \text { Slope }=M E_{t}+N\left(\text { for } E_{t}<\text { CUTOFF }\right), \\
& \text { Slope }=Q E_{t}+R\left(\text { for } E_{t} \geq \text { CUTOFF }\right),
\end{aligned}
$$

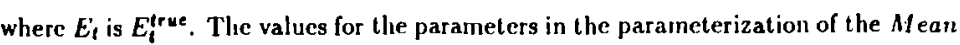
are listed in Table XII. The values of the parameters in the parameterization of Sigma are listed in Table XIII and the values of the parameters in the parameterization of Slope are listed in Table XIV.

The contribution of the cluster-finding efficiency to the response functions has also been considered. The cluster-finder efficiency can be included in the response functions, $R\left(E_{l}^{\text {true }}, E_{l}^{\text {meas }}\right)$, by scaling them by this efficiency. If $E f f\left(E_{l}^{\text {true }}\right)$ is the fraction of time that a true jet of $E_{l}^{\text {free }}$ leaves no cluster, the new response function is redefined as $R\left(E_{t}^{\text {true }}, E_{t}^{\text {meas }}\right)=\left|1-E f f\left(E_{t}^{\text {true }}\right)\right| \times R\left(E_{t}^{\text {true }}, E_{t}^{\text {neas }}\right)$. The results from photon-jet balancing studies $[31]$ were used to determine the size of $E f f$.
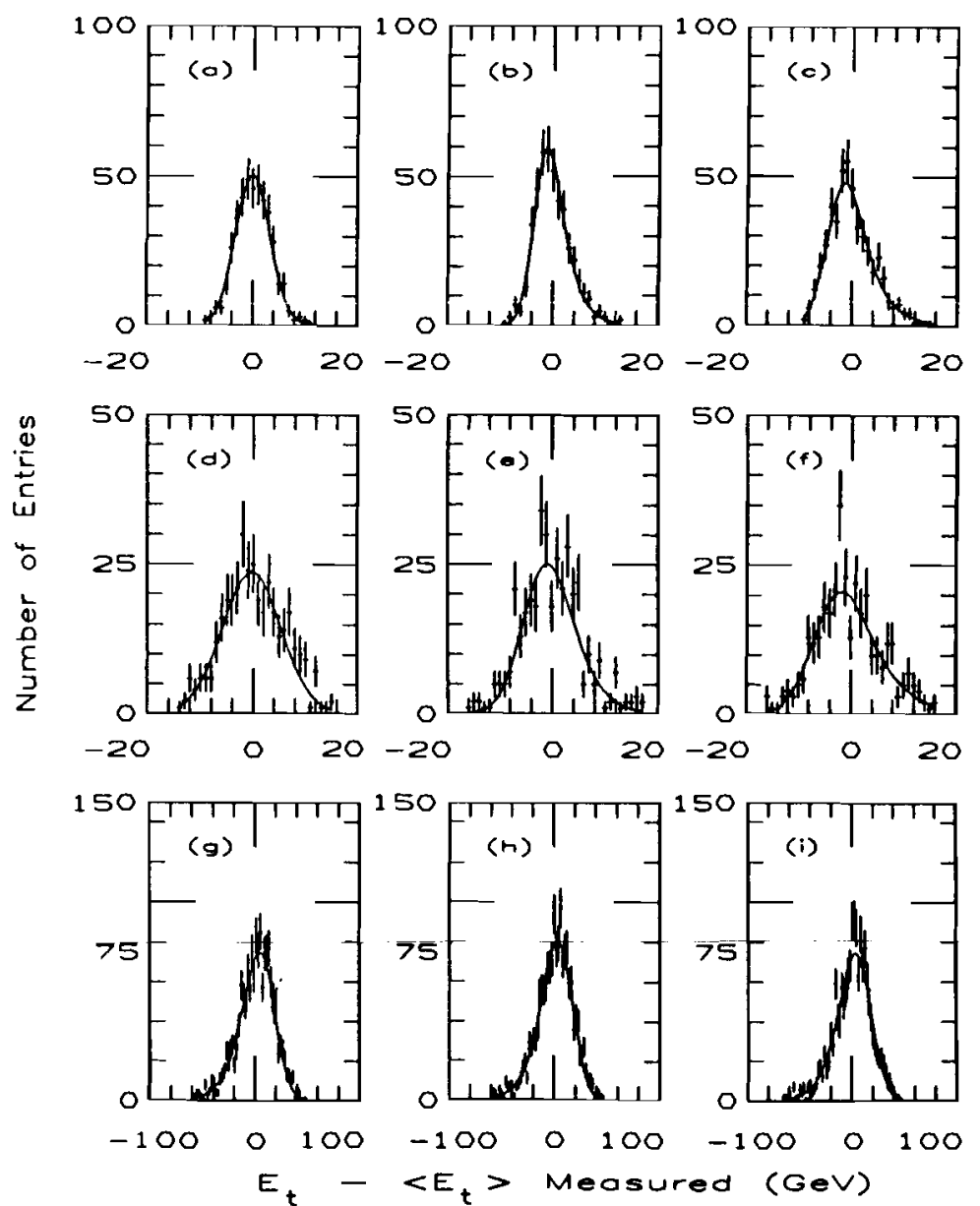

Figure 26. The simulated response of the detector at several $E_{t}$ 's for jets. The response is shown for the following cone sizes and energies: a) cone size of 0.4, b) cone size of 0.7 , and c) cone size of 1.0 with true $E_{1}$ of $20 \mathrm{GeV}$; d) cone size of $0.4, \mathrm{c}$ ) cone size of 0.7 , and f) cone size of 1.0 with true $E_{t}$ of $50(\mathrm{GeV} ; \mathrm{g}$ ) cone size of $0.4, \mathrm{~h}$ ) cone size of 0.7 , and i) cone size of 1.0 with true $E_{t}$ of $200 \mathrm{GeV}$. 
Table XII. The values of the parameters obtained for the parameterization of the Mean, for each conc size.

\begin{tabular}{cccc}
\hline Variable & Cone Size 0.4 & Cone Size 0.7 & Cone Size 1.0 \\
\hline$A$ & $1.139 \times 10^{-7}$ & $7.979 \times 10^{-8}$ & $4.679 \times 10^{-8}$ \\
$B$ & -0.358 & $-3.826 \times 10^{-2}$ & -0.299 \\
$C$ & $-1.561 \times 10^{-4}$ & $-1.089 \times 10^{-4}$ & $-6.288 \times 10^{-5}$ \\
$D$ & -1.691 & -2.070 & -1.830 \\
$E$ & 1.023 & 1.005 & 0.978 \\
$F$ & 3.485 & 4.868 & 6.080 \\
\hline
\end{tabular}

Table XIII. The values of the parameters oblained for the paraneterization of the Sigma, for each cone size.

\begin{tabular}{|c|c|c|c|}
\hline Variable & Cone Size 0.4 & Cone Size 0.7 & Cone Size 1.0 \\
\hline$W$ & 1.350 & 2.012 & 3.186 \\
\hline$x$ & 2.297 & 16.465 & 13.082 \\
\hline$y$ & 0.453 & 0.409 & 0.353 \\
\hline$Z$ & -2.668 & -6.306 & -8.316 \\
\hline
\end{tabular}

With response functions in hand, the jet $E_{t}$ and resolution corrections for uneasured jet cross section can be evaluated. Overall, the procedure is conceptually like a nuonte carlo simulation which would start with a true $E_{l}$ spectrum for jets, pass the jets througl the detector sinnuation and clustering routine and cventually yield a llicasured spectrum. The actual procedure was a quick version of the above which
Table XIV. The values of the parameters obtained for the parameterization of the Slope, for each cone size.

\begin{tabular}{cccc}
\hline Variable & Cone Size 0.4 & Cone Size 0.7 & Cone Size 1.0 \\
\hline$M$ & 0.0329 & 0.0403 & 0.00642 \\
$N$ & 1.187 & 1.171 & 3.621 \\
CUTOFF & $40 \mathrm{GeV}$ & $48 \mathrm{GeV}$ & $70 \mathrm{GeV}$ \\
$Q$ & -0.0855 & -0.0935 & -0.0823 \\
$R$ & 2.118 & 5.187 & 3.525 \\
\hline
\end{tabular}

uses the parameterized response functions rather than actually throwing events. A tunable function was used for the true spectrum and this function was tuned until the $x^{2} /$ dof between the sineared spectrum calculated by the program and the measured data spectrum was minimized. Figure 27 illustrates how this unsmearing procedure works

The procedure started with a parameterized curve that represents the true (but unknown) inclusive jet cross section. For this study the following parameterization was found to be capable of describing the cross section:

$$
\begin{aligned}
P\left(E_{l}^{\text {true }}\right) & =A\left(\left(E_{l}^{t r u c}\right)^{-M}\left(1-x_{t}+C x_{l}^{2}\right)^{N}\right), \\
x_{l} & =2 E_{l}^{\text {true }} / 1800,
\end{aligned}
$$

where $M, C$, and $N$ are the tunable parameters. $(A$, the normalization, was obtained by requiring that the area of the smeared distribution from the parameterized curve c(lual the area in the data.) This parameterization was found to fit both standard 


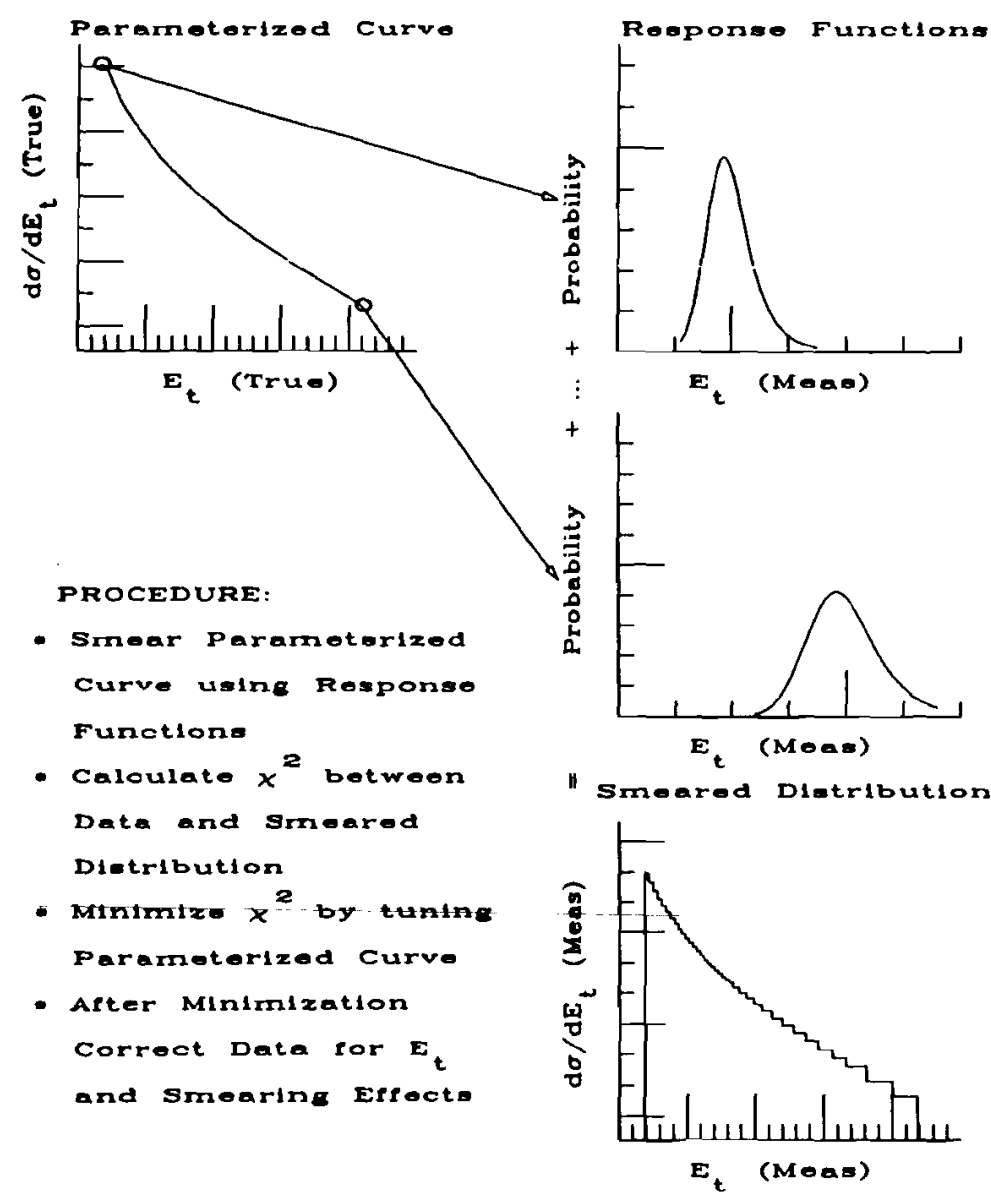

Figure 27. An illustration of the unsmearing procedure.
QCD and a range of compositeness curves very wel]. In addition, this functional form yielded a smeared distribution which agreed well with the data.

This parameterized curve was then smeared and degraded using the simulationderived response fuictions and the resulting smeared distribution was collected into the same size bins that were used for the data spectrum; in this way, effects of bin-integration were included in the comparison with the binned data. For a given measured bin in the data where the low edge of the bin is low and the high edge is high, the following convolution was performed to obtain a smeared distribution for this bin:

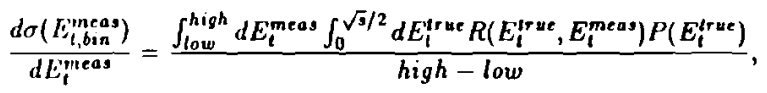

where $P\left(L_{t}^{\text {true }}\right)$ is the value of the parameterized curve evaluated at $E_{l}^{t r u e}$ and the response function $R\left(E_{l}^{\text {true }}, E_{t}^{\text {meas }}\right)$ represents the probability that a true jet at $E_{i}^{\text {frue }}$ would contribute to the nieasured spectrum at $E_{\mathfrak{l}}^{\text {meas. }} E_{i, b i n}^{\text {meas }}$ is the calculated average value of $E_{4}^{\text {meas }}$ for each bin. This integration was done from $0-\sqrt{5} / 2$ (where $\sqrt{s}$ is defined to be the center of mass energy) since this covered the range of possible jet $E_{\ell}$ 's expected to be observed. This convolution was performed for every bin used in the analysis, thus a smeared-and-binned distribution from the parameterized curve was derived.

Next, a sum of the bin-by-bin $x^{2}$ s between the data and smeared distribution was calculated using the statistical errors from the data. The procedure was then iterated modifying $M, C$, and $N$ until the $\chi^{2} /$ dof was minimized. In Figure 28 the results of the unstnearing procedure (a comparison of smeared and true spectra and 
the bin-by-bin residuals between the smeared distribution and the measured data) for cone sizes of $0.4,0.7$, and 1.0 are plotted. The values obtained for $M, C, N$, and $A$ are given in Table XV along with the $\chi^{2} / d o f$.

llaving found the best fit for the paramcterized curve which when smeared agreed with the data, the data points must be moved onto this parameterized curve. In order to do this, the following variables were defined for every bin in the data:

$$
E_{i, b i n}^{\text {meas }}=\frac{\int_{\text {low }}^{\text {high }} d E_{l}^{\text {meas }} \int_{0}^{\sqrt{s} / 2} d E_{l}^{\text {true }} E_{l}^{\text {meas }} R\left(E_{l}^{\text {true }}, E_{l}^{\text {meas }}\right) P\left(E_{t}^{\text {true }}\right)}{\int_{\text {low }}^{\text {high }} d E_{l}^{\text {meas }} \int_{0}^{\sqrt{d} / 2} d E_{l}^{\text {true }} R\left(E_{l}^{\text {true }}, E_{t}^{\text {meas }}\right) P\left(E_{l}^{\text {true }}\right)},
$$

and

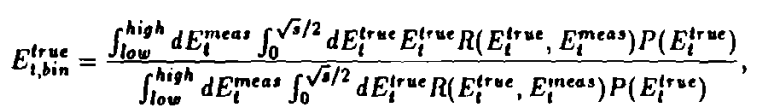

$E_{l, b i n}^{\text {tree }}$ is the calculated average value of $E_{l}^{\text {trre }}$ that the smearing procedure predicts for each bin (this value results from both resolution and energy degradation). The energy corrections to the data-points was defined in such a way that the average neasured $E_{l}$ of a given bin was scaled up to the average true $E_{l}$ contributing to that bin:

$$
E_{i, \text { bin }}=\frac{E_{i, \text { bin }}^{\text {true }}}{E_{t, b \text { in }}^{\text {meas }}} E_{i, \text { bin }}^{\text {dota }}
$$

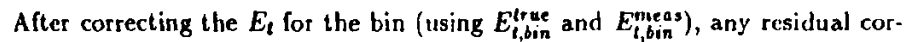
rection was performed by a scaling of cross section using the ratio of the value of the parameterized curve evaluated at $E_{f, b i n}^{t r u e}$ and the value of the smeared distrihution associated with this bin at $E_{0, b i n}^{\text {meass: }}$

$$
C S_{b \text { in }}=C S_{b i n}^{d a s a}\left(\frac{d \sigma\left(E_{l, b i c}^{\text {true }}\right)}{d E_{l}^{\text {trie }}}\right) /\left(\frac{d \sigma\left(E_{b, b i n}^{\text {meas }}\right)}{d E_{t}^{\text {meas }}}\right)
$$
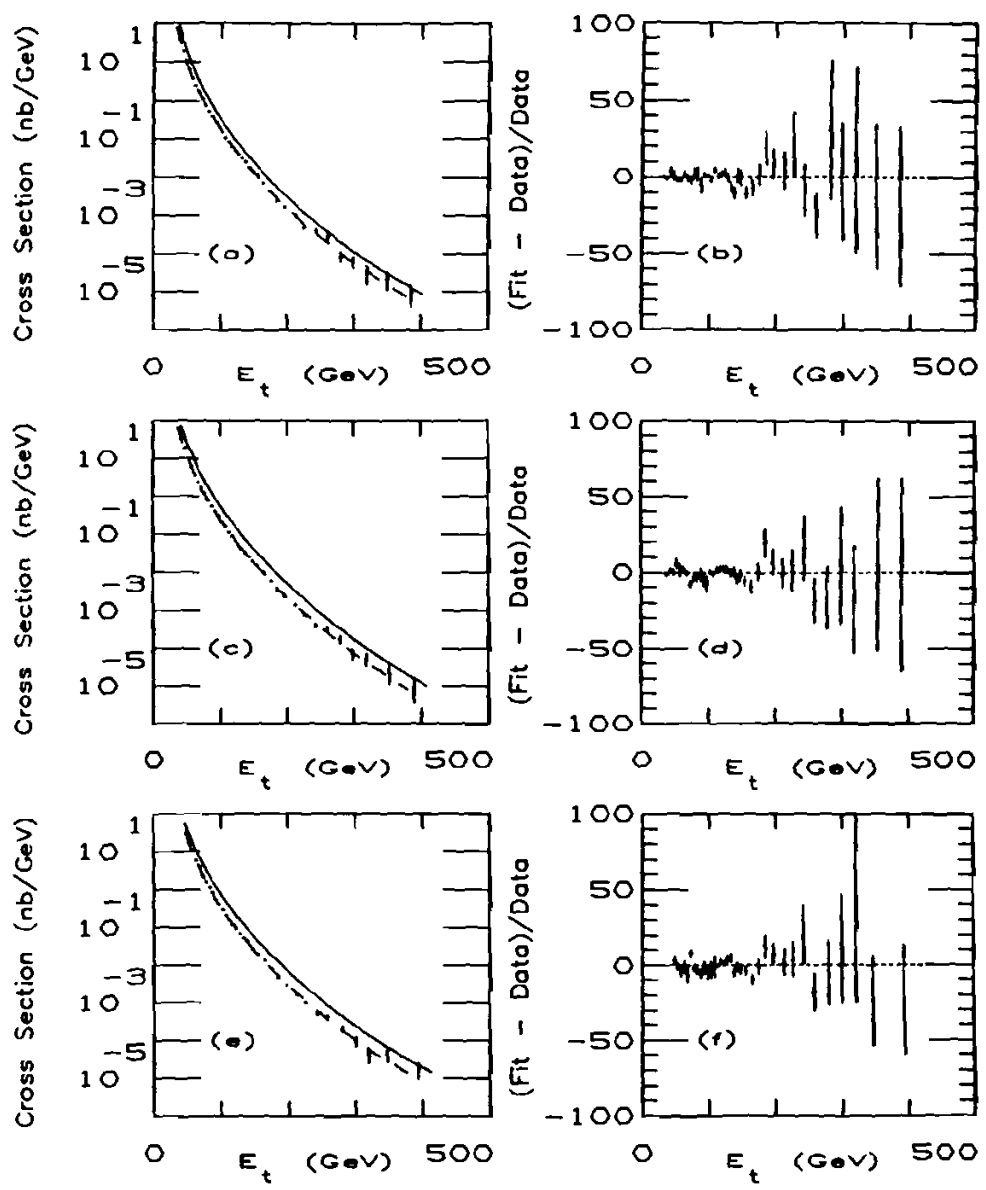

Figure 28. Unsmearing plots for cross sections of the various cone sizes. The solid line represents the unsmeared parameterized curve and the dashed line represents the smeared parameterized curve. Plotted are the a) fits and b) residuals for cone size of $0.4, c)$ fits and d) residuals for cone size of 0.7 , e) fits and f) residuals for cone size of 1.0 . 
Table XV. Fits to parameterized curve used in unsincaring procedurc.

\begin{tabular}{cccc}
\hline Parameter & Cone Size 0.4 & Cone Size 0.i & Cone Size I.0 \\
\hline$A$ & $0.11307 \times 10^{10}$ & $0.22106 \times 10^{10}$ & $0.12370 \times 10^{10}$ \\
$M$ & 4.405 & 4.431 & 4.195 \\
$C$ & 0.632 & 0.578 & 0.683 \\
$N$ & 15.867 & 15.772 & 18.102 \\
$\chi^{2} /$ dof & 1.303 & 1.220 & 1.579 \\
\hline
\end{tabular}

Thus, $E_{t, b i n}$ and $C S_{b i n}$ are the corrected $E_{t}$ and cross section for each bin in the inclusive jet cross section. Figure 29 shows the measured data and the corrected data (for a cone size of 0.7 ). As shown, the $E_{\text {t }}$ corrections move the data horizontally in this plot and the smearing corrections move the data vertically.

This procedure lias been applied to the 1988-1989 CDP jet data set for cone sizes of $0.1,0.7$, and 1.0. The corrections obtained for cone sizes of $0.4,0.7$ and 1.0 are listed in Tables XVI, XVII, and XVIII respectively. 'The results were found to be consistent with results from the $1987 \mathrm{CDF}$ inclusive jet measurement: Figure 30 shows the cross section calculated using the data collected by CDF in their 1987 data rinn (for a conc size of 0.6 ) together with the cross section calculated here using the CDF $1988-1989$ data set (with a cone size of 0.7 ). Agreement is expected only within systematic uncertainty since the assumptions about jet resolution have cvolved since 1987 ; in addition, a correction for energy loss outside the clustering cone was applied in the 1987 analysis, unlike the current analysis.

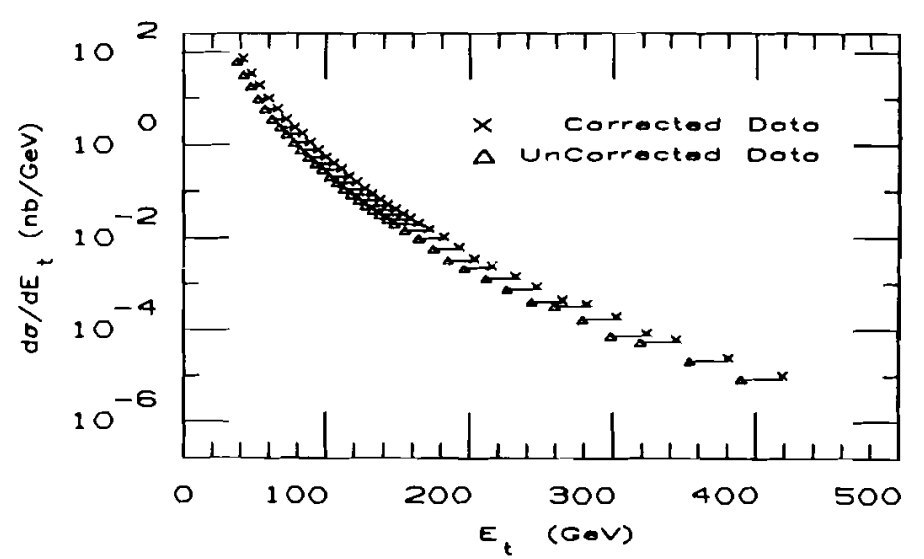

Figure 29. Unsmearrd and corrected cross section for jets with a cone size of 0.7 the lines indicate direction of corrections.

The $E_{t}$ corrections extracted from the current detector simulation are also consistent with the $E_{1}$ corrections from the 1987 analysis. Figure 31 shows the magnitude and dircction of the $E_{t}$ corrections from the 1987 resutt (72) for a cone size of 0.6 where the energy out of the cone corrections were removed, plotted along side the result arrived at using SETPR'] for a cone of 0.7 .

Dijet balancing techniques, described in Appendix A, were first introduced by the $U \Lambda 2$ collaboration $[73,74]$ and have been used extensively in jet measurements at (CDF $[75,76]$ Using these techniques, the jet resolution extracted from the sinulation was found to be consistent with what was observed in the data. Figure 32 shows $\sigma^{\prime} / \sqrt{2}$ vs $E_{t}$ for jets with a cone size of 0.7 in the data compared to the simulation where 
Table XVI. Corrections to the raw jet cross section and related quantities for a jet clustering cone size of 0.4 .

\begin{tabular}{|c|c|c|c|c|}
\hline \multirow{2}{*}{$\begin{array}{c}\text { Mean } \\
E_{l}(\mathrm{GeV}) \\
\end{array}$} & \multicolumn{4}{|c|}{ Snearing Corrections } \\
\hline & Lower Edge & Upper Edge & Mcan $E_{1}$ & Cross Section \\
\hline 32.21 & 1.124 & 1.126 & 1.125 & 1.156 \\
\hline 37.25 & 1.126 & 1.123 & 1.125 & 1.109 \\
\hline 42.28 & 1.123 & 1.126 & 1.124 & 1.103 \\
\hline 47.24 & 1.126 & 1.129 & 1.128 & 1.097 \\
\hline 52.29 & 1.129 & 1.127 & 1.129 & 1.086 \\
\hline 57.33 & 1.127 & 1.123 & 1.126 & 1.083 \\
\hline 62.30 & 1.123 & 1.119 & 1.121 & 1.084 \\
\hline 67.41 & 1.119 & 1.115 & 1.117 & 1.086 \\
\hline 72.42 & 1.115 & 1.111 & 1.113 & 1.088 \\
\hline 77.31 & 1.111 & 1.108 & 1.110 & 1.090 \\
\hline 82.37 & 1.108 & 1.105 & 1.106 & 1.093 \\
\hline 87.32 & 1.105 & 1.102 & 1.103 & 1.095 \\
\hline 92.37 & 1.102 & 1.099 & 1.100 & 1.097 \\
\hline 97.36 & 1.099 & 1.097 & 1.098 & 1.100 \\
\hline 102.32 & 1.097 & 1.094 & 1.095 & 1.102 \\
\hline 107.31 & 1.094 & 1.092 & 1.093 & 1.104 \\
\hline 112.50 & 1.092 & 1.090 & 1.091 & 1.106 \\
\hline 117.47 & 1.090 & 1.088 & 1.089 & 1.108 \\
\hline 122.43 & 1.088 & 1.086 & 1.087 & 1.110 \\
\hline 127.39 & 1.086 & 1.085 & 1.086 & 1.112 \\
\hline 132.34 & 1.085 & 1.083 & 1.084 & 1.114 \\
\hline 137.49 & 1.083 & 1.082 & 1.083 & 1.116 \\
\hline 142.34 & 1.082 & 1.080 & 1.081 & 1.118 \\
\hline
\end{tabular}

Table XV1. Continued

\begin{tabular}{ccccc}
\hline \hline \multirow{2}{*}{ Mcan } & \multicolumn{5}{c}{ Smearing Corrections } \\
$E_{t}(\mathrm{GcV})$ & lower Edge & Upper Edge & Mean $E_{t}$ & Cross Section \\
\hline 1.47 .32 & 1.080 & 1.079 & 1.080 & 1.120 \\
154.83 & 1.079 & 1.077 & 1.078 & 1.129 \\
161.57 & 1.077 & 1.075 & 1.076 & 1.132 \\
174.62 & 1.075 & 1.073 & 1.074 & 1.134 \\
181.93 & 1.073 & 1.071 & 1.072 & 1.137 \\
196.62 & 1.071 & 1.069 & 1.070 & 1.148 \\
212.77 & 1.069 & 1.067 & 1.068 & 1.150 \\
226.41 & 1.067 & 1.065 & 1.066 & 1.152 \\
243.51 & 1.065 & 1.064 & 1.065 & 1.154 \\
260.91 & 1.064 & 1.063 & 1.063 & 1.163 \\
281.68 & 1.063 & 1.062 & 1.062 & 1.162 \\
299.29 & 1.062 & 1.061 & 1.061 & 1.160 \\
319.53 & 1.061 & 1.060 & 1.061 & 1.157 \\
349.99 & 1.060 & 1.060 & 1.060 & 1.166 \\
385.92 & 1.060 & 1.061 & 1.060 & 1.175 \\
\hline \hline
\end{tabular}

$\sigma^{\prime}=\left(\sigma_{k_{t} \|}^{2}-\sigma_{k_{t_{1}}}^{2}\right)^{1 / 2}$ also plotted is what the parameterization of the response function (for a cone of 0.7 ) predicts for the single jet resolution $\left(\sigma_{r m s}=\left(\text { Slope }^{2}+\text { Sigma }^{2}\right)^{1 / 2}\right)$.

A quantitative cvaluation of the level of agrecment for the jet resolution and $E_{t}$ corrections will be discussed in the next chapter.

This method is an improvement over the previous methods used for correcting the inclusive jet cross section for two reasons. [77] First, the old scheme for corrections 
Table XVII. Corrections to the raw jet cross section and related quantities for a jet clustering cone size of 0.7 .

\begin{tabular}{|c|c|c|c|c|}
\hline \multirow{2}{*}{$\begin{array}{c}\text { Mean } \\
E_{t}(\mathrm{GeV}) \\
\end{array}$} & \multicolumn{4}{|c|}{ Sunearing Corrections } \\
\hline & Lower Edge & Upper Eilge & Mean $E_{\mathrm{t}}$ & Cross Section \\
\hline 37.25 & 1.110 & 1.125 & 1.118 & 1.194 \\
\hline 42.20 & 1.125 & 1.131 & 1.128 & 1.094 \\
\hline 47.22 & 1.131 & 1.140 & 1.131 & 1.063 \\
\hline 52.33 & 1.140 & 1.152 & 1.145 & 1.048 \\
\hline 57.33 & 1.152 & 1.159 & 1.155 & 1.030 \\
\hline 62.30 & 1.159 & 1.161 & 1.160 & 1.022 \\
\hline 67.31 & 1.161 & 1.160 & 1.161 & 1.017 \\
\hline 72.33 & 1.160 & 1.157 & 1.159 & 1.017 \\
\hline 77.31 & 1.157 & 1.153 & 1.155 & 1.020 \\
\hline 82.36 & 1.153 & 1.149 & 1.151 & 1.025 \\
\hline 87.30 & 1.149 & 1.146 & 1.148 & 1.030 \\
\hline 92.31 & 1.146 & 1.142 & 1.144 & 1.035 \\
\hline 97.36 & 1.142 & 1.139 & 1.141 & 1.040 \\
\hline 102.39 & 1.139 & 1.136 & 1.137 & 1.045 \\
\hline 107.36 & 1.136 & 1.133 & 1.134 & 1.050 \\
\hline 112.37 & 1.133 & 1.130 & 1.131 & 1.054 \\
\hline 117.41 & 1.130 & 1.127 & 1.128 & 1.059 \\
\hline 122.38 & 1.127 & 1.124 & 1.126 & 1.063 \\
\hline 127.35 & 1.124 & 1.122 & 1.123 & 1.067 \\
\hline 132.47 & 1.122 & 1.120 & 1.121 & 1.071 \\
\hline 137.41 & 1.120 & 1.118 & 1.119 & 1.075 \\
\hline 142.34 & 1.118 & 1.115 & 1.117 & 1.079 \\
\hline 147.37 & 1.115 & 1.113 & 1.114 & 1.083 \\
\hline
\end{tabular}

Table XIII. Continued

\begin{tabular}{|c|c|c|c|c|}
\hline \multirow{2}{*}{$\begin{array}{c}\text { Mean } \\
E_{t}(\mathrm{GeV})\end{array}$} & \multicolumn{4}{|c|}{ Sinearing Corrections } \\
\hline & Lower Edge & Upper Edge & Mean $E_{\mathrm{t}}$ & Cross Section \\
\hline 154.62 & 1.113 & 1.110 & 1.112 & 1.094 \\
\hline 164.42 & 1.110 & 1.106 & 1.108 & 1.101 \\
\hline 174.70 & i.iūó & 1.103 & 1.105 & 1.107 \\
\hline 184.64 & 1.103 & 1.101 & 1.102 & 1.113 \\
\hline 196.34 & 1.101 & 1.097 & 1.099 & 1.127 \\
\hline 211.73 & 1.097 & 1.093 & 1.095 & 1.135 \\
\hline 226.08 & 1.093 & 1.090 & 1.092 & 1.141 \\
\hline 243.19 & 1.090 & 1.088 & 1.089 & 1.147 \\
\hline 259.56 & 1.088 & 1.085 & 1.086 & 1.161 \\
\hline 278.95 & 1.085 & 1.082 & 1.083 & 1.166 \\
\hline 298.70 & 1.082 & 1.080 & 1.081 & 1.170 \\
\hline 318.75 & 1.080 & 1.078 & 1.079 & 1.172 \\
\hline 353.57 & 1.078 & 1.076 & 1.077 & 1.189 \\
\hline 389.56 & 1.076 & 1.073 & 1.074 & 1.224 \\
\hline
\end{tabular}

did not account for bin-integration effects having to do with the size of the bin used, whereas the new procedure does. The second improvement is conceptual: the actual detector response to jets is now modeled by combining the resolution effects with the $E_{1}$ corrections, whereas before the $E_{1}$ corrections where handled separately from the resolution. The reason this is more indicative of the actual physics processes involved in measuring the data is that physically a jet of $E_{f}^{\text {true }}$ is not always measured as a 
Table XV11I. Corrections to the raw jet cross section and related quantities for a jet clustering cone size of 1.0 .

\begin{tabular}{|c|c|c|c|c|}
\hline \multirow{2}{*}{$\begin{array}{c}\text { Mean } \\
E_{1}(\mathrm{GeV}) \\
\end{array}$} & \multicolumn{4}{|c|}{ Smearing Corrections } \\
\hline & Lower Edge & Upper Edge & Mean $E_{l}$ & Cross Section \\
\hline 47.30 & 0.932 & 1.006 & 0.968 & 1.817 \\
\hline 52.18 & 1.006 & 1.055 & 1.030 & 1.492 \\
\hline 57.30 & 1.055 & 1.084 & 1.070 & 1.294 \\
\hline 62.31 & 1.084 & 1.100 & 1.093 & 1.169 \\
\hline 67.51 & 1.100 & 1.108 & 1.101 & 1.111 \\
\hline 72.33 & 1.108 & 1.116 & 1.112 & 1.096 \\
\hline 77.24 & 1.116 & 1.124 & 1.120 & 1.088 \\
\hline 82.39 & 1.124 & 1.128 & 1.126 & 1.076 \\
\hline 87.41 & 1.128 & 1.130 & 1.129 & 1.067 \\
\hline 92.33 & 1.130 & 1.129 & 1.130 & 1.063 \\
\hline 97.39 & 1.129 & 1.128 & 1.129 & 1.063 \\
\hline 102.45 & 1.128 & 1.126 & 1.127 & 1.065 \\
\hline 107.46 & 1.126 & 1.124 & 1.125 & 1.068 \\
\hline 112.40 & 1.124 & 1.122 & 1.123 & 1.072 \\
\hline 117.40 & 1.122 & 1.120 & 1.121 & 1.075 \\
\hline 122.42 & 1.120 & 1.119 & 1.119 & 1.078 \\
\hline 127.37 & 1.119 & 1.117 & 1.118 & 1.082 \\
\hline 132.42 & 1.117 & 1.115 & 1.116 & 1.085 \\
\hline 137.39 & 1.115 & 1.114 & 1.111 & 1.088 \\
\hline 142.43 & 1.114 & 1.112 & 1.113 & 1.091 \\
\hline 147.46 & 1.112 & 1.111 & 1.112 & 1.094 \\
\hline 154.78 & 1.111 & 1.108 & 1.110 & 1.104 \\
\hline 164.54 & 1.108 & 1.106 & 1.107 & 1.108 \\
\hline
\end{tabular}

'lable XVIII. Continued

\begin{tabular}{|c|c|c|c|c|}
\hline \multirow{2}{*}{$\begin{array}{c}\text { Mcall } \\
E_{i}(\mathrm{GeV})\end{array}$} & \multicolumn{4}{|c|}{ Snearing Corrections } \\
\hline & Lower Edge & Upper Edge & Mean $E_{t}$ & Cross Section \\
\hline 171.76 & 1.106 & 1.104 & 1.105 & 1.112 \\
\hline 181.50 & 1.104 & 1.102 & 1.103 & 1.116 \\
\hline 196.69 & 1.102 & 1.099 & 1.100 & 1.128 \\
\hline 212.30 & 1.099 & 1.097 & 1.098 & 1.131 \\
\hline 225.88 & 1.097 & 1.094 & 1.096 & 1.134 \\
\hline 241.99 & 1.094 & 1.093 & 1.094 & 1.135 \\
\hline 258.34 & 1.093 & 1.091 & 1.092 & 1.143 \\
\hline 280.24 & 1.091 & 1.089 & 1.090 & 1.141 \\
\hline 300.00 & 1.089 & 1.088 & 1.088 & 1.137 \\
\hline 320.63 & 1.088 & 1.087 & 1.087 & 1.131 \\
\hline 347.38 & 1.087 & 1.086 & 1.087 & 1.133 \\
\hline 393.87 & 1.086 & 1.087 & 1.086 & 1.137 \\
\hline
\end{tabular}

jet of some average $E_{l}^{\text {meas; }}$ its relative contribution to $E_{l}^{\text {meas }}$ depends on the jet $E_{t}$ spectrum and the size of the $E_{t}^{\text {meas }}$ bin. 


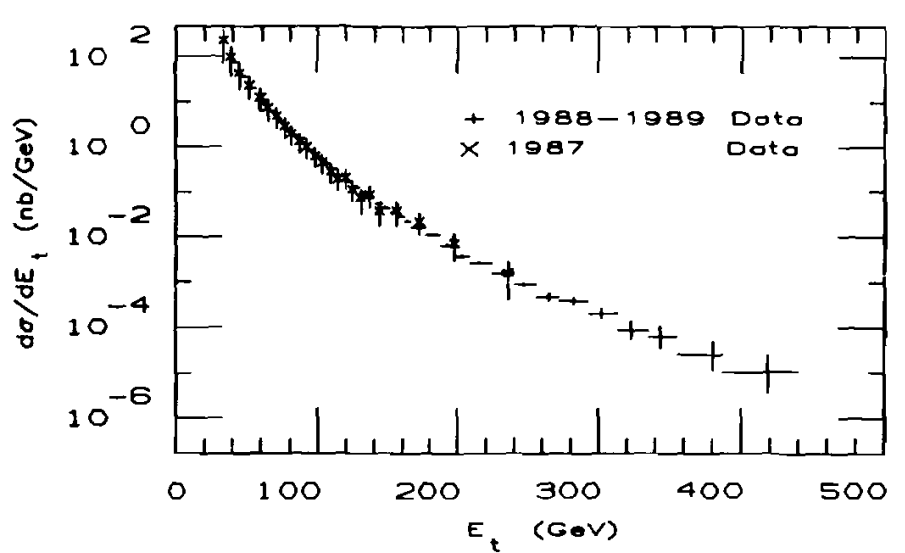

Figure 30. The 1987 cross section for jets with a cone size of 0.6 compared to 1988 1989 cross section for jets with a cone size of 0.7 .

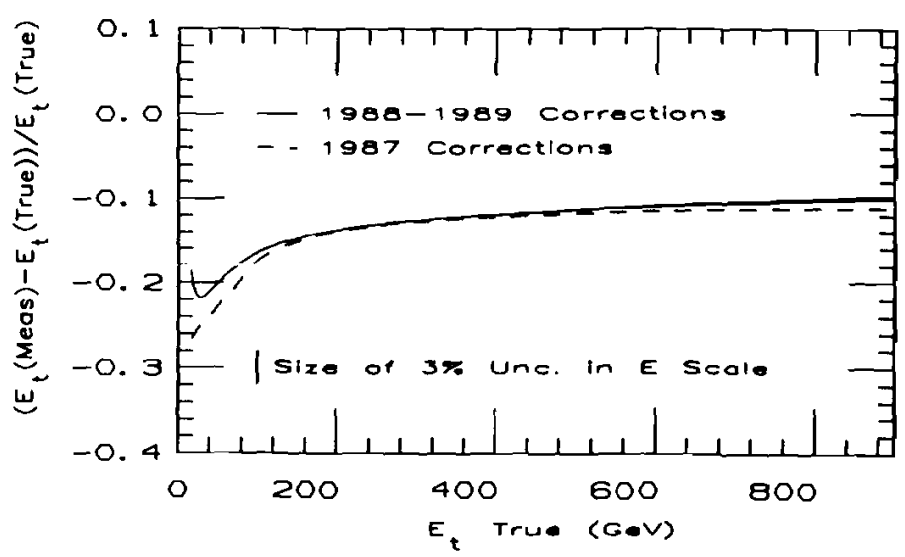

Figure 31. The $1987 E_{t}$ corrections compared to the 1988-1989 $E_{t}$ corrections. 


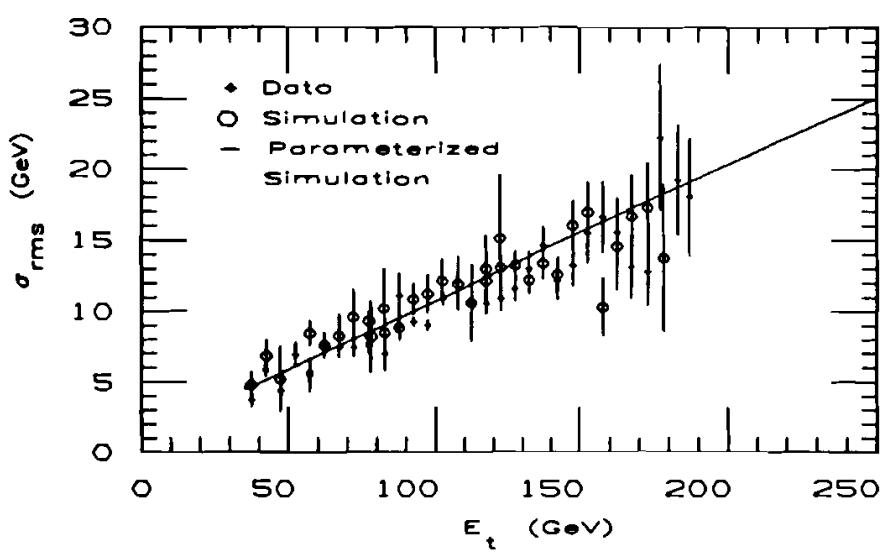

Figure 32. Comparison of the rins resolution for data, simulation and the parame terized response for jets with a cone size of 0.7 . 


\section{CHAPTER V}

\section{SYSTEMATIC UNCERTAINTIES}

Systematic uncertainties in tle jet $E_{1}$ scalc and resolution introduce a systematic uncertainty into the measurement of the inclusive jet cross section. The systematic uncertainty on the luminosity also contrilutes to the unrertainty on the cross section. This chapter will describe the uncertainty on the jet $E_{\ell}$ scale, jet resolution, and luminosity. It will then explain how these uncertainties are factored into the inclusive jet cross section.

\section{A. E Scale Systematic Uncertainties}

The jet $E_{t}$ scale was determined using a detector simulation which was tuned to reproduce the single pion response and the jet fragmentation using both test beam and collider data. $[65,67]$ The major source of systematic uncertainty on the jet $E_{\mathrm{t}}$ scale is thic calorimeter response uncertainty in the azimuthal boundary (cracks) regions of the detector. Other sources of uncertainty on the jet $E_{\mathrm{t}}$ scale include the calorimeter respense in other areas of the detector, the fraginentation tuning in the simulation, and the energy from the underlying event (within the clustering cone). The energy from the underlying event is energy associated with the interactions from spectator partons and sinall collisions occurring without any hard scattering (mininium bias events). An uncertainty is also associated with the jet $E_{t}$ scale based on preliminary results from the andysis of the 1990 test bean data where a difference in the calorimeter response to pions has becn observed.

\section{Calorimeter Response}

Pions in an energy range from $750 \mathrm{MeV}$ to $20 \mathrm{GeV}$ were extracted from minimum bias collider data, and pions at higher energies of $57 \mathrm{GeV}$ and $145 \mathrm{GeV}$ were used from the 1984/1985 test beam data to measure the single pion response in the central calorimeter. The response of the calorincter to pions was measured using both the iracking charuber and calorimeter. Neutral pions $\left(\pi^{0}\right.$ 's) cannot be measured in the tracking chamber, but do leave energy in the calorineter. The $\pi^{01}$ s that overlap with charged pions can cause the response of the calorineter towers to pions to be tnismeasum. In order to estimate this background, it was assumed that the shower leakage from the target tower was small and that the energy found in neighboring towers was from $\pi^{0}$ background. The background was estimated using the energy found in the neighboring electromagnetic calorimeter towers.

Shower leakage from the target tower and the possibility of finding more $\pi^{0} \mathrm{~s}$ in the target tower than in the neighboring towers are possible sources of mismea. surement for this background. The average border tower energy was small $(\sim 2 \%$ of the inomentum), therefore a conservative $5 \%$ systematic uncertainty was assigned to the $\pi^{0}$ background subtraction in the single pion response. [65] This is the dominant uncertainty for pions with cnergies less than $25 \mathrm{GeV}$.

The azimuthal crack response observed in the data is not in agreement with the response used in the simulation to obtain the energy and smearing corrections. The size of the shift in the $E_{t}$ scale calculated using the simulation with and without the azimuthal crack response is a $7 \%$ shift in the measured jet $E_{t}$. This shift is a measure of the unccrtainty in the azimuthal crack responsc on the $E_{\imath}$ scale. Therefore, a 7\% 
uncertainty on the measured jet $E_{t}$ was calculated for the azimuthal crack uncertainty using the simulation.

The response in the azimuthal crack region was also studied using pions in the test beam. There was difficulty in determining the amount of muon contamination in the pion beam which varied run by run. Run by run variations in the measured response and the uncertainty in the muon contannination, as well as tower to tower variations in the response and an uncertainty in the tower calibration, gave a total uncertainty of $8 \%$ on the crack response. [78] This was the dominant source of uncertainty in the response for pions with energies greater than $25 \mathrm{GeV}$. This $8 \%$ uncertainty on the response is in addition to the $7 \%$ unccrtainty on the measured jet $E_{\text {. }}$

\section{Fragmentation Tuning}

The non-linearity of the central hadron calorimeter to clarged particles is a major contribution to the energy loss of the calorineter. Jet fragmentation is a measure of the distributions of the charged particles found in the central tracking chamber associated with jets and therefore effects how well the simulation reproduces the jet energy corrections. The overall tuning of the fragmentation in the simulation is in good agreement with the data. The uncertainty in the fragmentation tuning, however, is correlated with the efficiency of finding tracks in jets. The uncertainty in this tracking efficiency therefore affects the uncertainty on the fragmentation tuning. The uncertainty in the efficiency of finding tracks in jets was found to be $\pm 7 \%$, based on the trends observed in measuring the efficiency and the correctness of the embedding scheme.
Since the efficiency of finding tracks in jets is related to the ability of the detector to resolve trarks iu a dense track environment, the tracking efficiency was paranneterized in terms of the distance of closest approach between two charged tracks in the central tracking chamber. The elficiency was deternined by injecting charged tracks from Monte Carlo into jet events, and the determining the reconstruction efficiency, and parameterizing this in terms of the average separation to the nearest track in the jet. In this procedure, there is an inherent uncertainty based on whether the efficiency measured in such a way is to be applied to one or two tracks. This uncertainty lt was Jecided to apply the efficiency to both tracks per pair and as a systematic uncertainty on this ineasurement to use the case of applying the efficiency to one track per pair.

The limited statistics in the data at high $E_{\mathfrak{t}}$ meant this efficiency could not be determined for jets with $E_{\ell}>250 \mathrm{GcV}$. A limit of $400 \mathrm{GcV}$ was arbitrarily chosen as the $E_{t}$ at which to pin the efficiency such that above this limit the efficiency does not change. This limit was changed to $250 \mathrm{GeV}$, a point where the statistics were reasonable in the data, in order to clieck the uncertainty on this quantity.[67]

3. Underlying Event

The underlying event $E_{t}$ density was measured using dijet events in the 1987 data; that neasurement was used here.[79] Cuts used to define the dijet sample, such as the maximum allowed $E_{t}$ for any third jet in the event and the azinuthal separation requirements for the jets, give a range of underlying event $E_{t}$ densities. A systematic uncertainty of $\pm 300 \mathrm{McV} / \mathrm{rad}^{2}$ refiects the uncertainty on the $E_{t}$ density due to the choice of appropriate dijet cuts. 
Run by run variations in the detector performance were another source of uncertainty in this measurement. These run by run variations gave a systematic uncertainty of $\pm 100 \mathrm{MeV} / \mathrm{rad}^{2}$. An additional $80 \mathrm{MeV} / \mathrm{rad}^{2}$ statistical uncertainty was also as. sociated with this measurement. Finally, the $E_{\ell}$ density was determined and found to be independent of jet $E_{l}$ within $\pm 100 \mathrm{MeV} / \mathrm{rad}^{2}$. Together with the other uncer tainties, the total uncertainty on the underlying event $E_{t}$ density was determined to be $\pm 340 \mathrm{McV} / \mathrm{rad}^{2}$.

4. 1990 Test Beam Results

Recent results from the $1990 \mathrm{CDF}$ test beam have led to a difference in the calorimeter response of the detector to $150 \mathrm{GcV}$ pions and the anount of shower leakage from the target tower. Compared to 1985 test beam data, the calorimeter response to $150 \mathrm{GeV}$ pions was found to be $6 \%$ lower in the 1990 test beam data. In addition to this change in response, shower leakage from the target tower contributing encrgy to the neighloring towers was observed in the CEM. This effects the initial assumption that shower feakāge froin the target tower was small and that all the energy found in neigliboring towers was from $\pi^{0}$ background. A study is currently in progress of 1990 test beam data, which also includes exaınining the calorimeter response to pions at higher energies which may have some effect on this measurernent. While analysis of the 1990 test beam data is still under study, these early results nced to be examined here to decide if the size of these effects on the jet $E_{l}$ scale are large enough to warrant further investigation.
5. Resulting Jet $E_{t}$ Scale Uncertainty

l'or the calorimeter and fragmentation, the resulting uncertainty on the jet $E_{t}$ scale was determined using a loss plot. A loss plot is a incasure of the average energy lost due to the hadronic non-linearity, which is directly related to the size of the jet corrections. More specifically, it is the sum of the difference in the momentum and the average calorimeter response $(<R(p)>)$ of the tracks in the cone of the jet over the measured $E_{t}$ of the jet, as defined in the following equation,

$$
\text { Loss }=\frac{\sum_{i=1}^{\# \text { tracks }}\left(<R(p)>-p_{i, j}\right)}{E_{t, j}} .
$$

The difference in the average calorimeter response and the momentum of a track is the amount of energy loss by the track in the calorimeter. Summing this difference over all the tracks in a jet gives the amount of energy loss by the jet. Dividing this sum by the measured jet $E_{1}$ gives the fraction of energy loss by the jet. The difference between the loss plot measured in the data and the simulation (which can be varied to reflect various systematic uncertainties), is a measure of the uncertainty in the jet $E_{\ell}$ scale.

To find the effect of the calorimeter uncertainty on the jet $E_{t}$ scale, the average response of the calorimeter used in making the loss plot was varied in the data by the uncertainty on the single pion response. The resulting loss plots for these uncertainties were then fit to the function:

$$
f=\frac{N}{E_{i}^{A}} e^{-B E_{i}}
$$


where $E_{1}$ is measured jet $E_{1}$. The loss plots extracted from the data using the measured calorimeter response, as well as the loss plots obtained by changing the response within it's uncertainties which were mentioned earlier, can be seen in Figure 33. The difference in the fits obtained for these plots multiplied by the measured jet $E_{t}$ and divided by the corrected jet $E_{t}$ gives the fractional uncertainty on the corrected (or true) jet $E_{l}$ scalc. The resulting uncertainty on the jet $E_{t}$ scale for each cone size is listed in Table XIX. This uncertainty includes all the uncertainties on the calorime ter response mentioned earlier except for the $7 \%$ uncertainty on the measured jet $E_{t}$ scale due to the difference in the jet $E_{1}$ scalc calculated using the simulation with and without the azimuthal crack response.

Using this same technique, the fragmentation uncertaintics werc varied in the simulation and loss plots were extracted for cach of the different uncertainties, as well as the tuned value (sec Figure 34). The uncertainty for each of these effects on the jct $E_{\imath}$ scale is listed in Table XX for each cone size.

The uncertainty on the jet $E_{t}$ scale due to the uncertainty on the underlying event $E_{\ell}$ density is the uncertainty on the $E_{t}$ density times the area of the jet clustering cone. The area of the jet clustering cone is basically $\pi R^{2}$, where $R$ is the cone size used in the clustering algorithm. For cone sizes of $0.4,0.7$, and 1.0 the uncertainty in the jet $E_{1}$ scale is $171 \mathrm{MeV}, 523 \mathrm{MeV}$, and $1068 \mathrm{McV}$ respectively. This uncertainty is independent of jet $E_{l}$, thus the fractional uncertainty on this number grows smaller with increasing jet $E_{\mathrm{t}}$. Table XXI lists this uncertainty as a fraction of the corrected jet $E_{t}$
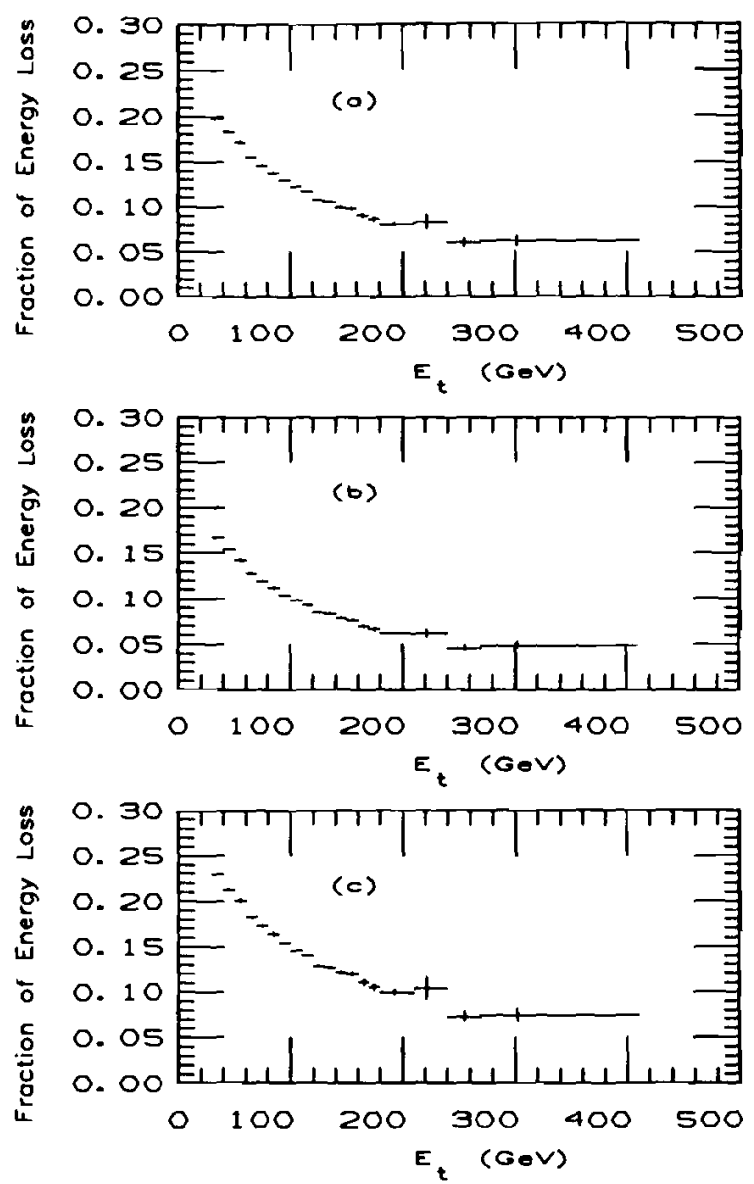

Figure 33. Loss plots as a function of measured jet $E_{t}$ for the data. Using a) the . the response, c) the lower limit of the uncertainty on the response. 
Table XIX. Calorimeter response fractional systematic uncertainty on the corrected jet $E_{\mathrm{t}}$ scalc.

\begin{tabular}{cccc}
\hline \hline True Jet & \multicolumn{3}{c}{ Calorimeter Response } \\
$E_{l}(\mathrm{GeV})$ & Cone Size 0.4 & Cone Size 0.7 & Cone Size 1.0 \\
\hline 20.0 & 0.0265 & 0.0258 & 0.0271 \\
40.0 & 0.0248 & 0.0247 & 0.0252 \\
60.0 & 0.0233 & 0.0238 & 0.0241 \\
80.0 & 0.0220 & 0.0228 & 0.0231 \\
100.0 & 0.0208 & 0.0218 & 0.0221 \\
120.0 & 0.0197 & 0.0208 & 0.0212 \\
140.0 & 0.0186 & 0.0198 & 0.0203 \\
\hline \hline
\end{tabular}

In order to examine the size of the effects seen in the 1990 test beam, the calorimeter response in the simulation was changed to reflect the new test beam results. A modificd loss plot was made based on generated particle response (which is the calorimeter response of particles generated which does not include the effect of the tracking efficiency in the simulation). The difference in the loss plots made of the generated particle response using the previous response, $\langle R(p)\rangle$ (based on 1985 test beam results) and the generated particle response using the modified response, $<R_{m}(p)>$ (based on 1990 test beam results), is a measure of the size of this difference. Figure 35 is a plot of this difference in the fractional energy loss for these generated particle responses. This result was fit to a second order polynomial in order to parameterize this difference. Table XXII lists the results of this uncertainty as
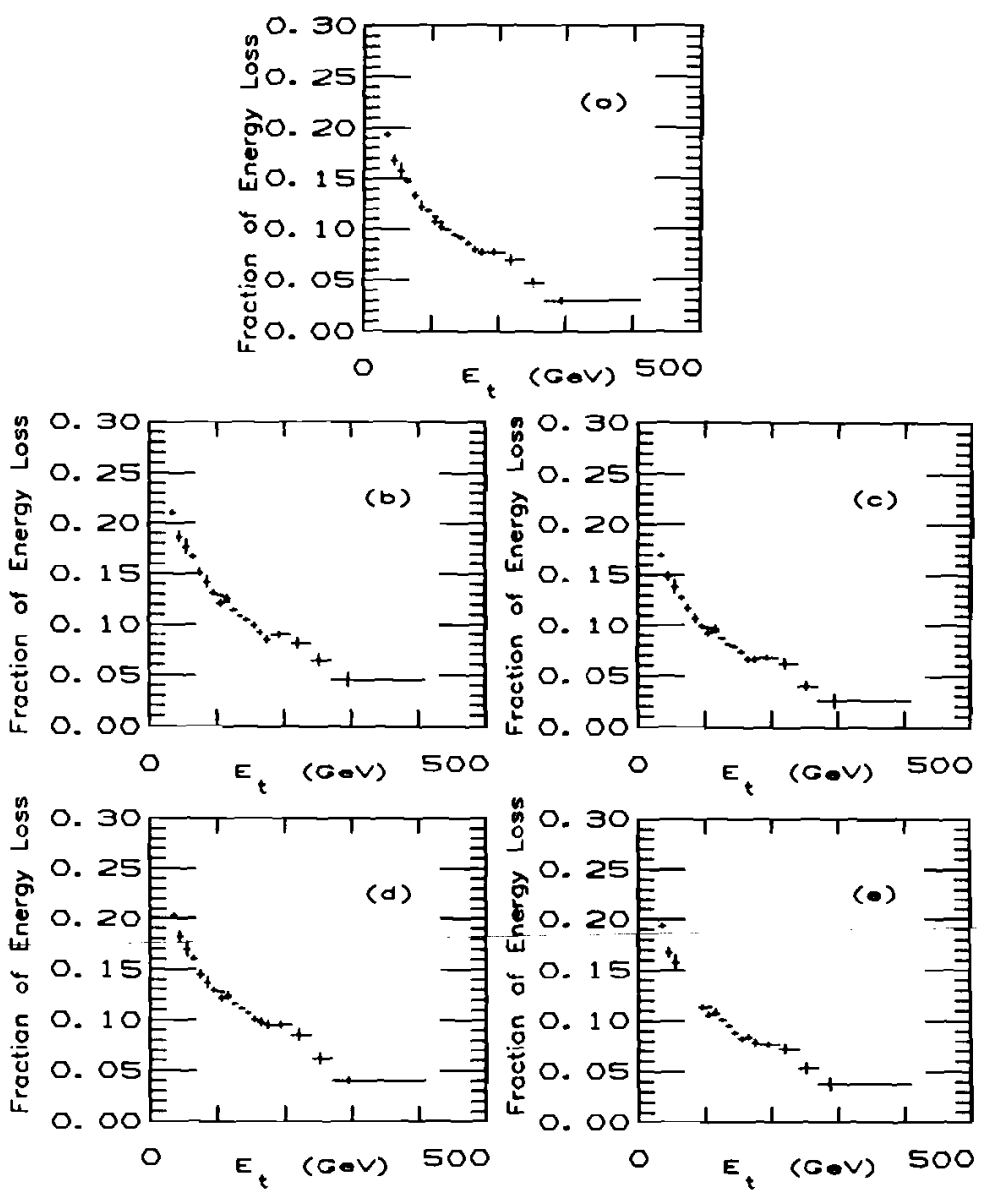

Figure 34. Loss plots from the simulation as a function of measured jet $E_{t}$. For, a) the tuned fragmentation, changing the tracking efficiency by b) $+7 \%, c)$ $-7 \%$, d) applying the efficiency to one track per pair instead of two, and e) pinning the efficiency at $250 \mathrm{GeV}$ instead of $400 \mathrm{GeV}$. 
Table XX. Fragmentation fractional systematic uncertainties on the corrected jct $E_{1}$ scale.

\begin{tabular}{ccccc}
\hline \hline True Jet & $\begin{array}{c}\text { Fragmentation } \\
E_{l}(\mathrm{GeV})\end{array}$ & $\begin{array}{c} \pm \% \\
\text { Tuning }\end{array}$ & $\begin{array}{c}\text { 1-Track } \\
\text { on Efficiency }\end{array}$ & $\begin{array}{l}\text { IIigh Pin } \\
\text { per pair }\end{array}$ \\
$250 \mathrm{GeV}$ \\
\hline 20.0 & 0.0257 & 0.0188 & -0.0131 & 0.0008 \\
40.0 & 0.0184 & 0.0157 & -0.0101 & 0.0003 \\
60.0 & 0.0148 & 0.0142 & -0.0100 & 0.0002 \\
80.0 & 0.0123 & 0.0132 & -0.0107 & 0.0001 \\
100.0 & 0.0105 & 0.0125 & -0.0115 & 0.0000 \\
120.0 & 0.0091 & 0.0120 & -0.0125 & 0.0000 \\
140.0 & 0.0080 & 0.0115 & -0.0134 & 0.0000 \\
\hline \multicolumn{5}{c}{ Cone Size 0.4 } \\
\hline 20.0 & 0.0063 & 0.0186 & -0.0017 & -0.0085 \\
40.0 & 0.0061 & 0.0172 & -0.0071 & -0.0012 \\
60.0 & 0.0065 & 0.0162 & -0.0097 & 0.0009 \\
80.0 & 0.0071 & 0.0152 & -0.0113 & 0.0013 \\
100.0 & 0.0076 & 0.0142 & -0.0123 & 0.0011 \\
120.0 & 0.0080 & 0.0133 & -0.0129 & 0.0006 \\
140.0 & 0.0084 & 0.0125 & -0.0133 & -0.0001
\end{tabular}

Table XX. Continued

\begin{tabular}{ccccc}
\hline \hline $\begin{array}{c}\text { True Jet } \\
E_{t}(\mathrm{GicV})\end{array}$ & $\begin{array}{c}\text { Fragmentation } \\
\text { Tuning }\end{array}$ & $\begin{array}{c} \pm 7 \% \\
\text { on Efficiency }\end{array}$ & $\begin{array}{c}\text { 1-Track } \\
\text { per pair }\end{array}$ & $\begin{array}{c}\text { lligh Pin } \\
250 \mathrm{GeV}\end{array}$ \\
\hline \multicolumn{5}{c}{ Cone Size 1.0 } \\
\hline 40.0 & -0.0092 & 0.0156 & -0.0024 & -0.0004 \\
40.0 & 0.0020 & 0.0165 & -0.0071 & 0.0000 \\
60.0 & 0.0062 & 0.0162 & -0.0095 & 0.0001 \\
80.0 & 0.0082 & 0.0156 & -0.0110 & 0.0001 \\
100.0 & 0.0092 & 0.0148 & -0.0120 & 0.0001 \\
120.0 & 0.0095 & 0.0140 & -0.0128 & 0.0000 \\
140.0 & 0.0095 & 0.0131 & -0.0133 & 0.0000 \\
\hline \hline
\end{tabular}


Table XXI. Underlying event $E_{t}$ density fractional systematic uncertainty on the corrected jet $E_{\ell}$ scale.

\begin{tabular}{cccc}
\hline $\begin{array}{c}\text { True Jet } \\
E_{1}(\mathrm{GeV})\end{array}$ & Cone Size 0.4 & $\begin{array}{c}E_{t} \text { Density } \\
\text { Conc Sizc 0.7 }\end{array}$ & Cone Size 1.0 \\
\hline 20.0 & 0.0085 & 0.0262 & 0.0534 \\
40.0 & 0.0043 & 0.0131 & 0.0267 \\
60.0 & 0.0028 & 0.0087 & 0.0178 \\
80.0 & 0.0021 & 0.0065 & 0.0134 \\
100.0 & 0.0017 & 0.0052 & 0.0107 \\
120.0 & 0.0014 & 0.0014 & 0.0089 \\
140.0 & 0.0012 & 0.0037 & 0.0076 \\
\hline
\end{tabular}

a fraction of corrected jet $E_{\ell}$. The size of this effect is smaller than the $7 \%$ uncertainty

on the measured jet $E_{t}$ scale resulting from the uncertainty in the azimuthal boundary response. Since the source of this difference is still under study, this uncertainty will be combined with the other uncertainties.

The various uncertainties were then adued in quadrature to obtain the total systematic uncertainty on the jet $E_{t}$ scale. The total systematic uncertainly is listed in Table XXIII. Figure 36 shows the breakdown of the various components of the total uncertainty on the corrected jet $E_{\mathrm{l}}$ scale as a fraction of corrected jet $E_{\mathrm{l}}$ and as a function of the corrected jet $E_{1}$.

An additional calibration uncertainty in the central electromagnetic calorineter was noted during the 1988-1989 collider run, therefore another uncertainty on the
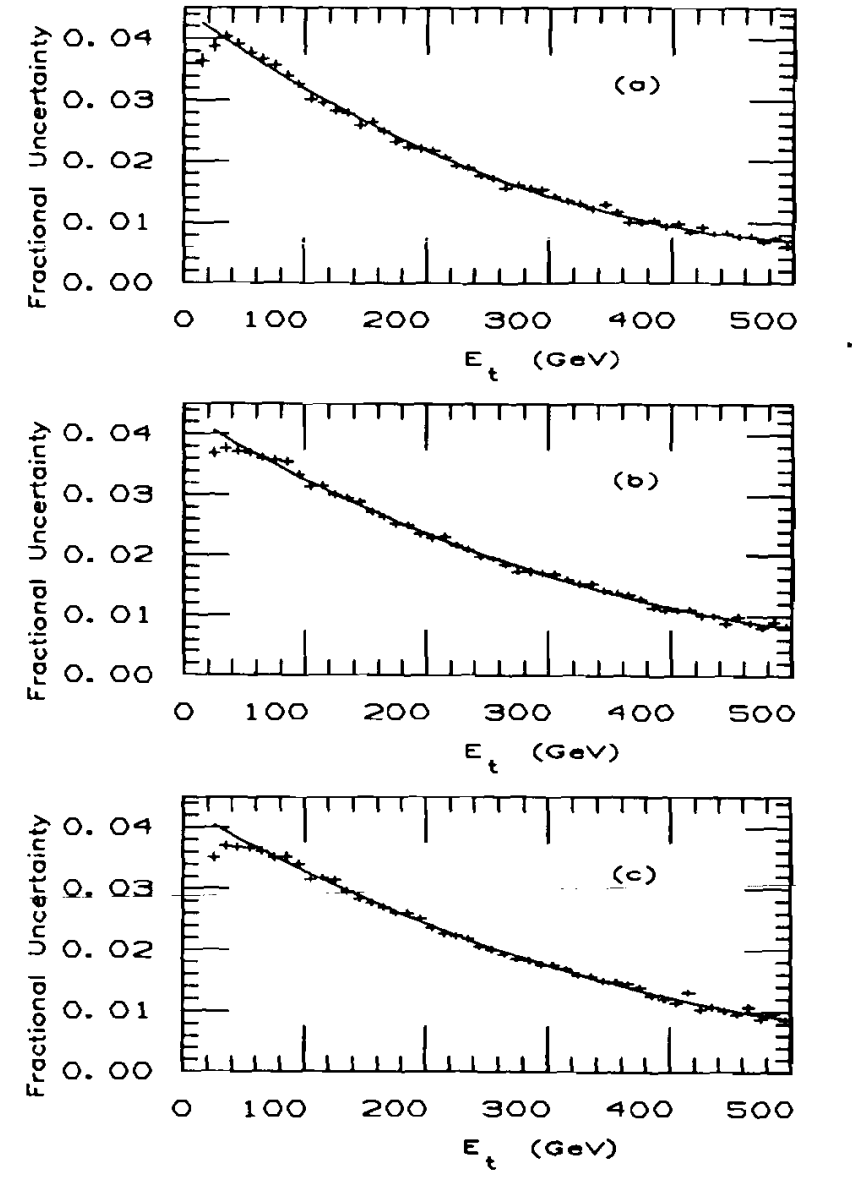

ligure 35. The fractional uncertainty of the corrected jet $E_{t}$ scale due to the difference in the 1990 test beam with previous test beam results. For a) cone size of $0.4, b$ ) cone size of 0.7 , and c) cone size of 1.0 
Table XXII. Fractional uncertainty associated with the difference in the 1990 test beam results and previous lest beam results.

\begin{tabular}{cccc}
\hline \hline True Jet & \multicolumn{3}{c}{ Test Beam Uncertainty } \\
$E_{\ell}(\mathrm{GeV})$ & Cone Size 0.4 & Cone Size 0.7 & Cone Size 1.0 \\
\hline 20.0 & 0.0419 & 0.0411 & 0.0409 \\
40.0 & 0.0392 & 0.0388 & 0.0388 \\
60.0 & 0.0367 & 0.0367 & 0.0367 \\
80.0 & 0.0342 & 0.0346 & 0.0348 \\
100.0 & 0.0319 & 0.0325 & 0.0328 \\
120.0 & 0.0297 & 0.0306 & 0.0310 \\
140.0 & 0.0275 & 0.0287 & 0.0292 \\
\hline \hline
\end{tabular}

Table XXIII. Total fractional systematic uncertainty on the corrected jet $E_{l}$ scale for each cone size.

\begin{tabular}{cccc}
\hline \hline True Jet & \multicolumn{3}{c}{ Total Systematic } \\
$E_{\text {\& }}(\mathrm{GeV})$ & Cone Size 0.4 & Cone Size 0.7 & Cone Size 1.0 \\
\hline 20.0 & 0.0812 & 0.0804 & 0.0947 \\
40.0 & 0.0777 & 0.0758 & 0.0801 \\
60.0 & 0.0761 & 0.0748 & 0.0770 \\
80.0 & 0.0749 & 0.0741 & 0.0756 \\
100.0 & 0.0739 & 0.0734 & 0.0745 \\
120.0 & 0.0730 & 0.0726 & 0.0736 \\
140.0 & 0.0722 & 0.0719 & 0.0727 \\
\hline \hline
\end{tabular}
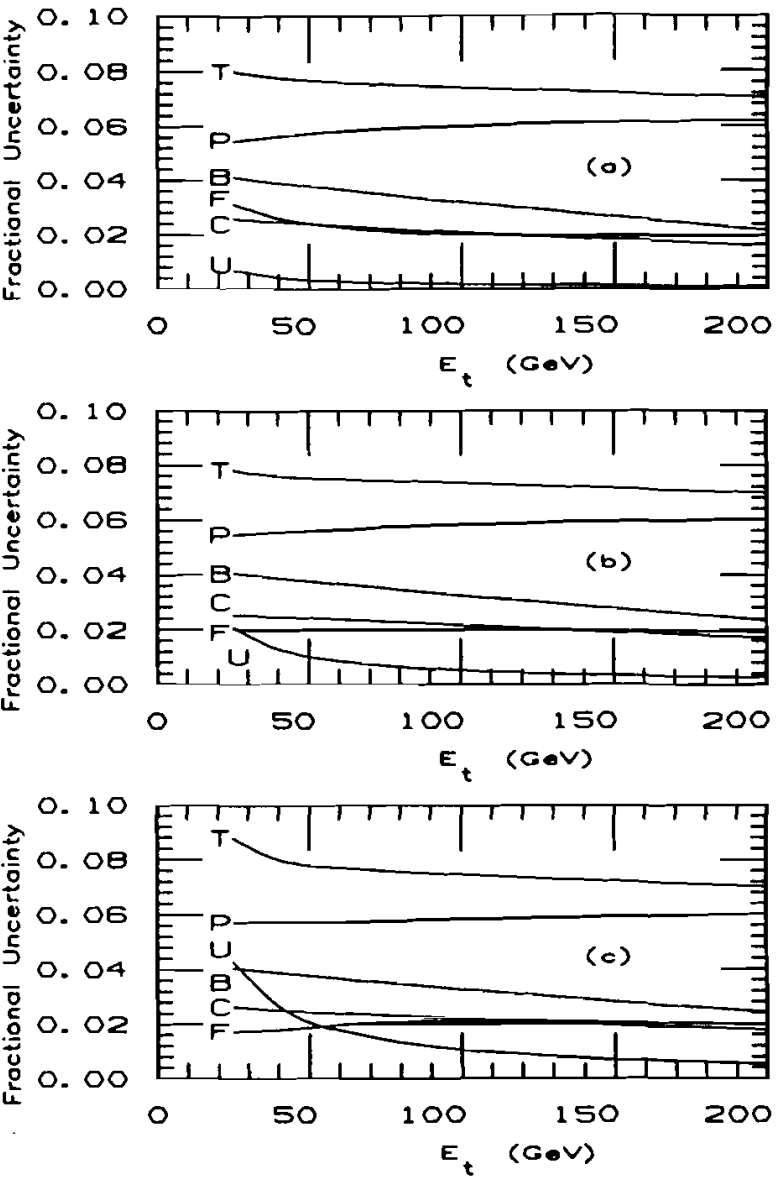

Figure 36. The breakdown of the various components of the total fractional systematic uncertainty on the corrected jet $E_{\mathbf{l}}$ scale for each cone size. For a) cone size of 0.4, b) cone size of 0.7 , and c) cone size of 1.0 where the letters represent the following uncertainties: total uncertainty $(T)$, azimuthal crack uncertainty (P), test beam uncertainty (B), calorimeter azimuthal crack uncertainty $(P)$, test beam uncertainty $(B)$, calorimeter
response $(C)$, fragmentation uncertainty $(F)$, and underlying event $(U)$. 
jet $E_{t}$ scale of $2 \%$ was added in quadrature to the resulting uncertainties listed in Table XXIII.

B. Systernatic Uncertainty on the Jet Resolution

As a check of the uncertainty on the jet resolution, dijet balancing techniques were used to measure the jet resolution. The uncertainties on the jet resolution due to the use of the simulation is related to the difference in the resolution ineasured using dijet balancing in both the data and simulation.

Dijet $k_{t}$ is the vector sum of the transverse momentuin in dijet events. It is shown in Appendix A that the width of the $k_{t_{\|}}\left(\sigma_{k_{t_{n}}}\right)$ and $k_{t_{\perp}}\left(\sigma_{k_{t_{\perp}}}\right)$ distributions are related to the jet resolution. $\sigma_{k_{k_{1}}}$ and $\sigma_{k_{t_{\perp}}}$ have been ineasured in dijet events for the data and simulation. This information was then used to calculate

$$
\sigma^{\prime}=\left(\sigma_{k_{1}}^{2}-\sigma_{k_{\perp}}^{2}\right)^{1 / 2}
$$

The width of the response functions measured in the simulation were parameterized by Sigma and Slope.(70) These numbers can be related to $\sigma^{\prime}$ by using the relation

$$
\sigma_{r m g}=\left(S i g m a^{2}+S l o p e^{2}\right)^{1 / 2}
$$

This relation expresses the width of the response functions in terms of a single number.

The single jet resolution is $\sigma^{\prime} / \sqrt{2}$ and is directly related to $\sigma_{r m}$ since both are a neasure of the width of the detectors response to jets. Taking the bin by bin difference in $\sigma^{\prime} / \sqrt{2}$ for the data and sinulation gives the systematic difference in the jet resolution. This difference was then fitted to a straight line (parameterized by slope $(m)$ and of set $\left._{(b)}\right)$ in order to paranieterize this difference. The errors on the $m$ and $b$ for this line represent the statistical level of uncertainty on this difference.

('hanging the parameterized fit by the errors on the $m$ and $b$, and adding or sulbtracting the result with the absolute value of the original fit, a band of uncertainty associated with the jet resolution was obtained. Figure 37 slows this result for cone sizes of $0.4,0.7$ and 1.0 .

C. Luminosity Uncertainty

The luminosity equals the interaction rate over the eflective cross section producing the Beam-13eam counter rates $\left(\sigma_{B B C}^{e f f}\right)$. At $\sqrt{s}=1.8 \mathrm{TeV}$, previous calculations of $\sigma_{B B C}^{e f f}$ depended on the extrapolation of measurements made at the SPS at CERN. $[80,81,82]$

The luminosity calculated using the Beam-Beam counters is expressed as follows,

$$
L_{B B C}=\frac{N_{B B C}}{\sigma_{B B C}^{e f f}}
$$

The luminosity can also be calculated using accelerator parameters by means of the following expression,

$$
L_{A c c}=B \frac{N_{p} N_{\bar{p}}}{4 \pi \sigma_{x} \sigma_{y}} f
$$

where $B$ is the number of bunches, $N_{p}$ and $N_{\bar{p}}$ is the number of protons and antiprotons respectively, $\sigma_{x}$ and $\sigma_{y}$ are the horizontal and vertical size of the bunch at the interaction point, and $f$ is the revolution frequency of the beams. 

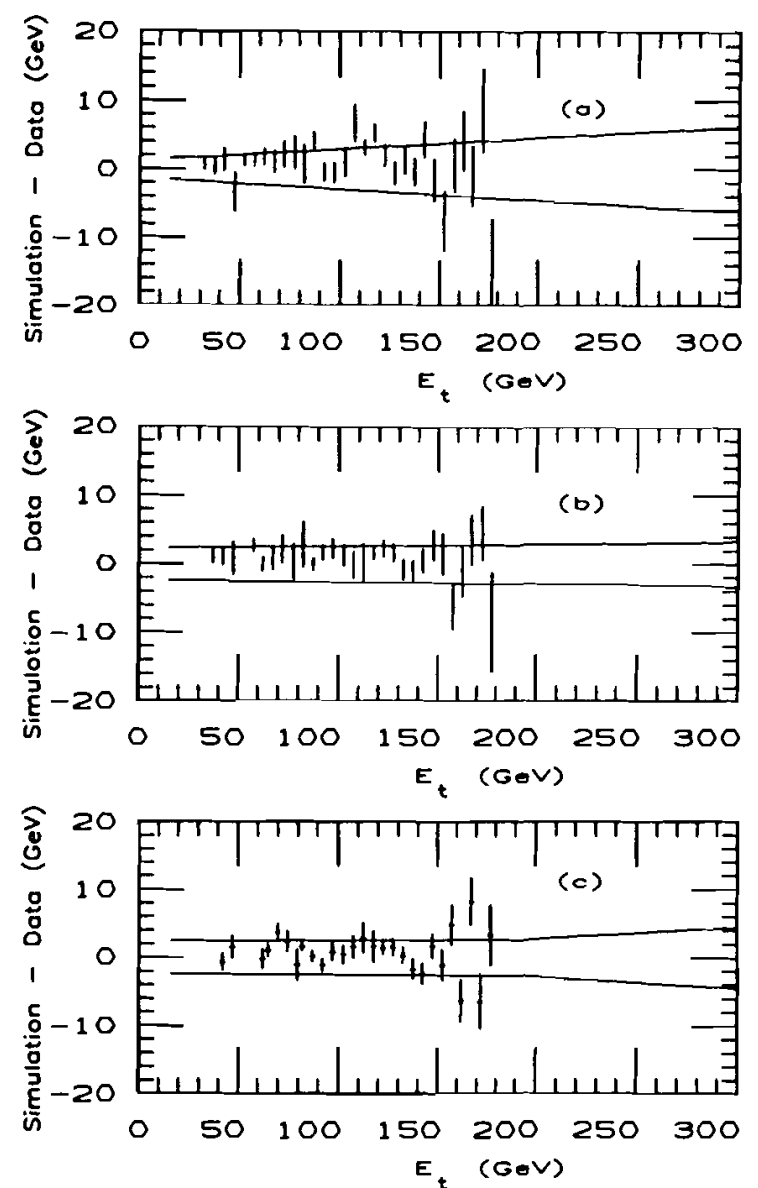

Figure 37. Difference in the rms jet resolution between data and simulation. The lines represent the band of uncertainty associated with this measureinent. a) cone size of $0.4, b)$ cone size of 0.7 , and c) cone size of 1.0
'The ratio of $L_{B B C}(5.16) / L_{A c c}(546)$ and $L_{B B C}(1800) / L_{A C c}(1800)$ were measured to be $0.753 \pm 0.002$ and $0.981 \pm 0.001$ respectively.[83] The effective Beam-Bean counter cross section at $\sqrt{s}=1.8 \mathrm{TeV}$ can be expressed by the following equation,

$$
\sigma_{B B C}^{e f f}(1800)=\sigma_{B B C}^{e f f}(546) \frac{L_{B B C}(1800) / L_{A C C}(1800)}{L_{B B C}(546) / L_{A C C}(546)}
$$

The value of $\sigma_{B B C}^{e f f}(546)$ has been measured both by the accelcrator and UA4 detector to be $(32.8 \pm 3.6) \mathrm{mb}$ and $(37.1 \pm(5.6 \%)) \mathrm{mb}$ respectively, for a combined average cross section of $(36.0 \pm 1.81) \mathrm{mb}$. This gives an effective Beam-Beann counter cross section at $\sqrt{\mathrm{s}}=1.8 \mathrm{TcV}$ of $(46.8 \pm 2.35 \pm 2.16) \mathrm{mb}$ with a total uncertainty of $6.8 \%$

b. Systematic Uncertainty on the Jet Cross Section

The systematic uncertaintics from the jet $E_{f}$ scale, resolution, and luminosity uncertainty have to be factored into the cross section. The jet $E_{1}$ scale and resolution are used in the unsmearing procedure to extract the corrected cross section from the data. 70$]$ Therefore, the jet $E_{l}$ scale and resolution can be varied in the unsmearing procedure to obtain the size of these effects on the cross section. The uncertainty in the luminosity is a normalization uncertainty and was factored into the cross section directly.

The metliod used for extracting the uncertainty on the cross section arising from the uncertainty on the jet $E_{t}$ scale and resolution is as follows. The parameterized curve obtained for the corrected cross section is input into the smearing procedure[70] and then the jet $E_{\ell}$ scale and resolution are varied separately within their systematic 
uncertainties. New corrections are extracted without minimizing the parameterized curve. These new corrections show the size and direction of the systematic uncertainty under study on the cross section. The fractional uncertainty on the cross section is then defined to be the ratio of the difference in the cross section calculated with these new corrections and parameterized curve used as input. This result is then fit to a suitahle function, in order to parameterize the fractional uncertanty on the cross section as a function of jet $E_{t}$. The results obtained for cach cone size when the jet $E_{\mathrm{t}}$ scale was varied are plotted in Figure 38 . These results are plotted in terıns of the ratio of the uncertainty and the predicted cross section obtained from the parameterized curve. The results obtained for each cone size when the jet resolution was varicd are plotted in Figure 39. These results are also plotted in terıns of the ratio of the uncertainty and the predicted cross section from the parameterized curve. manner as the results for the jet $E_{\mathrm{t}}$ uncertainty.

The uncertainty on the cross section due to the $E_{l}$ scale and jet resolution are then added in quadrature with the uncertainty on the Juninosity to obtain the full systematic uncertainty on the inclusive jet cross section. 'The band of systematic uncertainty on the cross section can be seen in Figure 40 for all thrce cone sizes where it has been plotted as a ratio of the total uncertainty and the predicted cross section from the parameterized curvc. Table XXIV, XXV, and XXVI list the systematic uncertaintics for each point in the cross section for conc sizes of $0.4,0.7$, and 1.0 respectively.
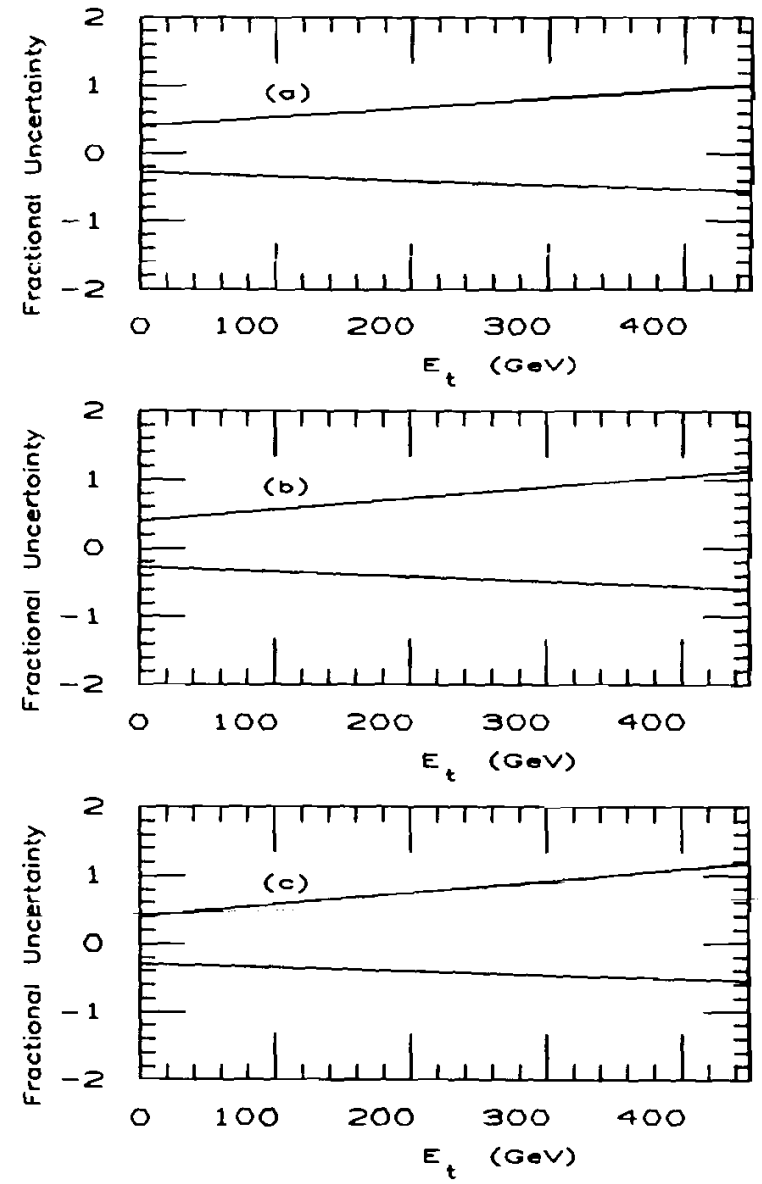

l-igure 38. The fraction of uncertainty on the jet cross section, from the uncertainty on the $E_{t}$ scalc. Results are shown for a) cone size of 0.4, b) cone size of 0.7 and c) cone size of 1.0 . 

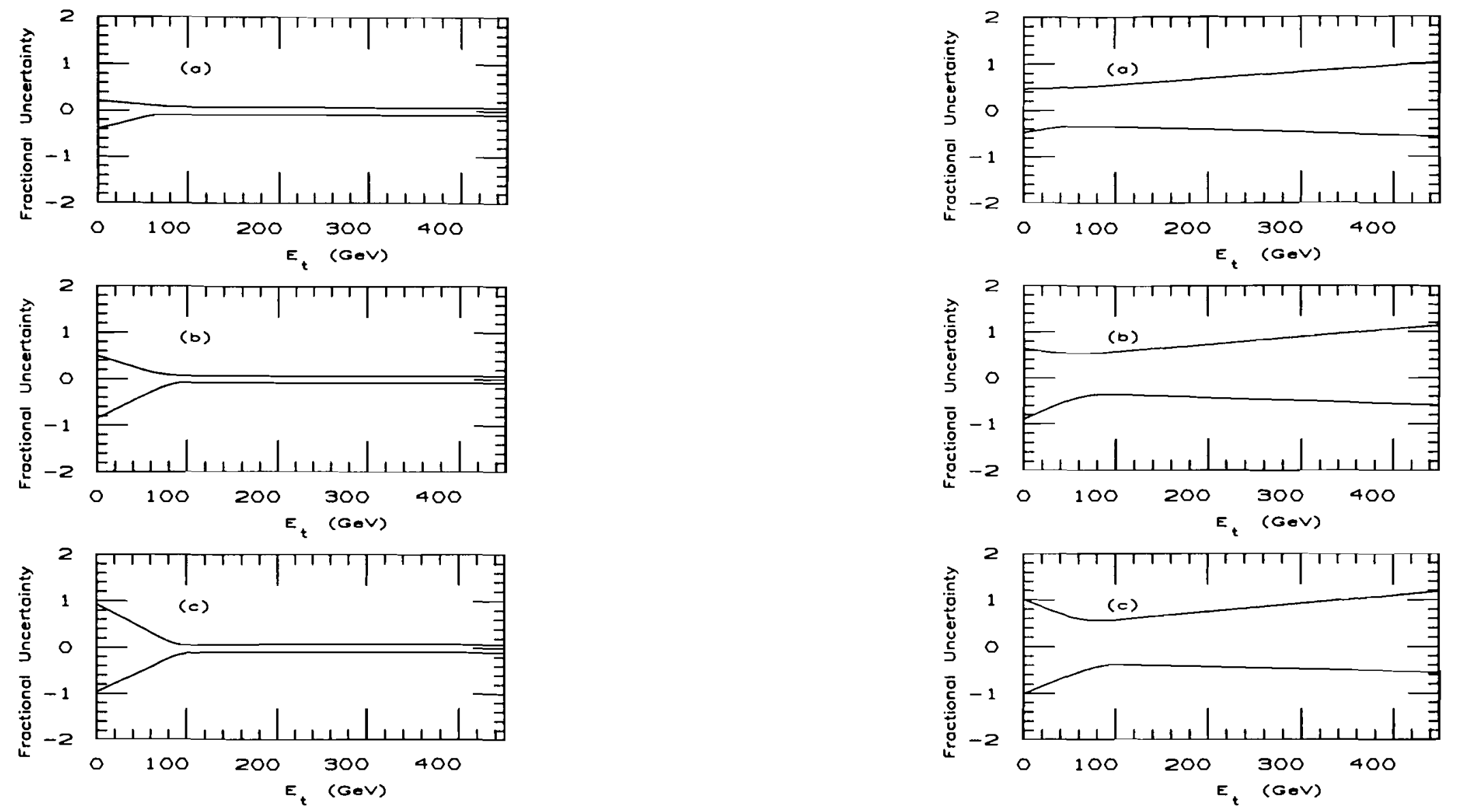

Pigure 39. The fraction of uncertainty on the jet cross section, from the uncertainty on the jet resolution. Results are shown for a) cone size of $0.4, \mathrm{~b}$ ) cone size of 0.7 and c) cone sizc of 1.0

Figure 40. The total fraction of systematic uncertainty on the jet cross section. Results are slıown for a) cone size of $0.4, \mathrm{~b}$ ) cone size of 0.7 and c) cone size of 1.0 . 
Table XXIV. Percent systematic uncertainty on the inclusive jet cross section for a jet clustering cone size of 0.4

\begin{tabular}{|c|c|c|}
\hline \multirow{2}{*}{$\begin{array}{c}\text { Mean } \\
E_{t}(\mathrm{GeV})\end{array}$} & \multicolumn{2}{|c|}{ Systematic Uncertainties } \\
\hline & $E_{1}$ Dependent (\%) & Total (\%) \\
\hline 36.24 & $\begin{array}{l}+15.30 \\
-4.13\end{array}$ & $\begin{array}{l}+48.58 \\
-37.21\end{array}$ \\
\hline 41.90 & $\begin{array}{l}+15.93 \\
-2.93\end{array}$ & $\begin{array}{l}+49.01 \\
-36.00\end{array}$ \\
\hline 47.52 & $\begin{array}{l}+16.39 \\
-1.89\end{array}$ & $\begin{array}{l}+49.46 \\
{ }_{-34.97}\end{array}$ \\
\hline 53.27 & $\begin{array}{r}+16.88 \\
-1.02\end{array}$ & $\begin{array}{r}+19.96 \\
-34.10\end{array}$ \\
\hline 59.01 & $\begin{array}{r}+17.39 \\
-0.35\end{array}$ & $\begin{array}{l}+50.47 \\
-33.43\end{array}$ \\
\hline 64.53 & $\begin{array}{l}+17.92 \\
-0.00\end{array}$ & $\begin{array}{l}+50.99 \\
-33.08\end{array}$ \\
\hline 69.85 & $\begin{array}{l}+18.44 \\
-0.32\end{array}$ & $\begin{array}{l}+51.52 \\
-33.40\end{array}$ \\
\hline 75.30 & $\begin{array}{l}+19.00 \\
-0.65\end{array}$ & $\begin{array}{l}+52.08 \\
-33.73\end{array}$ \\
\hline 80.62 & $\begin{array}{r}+19.57 \\
-098\end{array}$ & $\begin{array}{l}+52.65 \\
-3405\end{array}$ \\
\hline 85.78 & $\begin{array}{r}+20.11 \\
-1.29\end{array}$ & $\begin{array}{r}+53.22 \\
-34.37\end{array}$ \\
\hline 91.12 & $\begin{array}{r}+20.83 \\
-1.61\end{array}$ & $\begin{array}{l}+33.91 \\
-34.69\end{array}$ \\
\hline 96.33 & ${ }_{-1.93}^{+21.53}$ & $\begin{array}{l}+54.61 \\
-35.01\end{array}$ \\
\hline 101.64 & $\begin{array}{l}+22.25 \\
-2.26\end{array}$ & $\begin{array}{l}+55.33 \\
-35.33\end{array}$ \\
\hline 106.88 & $\begin{array}{l}+22.96 \\
-2.57\end{array}$ & $\begin{array}{l}+36.04 \\
-35.65\end{array}$ \\
\hline 112.08 & $\begin{array}{r}+23.66 \\
-2.89\end{array}$ & $\begin{array}{l}+56.74 \\
-35.97\end{array}$ \\
\hline 117.30 & $\begin{array}{r}+21.37 \\
-3.21\end{array}$ & $\begin{array}{r}+5745 \\
-36.29\end{array}$ \\
\hline 122.74 & $\begin{array}{l}+23.11 \\
+3.54\end{array}$ & $\begin{array}{r}+58.19 \\
-36.62\end{array}$ \\
\hline 127.94 & ${ }_{-3.86}^{+25.81}$ & $\begin{array}{l}+58.89 \\
{ }_{-36.94}\end{array}$ \\
\hline 133.12 & $\begin{array}{r}+26.51 \\
-1.18\end{array}$ & $\begin{array}{l}+59.59 \\
-37.26\end{array}$ \\
\hline 138.30 & $\begin{array}{r}+27.22 \\
-6.50\end{array}$ & $\begin{array}{l}+60.29 \\
-37.58\end{array}$ \\
\hline 143.46 & $\begin{array}{r}+27.91 \\
-1.81\end{array}$ & $\begin{array}{l}+60.99 \\
-37.89\end{array}$ \\
\hline 148.83 & $\begin{array}{l}+28.64 \\
-5.14\end{array}$ & $\begin{array}{l}+61.72 \\
-38.22\end{array}$ \\
\hline 153.88 & $\begin{array}{l}+29.33 \\
-5.46\end{array}$ & $\begin{array}{l}+62.41 \\
-38.53\end{array}$ \\
\hline
\end{tabular}

Table XXIV. Continued

\begin{tabular}{|c|c|c|}
\hline Mean & Systematic Unc & ertainties \\
\hline$E_{t}(\mathrm{GeV})$ & Et Dependent (\%) & Total (\%) \\
\hline 159.07 & $\begin{array}{l}+30.03 \\
-3.77\end{array}$ & $\begin{array}{l}+63.11 \\
-38.85\end{array}$ \\
\hline 166.90 & $\begin{array}{c}+31.10 \\
-6.26\end{array}$ & $\begin{array}{l}+64.17 \\
{ }_{-39.34}\end{array}$ \\
\hline iร̃̄ồ & $\begin{array}{l}+32.47 \\
-6.88\end{array}$ & $\begin{array}{r}\mathbf{+ 6 5 . 5 5} \\
-\mathbf{3 9 . 9 6}\end{array}$ \\
\hline 187.49 & $\begin{array}{l}+33.89 \\
-7.53\end{array}$ & $\begin{array}{l}+66.97 \\
-40.61\end{array}$ \\
\hline 198.23 & $\begin{array}{l}+35.35 \\
-8.19\end{array}$ & $\begin{array}{l}+68.43 \\
-41.27\end{array}$ \\
\hline 210.38 & $\begin{array}{l}+37.01 \\
-8.95\end{array}$ & $\begin{array}{l}+70.08 \\
-12.02\end{array}$ \\
\hline 227.23 & $\begin{array}{l}+39.30 \\
-9.99\end{array}$ & $\begin{array}{l}+72.38 \\
-43.02\end{array}$ \\
\hline 241.41 & $\begin{array}{l}+11.23 \\
-10.87\end{array}$ & $\begin{array}{l}+71.31 \\
-43.95\end{array}$ \\
\hline 259.30 & $\begin{array}{l}+43.66 \\
-11.98\end{array}$ & $\begin{array}{l}+76.71 \\
+-45.06\end{array}$ \\
\hline 277.46 & $\begin{array}{l}+46.14 \\
-13.12\end{array}$ & $\begin{array}{l}+79.22 \\
-46.19\end{array}$ \\
\hline 299.20 & $\begin{array}{l}+49.10 \\
-14.47\end{array}$ & $\begin{array}{l}+82.18 \\
-17.55\end{array}$ \\
\hline 317.64 & $\begin{array}{l}\mathbf{+} 51.62 \\
-15.63\end{array}$ & $\begin{array}{l}+84.70 \\
-18.70\end{array}$ \\
\hline 338.92 & $\begin{array}{c}+54.52 \\
-16.96\end{array}$ & $\begin{array}{l}+87.60 \\
-50.04\end{array}$ \\
\hline 371.08 & $\begin{array}{l}+58.91 \\
-18.98\end{array}$ & $\begin{array}{r}+91.99 \\
-52.06\end{array}$ \\
\hline 409.19 & $\begin{array}{l}+64.12 \\
+21.38 \\
-2.3\end{array}$ & $\begin{array}{r}+97.20 \\
-54.45 \\
\end{array}$ \\
\hline
\end{tabular}


Table XXV. Percent systematic uncertainty on the inclusive jet cross section for a jet clustering cone size of 0.7 .

\begin{tabular}{|c|c|c|}
\hline \multirow{2}{*}{$\begin{array}{c}\text { Mean } \\
E_{t}(\mathrm{GeV})\end{array}$} & \multirow{2}{*}{$\begin{array}{c}\text { Systematic Un } \\
E_{l} \text { Dependent }(\%)\end{array}$} & \multirow{2}{*}{$\begin{array}{l}\text { Total (\%) } \\
\text { Tointies }\end{array}$} \\
\hline & & \\
\hline 41.63 & $\begin{array}{l}+18.43 \\
-20.16\end{array}$ & $\begin{array}{r}+53.45 \\
{ }_{-55.18}\end{array}$ \\
\hline 47.61 & $\begin{array}{r}+17.73 \\
-15.82\end{array}$ & $\begin{array}{l}+52.75 \\
-50.84\end{array}$ \\
\hline 53.54 & $\begin{array}{l}+17.37 \\
-11.80\end{array}$ & $\begin{array}{r}+52.29 \\
-46.82\end{array}$ \\
\hline 59.93 & $\begin{array}{r}+17.04 \\
-7.89\end{array}$ & $\begin{array}{l}+52.06 \\
-42.91\end{array}$ \\
\hline 66.23 & $\begin{array}{l}+17.10 \\
+-1.57\end{array}$ & $\begin{array}{l}+32.12 \\
+39.59\end{array}$ \\
\hline 72.29 & $\begin{array}{r}+17.41 \\
-2.03\end{array}$ & $\begin{array}{l}+52.43 \\
{ }_{-37.05}\end{array}$ \\
\hline 78.15 & $\begin{array}{r}+18.19 \\
-0.30\end{array}$ & $\begin{array}{l}+53.21 \\
-35.32\end{array}$ \\
\hline 83.81 & $\begin{array}{r}+19.11 \\
+0.00\end{array}$ & $\begin{array}{r}+55.13 \\
+535.02\end{array}$ \\
\hline 89.30 & $\begin{array}{r}+20.01 \\
-0.36\end{array}$ & $\begin{array}{r}+55.02 \\
-35.38\end{array}$ \\
\hline 94.82 & $\begin{array}{r}+20.90 \\
-0.72\end{array}$ & $\begin{array}{r}+55.92 \\
-35.74\end{array}$ \\
\hline 100.19 & $\begin{array}{r}+21.78 \\
-1.08\end{array}$ & $\begin{array}{r}+36.80 \\
-36.09\end{array}$ \\
\hline 105.59 & $\begin{array}{r}+22.60 \\
+-1.43\end{array}$ & $\begin{array}{r}+57.67 \\
-36.15\end{array}$ \\
\hline 111.04 & $\begin{array}{l}+23.54 \\
+-1.79\end{array}$ & $\begin{array}{r}+58.56 \\
-36.81\end{array}$ \\
\hline 116.44 & $\begin{array}{l}+24.12 \\
-2.14\end{array}$ & $\begin{array}{l}+59.41 \\
{ }_{-37.16}\end{array}$ \\
\hline 121.76 & $\begin{array}{r}+25.29 \\
-2.50\end{array}$ & $\begin{array}{r}+60.31 \\
+-37.51\end{array}$ \\
\hline 127.12 & $\begin{array}{l}+26.16 \\
-2.85\end{array}$ & $\begin{array}{r}+61.18 \\
-37.87\end{array}$ \\
\hline 132.49 & $\begin{array}{r}+27.04 \\
-3.20\end{array}$ & $\begin{array}{r}+62.06 \\
-38.22\end{array}$ \\
\hline 137.77 & $\begin{array}{r}+27.90 \\
+27.55\end{array}$ & $\begin{array}{r}+62.92 \\
{ }_{-38}^{6.57}\end{array}$ \\
\hline 143.05 & $\begin{array}{r}+28.76 \\
+-3.90\end{array}$ & $\begin{array}{r}+63.78 \\
{ }_{-38.92}\end{array}$ \\
\hline 148.48 & $\begin{array}{r}+29.65 \\
+4.26\end{array}$ & $\begin{array}{l}+61.67 \\
{ }_{-39.28}\end{array}$ \\
\hline 153.71 & $\begin{array}{r}+30.50 \\
+-1.61\end{array}$ & $\begin{array}{l}+65.52 \\
-39.63\end{array}$ \\
\hline 158.92 & $\begin{array}{r}+31.36 \\
-1.95 \\
-1.95\end{array}$ & $\begin{array}{l}+66.37 \\
{ }_{-39.97}\end{array}$ \\
\hline 164.24 & $\begin{array}{r}+32.33 \\
+5.31 \\
-5.31\end{array}$ & $\begin{array}{l}+67.24 \\
+-10.33\end{array}$ \\
\hline
\end{tabular}

Table XXV. Continued

\begin{tabular}{|c|c|c|}
\hline \multirow{2}{*}{$\begin{array}{c}\text { Mean } \\
E_{\ell}(\mathrm{GeV})\end{array}$} & Systematic & Uncertainties \\
\hline & $E_{t}$ Dependent (\%) & Tolal (\%) \\
\hline 171.89 & $\begin{array}{l}+33.48 \\
-5.81\end{array}$ & $\begin{array}{r}+68.49 \\
-40.83\end{array}$ \\
\hline 182.20 & $\begin{array}{l}+35.16 \\
{ }_{-6.50}\end{array}$ & $\begin{array}{l}+70.18 \\
-41.52\end{array}$ \\
\hline 193.03 & $\begin{array}{r}+36.93 \\
{ }_{-7.22}\end{array}$ & $\begin{array}{l}+71.95 \\
-42.24\end{array}$ \\
\hline 203.47 & $\begin{array}{l}+38.64 \\
-7.92\end{array}$ & $\begin{array}{l}+73.66 \\
-12.93\end{array}$ \\
\hline 215.73 & $\begin{array}{r}+40.65 \\
-8.73\end{array}$ & $\begin{array}{l}+75.67 \\
-43.75\end{array}$ \\
\hline 231.87 & $\begin{array}{r}+43.30 \\
+-9.81\end{array}$ & $\begin{array}{r}+78.32 \\
+-11.83\end{array}$ \\
\hline 246.86 & $\begin{array}{r}+45.75 \\
-10.81\end{array}$ & $\begin{array}{l}+80.77 \\
-45.83\end{array}$ \\
\hline 264.86 & $\begin{array}{r}+18.71 \\
-12.02\end{array}$ & $\begin{array}{l}+83.72 \\
-47.04\end{array}$ \\
\hline 281.96 & $\begin{array}{l}+51.51 \\
-13.17\end{array}$ & $\begin{array}{l}+86.53 \\
{ }_{-48.19}\end{array}$ \\
\hline 302.22 & $\begin{array}{r}+54.84 \\
-14.53\end{array}$ & $\begin{array}{l}+89.86 \\
-49.55\end{array}$ \\
\hline 322.87 & $\begin{array}{r}+58.23 \\
-15.92\end{array}$ & $\begin{array}{r}+93.25 \\
-50.94\end{array}$ \\
\hline 343.87 & $\begin{array}{r}+61.68 \\
-17.33\end{array}$ & $\begin{array}{r}+96.70 \\
-52.35\end{array}$ \\
\hline 380.71 & $\begin{array}{r}+67.74 \\
-19.82\end{array}$ & $\begin{array}{r}+102.76 \\
-54.84\end{array}$ \\
\hline 418.54 & $\begin{array}{l}+73.96 \\
{ }_{-22.37}\end{array}$ & $\begin{array}{r}+108.98 \\
-57.39 \\
\end{array}$ \\
\hline
\end{tabular}


Table XXVI. Percent systematic uncertainty on the inclusive jet cross section for a jet clustering cone size of 1.0 .

\begin{tabular}{|c|c|c|}
\hline Mean & Systematic Unce & ertainties \\
\hline$E_{l}(\mathrm{GeV})$ & $E_{t}$ Dependent (\%) & Total (\%) \\
\hline 45.78 & $\begin{array}{l}+30.81 \\
-27.55\end{array}$ & $\begin{array}{l}+67.99 \\
-64.73\end{array}$ \\
\hline 53.75 & $\begin{array}{l}+26.55 \\
{ }_{-21.78}\end{array}$ & $\begin{array}{l}+63.73 \\
{ }_{-58.96}\end{array}$ \\
\hline 61.29 & $\begin{array}{r}+23.23 \\
-16.62\end{array}$ & $\begin{array}{r}+60.41 \\
-53.81\end{array}$ \\
\hline 68.10 & $\begin{array}{l}+20.93 \\
-12.31\end{array}$ & $\begin{array}{r}+58.11 \\
-49.49\end{array}$ \\
\hline 74.55 & $\begin{array}{l}+19.44 \\
-8.59\end{array}$ & $\begin{array}{l}+56.62 \\
-45.77\end{array}$ \\
\hline 80.41 & $\begin{array}{l}+18.71 \\
-5.61\end{array}$ & $\begin{array}{l}+55.89 \\
-42.79\end{array}$ \\
\hline 86.48 & $\begin{array}{l}+18.65 \\
-3.03\end{array}$ & $\begin{array}{l}+55.83 \\
{ }_{-40.21}\end{array}$ \\
\hline 92.77 & $\begin{array}{l}+19.72 \\
-0.99\end{array}$ & $\begin{array}{l}+56.90 \\
-38.17\end{array}$ \\
\hline 98.70 & $\begin{array}{l}+20.73 \\
-0.00\end{array}$ & $\begin{array}{l}+57.91 \\
-37.18\end{array}$ \\
\hline 104.29 & $\begin{array}{r}+21.69 \\
-0.29\end{array}$ & $\begin{array}{l}+58.87 \\
-37.47\end{array}$ \\
\hline 109.90 & $\begin{array}{r}+22.65 \\
-0.58\end{array}$ & $\begin{array}{r}+59.83 \\
-37.76\end{array}$ \\
\hline 115.45 & $\begin{array}{l}+23.59 \\
-0.86\end{array}$ & $\begin{array}{l}+60.77 \\
-38.05\end{array}$ \\
\hline 120.89 & $\begin{array}{r}+24.53 \\
-1.15\end{array}$ & $\begin{array}{l}+66.71 \\
-38.33\end{array}$ \\
\hline 126.24 & $\begin{array}{r}+25.44 \\
-1.42\end{array}$ & $\begin{array}{r}+62.62 \\
-38.60\end{array}$ \\
\hline 131.64 & $\begin{array}{r}+26.36 \\
-1.70\end{array}$ & $\begin{array}{r}+63.54 \\
-38.88\end{array}$ \\
\hline 137.04 & $\begin{array}{r}+27.29 \\
-1.98\end{array}$ & $\begin{array}{l}+61.47 \\
-39.16\end{array}$ \\
\hline 142.37 & $\begin{array}{r}+28.20 \\
+2.26\end{array}$ & $\begin{array}{l}+65.38 \\
-39.14\end{array}$ \\
\hline 147.79 & $\begin{array}{r}+29.13 \\
-2.54\end{array}$ & $\begin{array}{l}+66.31 \\
{ }_{-39.72}\end{array}$ \\
\hline 153.12 & $\begin{array}{r}+30.04 \\
-2.82\end{array}$ & $\begin{array}{l}+67.23 \\
-40.00\end{array}$ \\
\hline 158.53 & $\begin{array}{l}+30.97 \\
-3.10\end{array}$ & $\begin{array}{l}+68.15 \\
-40.28\end{array}$ \\
\hline 163.91 & $\begin{array}{r}+31.90 \\
+3.38\end{array}$ & $\begin{array}{l}+69.08 \\
-40.56\end{array}$ \\
\hline 171.74 & $\begin{array}{l}+33.24 \\
+-3.78\end{array}$ & $\begin{array}{l}+70.42 \\
-40.97\end{array}$ \\
\hline 182.16 & $\begin{array}{r}+35.03 \\
-4.33\end{array}$ & $\begin{array}{l}+72.21 \\
-41.51\end{array}$ \\
\hline
\end{tabular}

Table XXVI. Continued

\begin{tabular}{|c|c|c|}
\hline Mean & Systematic Unc & ertainties \\
\hline$E_{6}(\mathrm{GeV})$ & $E_{t}$ Dcpendent (\%) & Total $(\%)$ \\
\hline 193.07 & $\begin{array}{r}+36.90 \\
-4.90\end{array}$ & $\begin{array}{r}+74.08 \\
-42.08\end{array}$ \\
\hline 203.44 & $\begin{array}{l}+38.68 \\
-5.44\end{array}$ & $\begin{array}{l}+75.86 \\
-42.62\end{array}$ \\
\hline 216.42 & $\begin{array}{l}+40.91 \\
-6.12\end{array}$ & $\begin{array}{l}+78.10 \\
-43.30\end{array}$ \\
\hline 233.06 & $\begin{array}{l}+43.78 \\
-6.99\end{array}$ & $\begin{array}{l}+80.96 \\
-11.17\end{array}$ \\
\hline 217.46 & $\begin{array}{l}\mathbf{+}+6.25 \\
-7.74\end{array}$ & $\begin{array}{l}+83.43 \\
-11.92\end{array}$ \\
\hline 264.64 & $\begin{array}{l}+19.21 \\
-8.64\end{array}$ & $\begin{array}{r}+86.39 \\
{ }_{-45.82}\end{array}$ \\
\hline 282.03 & $\begin{array}{l}+52.21 \\
-9.55\end{array}$ & $\begin{array}{l}+89.39 \\
-46.74\end{array}$ \\
\hline 305.43 & $\begin{array}{l}+56.24 \\
-10.79\end{array}$ & $\begin{array}{l}+93.42 \\
-47.97\end{array}$ \\
\hline 326.53 & $\begin{array}{l}+59.88 \\
-11.90\end{array}$ & $\begin{array}{r}+97.06 \\
-49.08\end{array}$ \\
\hline 348.63 & $\begin{array}{l}+63.69 \\
-13.06\end{array}$ & $\begin{array}{r}+100.88 \\
-50.24\end{array}$ \\
\hline 377.4 .4 & $\begin{array}{l}+68.67 \\
-14.38\end{array}$ & $\begin{array}{l}+105.85 \\
-51.76\end{array}$ \\
\hline 427.84 & $\begin{array}{r}+77.37 \\
-17.23 \\
\end{array}$ & $\begin{array}{r}+114.55 \\
+54.43 \\
\end{array}$ \\
\hline
\end{tabular}




\section{CHAPTER VI}

\section{COMPARISONS WITH QCD AND A COMPOSITE MODEL}

The measured inclusive jet cross section can be compared to predictions made by QCD and composite inodels.[19] Composite calculations for the inclusive jet cross section agree with QCD calculations at low values of $E_{l}$, but as the $E_{l}$ increases they start to deviate from one another. The comparison between the data and these models of parton-parton interactions may be used to scarch for evidence of quark substructure. Below, the procedures followed to compare the data with QCD and to extend the limits on the composite parameter $\Lambda_{c}$ are described in detail.

\section{A. Fitting Procedure}

In order to compare the measured cross section to theoretical predictions, a fitting procedure was developed to normalize a theoretical cross section to the measured cross section. This fitting procedure had to be able to normalize a theoretical cross section (either QCD or composite models) to the measured cross section in the presence of correlated systematic uncertaintics, and in some cases, the prescnce of large statistical errors in the data. The procedure described in this section attempts to account for both of these effects.

Theoretical points for the inclusive jet cross section are first calculated at the mean $E_{\ell}$ for each measured data point. To account for the fact that no jets are observed in the data above the highest $E_{l}$ bin, the integrated cross section above the point at which there is no dala is also calculated. This is referred to as the empty or Inull bin in the data.

Normalization of the theoretical distribution to the data is then accomplished using both the statistical crrors and systenatic uncertainties on the cross section for the data, no attempt is made to account for any theoretical uncertainty. The systernatic uncertainty is separated into two parts, an $E_{t}$ dependent part and an $E_{t}$ independent part. The $E_{t}$ independent part is treated as a normalization uncertainty on the data and the $E_{t}$ dependent part is assumed to be totally correlated bin to bin. This assumption is based on the belief that a systematic uncertainty in one bin of the data is strongly correlated to the systematic uncertainties in the other bins since the source of these uncertainties are the same. This implies that if the cross section in onc bin of the data is moved up or down, the cross section in the other bins move up or down coherently.

In order to find the normalization in some region of $E_{t}$, the sum of the bin by bin $x^{2}$ is calculated in that region and the normalization is adjusted in order to minimize this $\chi^{2}$

In the bins containing more than twenty jets in both the data and the theoretical calculation, the $\chi^{2}$ is calculated using the statistical errors combined with the systematic uncertainties on the data. The number twenty was chosen for this cutof in order to be consistent with the use of Poisson statistics in the calculation of the statistical errors on the cross section (sce Chapter III). In matrix notation the $\chi^{2}$ for bins with greater than twenty jets is represented as follows,

$$
\chi^{2}=\vec{\Delta}^{\prime} V^{-1} \vec{\Delta}
$$


where

$$
\vec{\Delta}=\left(\begin{array}{c}
\Delta_{1} \\
\Delta_{2} \\
\vdots \\
\Delta_{1}
\end{array}\right)
$$

and

$$
V=\left(\begin{array}{cccc}
\sigma_{11}^{2} & \sigma_{12}^{2} & \ldots & \sigma_{11}^{2} \\
\sigma_{21}^{2} & \sigma_{22}^{2} & \ldots & \sigma_{2 l}^{2} \\
\vdots & \vdots & \ddots & \vdots \\
\sigma_{11}^{2} & \sigma_{12}^{2} & \ldots & \sigma_{11}^{2}
\end{array}\right)
$$

where $l$ is the number of bins containing more than twenty jets in the fit region. $\vec{\Delta}$ is a vector of differences between the theorctical prediction of the cross section and the measurcd cross section. Each clement in the vector represents one bin in $E_{\mathrm{l}}$. The matrix $V$ is the error matrix. The diagonal elernents are the sum of the squares for the statistical and $E_{t}$ dependent systematic uncertainties. These elements are defined as follows

$$
\sigma_{i i}^{2}=\sigma_{i}^{2}(s t a t .)+\sigma_{i}^{2}(s y s .)
$$

wliere $\sigma_{i}\left(\right.$ stat.) and $\sigma_{i}($ sys.) are the statistical errors and systenatic uncertainties on bin i respectively. The off-diagonal elements are a measure of the correlations between the bins represented by $i$ and $j$ and are defined to be

$$
\sigma_{i j}^{2}=\rho_{i,} \sigma_{i}(s y s) \sigma_{\lambda}(s y s) \text { for } i \neq j,
$$

where $\rho_{i j}$ is the correlation coefficient and $\sigma_{i}(s y s)$ and $\sigma_{j}($ sys $)$ represent the systemat ic uncertainty on bin $i$ and $j$ respectively. The correlation coefficients $\left(\rho_{i}\right)$ were defined to be one since the systematic uncertainties were assumed to be totally correlated from bin lo bin.
For the bins with twenty or less jets in either the data or the theoretical cal. culation (including the emply bin), the $\chi^{2}$ was formed based on a likelihood ratio test. [6:] In this likelihood ratio test the $\chi^{2}$ in each bin was defined separately to be

$$
\chi^{2}=-2 \ln (\lambda),
$$

wilere

$$
\lambda=\frac{e^{-q} q^{n}}{e^{-n_{n}}}
$$

The number of jets predicted by theory is represented by $q$, and $n$ is the number of jets observed in the data. Factoring Equation 44 after inserting Equation 45 results

$$
\chi^{2}=2\left((q-n)+n \ln \left(\frac{n}{q}\right)\right)
$$

This implies that the sum of the $x^{2}$ for all the bins with twenty or fewer jets can be defined to be

$$
\chi^{2}=\overline{2} \sum_{i=1}^{m}\left(\left(q_{i}-n_{i}\right)+n_{i} \ln \left(\frac{n_{i}}{q_{i}}\right)\right)
$$

where $m$ is the number of bins containing twenty or fewer jets.

The resulting $x^{2}$ in the fitted region for bins with greater than twenty jets and for bins with twenty jets or less are then combined to form the total $x^{2}$ in the fitled region. The $\chi^{2}$ in the regions not being used in the fit are also calculated in the same manner allowing these regions to be tested for variations in shape independently of the shape in the fitted region. 


\section{B. Comparisons to Leading Orde}

Both lcading order QCD and composite model calculations of the cross section are compared to the measured cross sertion in the same nanner. An appropriate region in $E_{\ell}$ is first chosen to normalize the theoretical prediction for the cross section to the data. A normalization region in $E_{1}$ is chosen for several reasons.

Leading order QCD and composite model predictions for the cross section start to deviate from one another above some valuc of $E_{l}$, but agree at lower values of $E_{t}$ the exact value of $E_{t}$ that this occurs is a function of the compositeness parameter $\Lambda_{c}$. For values of $\Lambda_{c} \geq 950 \mathrm{GeV}$ this deviation will occur above an $E_{t}$ of $160 \mathrm{GicV}$. Figure $4 \mathrm{I}$ shows this deviation in the predictions when using the structure function DFLM set I.

The systematic uncertainty on the cross section at low $E_{t}$ is large. In order to avoid having the normalization depend on the data in this region, a lower limit on the fit range was chosen to avoid this region. The fractional systematic uncertainty becomes smallest around $80 \mathrm{GeV}$ (see Chapter V), depending on the conc size under study, so a lower limit of $\sim 80 \mathrm{GeV}$ was chosen to avoid this region. Another reason for a lower limit on the fit range when comparing to leading order calculations is the effect of the correction for energy loss outside the clustering conc. When comparing to next-to-lcading order calculations, this correction is not appropriate and thercfore in this analysis was not applied. For leading order calculations this correction is appropriate. The size of this correction is largest for low values of $E_{t}$, thercfore when comparing to leading order calculations this can liave some effect. This is an

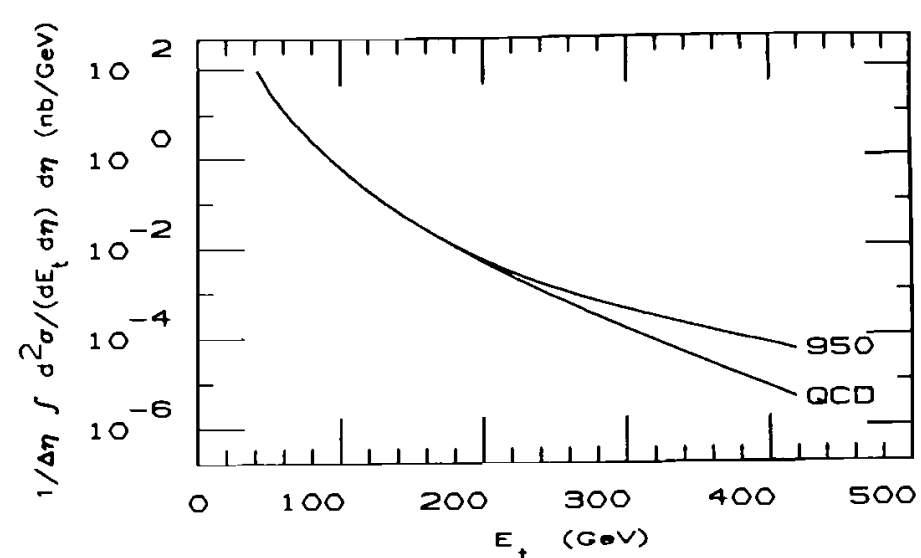

Figure 11. Leading order QCD calculation of the inclusive jet cross section comparcd to a Composite Model calculation using the structure function DFLM set 1 , with $\Lambda_{c}=950 \mathrm{GeV}$ and $Q^{2}=0.5 E_{t}^{2}$.

additional reason for having a lower limit on the range over which leading order calculations arc normalized to the data. Table XXVII is a list of the lower and upper $E_{t}$ limits used to define the fitting region for each cone size, as well as the lower and upper $E_{t}$ edge of the cross section observed in the data.

With the fit region defined, fits were done for leading order QCD and a Composite Model using a full range of structure functions. The structure functions are related to the probability of finding a parton with a particular momentum inside the proton (or antiproton) and have been measured in deep inelastic scattering experiments.[84] 
Table XXVII. List of the lower and upper limits defining the fitting region used for each cone size. Also listed are the lower and upper edges of the cross section observed inl the data.

\begin{tabular}{ccccc}
\hline & Fit Range $(\mathrm{GcV})$ & Data & Range $(\mathrm{GeV})$ \\
Cone Size & Lower Limit & Upper Limit & Lower Edge & Upper Edge \\
\hline 0.4 & 88.6 & 161.9 & 33.7 & 429.6 \\
0.7 & 81.2 & 161.7 & 38.9 & 440.0 \\
1.0 & 83.7 & 161.3 & 42.0 & 445.6 \\
\hline
\end{tabular}

A large number of different structure functions exist. The list of structure functions used in this analysis incluries Duke-Owens (DO, sets 1 and 2)[85] and EichtenHinchliffe-Lane-Quigg (E11LQ, sets 1 and 2)(27) structure functions which were obtained from the analysis of decp inclastic scattering data available prior to 1984 and used leading order QCD evolution. Diemoz-Ferroni-Longi-Martinelli (DILLM, sets I, 2 and 3)(86] Martin-Roberts-Stirling (MRS, sets 1, 2, 3, I3 and E)[87] structure functions are also used in this analysis and are based on newer experimental data available in 1988 using next-to-leading order QCD evolution. Tung-Morfin (TM, scts $1,2,3$ and 4$)[88]$ structure functions will also be examined in this analysis. Difterences within the various sets of structure functions mentioned above concern the treatment of the gluon distribution function and in some cases the use of different experimental data sets as input. In most cases, there exists one set which will use a broad or hard gluon distribution function and one set which uses a soft gluon distribution function.
Those which contain more than two sets will often vary the degree of hardness for this distribution between the two extremes for each set.

'The normalization was then found using the prescribed fitting procedure discussed earlier and $\chi^{2}$ 's are calculated in all relevant regions using this normalization. 1. Q(:1)

('omparisons to leading order QCD were made using the inclusive jet cross section measured with each of the three cone sizes $(0.4,0.7$, and 1.0$)$. Structure functions with a poor $\lambda^{2}$ in the fit region, which yielded a confidence level of less than $5 \%$, werc excluted from further use in this analysis (although the results obtained for these structure functions will be tabulated along with the other structure functions). The results of the fitting procedure for a cone size of 0.4 are shown in Table XXVIII. Table XXIX slows the saine results for a cone size of 0.7 and Table XXX contains the results for a cone size of 1.0 .

Using the remaining structure functions, the combined $x^{2}$ 's in the fit region, the lower region (bins below the fitted bins) and the upper region (bins above the fitted birrs) can be uscd to determine how well the data is deseribed by leading order QCD using the selected structure functions. Since the measured cross section had a large systematic uncertainty in the lower region and the correction for energy lost outside the clustering cone was not applied, the combined $\chi^{2}$ used to determine how well the data is described by leading order QCD will be based on only the $\chi^{2}$ in the fit region and the upper region.

Table XXX1 lists the combined $\chi^{2}$ (fit region combined with upper region), the number of bins (including the empty bin), and the confidence level obtained using 
Table XXVIII. The $x^{2}$ and number of bins found in the various regions examined in the fitting procedure for a cone size of 0.4 using the various structure functions.

\begin{tabular}{ccccccc}
\hline \hline \multirow{2}{*}{ Structure Fit Region } & \multicolumn{3}{c}{ Lower Region } & \multicolumn{3}{c}{ Upper Region } \\
Function & $\chi^{2}$ & NoB & $\chi^{2}$ & NoB & $\chi^{2}$ & Nol3 \\
\hline DO 1 & 11.75 & 14 & 7.00 & 10 & 16.10 & 15 \\
DO 2 & 12.68 & 14 & 232.52 & 10 & 22.90 & 15 \\
EIILQ 1 & 19.69 & 14 & 24.36 & 10 & 26.90 & 15 \\
EIILQ 2 & 24.61 & 14 & 10.08 & 10 & 42.13 & 15 \\
DFLM 1 & 27.40 & 14 & 34.23 & 10 & 41.19 & 15 \\
DFLM 2 & 27.15 & 14 & 75.40 & 10 & 42.82 & 15 \\
DFLM 3 & 24.85 & 14 & 115.01 & 10 & 39.69 & 15 \\
MRS 1 & 12.58 & 14 & 9.35 & 10 & 17.24 & 15 \\
MRS 2 & 9.16 & 14 & 146.09 & 10 & 14.30 & 15 \\
MRS 3 & 7.70 & 14 & 6.38 & 10 & 14.26 & 15 \\
MRS E & 13.08 & 14 & 11.30 & 10 & 17.72 & 15 \\
MRS B & 9.71 & 14 & 89.18 & 10 & 15.15 & 15 \\
TM 1 & 17.55 & 14 & 64.94 & 10 & 18.90 & 15 \\
TM 2 & 17.60 & 14 & 74.92 & 10 & 18.79 & 15 \\
TM 3 & 10.11 & 14 & 75.05 & 10 & 12.47 & 15 \\
TM 4 & 16.75 & 14 & 24.46 & 10 & 18.98 & 15 \\
\hline \hline & & & & & & \\
\hline
\end{tabular}

Table XXIX. The $x^{2}$ and number of bins found in the various regions examined in the fitting procedure for a cone size of 0.7 using the various structure functions.

\begin{tabular}{ccccccc}
\hline \hline \multirow{2}{*}{ Structure } & Fit Region & \multicolumn{2}{c}{ Lower Region } & \multicolumn{3}{c}{ Upper Region } \\
Function & $x^{2}$ & NoB & $\chi^{2}$ & NoB & $\chi^{2}$ & NoB \\
\hline DO 1 & 7.09 & 15 & 10.80 & 7 & 15.43 & 16 \\
DO 2 & 27.84 & 15 & 198.97 & 7 & 55.81 & 16 \\
EILQ 1 & 3.84 & 15 & 7.81 & 7 & 13.32 & 16 \\
EILQ 2 & 3.59 & 15 & 8.25 & 7 & 25.66 & 16 \\
DFLM 1 & 4.84 & 15 & 18.14 & 7 & 19.29 & 16 \\
DFLM 2 & 4.69 & 15 & 32.60 & 7 & 21.09 & 16 \\
DFLM 3 & 3.23 & 15 & 42.35 & 7 & 20.85 & 16 \\
MRS 1 & 6.60 & 15 & 8.95 & 7 & 15.43 & 16 \\
MRS 2 & 16.36 & 15 & 45.91 & 7 & 14.08 & 16 \\
MRS 3 & 12.03 & 15 & 7.20 & 7 & 16.40 & 16 \\
MRS E & 6.29 & 15 & 8.17 & 7 & 15.40 & 16 \\
MRS B & 18.23 & 15 & 35.64 & 7 & 15.37 & 16 \\
TM 1 & 3.22 & 15 & 12.13 & 7 & 11.55 & 16 \\
TM 2 & 3.10 & 15 & 13.90 & 7 & 11.52 & 16 \\
TM 3 & 5.56 & 15 & 12.52 & 7 & 19.63 & 16 \\
TM 4 & 3.84 & 15 & 8.03 & 7 & 11.43 & 16 \\
\hline
\end{tabular}


Table XXX. The: $x^{2}$ and number of bins found in the various regions examincel in the fitting procedure for a cone size of 1.0 using the various structure functions.

\begin{tabular}{ccccccc}
\hline \hline Structure & Fit Region & \multicolumn{3}{c}{ Lower } & \multicolumn{3}{c}{ Region } & \multicolumn{3}{c}{ Upper Hegion } \\
Function & $x^{2}$ & NoB & $x^{2}$ & NoB & $x^{2}$ & NoB \\
\hline DO 1 & 5.66 & 14 & 10.83 & 6 & 20.21 & 16 \\
DO 2 & 21.77 & 14 & 186.46 & 6 & 48.19 & 16 \\
EIILQ 1 & 4.79 & 14 & 11.55 & 6 & 25.00 & 16 \\
EIILQ 2 & 7.69 & 14 & 11.33 & 6 & 33.33 & 16 \\
DFLM 1 & 14.56 & 14 & 10.79 & 6 & 26.89 & 16 \\
DFLM 2 & 14.42 & 14 & 13.13 & 6 & 29.78 & 16 \\
DFLM 3 & 9.88 & 14 & 17.45 & 6 & 32.93 & 16 \\
MRS 1 & 5.50 & 14 & 10.49 & 6 & 21.39 & 16 \\
MRS 2 & 12.41 & 14 & 18.23 & 6 & 23.92 & 16 \\
MRS 3 & 11.96 & 14 & 10.99 & 6 & 23.91 & 16 \\
MRS E & 5.52 & 14 & 10.28 & 6 & 20.24 & 16 \\
MRS B & 13.09 & 14 & 22.06 & 6 & 23.20 & 16 \\
TM 1 & 5.86 & 14 & 11.68 & 6 & 21.72 & 16 \\
TM 2 & 5.95 & 14 & 11.94 & 6 & 21.78 & 16 \\
TM 3 & 6.51 & 14 & 10.56 & 6 & 23.31 & 16 \\
TM 4 & 5.42 & 14 & 11.27 & 6 & 18.36 & 16 \\
\hline \hline
\end{tabular}

the structure functions for a cone size of 0.4 . Table XXXII lists the same information for a cone size of 0.7 and Table XXX111 lists this information for a cone size of 1.0 . Thirse tables show the level of agreement between the measured cross section and the predicted leacling order QCD cross section. Figure 42 shows the data compared to leading order (QCI) using the structure function MRS set $B$ for a cone size of 0.4 ; t!ị infermation is plotted un both a iogaritimic and linear scale. The errors bars in this figure and all other figures, unless otherwise noted, are $E_{\mathfrak{t}}$ dependent systematic and statistical uncertainties added in quadrature. The normalization uncertainty and dashed liucs shown in these figures represent the $E_{t}$ independent systematic uncertainty on the cruss section. Figure $\mathbf{4 3}$ shows the same information for a cone size of 0.1 using the structure function MRS set 3 . Figure 44 shows the data compared to leading order QCD using the structure function MRS set B for a cone size of 0.7 and Figure 45 is a comparison of the data to leading order QCD for a cone size of 0.7 using the structure function MRS set 3 . Figure 46 shows the data compared to leading order $Q(D)$ using the structure function MRS set $B$ for a cone size of 1.0 and Figure 47 is a comparison of the data to leading order QCD for a cone size of 1.0 using the structure function MRS set 3 .

A slight excess of events can be observed in these figures for high values of $E_{t}$ If quarks are composite, then this excess is what would be expected to be observed. This excess will be examined in more detail in the following section.

2. A Compusitc Model

The cross section calculated using a Composite Model agrees with QCD below $\sim 160 \mathrm{GeV}$, above this compositeness deviates from $\mathrm{QCD}$, this can be seen in 
Table XXX1. Combined $\chi^{2}$ (fit region combined with upper region), number of l,ins (including the empty bin) and the confidence level obtained using the various structure functions for a cone size of $\mathbf{0 . 4}$.

\begin{tabular}{|c|c|c|c|}
\hline \multicolumn{4}{|l|}{ Structure } \\
\hline Function & $x^{2}$ & $\mathrm{NoB}$ & C.L. (\%) \\
\hline DO 1 & 27.85 & 29 & 52.6 \\
\hline DO 2 & 35.58 & 29 & 18.6 \\
\hline EIILQ 1 & 46.59 & 29 & 2.0 \\
\hline EIILQ 2 & 66.74 & 29 & 0.0 \\
\hline DFLM 1 & 68.59 & 29 & 0.0 \\
\hline DFLM 2 & 69.97 & 29 & 0.0 \\
\hline DFLM 3 & 64.54 & 29 & 0.0 \\
\hline MRS I & 29.83 & 29 & 42.3 \\
\hline MRS 2 & 23.46 & 29 & 75.5 \\
\hline MRS 3 & 21.96 & 29 & 82.2 \\
\hline MRS E & 30.80 & 29 & 37.5 \\
\hline MRS B & 24.86 & 29 & 68.6 \\
\hline TM 1 & 36.46 & 29 & 16.1 \\
\hline TM 2 & 36.38 & 29 & 16.3 \\
\hline TM 3 & 22.59 & 29 & 79.5 \\
\hline TM 4 & 35.72 & 29 & 18.2 \\
\hline
\end{tabular}

Table XYYIL Combined ${ }^{2}$ (fit region combined with upper region), number of bins (including the empty bin) and the confidence level obtained using the various structure functions for a cone size of 0.7 .

\begin{tabular}{cccc}
\hline \hline Structure & & & \\
lunction & $\lambda^{2}$ & NoB & C.L. (\%) \\
\hline DO 1 & 22.51 & 31 & 86.6 \\
DO 2 & 83.65 & 31 & 0.0 \\
EIILQ 1 & 17.16 & 31 & 97.9 \\
EILLQ 2 & 29.25 & 31 & 55.6 \\
DFLM 1 & 24.14 & 31 & 80.5 \\
DFLM 2 & 25.78 & 31 & 73.2 \\
DFLM 3 & 24.08 & 31 & 80.7 \\
MRS 1 & 22.03 & 31 & 88.2 \\
MIRS 2 & 30.44 & 31 & 49.4 \\
MIRS 3 & 28.43 & 31 & 59.9 \\
MRS E & 21.69 & 31 & 89.3 \\
MRS B & 33.61 & 31 & 34.2 \\
TM 1 & 14.76 & 31 & 99.4 \\
TM 2 & 14.62 & 31 & 99.4 \\
TM 3 & 25.19 & 31 & 75.9 \\
TM 4 & 15.27 & 31 & 99.2 \\
\hline
\end{tabular}


Table XXXI11, Combined $\chi^{2}$ (fit region combined with upper region), number of bins (including the empty bin) and the confidence level obtained using the various structure functions for a conc size of 1.0 .

\begin{tabular}{cccc}
\hline \hline Structure & & \\
Function & $x^{2}$ & NoB & C.L. (\%) \\
\hline DO 1 & 25.87 & 30 & 68.2 \\
DO 2 & 63.91 & 30 & 0.0 \\
EILQ 1 & 29.79 & 30 & 47.7 \\
EIILQ 2 & 41.02 & 30 & 8.7 \\
DFLM 1 & 41.44 & 30 & 8.0 \\
DFLM 2 & 44.20 & 30 & 4.6 \\
DFLM 3 & 42.81 & 30 & 6.1 \\
MRS 1 & 26.88 & 30 & 62.9 \\
MRS 2 & 36.32 & 30 & 19.8 \\
MRS 3 & 35.87 & 30 & 21.2 \\
MRS E & 25.77 & 30 & 68.7 \\
MRS B & 36.29 & 30 & 19.9 \\
TM 1 & 27.57 & 30 & 59.3 \\
TM 2 & 27.72 & 30 & 58.5 \\
TM 3 & 29.83 & 30 & 47.5 \\
TM 4 & 23.78 & 30 & 78.2 \\
\hline \hline
\end{tabular}



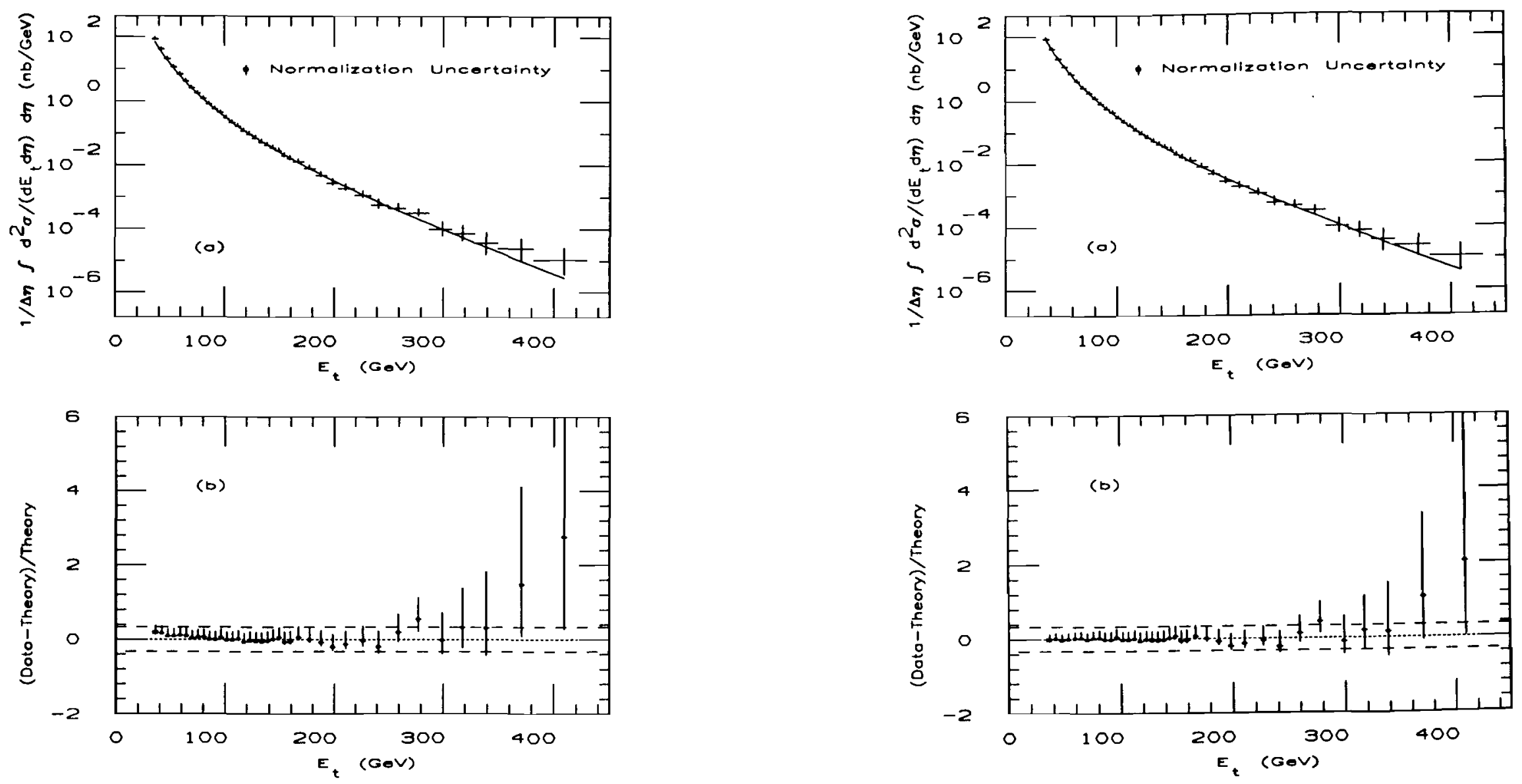

Figure 42. Mcasured cross section compared to leading order QCD using the structure function MRS set $B$ and a cone size of 0.4 . Shown is the a) cross section and b) fractional difference of data with theory.

Figure 43. Measured cross section compared to leading order QCD using the structure function MRS set 3 and a cone size of 0.4. Shown is the a) cross section and b) fractional difference of data with theory. 

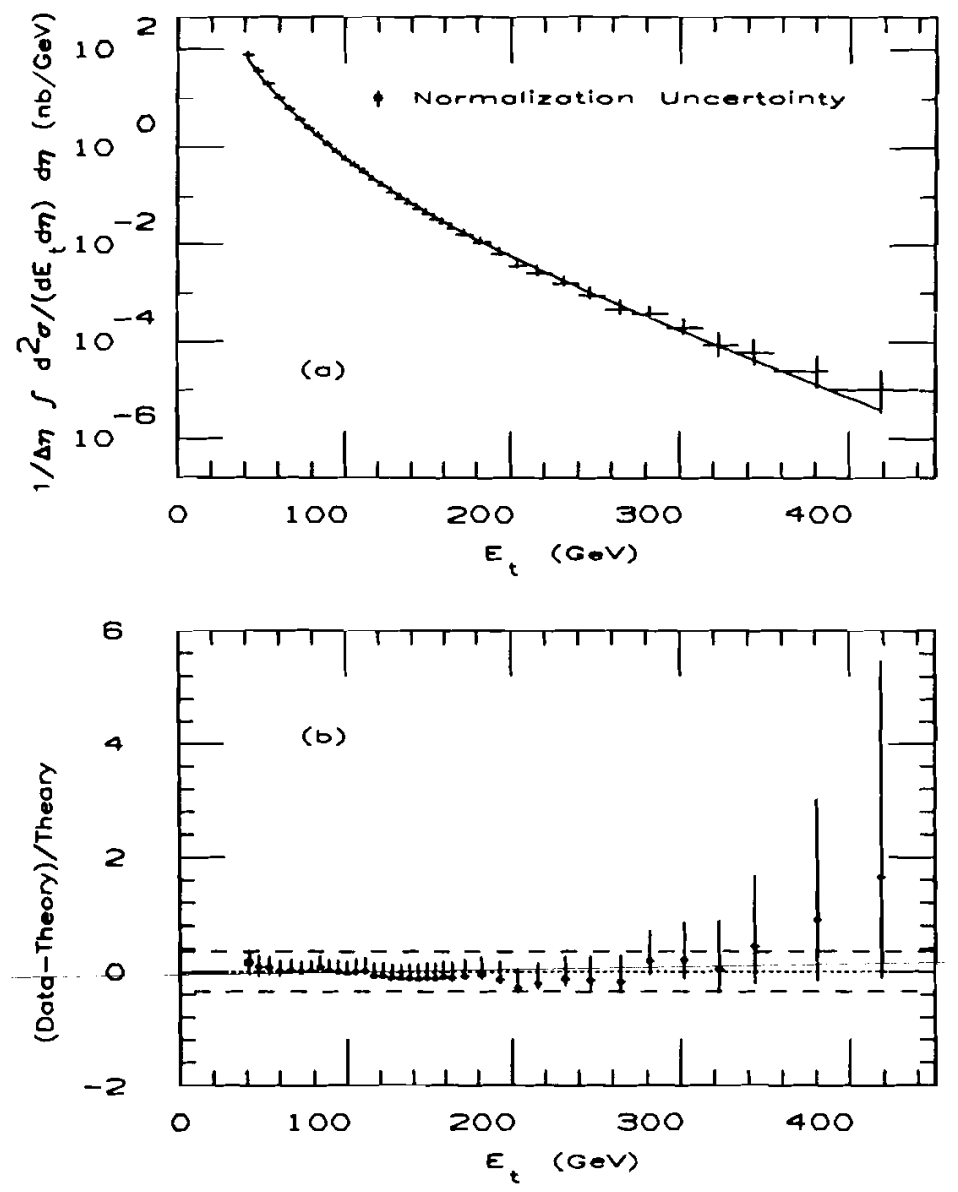

Figure 44. Measured cross section compared to leading order $Q C D$ using the structure function MRS set $B$ and a cone size of 0.7. Shown is the a) cross section and b) fractional difference of data with theory.
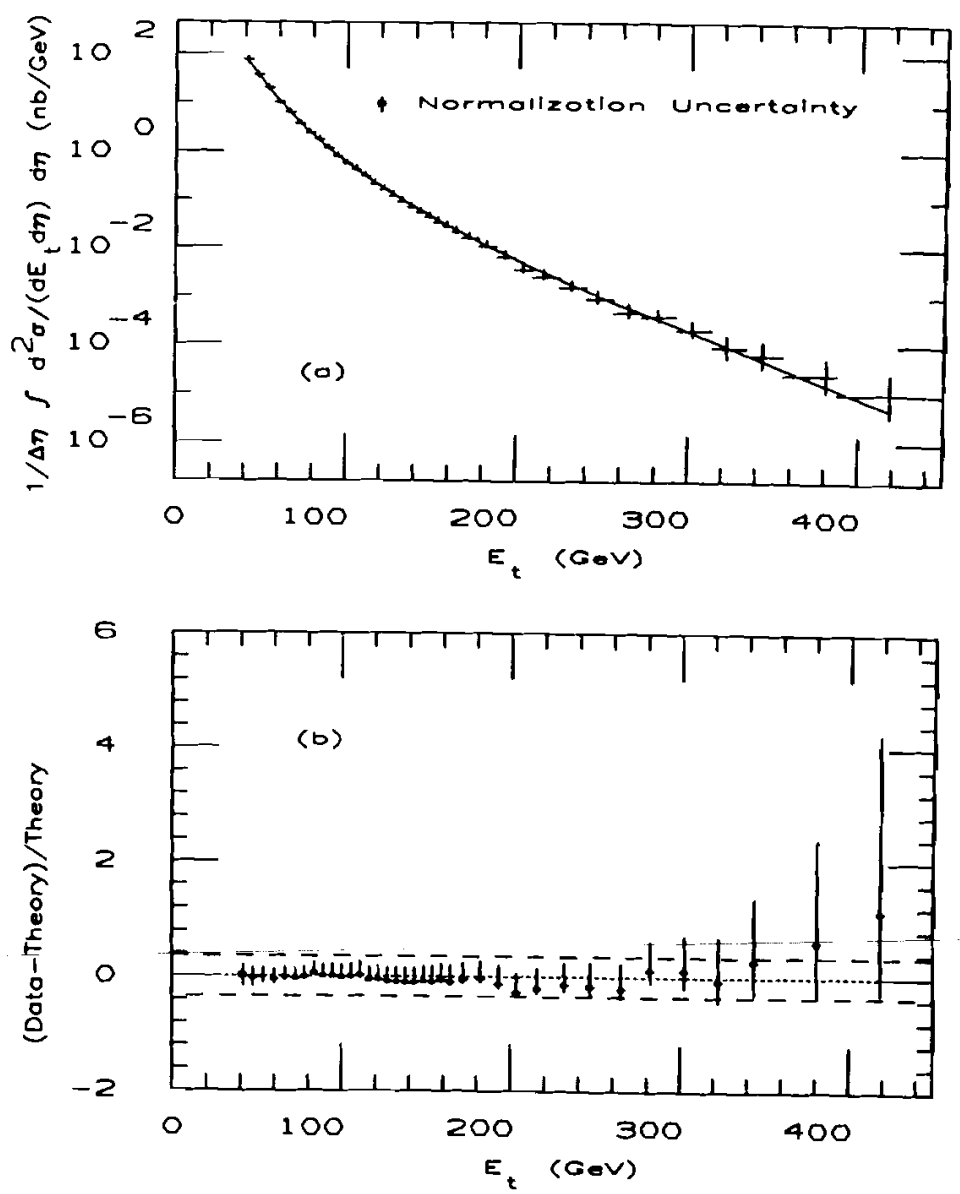

Figure 45. Measured cross section compared to leading order QCD using the structure function MRS set 3 and a cone size of 0.7. Shown is the a) cross section and $b$ ) fractional difference of data with theory 

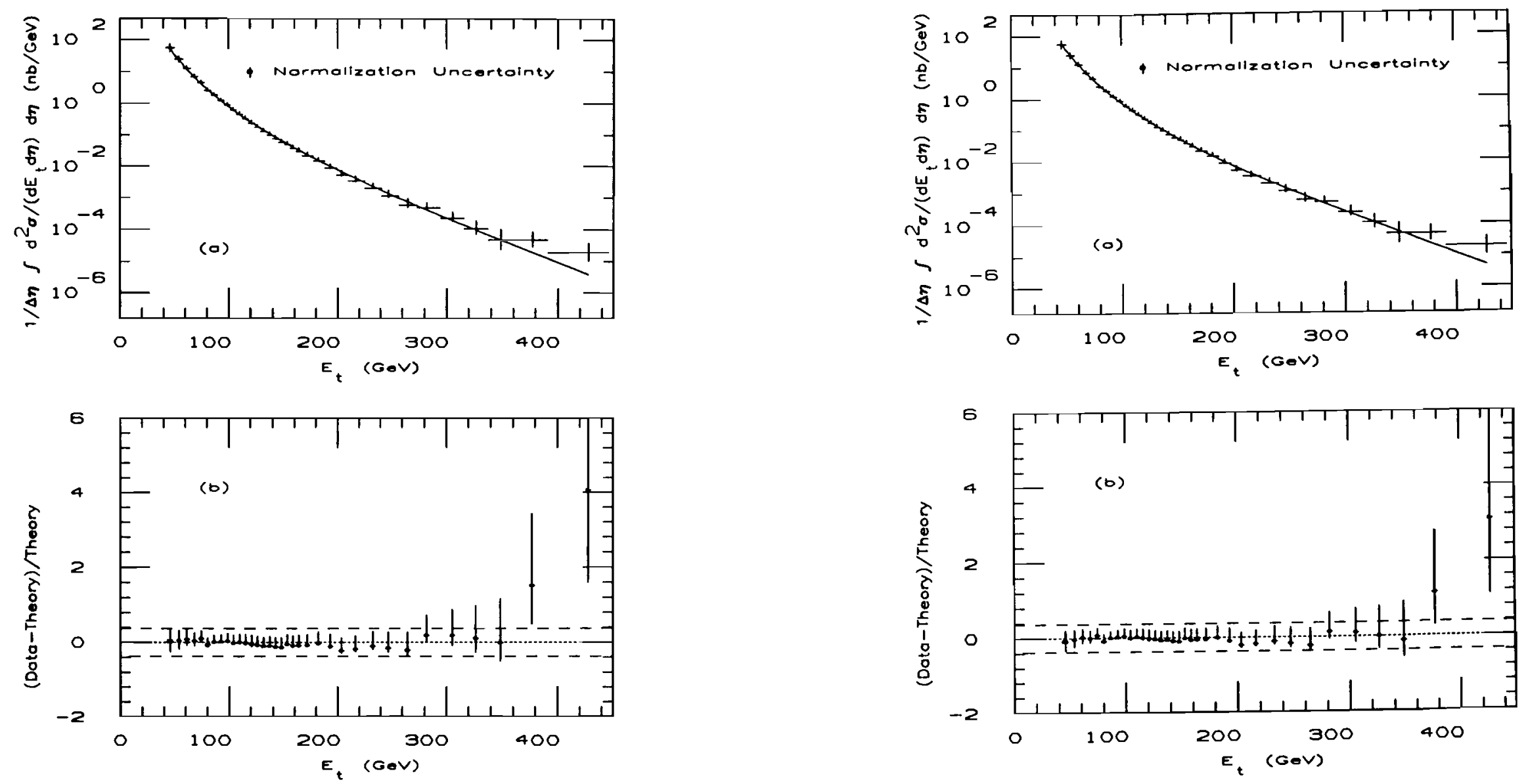

Figure 46. Measured cross section compared to leading order QCD using the structure function MRS set $\mathbf{B}$ and a cone size of 1.0. Shown is the a) cross section and b) fractional difference of data with theory.

Figure 47. Measured cross section compared to leading order QCD using the structure function MRS set 3 and a cone size of 1.0. Shown is the a) cross section and $b$ ) fractional difference of data with theory. 
Figure 41 when using the structure function DFLM set 1 . Fitting the composite calculation of the cross section to the measured cross section below this limit therefore allows the upper region to be examined in order to search for any possible composite signal. If no signal is observed, the confidence level calculated in the upper region allows a lower limit on the value of the conposite scale parasucter $\Lambda_{c}$ to be set.

Ising the structure functions which jieldad wufidence ieveis of more than $5 \%$ in the fitted region for QCD, the measured cross section has been fit to the cross section calculated for a Composite Model. The confidence levels calculated in the fit region and the upper region for the various values of $\Lambda_{c}$ used in the Composite Model are listed in Table XXXIV for a cone size of 0.4 , Table XXXV for a cone size of 0.7 , and Table XXXVI for a cone size of 1.0.

Figurc 48 shows the measured cross section compared to the composite calculation $\left(\Lambda_{c}=980 \mathrm{GeV}\right)$ on both a linear and logarithmic scale using structure function MIRS set 2 for a cone size of 0.4. Figure 49 shows the same comparison for a value of $\Lambda_{c}=1010 \mathrm{GeV}$. Figure 50 shows the measured cross section compared to the compesite ealeulation $\left(\mathrm{A}_{c}=1050 \mathrm{GeV}\right)$ on both a thicar and logarithmic scale using structure function EIILQ set 2 for a cone size of 0.7 . Figure 51 shows the same comparison for a value of $\Lambda_{c}=1090 \mathrm{GeV}$. Figure 52 shows the measured cross section compared to the composite calculation $\left(A_{c}=1100 \mathrm{GeV}\right)$ on both a linear and logarithmic scale using structure function EIIL,Q set 2 for a cone size of 1.0 . Figure 53 shows this comparison for a value of $\Lambda_{c}=1130 \mathrm{GeV}$ using the structure function DFLA1 set 3 for the same cone size.
Table XXXIV. Confidence Levels (\%) in the fit region and upper region for a cone size of 0.4 using the various structure functions.

\begin{tabular}{|c|c|c|c|c|c|c|c|c|}
\hline $\begin{array}{l}\text { Structure } \\
\text { Function }\end{array}$ & $\begin{array}{l}\Lambda_{c}= \\
\text { Fit }\end{array}$ & $\begin{array}{c}950 \mathrm{GeV} \\
\mathrm{U}_{\text {pper }}\end{array}$ & $\begin{array}{l}\Lambda_{c}= \\
\text { Fit }\end{array}$ & $\begin{array}{c}000 \mathrm{GeV} \\
\text { Upper }\end{array}$ & $\begin{array}{l}\Lambda_{c}= \\
\text { Fit }\end{array}$ & $\begin{array}{c}1050 \mathrm{Gc} \\
\text { Upper }\end{array}$ & $\begin{array}{l}\Lambda_{c}= \\
\text { Fit }\end{array}$ & $\begin{array}{c}1100 \mathrm{GeV} \\
\text { Upper }\end{array}$ \\
\hline DO 1 & 75.4 & 0.0 & 71.4 & 0.0 & 67.2 & 0.2 & 63.6 & 1.7 \\
\hline DO 2 & 4.4 & 0.0 & 42.3 & 0.0 & 43.4 & 0.0 & 44.2 & 0.0 \\
\hline EILLQ 1 & 19.7 & 0.0 & 16.2 & 0.1 & 13.3 & 0.8 & 12.7 & 3.1 \\
\hline EIII,Q 2 & 4.0 & 0.0 & 3.4 & 0.0 & 3.1 & 0.0 & 3.0 & 0.0 \\
\hline DFLM I & 2.2 & 0.0 & 1.7 & 0.0 & 1.5 & 0.0 & 1.3 & 0.0 \\
\hline DFLM 2 & 2.0 & 0.0 & 1.7 & 0.0 & 1.5 & 0.0 & 1.3 & 0.0 \\
\hline DFLM 3 & 3.6 & 0.0 & 3.1 & 0.0 & 2.8 & 0.0 & 2.5 & 0.0 \\
\hline MRS I & 76.2 & 0.0 & 69.9 & 0.0 & 64.9 & 0.1 & 61.4 & 0.7 \\
\hline MARS 2 & 76.7 & 1.2 & 76.9 & 7.3 & 77.4 & 18.4 & 77.1 & 32.5 \\
\hline MHS 3 & 87.5 & 0.0 & 87.9 & 0.0 & 87.9 & 0.1 & 87.8 & 1.1 \\
\hline MRS E & 55.2 & 0.0 & 64.9 & 0.0 & 45.9 & 0.0 & 42.7 & 1.7 \\
\hline MRS B & 64.6 & 0.1 & 69.6 & 1.1 & 70.5 & 7.1 & 70.9 & 17.7 \\
\hline $\mathrm{TM} 1$ & 31.4 & 0.0 & 24.9 & 0.0 & 21.6 & 0.0 & 19.1 & 0.0 \\
\hline T'M 2 & 31.2 & 0.0 & 26.6 & 0.0 & 22.6 & 0.0 & 20.7 & 0.1 \\
\hline TM 3 & 88.5 & 0.0 & 84.8 & 0.0 & 82.0 & 0.0 & 79.0 & 0.0 \\
\hline TM 4 & $\overline{\mathrm{3} 3.2}$ & 0.0 & 27.5 & 0.0 & 25.3 & 0.0 & 23.7 & 0.0 \\
\hline
\end{tabular}


Table XXXV. Confidence levels (\%) in the fit region ant upper region for a conc sizc of 0.7 using the various structure functions.

\begin{tabular}{|c|c|c|c|c|c|c|c|c|}
\hline $\begin{array}{c}\text { Structure } \\
\text { Function } \\
\end{array}$ & $\begin{array}{l}\Lambda_{c}= \\
\text { Fit }\end{array}$ & $\begin{array}{c}1000 \mathrm{GeV} \\
\text { Upper }\end{array}$ & $\begin{array}{l}\Lambda_{c}= \\
\text { Fit }\end{array}$ & $\begin{array}{c}1050 \mathrm{GeV} \\
\text { Upper }\end{array}$ & $\begin{array}{l}\Lambda_{c}= \\
\text { Yit }\end{array}$ & $\begin{array}{c}1100 \mathrm{GeV} \\
\text { Upper }\end{array}$ & $\begin{array}{l}\Lambda_{c}= \\
\text { Fit }\end{array}$ & $\begin{array}{c}1150 \mathrm{GeV} \\
\text { Upper }\end{array}$ \\
\hline DO 1 & 93.8 & 0.0 & 94.1 & 0.0 & 94.3 & 0.0 & 94.4 & 0.0 \\
\hline DO 2 & 1.9 & 0.0 & 1.9 & 0.0 & 2.0 & 0.0 & 2.0 & 0.0 \\
\hline EIILQ 1 & 99.7 & 0.0 & 99.6 & 0.0 & 99.6 & 0.9 & 99.7 & 6.7 \\
\hline EHLQ 2 & 99.8 & 0.4 & 99.8 & 4.3 & 99.8 & 12.4 & 99.8 & 23.0 \\
\hline DFLM 1 & 99.5 & 0.0 & 99.4 & 0.0 & 99.3 & 0.0 & 99.1 & 0.5 \\
\hline DFIM 2 & 99.6 & 0.0 & 99.4 & 0.2 & 99.2 & 1.9 & 99.1 & 7.3 \\
\hline DFLM 3 & 99.9 & 0.3 & 99.9 & 2.7 & 99.9 & 10.7 & 99.9 & 21.6 \\
\hline MRS 1 & 95.8 & 0.0 & 96.1 & 0.0 & 96.2 & 0.0 & 96.3 & 0.0 \\
\hline MRS 2 & 25.6 & 0.0 & 27.3 & 0.2 & 28.0 & 1.6 & 29.0 & 6.1 \\
\hline MRS 3 & 66.0 & 0.0 & 63.8 & 0.0 & 61.3 & 0.0 & 60.8 & 0.0 \\
\hline MRS E & 96.9 & 0.0 & 97.2 & 0.0 & 97.1 & 0.0 & 97.1 & 0.0 \\
\hline MRS B & 17.4 & 0.0 & 15.8 & 0.0 & 11.5 & 0.0 & 18.4 & 1.4 \\
\hline TM I & 99.6 & 0.0 & 99.6 & 0.0 & 99.5 & 0.0 & 99.7 & 0.0 \\
\hline TM 2 & 99.7 & 0.0 & 99.6 & 0.0 & 99.6 & 0.0 & 99.7 & 0.0 \\
\hline TM 3 & 96.1 & 0.0 & 96.9 & 0.0 & 97.4 & 0.0 & 97.6 & 0.0 \\
\hline TM 4 & 99.5 & 0.0 & 99.4 & 0.0 & 99.3 & 0.0 & 99.2 & 0.0 \\
\hline
\end{tabular}

Table XXXVI. Confidence Levels $(\%)$ in the fit region and upper region for a cone size of 1.0 using the various structure functions.

\begin{tabular}{|c|c|c|c|c|c|c|c|c|}
\hline $\begin{array}{l}\text { Siructure } \\
\text { Function }\end{array}$ & $\begin{array}{l}\Lambda_{c}= \\
\text { Pit }\end{array}$ & $\begin{array}{c}1050 \mathrm{GcV} \\
\text { Upper }\end{array}$ & $\begin{array}{l}\Lambda_{c}= \\
\text { Fil }\end{array}$ & $\begin{array}{c}1100 \mathrm{GeV} \\
\text { Upper }\end{array}$ & $\begin{array}{l}\Lambda_{c}= \\
\mathrm{Fit}\end{array}$ & $\begin{array}{c}1150 \mathrm{GeV} \\
\text { Upper }\end{array}$ & $\begin{array}{l}\Lambda_{c}= \\
\text { Fit }\end{array}$ & $\begin{array}{c}1200 \mathrm{GeV} \\
\text { Upper }\end{array}$ \\
\hline DO 1 & 94.9 & 0.0 & 95.4 & 0.0 & 95.7 & 0.0 & 95.7 & 0.0 \\
\hline DO 2 & 6.0 & 0.0 & 6.3 & 0.0 & 6.5 & 0.0 & 6.4 & 0.0 \\
\hline EHLQ I & 96.5 & 0.0 & 96.4 & 0.2 & 97.0 & 1.6 & 97.1 & 6.3 \\
\hline EIILQ 2 & 90.5 & 1.2 & 88.9 & 4.6 & 87.9 & 9.7 & 86.8 & 12.1 \\
\hline DPI,M I & 51.1 & 0.0 & 36.2 & 0.0 & 39.5 & 0.0 & 35.1 & 0.1 \\
\hline 1)FLM 2 & 17.5 & 0.0 & 41.1 & 0.1 & 37.0 & 0.3 & 30.1 & 10.8 \\
\hline 1)FLM 3 & 734 & 0.4 & 70.4 & 1.4 & 65.4 & 17.1 & 65.9 & 16.6 \\
\hline MIRS 1 & 95.5 & 0.0 & 96.0 & 0.0 & 96.2 & 0.0 & 96.3 & 0.0 \\
\hline MIRS 2 & 49.1 & 0.0 & 49.8 & 0.6 & 50.1 & 2.9 & 50.4 & 7.2 \\
\hline MIRS 3 & 50.4 & 0.0 & 51.1 & 0.0 & 52.0 & 0.0 & 52.3 & 0.0 \\
\hline MILS E & 95.8 & 0.0 & 96.1 & 0.0 & 96.3 & 0.0 & 96.2 & 0.0 \\
\hline MRS B & 40.9 & 0.0 & 41.8 & 0.0 & 43.0 & 0.3 & 43.3 & 1.7 \\
\hline ГМ I & 96.8 & 0.0 & 96.6 & 0.0 & 96.5 & 0.0 & 96.2 & 0.0 \\
\hline TM 2 & 96.7 & 0.0 & 96.3 & 0.0 & 96.2 & 0.0 & 95.7 & 0.0 \\
\hline TM 3 & 85.9 & 0.0 & 86.9 & 0.0 & 90.5 & 0.0 & 91.3 & 0.0 \\
\hline ГМ 4 & 97.4 & 0.0 & 97.4 & 0.0 & 97.1 & 0.0 & 96.9 & 0.0 \\
\hline
\end{tabular}



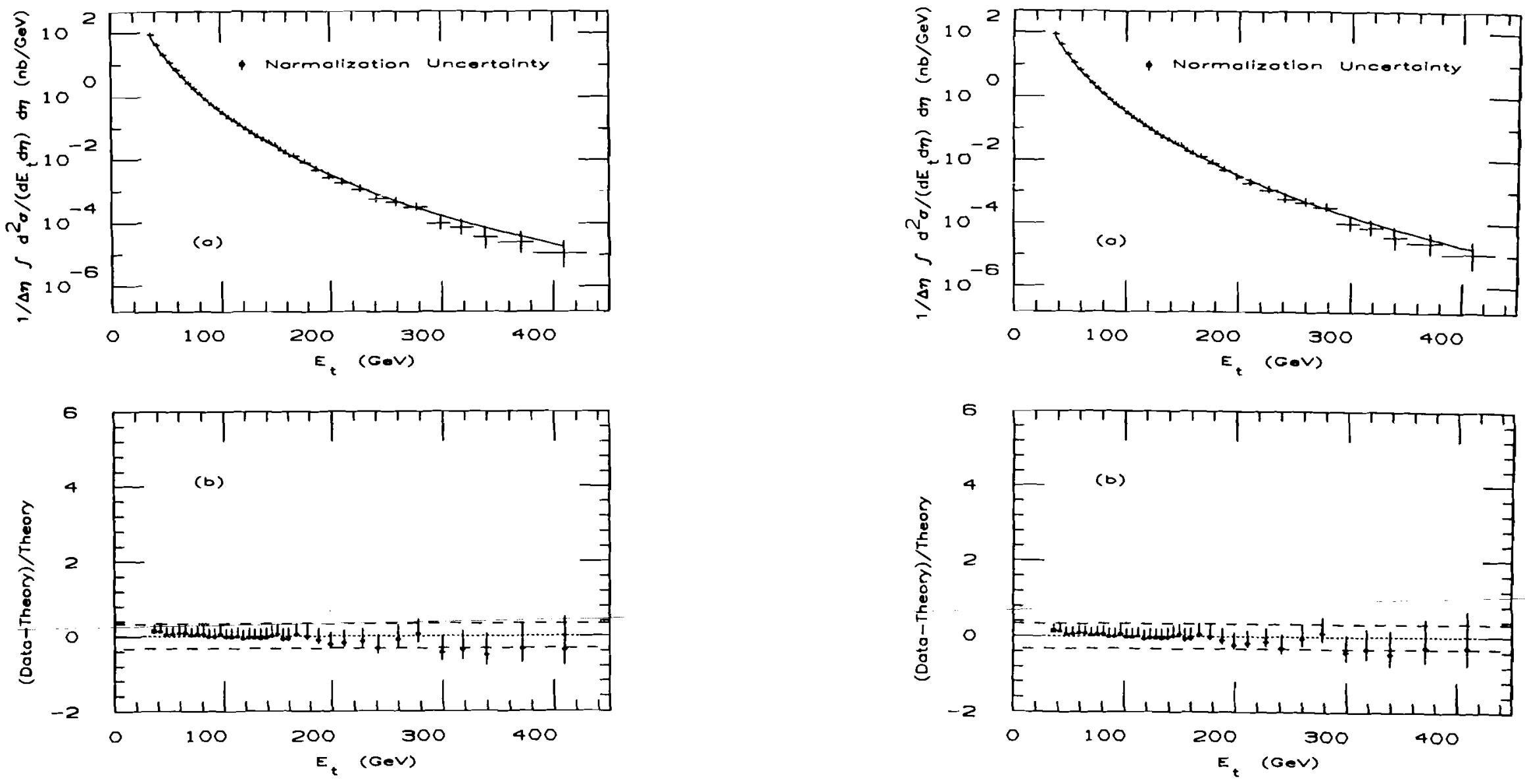

Figure 48. Composite calculation $\left(\Lambda_{c}=980 \mathrm{GcV}\right)$ of the inclusive jet cross sec(ion compare to the measure cross section using the structure function MRS set 2 and a cone size of 0.4 . Shown is the a) cross section and b) fractional difference of data witls theory.

Figure 40. Composite calculation $\left(\Lambda_{c}=1010 \mathrm{GeV}\right)$ of the inclusive jet cross section conpare to the measure cross section using the structure function MIRS set 2 and a cone size of 0.4. Shown is the a) cross section and b) Iractional difference of data with theory. 

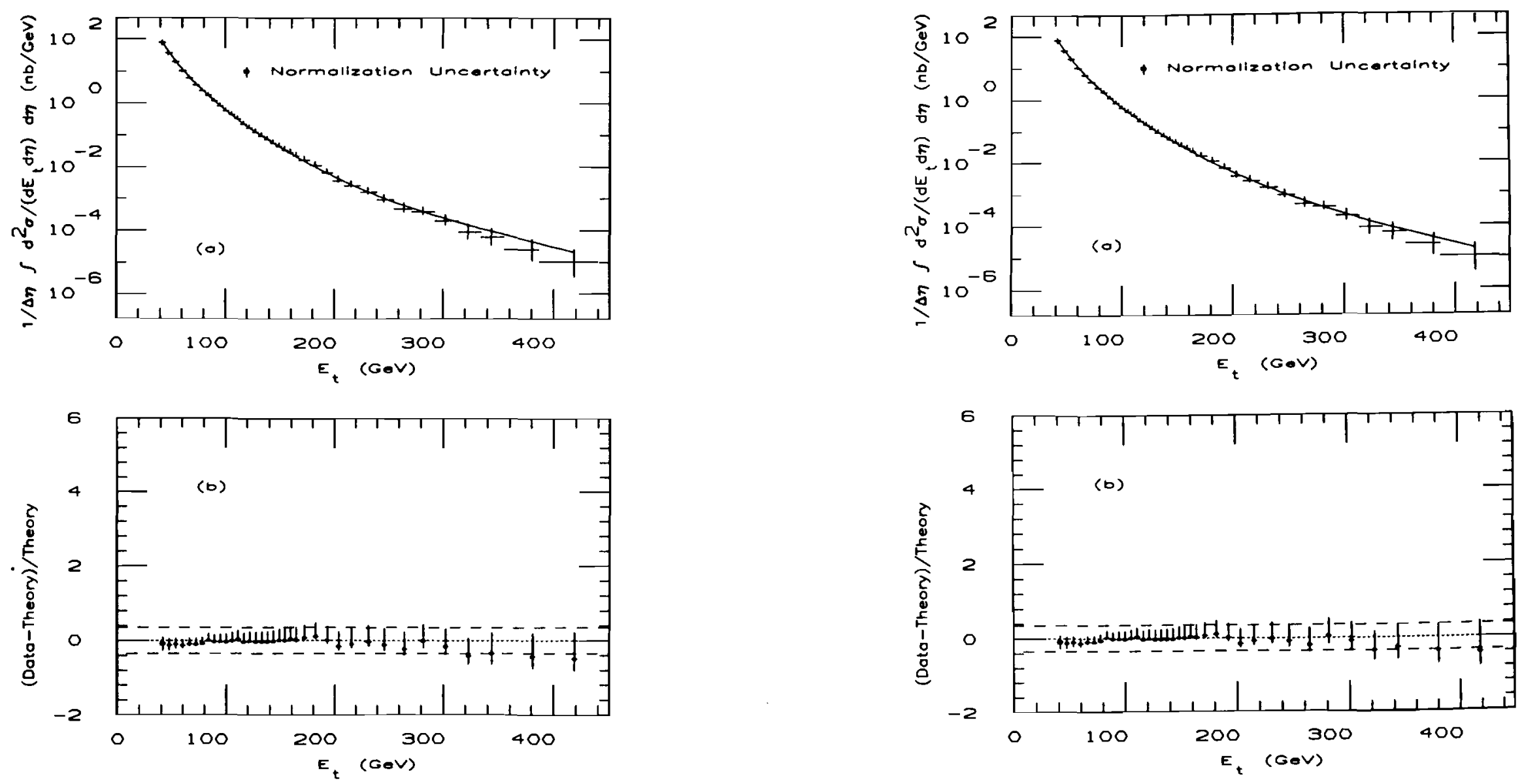

Figure 50. Composite calculation $\left(\Lambda_{c}=1050 \mathrm{GeV}\right)$ of the inclusive jet cross section compare to the measure cross section using the structure function FIILQ set 2 and a cone size of 0.7 . Shown is the a) cross section and b) fractional difference of data with theory.

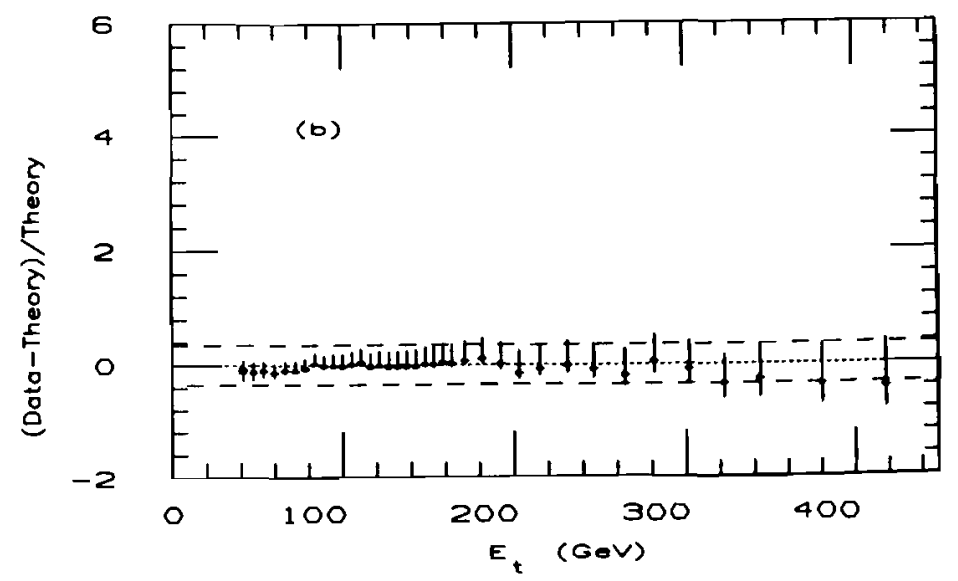

Figure 51. Composite calculation $\left(\Lambda_{c}=1090 \mathrm{GeV}\right)$ of the inclusive jet cross sec tion compare to the measure cross section using the structure function EulQ set 2 and a cone size of 0.7 . Shown is the a) cross section and b) fractional difference of data with theory. 

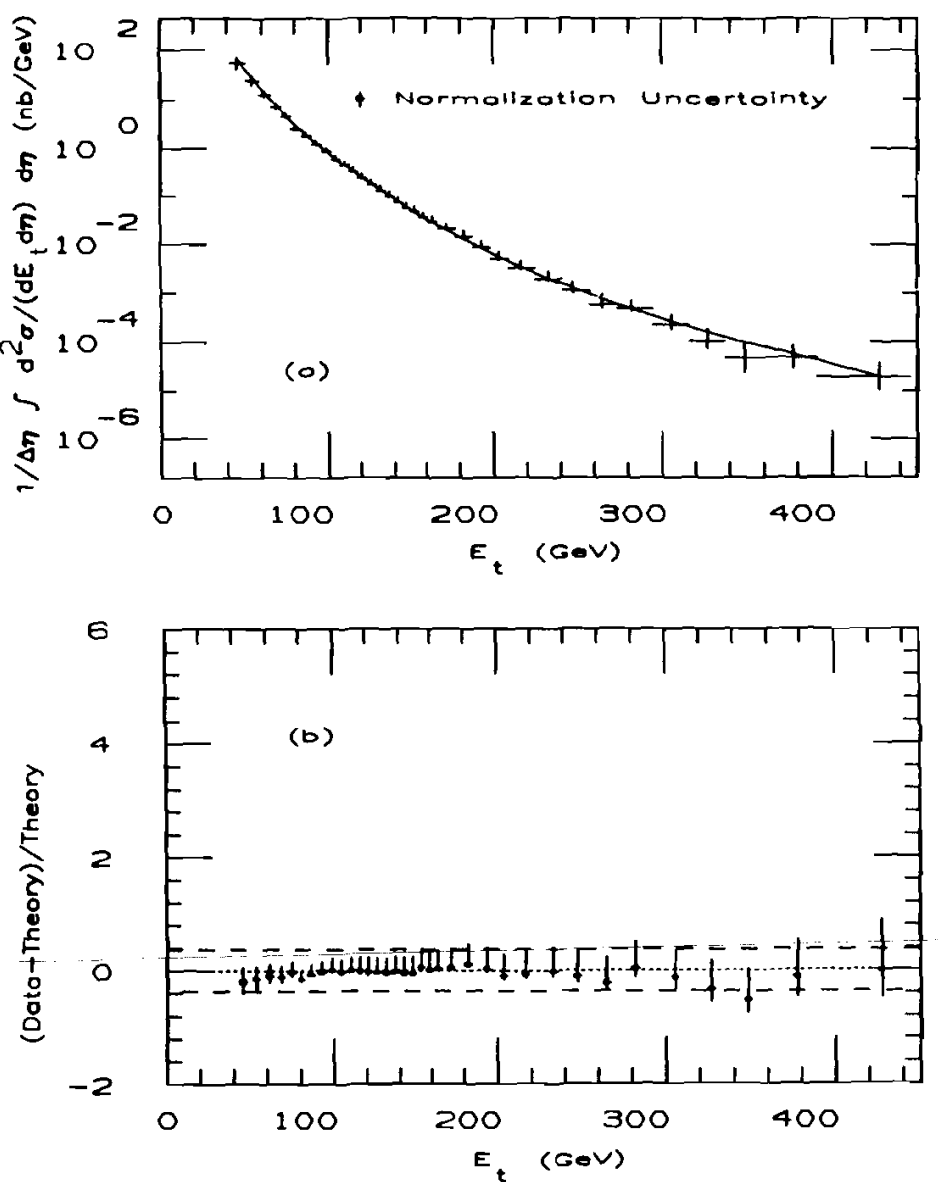

Figure 52. Composite calculation $\left(\Lambda_{c}=1100 \mathrm{GeV}\right)$ of the inclusive jet cross section compare to the measure cross section using the structure function EIILQ set 2 and a cone size of 1.0. Shown is the a) cross section and b) fractional difference of data with theory.
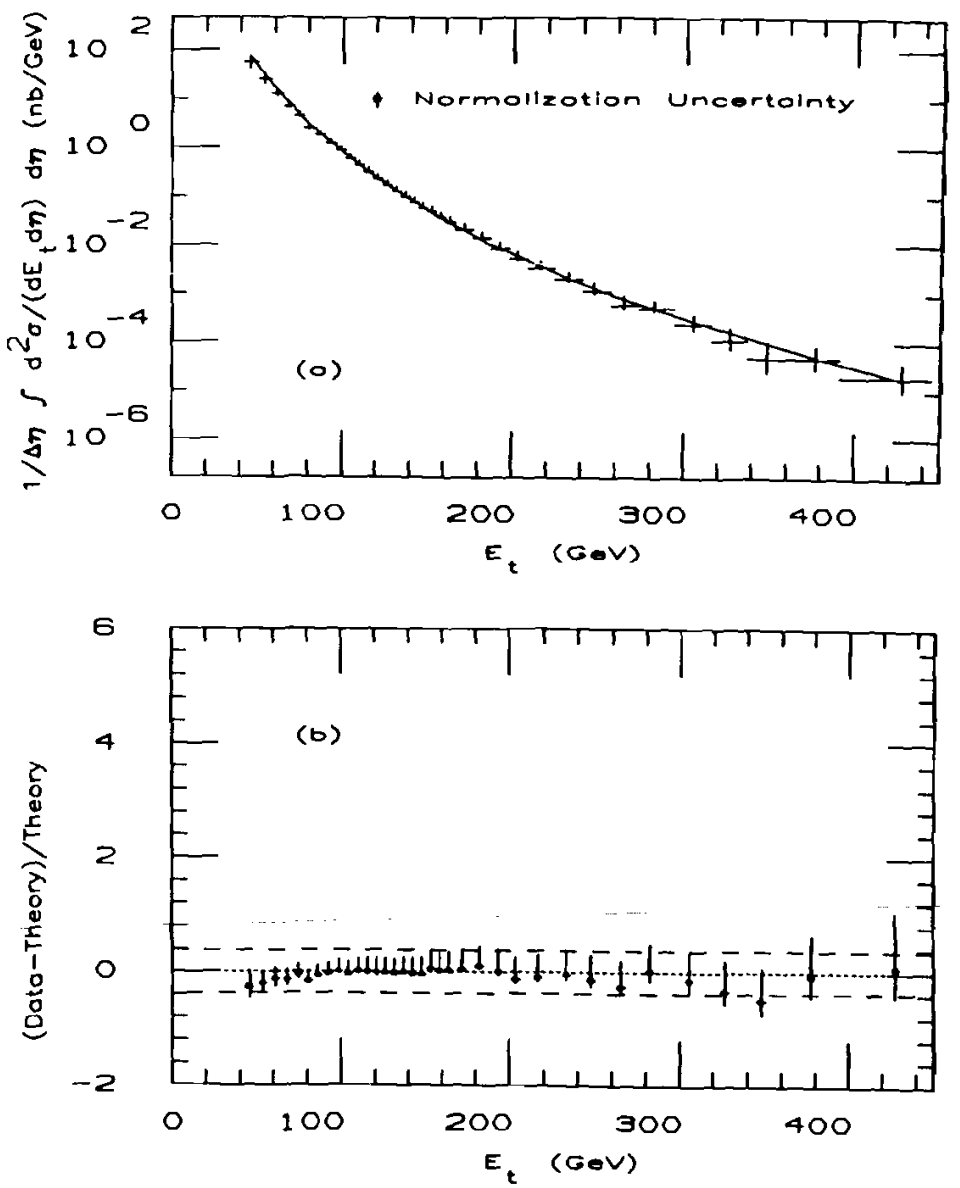

Figure 53. Composite calculation $\left(\Lambda_{c}=1130 \mathrm{GeV}\right)$ of the inclusive jet cross section compare to the measure cross section using the structure function DFI.M set 3 and a cone size of 1.0 . Shown is the a) cross section and b) fractional difference of data with theory. 
The slight excess of events observed in Figures $42,43,44,45,46$, and 47 may indicate the possibility of new plysics. Using the normalization obtained in the fit region and the corrections obtained for the data, the number of jets expected to be observed for QCD and Compositeness can be calculated. The difference in the number of jets predicted and the number of jets observed divided by the square root of the number of predicted jets is a measure of the significance of this excess. Tables XXXVII, XXXVIII, XXXIX list the number of jets found in the region of excess and the number of jets predicted by varions theoretical models for the different cone sizes. The significance is also calculated and listed in these tables. As shown in Tables XXXVII, XXXVIII, XXXIX the significance of this excess is on the level of 1-3 sigma, depending on the choice of structure function, and therefore its statistical significance is questionable.

As shown in Table XL, the number of jets and the number of events per bin is nearly the same in the last six bins of the data (where the excess is observed) for each cone size. Thus, the effect of the statistical uncertainty, due to the counting of independent events, on the fitting procedure is small. As the systematic uncertainty is large for these bins, this statistical effect was ignored in the fitting procedure. The total number of jets is slightly larger than the total number of events in the last six bins. This is duc to the fact that in most cases if there is a second jet in the event contributing to the cross section, then these two jets nearly balance in $E_{t}$. This has a small effect on the calculation of the significance mentioned in the previous paragraph. It also suggests that the number of events in the data may be compared
Table XXXVII. Number of jets found in the $E_{t}$ range from 266.0 to $429.6 \mathrm{GeV}$, number of jets predicted by theory and the significance of this excess for a cone size of $\mathbf{0 . 4}$.

\begin{tabular}{cccc}
\hline \hline \multirow{2}{*}{ Structure } & \multicolumn{3}{c}{ Number of Jets } \\
Function & Data & Theory & Significance \\
\hline DO 1 & 48 & 39.2 & -1.40 \\
DO 2 & 48 & 50.8 & 0.39 \\
ELILQ 1 & 48 & 33.8 & -2.44 \\
EHLQ 2 & 48 & 27.0 & -4.03 \\
DFLM 1 & 48 & 29.4 & -3.43 \\
DFLM 2 & 48 & 28.1 & -3.76 \\
DFLM 3 & 48 & 28.5 & -3.66 \\
MRS 1 & 48 & 38.0 & -1.63 \\
MRS 2 & 48 & 36.6 & -1.89 \\
MRS 3 & 48 & 41.0 & -1.09 \\
MRS E & 48 & 37.7 & -1.67 \\
MRS B & 48 & 37.5 & -1.72 \\
TM 1 & 48 & 39.8 & -1.29 \\
TM 2 & 48 & 40.0 & -1.27 \\
TM 3 & 48 & 44.7 & -0.49 \\
TM 4 & 48 & 39.9 & -1.28 \\
\hline \hline
\end{tabular}


Table XXXVIII. Number of jets found in the $E_{t}$ range from 271.9 to $410.0 \mathrm{GeV}$, number of jets predicted by theory and the significance of this excess lor a cone size of 0.7 .

\begin{tabular}{cccc}
\hline Structure & \multicolumn{3}{c}{ Number of Jets } \\
Function & Data & Theory & Significance \\
\hline DO 1 & 64 & 58.9 & -0.67 \\
DO 2 & 64 & 135.7 & 6.16 \\
EILQ 1 & 64 & 49.7 & -2.02 \\
EILQ 2 & 64 & 42.7 & -3.26 \\
DFLM 1 & 64 & 48.2 & -2.28 \\
DFLM 2 & 64 & 45.7 & -2.70 \\
DFLM 3 & 64 & 45.1 & -2.82 \\
MIRS 1 & 64 & 56.8 & -0.96 \\
MRS 2 & 64 & 57.8 & -0.82 \\
MRS 3 & 64 & 60.1 & -0.50 \\
MRS E & 64 & 56.5 & -1.00 \\
MRS B & 64 & 55.5 & -1.14 \\
TM 1 & 64 & 69.5 & 0.66 \\
TM 2 & 64 & 69.8 & 0.69 \\
TM 3 & 64 & 80.7 & 1.86 \\
TM 4 & 64 & 69.7 & 0.68 \\
\hline \hline
\end{tabular}

Table XXXIX. Number of jets found in the $E_{t}$ range from 273.2 to $445.6 \mathrm{GeV}$, number of jets predicted by theory and the significance of this excess for a cone size of 1.0

\begin{tabular}{cccc}
\hline Structure & \multicolumn{3}{c}{ Number of Jets } \\
Function & Data & Theory & Significance \\
\hline DO 1 & 83 & 71.0 & -1.04 \\
DO 2 & 83 & 156.4 & 5.87 \\
FIILQ 1 & 83 & 63.8 & -2.41 \\
EILL 2 & 83 & 57.3 & -3.40 \\
DFLM 1 & 83 & 64.0 & -2.38 \\
DFLM 2 & 83 & 60.7 & -2.86 \\
DFLM 3 & 83 & 59.2 & -3.09 \\
MRS 1 & 83 & 71.3 & -1.38 \\
MRS 2 & 83 & 69.4 & -1.64 \\
MRS 3 & 83 & 77.1 & -0.67 \\
MRS E & 83 & 71.9 & -1.31 \\
MRS B & 83 & 71.5 & -1.36 \\
TM 1 & 83 & 95.5 & 1.28 \\
TM 2 & 83 & 95.8 & 1.31 \\
TM 3 & 83 & 504.7 & 2.12 \\
TM 4 & 83 & 95.7 & 1.29 \\
\hline \hline
\end{tabular}


Table XL. Number of jets over the number of events per bin found in the last six bins of the data. Also listed is the total number of jets over the total number of events summed over the last six bins of thic data. The results are given for jet clustering cone sizes of $0.4,0.7$, and 1.0 .

\begin{tabular}{cccc}
\hline \hline & \multicolumn{3}{c}{ Cone Size } \\
Bin & 0.4 & 0.7 & 1.0 \\
\hline Last-5 & $26 / 23$ & $31 / 28$ & $41 / 37$ \\
Last-4 & $8 / 8$ & $16 / 14$ & $19 / 17$ \\
Last-3 & $6 / 6$ & $7 / 7$ & $9 / 9$ \\
Last-2 & $3 / 3$ & $5 / 5$ & $4 / 4$ \\
Last-1 & $3 / 3$ & $3 / 3$ & $6 / 6$ \\
Last & $2 / 1$ & $2 / 1$ & $4 / 3$ \\
Total & $48 / 40$ & $64 / 55$ & $83 / 69$ \\
\hline \hline
\end{tabular}

to the number of events predicted by QCD (since munber of events is an inclepencient quantity) when calculating the significance of an excess.

Based on the confidence level calculated in the upper region for the various structure functions and various values of $\Lambda_{c}$, a lower linit on the value of the compositeness scale parameter $\Lambda_{c}$ can be set. Using the structure functions which did not yield a poor $\chi^{2}$ in the fit region for QCD and which gave the best confidence level in the upper region when fitting for compositeness, a conservative limit on $\Lambda_{c}$ can set for each cone size. At the $90 \%$ confidence level $\Lambda_{c}>1010 \mathrm{GeV}$ for a cone size of 0.4 , $\Lambda_{c}>1090 \mathrm{GeV}$ for a cone size of 0.7 , and $\Lambda_{c}>1130 \mathrm{GeV}$ for a cone size of 1.0. At the $95 \%$ confidence level $\Lambda_{c}>980 \mathrm{GeV}$ for a cone size of $0.4, \Lambda_{c}>1050 \mathrm{GeV}$ for a cone size of 0.7 , and $\Lambda_{c}>1100 \mathrm{GeV}$ for a cone size of 1.0 .
The increase int the limit that is found for the composite parameter $\Lambda_{c}$ when the cone size is increased is because the correction for encrgy lost outside the clustering cone is not being applied. This causes the $E_{t}$ of the jets to be larger when the cone size is increased, tlus extending the $E_{t}$ range covered by the cross section. The higher

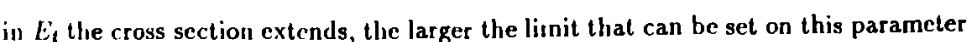
This correction is srnallest for larger cone sizes (since less energy can be lost outside the cone) and thus the limit obtained from the larger cone sizes is more accurate when comparing to a leading order QCD calculation wlich requires this correction to be applicd. Therefore, an absolute limit of $\Lambda_{c}>1100 \mathrm{GeV}$ can be set at the $95 \%$ C.L. based ou a comparison of leading order QCD to the measured jet cross section.

C. Comparisons to Next-to-Leading Order

Comparisons to next-to-leading order QCD calculations of the inclusive jet cross section are done in the same manner as the comparisons are done for leading order. Variations in cross section as a function of cone size can also be investigated when comparing to uext-to-leading order QCD. Both of these comparisons will be examined in this section. Due to the limited availability of computer time and the length of time required to calculate the next-to-leading order QCD cross section, only the structure function MIRS set B could be used to calculate the next-to-leading order QCD cross scction in this analysis, using $Q^{2}=\left(P_{t} / 2\right)^{2}$. Figure 54 slows a comparison of leading 
order QCI) to next-to-teading order QCI) using the structure function MHS set B.

Using this structure function and the same fit regions as described for the leading order fits, comparisons were made to measured inclusive jet cross section for cone sizes of $0.4,0.7$ and 1.0. Table XII lists the $x^{2}$ s, confidence levels and number of bins found in each region for each cone size. Figure 55 shows the agreement between the next-to-leading order QCD calculation and the data for a cone size of 0.4 plotted on both a logarithmic and linear scale. The normalization found for the predicted cross section for a cone size of 0.4 with the data was 0.723 , just within the $33 \%$ normalization uncertainty on the data. Figure 56 shows the agrecment between the next-to-leading order QCD calculation and the data for a cone size of 0.7 plotted on both a logarithmic and linear scale. The normalization found for the predicted cross section for a cone size of 0.7 with the data was 1.03 , well within the $35 \%$ normalization uncertainty on the data. Figure 57 shows the agreement between the next-to-leading order QCD calculation and the data for a conc size of 1.0 plotted on both a logarithmic and linear scale. The normalization found for the predicted cross section for a cone size of 1.0 with the data was 1.07, again well within the $37 \%$ normalization uncertainty on the data.

Anotlicr feature of the next-to-leading order QCD calculations is the predicted variation in cross section as a function of jel clustering cone size. This was examined using cone sizes of $0.4,0.7$ and 1.0. Figure 58 shows the cross section for jets of $E_{t}=100 \mathrm{GeV}$ measured in the data compared to what was calculated using nextto-leading order QCD as a function of the clustering cone size. Figure 58a was fit to $A+B \log (R)$, where $\mathrm{R}$ is the cone size. The result found $A=0.7880 \pm 0.0224$ and $B=0.4923 \pm 0.0264$ for a confidence level of $16.9 \%$, using statistical errors only.
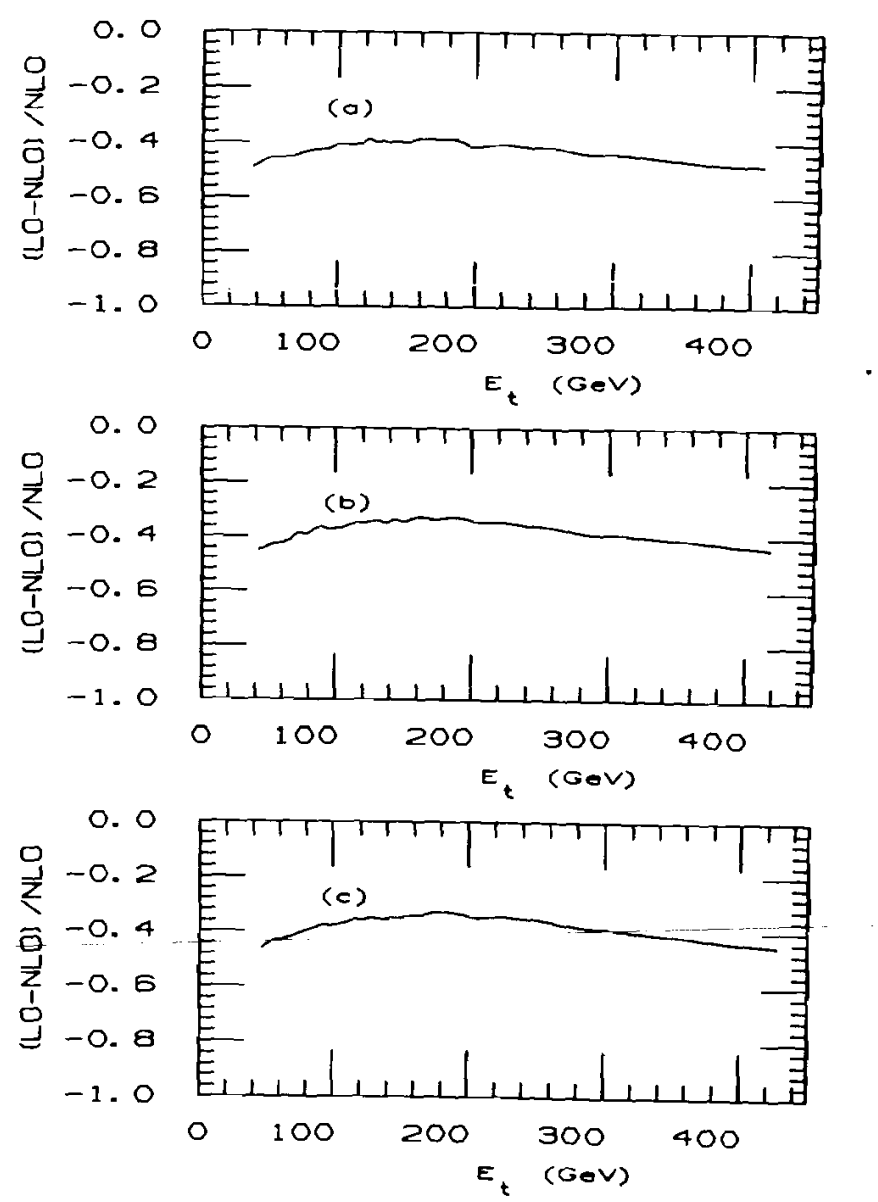

ligure 54. Comparison of next-to-lcading order $Q C D$ and leading order $Q C D$, using structurc function MRS set $B$. Shown are comparisons for cone sizes of a) 0.4, b) 0.7 and c) 1.0. It should also be noted the curves were both normalized separately to the data before being compared. 
Table XII. Results of the comparison of next-to-leading order QCD calculations (using the structure function MIRS B) of the inclusive jet cross section to the measured cross section. The ${ }^{2}$ 's, number of bins and confidence lcvels calculated in each region for each cone size are listed.

\begin{tabular}{cccccccccccc}
\hline \hline & Cone & Size & 0.4 & \multicolumn{2}{c}{ Cone } & Size & 0.7 & \multicolumn{2}{c}{ Cone } & Size & 1.0 \\
Region & $\chi^{2}$ & NoB & C.L. (\%) & $\chi^{2}$ & NoB & C.L. (\%) & $\chi^{2}$ & Nol3 & C.L. (\%) \\
\hline Lower & 6.95 & 10 & 73.1 & 11.59 & 7 & 11.5 & 9.90 & 6 & 12.9 \\
Fit & 8.60 & 14 & 80.2 & 10.70 & 15 & 70.9 & 9.68 & 14 & 71.9 \\
Upper & 10.65 & 15 & 77.7 & 17.06 & 16 & 38.2 & 23.67 & 16 & 9.7 \\
\hline \hline
\end{tabular}
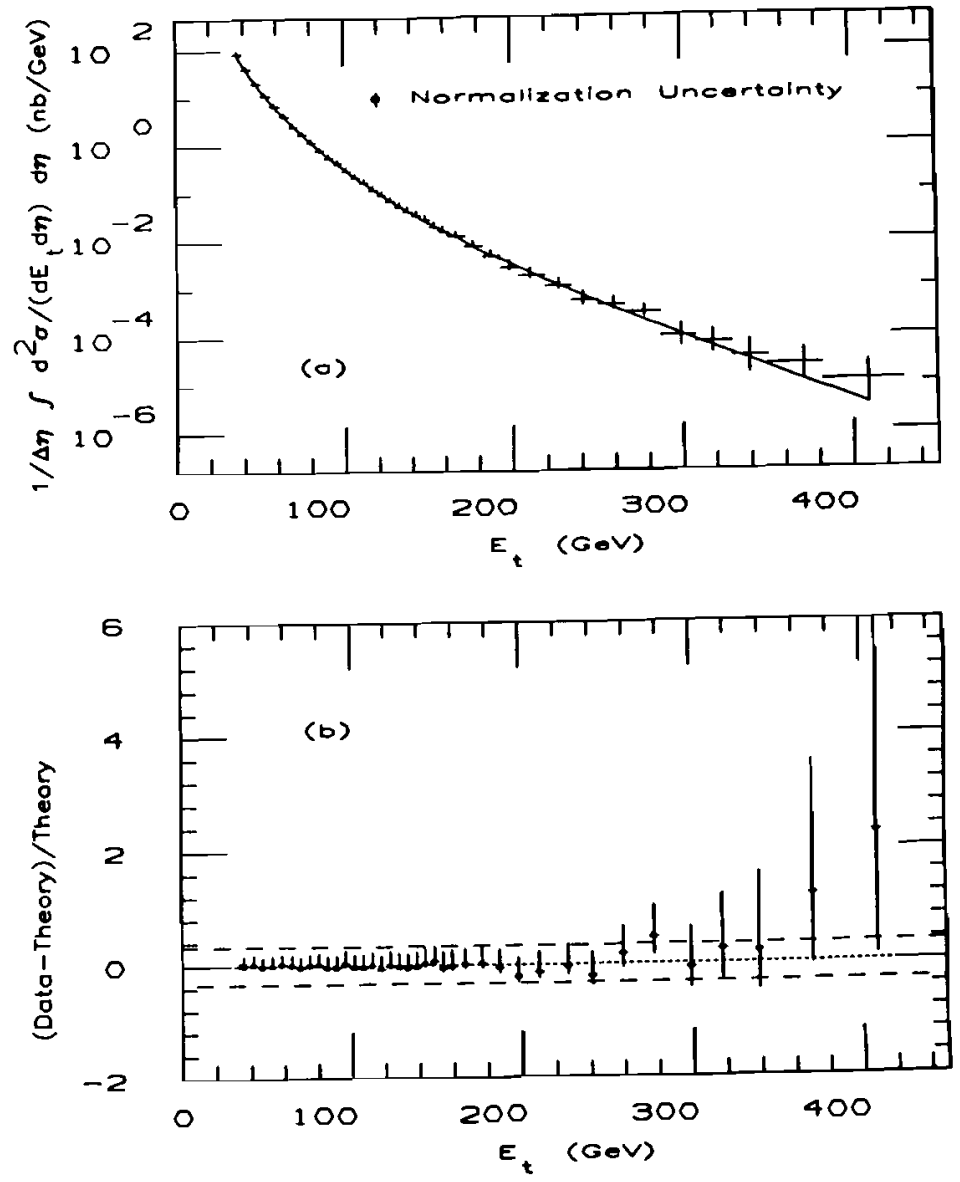

Figure 55. Next-to-leading order QCD calculation of the inclusive jet cross section compare to the measure cross section using the structure function MRS B for a cone size of 0.4 . Shown is the a) cross section and b) fractional difference of data with theory. 

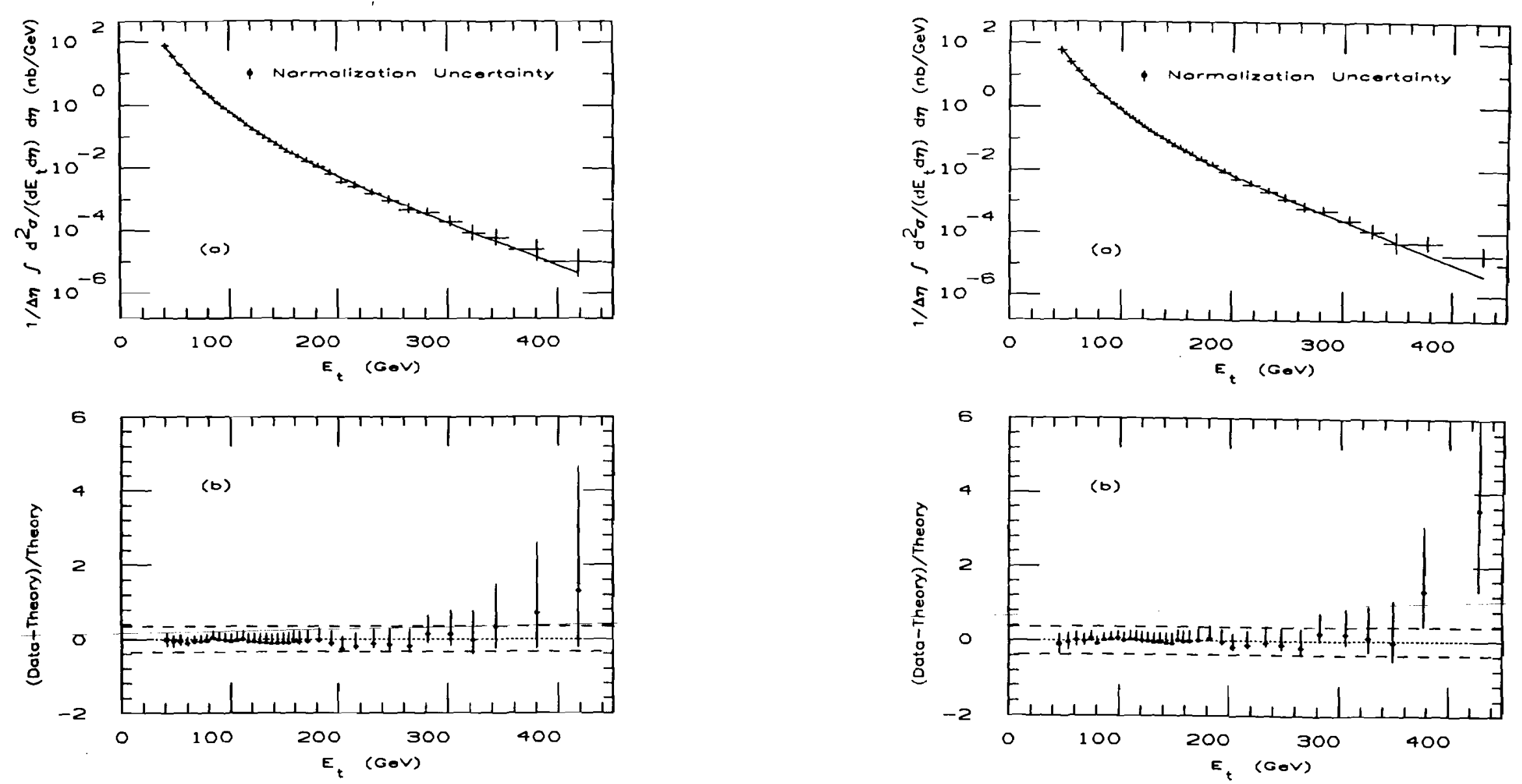

Figure 56. Next-to-leading order QCD calculation of the inclusive jet cross section compare to the measure cross section using the structure function MRS $B$ for a cone size of 0.7. Shown is the a) cross section and b) fractional diflcrence of data with theory.

Figure 57. Next-to-leading order QCD calculation of the inclusive jet cross section compare to the measure cross section using the structure function MRS $B$ for a cone size of 1.0. Shown is the a) cross section and b) fractional difference of data with theory. 

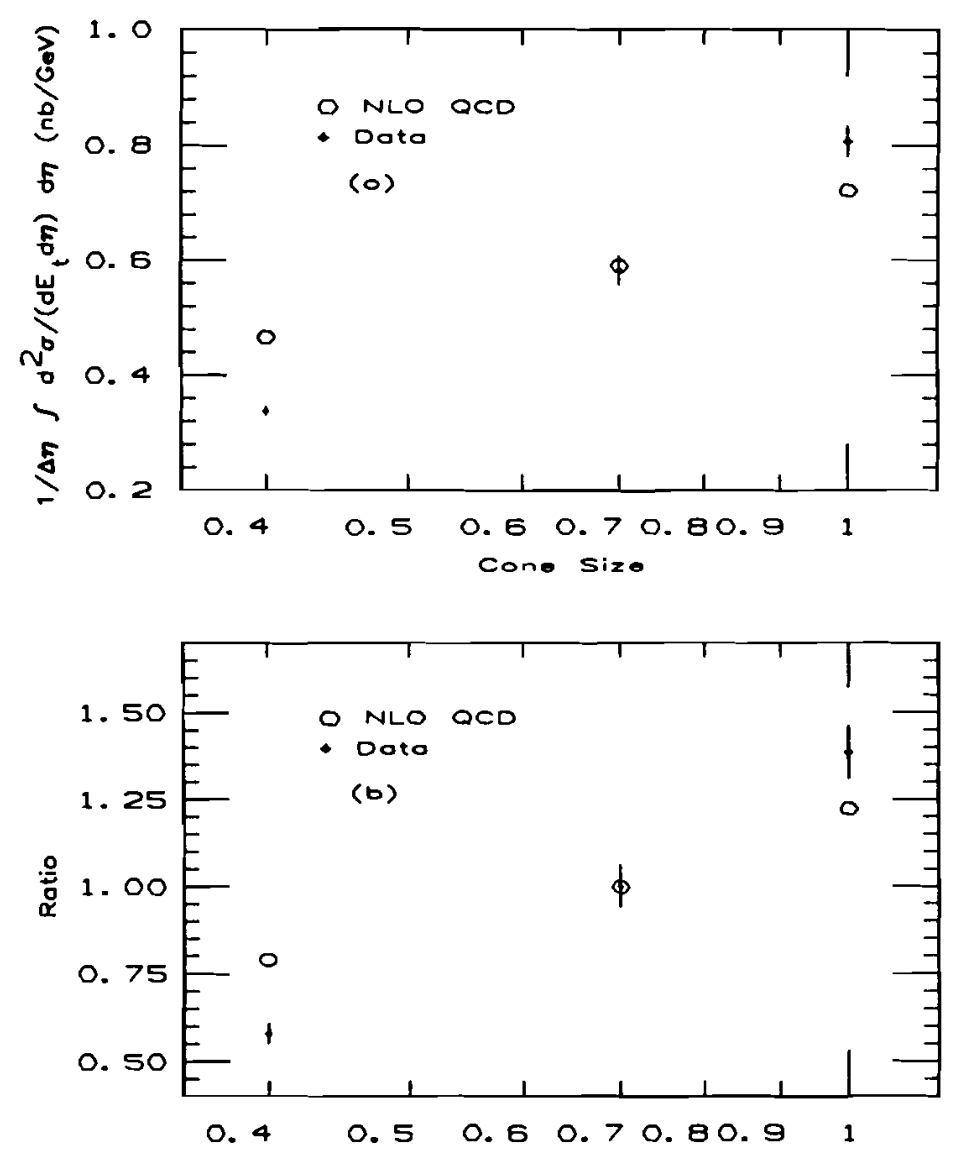

Figure 58. Next-to-leading order QCD calculation of the inclusive jet cross section compare to the measure cross section using the structure function MRS B for $E_{1}=100 \mathrm{GeV}$. Plotted as a function of jet cone size are the (statistical crrors only are plotted) a) cross section and the b) ratio of the cross section for cach cone size to the cross section for a cone size of 0.7 . 


\section{CHAPTER VII}

\section{RESULTS AND CONCLUSIONS}

The inclusive jet cross section has been measured using data collected by the CDF collaboration at $\sqrt{s}=1.8 \mathrm{TeV}$. This measurement was made using three cone sizes of $0.4,0.7$, and 1,0 . The resulting cross section and both statistical and systematic uncertainties are listed in Tables XLII, XLIII, and XLIV for cone sizes of 0.4, 0.7 , and 1.0 respectively. Figures 59,60 , and 61 are plots of the cross scction together with the statistical and $E_{t}$ dependent systematic uncertainties added in quadrature for cone sizes $0.4,0.7$, and 1.0 respectively. The normalization uncertainty plotted in these figures represents the size of the $E_{l}$ independent systematic uncertainty.

The measured cross sections were compared to both leading order and next-toleading order QCD and found to agrec within the listed uncertainties. Although a slight excess of cuents above what is predicted by QCI) was ohserved in the data, this excess was not statistically significant depending on the cloice of structure function used. When comparing to next-to-leading order QCD, the dependence of the cross section a function of cone size was àso measured. T'he data were found to increase in cross section witl larger cone size faster than next-to-leading order QC() predicted.

The cross section was also used to search for quark compositeness, and while no evidence for quark compositeness was found, a lower limit on the value of the compositeness scalc parameter, $\Lambda_{c}$, was set at $1100 \mathrm{GeV}$ at the $95 \%$ confidence level. This can be compared to the previous CDF result of $\Lambda_{c}>700[\mathrm{ieV}$. [26] $A$ comparison can also be made to a linit of $\Lambda_{c}>860 \mathrm{GeV}$ set in muon $\mathrm{g} \cdot 2$ experiments which assurne that quarks and inuons are composed of the same composite particles and that $\Lambda_{c}$ is the same for all quarks and Icptons. [89] The limit set in this dissertation greatly improves the existing limits from CDF and $\mathrm{g}$-2 experiments. It should also Le noted that the CDF ( $p \bar{p})$ limits are less model dependent than the limits set from g. 2 experiments. 
Table XIJI. Continued

\begin{tabular}{|c|c|c|c|}
\hline \multicolumn{4}{|l|}{ Mean } \\
\hline$E_{t}(\mathrm{GeV})$ & Cross Section & \pm Statistical & \pm Systematic (nb/GeV) \\
\hline 36.24 & 86.9 & \pm 1.13 & $\begin{array}{l}+41.9 \\
-32.1\end{array}$ \\
\hline 41.90 & 42.1 & \pm 0.767 & $\begin{array}{l}+20.2 \\
-11.9\end{array}$ \\
\hline 47.52 & 20.9 & \pm 0.539 & $\begin{array}{l}+10.6 \\
-7.52\end{array}$ \\
\hline 53.27 & 11.7 & \pm 0.403 & $\begin{array}{l}+5.88 \\
-4.01\end{array}$ \\
\hline 59.01 & 6.96 & \pm 0.0950 & $\begin{array}{r}+3.43 \\
-2.27\end{array}$ \\
\hline 64.53 & 4.27 & \pm 0.0744 & $\begin{array}{l}+2.12 \\
-1.38\end{array}$ \\
\hline 69.85 & 2.63 & \pm 0.0585 & $\begin{array}{c}+1.38 \\
-0.895\end{array}$ \\
\hline 75.30 & 1.79 & \pm 0.0482 & ${ }_{-0.591}^{+0.913}$ \\
\hline 80.62 & 1.22 & \pm 0.0398 & $\begin{array}{l}+0.624 \\
-0.404\end{array}$ \\
\hline 85.78 & 0.810 & \pm 0.0326 & $\begin{array}{l}+0.439 \\
-0.284\end{array}$ \\
\hline 91.12 & 0.571 & \pm 0.0275 & $\begin{array}{l}+0.312 \\
-0.201\end{array}$ \\
\hline 96.33 & 0.437 & \pm 0.0239 & $\begin{array}{l}+0.226 \\
-0.145\end{array}$ \\
\hline 101.64 & 0.294 & \pm 0.00370 & $\begin{array}{l}+0.165 \\
-0.106\end{array}$ \\
\hline 106.88 & 0.217 & \pm 0.00318 & $\begin{array}{c}+0.123 \\
-0.0782\end{array}$ \\
\hline 112.08 & 0.167 & \pm 0.00279 & ${ }_{-0.0586}^{+0.0925}$ \\
\hline 117.30 & 0.117 & \pm 0.00234 & $\begin{array}{l}+0.0702 \\
-0.0143\end{array}$ \\
\hline 122.74 & 0.0915 & \pm 0.00207 & ${ }_{-0.0335}^{+0.0532}$ \\
\hline 127.94 & 0.0691 & \pm 0.00180 & $\begin{array}{l}+0.0411 \\
-0.0258\end{array}$ \\
\hline 133.12 & 0.0526 & \pm 0.00157 & $\begin{array}{l}+0.0321 \\
-0.0200\end{array}$ \\
\hline 138.30 & 0.0120 & \pm 0.00141 & $\begin{array}{l}+0.0252 \\
-0.0157\end{array}$ \\
\hline 143.46 & 0.0348 & \pm 0.00128 & $\begin{array}{l}+0.0199 \\
-0.0124\end{array}$ \\
\hline 148.83 & 0.0285 & \pm 0.00116 & $\begin{array}{c}+0.0157 \\
-0.00971\end{array}$ \\
\hline 153.88 & 0.0200 & \pm 0.000974 & $\begin{array}{c}+0.0126 \\
-0.00778\end{array}$ \\
\hline
\end{tabular}

\begin{tabular}{|c|c|c|c|}
\hline $\begin{array}{c}\text { Mcan } \\
E_{\ell}(\mathrm{GeV}) \\
\end{array}$ & Cross Section & \pm Statistical & \pm Systematic (nb/GeV) \\
\hline 159.07 & 0.0161 & \pm 0.000875 & $\begin{array}{l}+0.0101 \\
-0.00623\end{array}$ \\
\hline 166.90 & 0.0129 & \pm 0.000555 & $\begin{array}{l}{ }_{-0.00450}^{+0.00734} \\
\end{array}$ \\
\hline 177.03 & 0.00803 & \pm 0.000439 & $\begin{array}{l}+\mathbf{+ 0 . 0 0 4 9 1} \\
-0.00299\end{array}$ \\
\hline 187.49 & 0.00485 & \pm 0.000342 & $\begin{array}{l}+0.00330 \\
-0.00200\end{array}$ \\
\hline 198.23 & 0.00277 & \pm 0.000258 & ${ }_{-0.00134}^{+0.00223}$ \\
\hline 210.38 & 0.00190 & \pm 0.000175 & $\begin{array}{l}+0.00145 \\
{ }_{-0.000870}\end{array}$ \\
\hline 227.23 & 0.00114 & \pm 0.000136 & 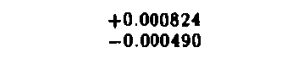 \\
\hline 241.41 & 0.000570 & \pm 0.0000963 & $\begin{array}{l}+0.000523 \\
-0.000309\end{array}$ \\
\hline 259.30 & 0.000456 & \pm 0.0000862 & $\begin{array}{l}+0.000302 \\
-0.000178\end{array}$ \\
\hline 277.46 & 0.000320 & \pm 0.0000628 & $\begin{array}{l}+0.000178 \\
{ }_{-0.000104}\end{array}$ \\
\hline 299.20 & 0.0000985 & $\begin{array}{l}+0.0000487 \\
-0.0000341\end{array}$ & $\begin{array}{l}+0.0000975 \\
-0.0000564\end{array}$ \\
\hline 317.64 & 0.0000737 & $\begin{array}{l}+0.0000412 \\
-0.0000293\end{array}$ & $\begin{array}{l}+0.0000600 \\
-0.0000345\end{array}$ \\
\hline 338.92 & 0.0000368 & $\begin{array}{l}+0.0000359 \\
-0.0000200\end{array}$ & $\begin{array}{l}+0.0000352 \\
-0.0000201\end{array}$ \\
\hline 371.08 & 0.0000247 & $\begin{array}{l}+0.0000241 \\
-0.0000135\end{array}$ & $\begin{array}{c}+0.0000166 \\
-0.00000938\end{array}$ \\
\hline 409.19 & 0.0000111 & $\begin{array}{r}+0.0000147 \\
-0.00000717 \\
\end{array}$ & $\begin{array}{r}+\mathbf{+ 0 . 0 0 0 0 0 7 3 1} \\
-0.00000408 \\
\end{array}$ \\
\hline
\end{tabular}


Table XI.III. Corrected jet cross section for a jet clustering cone size of 0.7 .

\begin{tabular}{|c|c|c|c|}
\hline \multicolumn{4}{|l|}{ Mean } \\
\hline$E_{1}(\mathrm{GeV})$ & Cross Section & \pm Statistical & \pm Systematic $(\mathrm{nb} / \mathrm{GeV})$ \\
\hline 41.63 & 77.4 & \pm 1.08 & $\begin{array}{l}+40.6 \\
-41.9\end{array}$ \\
\hline 47.61 & 37.1 & \pm 0.715 & $\begin{array}{l}+19.9 \\
-19.2\end{array}$ \\
\hline 53.54 & 20.1 & \pm 0.520 & $\begin{array}{l}+10.6 \\
{ }_{-9.47}\end{array}$ \\
\hline 59.93 & 10.5 & \pm 0.373 & $\begin{array}{l}+3.73 \\
-4.72\end{array}$ \\
\hline 66.23 & 6.27 & \pm 0.286 & $\begin{array}{l}+3.30 \\
-2.51\end{array}$ \\
\hline 72.29 & 3.77 & \pm 0.0679 & $\begin{array}{l}+2.03 \\
-1.43\end{array}$ \\
\hline 78.15 & 2.49 & \pm 0.0550 & $\begin{array}{l}+1.32 \\
-0.876\end{array}$ \\
\hline 83.81 & 1.79 & \pm 0.0467 & $\begin{array}{l}+0.893 \\
-0.578\end{array}$ \\
\hline 89.30 & 1.17 & \pm 0.0378 & $\begin{array}{l}+0.624 \\
-0.101\end{array}$ \\
\hline 94.82 & 0.818 & \pm 0.0318 & $\begin{array}{l}+0.442 \\
-0.283\end{array}$ \\
\hline 100.19 & 0.574 & \pm 0.0267 & ${ }_{-0.204}^{+0.321}$ \\
\hline 105.59 & 0.427 & \pm 0.0231 & $\begin{array}{l}+0.236 \\
-0.149\end{array}$ \\
\hline 111.04 & 0.321 & \pm 0.0200 & $\begin{array}{l}+0.175 \\
-0.110\end{array}$ \\
\hline 116.43 & 0.219 & \pm 0.00312 & $\begin{array}{l}+0.131 \\
-0.0819\end{array}$ \\
\hline 121.76 & 0.166 & \pm 0.00272 & $\begin{array}{l}+0.0996 \\
-0.0620\end{array}$ \\
\hline 127.12 & 0.122 & \pm 0.00233 & $\begin{array}{l}+0.0763 \\
-0.0472\end{array}$ \\
\hline 132.49 & 0.0926 & \pm 0.00204 & $\begin{array}{l}+0.0589 \\
+0.0363\end{array}$ \\
\hline 137.77 & 0.0712 & \pm 0.00179 & $\begin{array}{l}+0.0459 \\
-0.0282\end{array}$ \\
\hline 143.05 & 0.0552 & \pm 0.00158 & $\begin{array}{l}+0.0361 \\
-0.0220\end{array}$ \\
\hline 148.48 & 0.0140 & \pm 0.00141 & $\begin{array}{l}+0.0283 \\
-0.0172\end{array}$ \\
\hline 153.71 & 0.0344 & \pm 0.00125 & $\begin{array}{l}+0.0226 \\
-0.0136\end{array}$ \\
\hline 158.92 & 0.0283 & \pm 0.00114 & $\begin{array}{l}+0.0181 \\
-0.0109\end{array}$ \\
\hline 104.24 & 0.0219 & \pm 0.00100 & $\begin{array}{l}+0.0145 \\
-0.00869\end{array}$ \\
\hline
\end{tabular}

'lable XL111. Continued

\begin{tabular}{|c|c|c|c|}
\hline \multicolumn{4}{|l|}{ Mean } \\
\hline$E_{t}(\mathrm{GeV})$ & Cross Section & \pm Statistical & \pm Systematic $(\mathrm{nb} / \mathrm{GeV})$ \\
\hline 171.89 & 0.0165 & \pm 0.000618 & $\begin{array}{l}+0.0106 \\
-0.00634\end{array}$ \\
\hline 182.20 & 0.0111 & \pm 0.000508 & $\begin{array}{l}+0.00711 \\
-0.00421\end{array}$ \\
\hline 193.03 & 0.00656 & \pm 0.000392 & $\begin{array}{l}+0.00473 \\
-0.00278\end{array}$ \\
\hline 203.47 & 0.00368 & \pm 0.000294 & ${ }_{-0.00189}^{+0.00324}$ \\
\hline 215.73 & 0.00256 & \pm 0.000202 & $\begin{array}{l}+0.00211 \\
-0.00122\end{array}$ \\
\hline 231.87 & 0.00157 & \pm 0.000159 & $\begin{array}{l}+0.00123 \\
-0.000703\end{array}$ \\
\hline 246.86 & 0.000903 & \pm 0.000121 & $\begin{array}{l}+0.000759 \\
-0.000431\end{array}$ \\
\hline 264.86 & 0.000470 & \pm 0.0000872 & $\begin{array}{l}+0.000436 \\
-0.000245\end{array}$ \\
\hline 281.96 & 0.000381 & \pm 0.0000685 & $\begin{array}{l}+0.000264 \\
-0.000147\end{array}$ \\
\hline 302.21 & 0.000198 & $\begin{array}{l}+0.0000629 \\
-0.0000490\end{array}$ & $\begin{array}{l}+0.000149 \\
-0.0000821\end{array}$ \\
\hline 322.87 & 0.0000867 & $\begin{array}{l}+0.0000468 \\
-0.0000321\end{array}$ & $\begin{array}{l}+0.0000835 \\
-0.0000467\end{array}$ \\
\hline $\mathbf{3 4 3 . 8 7}$ & 0.0000621 & $\begin{array}{l}+0.0000422 \\
-0.0000269\end{array}$ & $\begin{array}{r}+0.0000498 \\
-0.0000270\end{array}$ \\
\hline 380.71 & 0.0000252 & $\begin{array}{l}+0.0000246 \\
-0.0000137\end{array}$ & $\begin{array}{l}+0.0000205 \\
-0.0000109\end{array}$ \\
\hline 418.54 & 0.0000104 & $\begin{array}{c}+0.0000137 \\
-0.00000671\end{array}$ & $\begin{array}{l}+0.00000881 \\
-0.00000464\end{array}$ \\
\hline
\end{tabular}


Table XIIV. Corrected jet cross section for a jet clustcring cone size of 1.0 .

\begin{tabular}{|c|c|c|c|}
\hline $\begin{array}{c}\text { Mcan } \\
E_{l}(\mathrm{GeV})\end{array}$ & Cross Section & \pm Statistical & \pm Systematic $(n b / G e V)$ \\
\hline 45.78 & 55.8 & \pm 1.14 & $\begin{array}{l}+38.8 \\
-37.0\end{array}$ \\
\hline 53.75 & 25.0 & \pm 0.687 & $\begin{array}{l}+15.9 \\
-14.7\end{array}$ \\
\hline 61.29 & 13.0 & \pm 0.462 & $\begin{array}{l}+7.49 \\
-6.67\end{array}$ \\
\hline 65.10 & 7.09 & \pm 0.323 & $\begin{array}{l}+4.06 \\
-3.45\end{array}$ \\
\hline 71.55 & 4.59 & \pm 0.253 & $\begin{array}{l}+2.39 \\
-1.93\end{array}$ \\
\hline 80.41 & 2.54 & \pm 0.0577 & $\begin{array}{l}+1.53 \\
-1.17\end{array}$ \\
\hline 86.48 & 1.82 & \pm 0.0487 & $\begin{array}{l}+1.00 \\
-0.722\end{array}$ \\
\hline 92.77 & 1.23 & \pm 0.0399 & $\begin{array}{l}+0.675 \\
-0.452\end{array}$ \\
\hline 98.70 & 0.872 & \pm 0.0335 & $\begin{array}{l}+0.473 \\
-0.304\end{array}$ \\
\hline 104.29 & 0.594 & \pm 0.0275 & $\begin{array}{r}+0.343 \\
-0.218\end{array}$ \\
\hline 109.90 & 0.443 & \pm 0.0237 & $\begin{array}{l}+0.252 \\
+0.159\end{array}$ \\
\hline 115.45 & 0.320 & \pm 0.0203 & $\begin{array}{l}+0.187 \\
-0.117\end{array}$ \\
\hline 120.89 & 0.234 & \pm 0.0173 & $\begin{array}{r}+0.142 \\
-0.0879\end{array}$ \\
\hline 126.24 & 0.174 & \pm 0.00281 & $\begin{array}{c}+0.108 \\
-0.0668\end{array}$ \\
\hline 131.64 & 0.128 & \pm 0.00242 & $\begin{array}{l}+0.0835 \\
-0.0511\end{array}$ \\
\hline 137.04 & 0.0995 & \pm 0.00213 & $\begin{array}{l}+0.0648 \\
-0.0393\end{array}$ \\
\hline 142.37 & 0.0747 & \pm 0.00185 & $\begin{array}{l}+0.0507 \\
-0.0306\end{array}$ \\
\hline 147.79 & 0.0576 & \pm 0.00163 & $\begin{array}{l}+0.0398 \\
-0.0239\end{array}$ \\
\hline 153.12 & 0.0496 & \pm 0.00151 & $\begin{array}{l}+0.0316 \\
-0.0188\end{array}$ \\
\hline 158.53 & 0.0373 & \pm 0.00131 & $\begin{array}{l}+0.0251 \\
-0.0148\end{array}$ \\
\hline 163.91 & 0.0299 & \pm 0.00118 & $\begin{array}{l}+0.0201 \\
-0.0118\end{array}$ \\
\hline 171.74 & 0.0218 & \pm 0.000714 & $\begin{array}{l}+0.0146 \\
-0.00851\end{array}$ \\
\hline 182.16 & 0.0149 & \pm 0.000592 & $\begin{array}{l}+0.00974 \\
-0.00560\end{array}$ \\
\hline
\end{tabular}

Table XI.IV. Continued

\begin{tabular}{|c|c|c|c|}
\hline $\begin{array}{c}\text { Mlcan } \\
E_{l}(\mathrm{GeV})\end{array}$ & Cross Section & \pm Statistical & \pm Systematic (nb/GeV) \\
\hline 193.07 & 0.00888 & \pm 0.000458 & $\begin{array}{l}+0.00646 \\
-0.00367\end{array}$ \\
\hline 203.14 & 0.00518 & \pm 0.000350 & $\begin{array}{l}+0.00444 \\
-0.00250\end{array}$ \\
\hline 216.42 & 0.00338 & \pm 0.000232 & $\begin{array}{l}+0.00283 \\
{ }_{-0.00157}\end{array}$ \\
\hline 233.06 & 0.00201 & \pm 0.000179 & $\begin{array}{c}+0.00163 \\
-0.000887\end{array}$ \\
\hline 247.46 & 0.00115 & \pm 0.000136 & $\begin{array}{r}+0.00103 \\
-0.000535\end{array}$ \\
\hline 26.1 .64 & 0.000593 & \pm 0.0000975 & $\begin{array}{l}+0.000612 \\
-0.000325\end{array}$ \\
\hline 282.03 & 0.000496 & \pm 0.0000775 & $\begin{array}{l}+0.000370 \\
{ }_{-0.000394}\end{array}$ \\
\hline 305.43 & 0.000230 & $\begin{array}{l}+0.0000659 \\
-0.0000523\end{array}$ & $\begin{array}{l}+0.000195 \\
-0.000100\end{array}$ \\
\hline 326.53 & 0.000108 & $\begin{array}{r}+0.0000497 \\
-0.0000355\end{array}$ & $\begin{array}{r}+0.000113 \\
-0.0000574\end{array}$ \\
\hline 348.63 & 0.0000479 & $\begin{array}{r}+0.0000381 \\
-0.0000230\end{array}$ & $\begin{array}{l}+0.0000663 \\
{ }_{-0} .0000330\end{array}$ \\
\hline 377.44 & 0.0000480 & $\begin{array}{l}+0.0000288 \\
-0.0000191\end{array}$ & $\begin{array}{l}+0.0000345 \\
-0.0000169\end{array}$ \\
\hline 427.84 & 0.0000193 & $\begin{array}{r}+0.0000153 \\
-0.00000925 \\
\end{array}$ & $\begin{array}{c}+0.0000125 \\
-0.00000593 \\
\end{array}$ \\
\hline
\end{tabular}




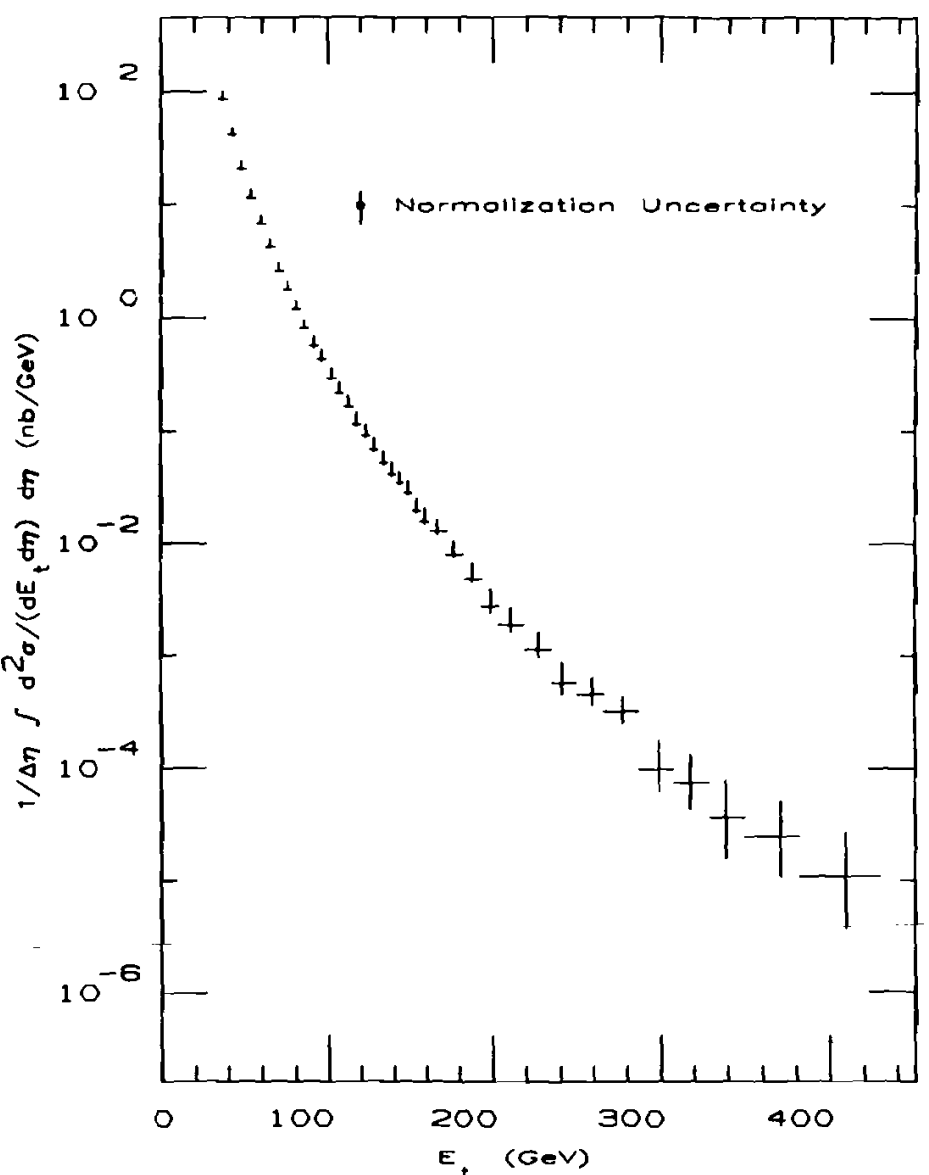

Figure 59. Inclugive jet cross section for a cone size of 0.4 , normalization uncertainty is the $E_{\mathrm{g}}$ independent systematic uncertainty and the error bars represent is the $E_{f}$ independent systematic uncertainty and the error bars represent
the statistical uncertainty added in quadrature with the $E_{t}$ dependent part of the systematic uncertainty.

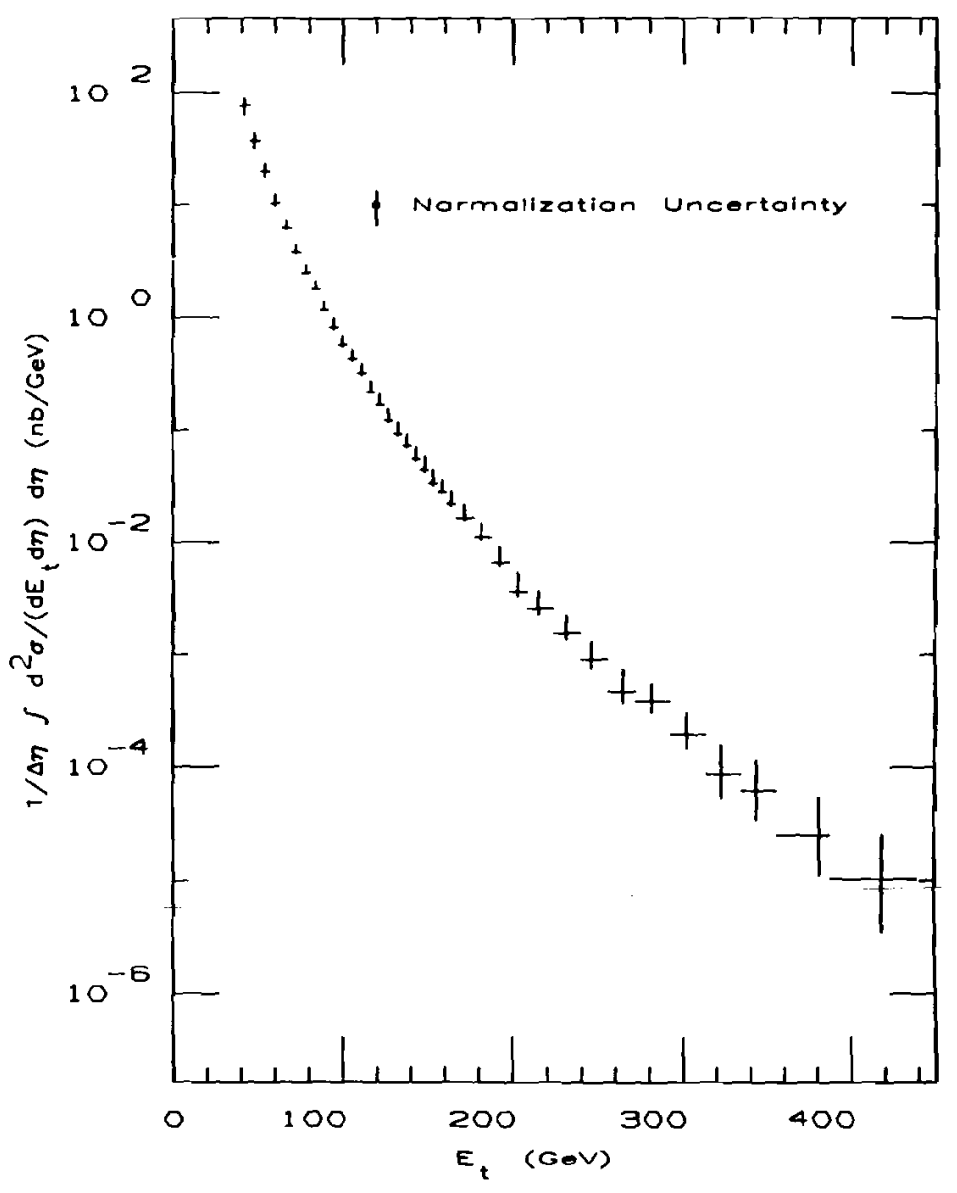

Figure 60. Inclusive jet cross section for a cone size of 0.7 , normalization uncertainty is the $E_{t}$ independent systematic uncertainty and the error bars represent the statistical uncertainty added in quadrature with the $E_{\ell}$ dependent part of the systematic uncertainty. 


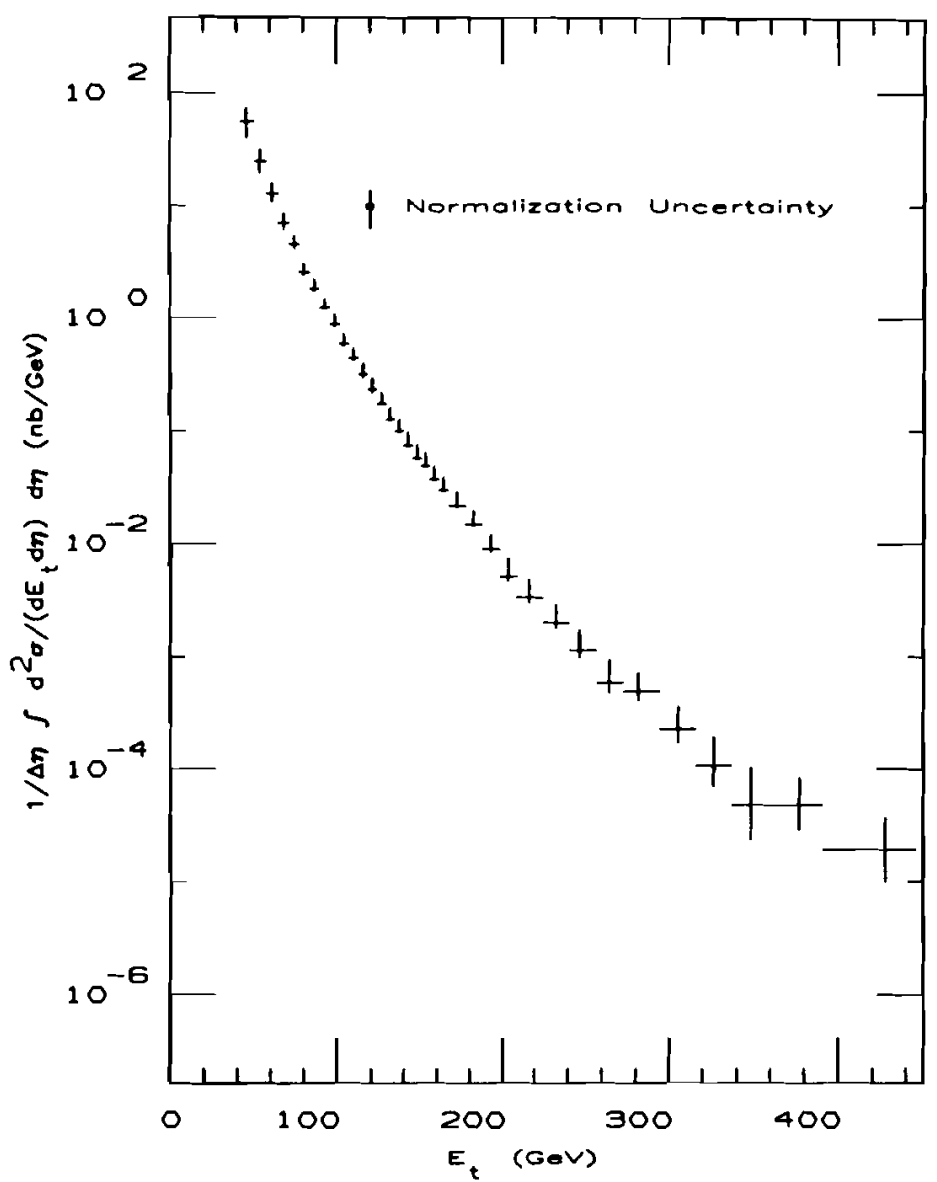

Figure 61. Inclusive jet cross section for a cone size of 1.0, normalization uncertainty is the $E_{t}$ independent systematic uncertainty and the error bars represent the statistical uncertainty added in quadrature with the $E_{t}$ dependent part of the systematic uncertainty. 


\section{REFERENCES}

1 C. Sutton. The Particle Connection. Simon \& Schuster, New York, 1984.

2 F. Close, M. Martin and C. Sutton. The Particle Erplosion. Oxford University Press, New York, 1987

3 Particle Data Group. Review of Particle Properties. Physics Letters, 204B, April 1988

4 V. Barger asid R. Plillips. Collider Physics. Volume7l of Frontiers in Physics, Addison-Wesley Publishing Company, New York, 1087.

5 E. Eicliten, I. Ilinchliffe, K. Lane and C. Quigg. Supercollider Plsysics. Reviews of Modern Physics, 56(4):579-707, October 1984.

6 F. Abe $a t$ al. (CDF Collaboration) Measurement of the Mass and Width of the $Z^{0}$ Boson al the Fermilab Tevatron. Physical Review Letters, 63(7):720 723, August 1989.

7 G. S. Abrams el al. (Mark II Collaboration). Initial Measurements of Z-Boson Resonance Parameters in $\mathrm{e}^{+} \mathrm{e}^{-}$Annihilation, Physical Review Letters, 63(7):724-727, August 1989

8 B. Adeva et al. (L3 Collaboration). Determination of the Properties of the Neutral lintermediate Vector Boson $Z^{0}$. Physics Letters, 231B:509, 1989.

9 D. Decamp et al. (ALEPII Collaboration). Determination of the Number of Light Neutrino Species. Physics Letters, 231B:519, 1989.

$10 \mathrm{M}$. Akrawy ct al. (OPAL Collaboration). Measurement of the $Z^{0}$ Mass and Width with the OPAL Detector at LEP. Plysics letters, 231B:530, 1989.

11 P. Aarnio et al (DELPIIl Collaboration). The Mass and Width of the $Z^{0}$-Particle from Multihadronic Final States Produced in $e^{+} e^{-}$Annihilations. Plyysirs Letters, 231B:539, 1989.

12 F. Abe et al. (CDF Collaboration). A Search for the Top Quark in the Reaction $\bar{p} p \rightarrow$ Electron + Jets at $\sqrt{s}=1.8 \mathrm{TeV}$. Physical Revicw Letters, 64(2): 142, January 1990

13 F. Abe et al. (CDF Collaboration). Search for New lleavy Quarks in Electron-Muon Events at the Fermilab Tevatron Collider. Physical Review Letters, 64(2):147 January 1990 .
14 F. Abe et al (CDF Collaboration). Search for the Top Quark at CDF. To be published in the proceedings of the PANIC XII, International Conference on Particics and Nuclei, Massaclusetts Institute of Technology, Cambridge, Massarlusetts, June 1990

15 I. Aitchison and A. lley. Gauge Theories in Particle Physics. Graduate Student Scrics in Physics, Adarn llilger, Pliladelphia, 2nd edition, 1989.

16 D). Perkins. Introduction to /ligh Energy Physics. Addison-Wesley P'ublishing ( ompany Inc., Reading Massarhusette, 3rd colition, 1987.

17 F. Ilatzen and A. Martin. Quarks \& Leptons: An Introduclory Course in Modern Particle Physics. Jolın Wiley \& Sons, New York, 1984.

18 T. Bowcock et al. (CI.EO Collaboration). Search for Fractionally Charged Particles in $c^{+} e^{-}$Annililations at $\sqrt{s}=10.5$ GeV. Physical Review, D40:263, 1989.

19 E. Eichten, K. Lane and M. Peskin. New Tests for Quark and Lepton Substructure. Plyysical Review Letters, 50(11):811 -814, March 1983.

20 S. Ellis, Z. Kunszt and D. Soper. One-Jet Inclusive Cross Section at Order $\alpha^{3}$ : Gluons Only. Plysical Review Letters, 62(7):726-729, February 1989.

21 S. Ellis, Z. Kunszt and D. Soper. One-Jet Inclusive Cross Section at Order $\alpha_{\&}^{3}$ : Quarks and Gluons. Plyysical Review Letters, 64(18):2121, April 1990.

22 F. Aversa, M. Greco, P. Chiappetta and J. Ph. Guillet. LNF-90/012 PT (1990), Submitted to Pliysical Review Letters.

23 T. Akrsson at at. (Axial Field Spectrometer Collaboration). The Jet Cross Scction in pp luteractions at $\sqrt{8}=45 \mathrm{GeV}$ and It's $\sqrt{9}$ Dependence. Physical l.etters, 123B(1,2):133-138, March 1983

24 G. Arnison et al. (UA1 collaboration). Measurement of the Inclusive Jet Cross Section at the CERN $p \bar{p}$ Collider. Physical Letters, 172B(3,4):461, 1986.

25 P. Bagnaia el al. (UA2 Collaboration). Measurement of Very Large Transverse Momenturn Jet Production at the CERN $\bar{p}$ p Collider. Physical Letters, $138 B(5,6): 430-440$, April 1984

26 F. Abe et al. (CDF collaboration). Measurement of the Inclusive Jet Cross Section in $\vec{p}$ Collisions at $\sqrt{\mathbf{s}}=1.8 \mathrm{TeV}$. Plyysical Review Letters, 62(6):613-616, February 1989. 
27 E. Eicluten, I. Ilinchliffe, K. Lane and (. Quigg. Superrollicler Physics. Reviews of Modern Plyysics, 56(4):579, October 198.1.

28 R. Ellis and J. Sexton. QCD Radiative Corrections to Parton-Parton Scattering. Nuclear Physics, B269:445-484, 1986.

29 F. Abe et al. (CDF collaboration). The CDF Detector: An Overview. Nuclear Instruments and Methods in Physics Research, A271:387-403, 1988.

30 F. Snider $a t$ al. The CDF Vertex Time Projection Chamber System. Nuclear Instruments and Methods in Plysics Research, A208:75-.91, 1988.

31 F. Bedeschi et al.. Design and Construction of the CUF Central 'Tracking Cliamber. Nuclear Instruments and Metliods in Pliysics Research, A268:50-74, 1988

32 S. Bliadra et al. The Design and Construction of the CDF Central Drift Tube Array. Nuclear Instruments and Methods in l'hysics Research, A 268:92-104, 1988 .

33 M. Atac el al.. Radial Wire Drift Chambers for the CDF Forward Tracking Nuclear Instruments and Methods in Physics Research, A 269:40-50, 1988.

34 L. Balka et al.. The CDF Central Electromagnetic Calorimeter. Nuclear Instruments and Methods in Physics Research, A267:272-279, 1988.

35 S. Bertolucci et al. The CDF Central and Endwall IJadron Calorimeter. Nuclear Instruments and Methods in Physics Research, A 267:301-314, 1988.

36 Y. Fukıi et al.. CDF End Plug Electromagnetic Calorimeter Using Conductive Plastic Proportional Tubes. Nuclear Instruments and Methods in Physics Research, A 267:280-300, 1988

37 W. C. Carithers et al. Performance and Calibration Studics of the CDF Endplug Calorimeter. In the proceedings of the Gas Sampling Calorimeter Workshop II, Fermilab, 1985

38 G. Bradenburg et al.. An Electromagnetic Calorimeter for the Small Angle Regions of the Collider Detector at Fermilab. Nuclear Instruments and Methods in Physics Research, A267:257-271, 1988.

39 S. Cilangir et al.. The CDF Porward/Backward Hladron Calorimeter. Nuclear Instruments and Methods in Plysics Research, A267:219 256, 1988.
10 G. Ascoli ef al. CDF Central Muon Detector. Nuclear lustruments and Methods in Physics Research, A268:33-40, 1988.

11 K. Byrum ef al.. The CIF Forward Muon System. Nuclear Instruments and Mcthods in l'hysics lesearch, A268:46-49, 1988.

2 G. Drake et al CDF liront End Electronics: The RAHBIT System. Nuclear Instrunients and Methods in Physics Research, A 269:68-81, 1988.

43 E. Barsotti et al.. FAS'TBUS Data Acquisition for CDF. Nuclear Instruments and Methods in Physics Research, A269:82-92, 1988.

44 D. Amilei $t$ al. A Two Level FASTBUS Based Trigger System for CDF. Nuclear Instruments and Methods in Physics Research, A 269:51-62, 1988.

45 D. Amidei at al. A Two Level FAS'TBUS llased 'Trigger System for CDF. Nuclear lnstruments and Methods in Physics Research, A269:51-62, 1988.

46 J. A. Appel et al. (UA2 Collaboration). Measurement of the $\sqrt{s}$ Dependence of Jet Production at the CERN ppp Collider. Physical Letters, 180B:349, October 1990.

17 D. Brown et al. Clustering Algorithms and Their Performance. CDF Note 605 , Collider Detector at Fermilab, Batavia, Illinois, February 1988.

48 J. llauger Guide to the Triggers Used in the 1988-1989 CDF Data Run CDF Note 966, Collider Detector at Fermilab, Batavia, Illinois, June 1989.

49 T. Carroll et al. The CDF LEVEL3 Trigger. Talk presented at Workshop on Triggering and Data Acquisition for Experiments at the Superconducting Super Collider in Toronto Canada. CDF Note 895, Collider Detector at Permilab, Batavia, Illinois, January 1989

50 S. Cihangir et al Neuiron Induced Pulses in CDF Forward Hadron Calorimeter. Submitted to IEEE Symposium in Orlando, Florida November 911,1988 . CDF Note 810, Collider Detector at Fermilab, Batavia, Illinois November 1988

51 W 'Trischuk and I Nodulunan. 1988-89 Missing E Ceulering and Resolution. CDF Note 948, Collider Detector at Fermilab, Batavia, Illinois, June 1989.

52 W. Trischuk, L. Nodulman and P. Hu. The Tail of the MET Spin (I)F Note 962, Collider Detector at Fermilab, Batavia, Illinoig, June 1989. 
53 D. Brown and M. Franklin. FILT GAS CDF Note 811, Collider Detector at Fermilab, Batavia, Illinois, November 1988.

54 P. Ilurst and M. Franklin. Cable Noise Filler Description: PIIA. CDF Note 847, Collider Detertor at Fermilab, Batavia, Illinois, December 1988.

55 P. Ilurst, M. Franklin and T. Kamon. Cable Noise Filter Description: FIII. CIJF Note 848, Collider Detector at Fermilab, Batavia, Illinois, December 1988.

56 T. Kamon, P. Ilurst, M. Franklin and Y. Funayama. Description of FIIA NCABLE. CDF Note 900, Collider Detector at Fermilab, Batavia, Illinois, March 1989

57 G. Redlinger and Y. D. Tsai. The Iladron TDC Filler for Mfain Ring and Cosmic Ray Events. CDF Note 521, Collider Detcctor at Fermilab, Batavia, Illinois, June 1987.

58 S. Kuhlmann and J. Yoh. Fillering Jet Backgrounds in the CDF' Central Detector. CDF Note 548, Collider Detector at Jermilab, Batavia, Illinois, August 1987.

59 T. Ilessing. Filtering Central Jet Backgrounds in the 1988-1989 Data. CDF Note 957, Collider Detector at Fermilab, Batavia, llinois, June 1989.

60 T. Kamon. Cosmic Ray Muon Background to Missing El Physics at the CDF - Rate Estimate for 1988-89 Data -. CDF Note 979, Collider Detector at Fermilab, Batavia, Illinois, July 1989.

61 S. Kullimann. Acceptance Issues for the Inclusive Jet Cross Scction. CDF Note 711, Collider Detector at Fermilab, Batavia, Illinois, July 1988.

62 S. Kulilmann. Inclusive Central Jet Production at $\sqrt{s}=1.8 \mathrm{TrV}$. PH. I). Thesis, Purdue University, August 1988.

63 S. Kuhlmann. Steps in Deriving the Inclusive Jet Cross Section. CDF Note 709, Collider Detector at Fermilab, Batavia, Illinois, July 1988.

64 J. Iluth and S. Behrends. Statistical Issurs for the Inclusive Jel E $E_{t}$ Paper. CDF Note 747, Collider Detector at Fermilab, Batavia, Illinois, September 1988.

65 S. Behrends, M. D. Slıapiro, and E. Pare. Single Pion Response in the Central Calorimeter. CDF Note 1066, Collider Detector at Fermilab, Batavia, Illinois, November 1989
66 D. Brown, S. Liannappan, and M. D. Shapiro. Single Pion Response in the (entral Calorimeter. CDF Note 753, Collider Detector at Fermilab, Batavia, Hlinois, October 1988.

67 T' Ilessing, S. Behrends, P. Tipton, and B. Winer. Nonte Carlo Fragmentation Tuning for Jet Encrgy and Resolution Analysis. CDF Note 1131, Collider Betector at Fermilab, Batavia, Illinois, February 1990.

68 II. P. Feymman and R. D. Field. A Parameterization of the P'roperties of Quark Juts. ivucicar P'ilysics, B136:1, 1978.

69 1). Brown and S. Kannappan. SETIRT, a Single Parton Fragmentation Module. (IDF Note 873, Collider Detcetor at Fermilab, Batavia, Illinois, February 1989 .

70 'T. Ilessiug and S. Belirends. Inclusive Jet Et Spectrum: Energy Corrections and Resolution Unsmearing. CDF Note 1132, Collider Detector at Fermilab, Batavia, Illinois, March 1990.

71 R. St. Deus. Implications for the Top in Low Energy Jets from Gamma-Jet Fvents. (DF Note 891, Collider Detector at Fermilab, Batavia, Illinois, March 1989.

72 S. Kuhlmann et al.. Central Jet Energy/Momentum Corrections. CDF Note 686, Collider Detector at Fermilab, Batavia, Illinois, July 1988.

73 P. Bagnaia et al. (UA2 collaboration). Measurement of Jet Production Properties at the CERN $p \bar{p}$ Collider. Physical Letters, 144B:283, 1984.

74 P. Bagnaia et al. (UN2 collaboration). Measurement of Production and Properties of Jets at the CEIRN $p \bar{p}$ Collider. Zeitschrift für Physik, C20:117, 1983.

75 R. St. Denis. Dijet Angular Distributions in Proton-Antiproton Collisions at the Fermilab Tevatron. Ph. D. Thesis, Ilarvard University, December 1988.

76 R. Ilarris. Two Jet Differential Cross Section and Structure Functions in $\bar{p} p$ Collisions at $\sqrt{s}=1.8 \mathrm{TeV}$. Ph.D. Thesis, University of California at Berkeley, August 1989

77 R. M. Ilarris. Unsmearing the Single Jet Inclusive Distribution. CDF Note 606 ('ollider Detector at Fermilab, Batavia, Illinois, June 1988. 
78 S. Behrends, and S. Kullnaann. Checks of the CDFSIM/ Central Calorimeter Simulation. CDF Note 684, Collider Detector at Fernilab, Batavia, Illinois, May 1988.

79 B. Flaugher and S. Kuhlinann. Underlying Event Energy in Clusters. CDF Note 685, Collider Detector at Fermilab, Batavia, Illinois, May 1988

80 R. Alssourge et al. (UA5 Collaboration). Difraction Dissociation at the CERN Pulsed pī Collider at c.m. Energies of 900 and 200 GeV. Zeitschrift fïr Physik, C33:175, 1986.

$8 \mathrm{I}$ G. Alner et al. (UA5 Collaboration). Antiproton-l'roton Cross Sections at 200 and $900 \mathrm{GeV}$ c.m. Energy. Zeitschrifl für Physik, C32:153, 1986.

82 M. Bozzo et al. (UA4 Collaboration). Measurements of the Proton-Antiproton Total and Elastic Cross Sections at the CERN SPS Collider. Physics Letters, 147B:392, 1984.

83 Carla Grosso-Pilcher and S. White. CDF Luminosity Calibration. CDF Note 1202, Collider Detector at Fermilab, Batavia, Illinois, A ugust 1990.

84 Data exists from several deep inelastic clectron, muon, and neut rino experiment the list includes: SI,AC-MIT Collaboration electron scattering measurements, European Muon Collaboration (EMC) muon measurements, Big European Bubble Chamber (BEBC) Collaboration, CERN-Dortmund-Ileidelberg-Sacaly (CDIIA) Collaboration and, CIIARM Collaboration neutrino measurements, as well as data from the BCDMS Collaboration, the CCFRR Collaboration.

85 D. Duke and J. Owens. $Q^{2}$-dependent Parameterizations of Parton Distribution Functions. P'hysical Review, D30(1):49, July 1984.

86 M. Diemoz, F. Ferroni, E. Longo and G. Martinelli. Parton Densities from Deep Inclastic Scattering to lladronic Process at Super Collider Energies. Zeitschrift für Physik, C39:21-37, 1988.

87 A. Martin, R. Roberts, and W. Stirling. Structure-function Analysis and ", Jet, W, and Z Production: Determining the Gluon Distribution. Physical Review, D37:1101, 1988

88 W. Tung, et al.. Fermilab P'reprint. Fermilab-Pub-90/24, 1990

89 M. Abolins et al.. Testing the Compositeness of Quarks and Leptons. In thic procecdings of the 1982 DP F Summer Study on Elementary Particle P'hysics and Future Facilities, 1982 


\section{APPENDIX A}

\section{DIJET BALANCING}

Dijet balancing is a technique first introduced by the UA2 collaboration|73, 74] and has been used extensively in jet measurements at CDF.[75, 76] A dijet event is defined by the olsservation in the deteitur uf two jets which origmate from the scaltering partons in the $\bar{p} \bar{p}$ collision. Dijet $\vec{k}_{t}$ is defined as the vector sum of the transverse monentum of these jets. In the absence of initial state radiation, conservation of momentum reguires the total transverse monentum $\left(\boldsymbol{\rho}_{T}\right)$ of the scattered jets int the event to be conserved. In dijet events, this implies that $\vec{k}_{t}$ is expected to be zero. Detector resolution and QCD bremsstralılung produce a momentum inbalance in the event and the $\vec{k}_{1}$ is not zero.

\section{A. Dijet $\vec{k}$}

The coordinate system for dijet $\vec{k}_{t}$ is defined so that the perpendicular $(\hat{i})$ direction is the direction that bisects the azimuthal angle between the two jets, and the parallel (i) direction is the orthogonal direction such that $\hat{\|} \times \hat{i}=\hat{z}$, where $\hat{z}$ is defined to be the positive z-axis in the detector coordinate systenn. Figure 62 shows all exaluple of $\vec{k}_{8}$ for a two jet event.

Defining the coordinate system in this ways inplies,

$$
\begin{aligned}
& k_{t_{1}}=\left(P_{t_{1}}+P_{t_{2}}\right) \cos \frac{\phi_{12}}{2}, \\
& k_{t_{11}}=\left(P_{t_{1}}-P_{t_{2}}\right) \sin \frac{\phi_{12}}{2}
\end{aligned}
$$

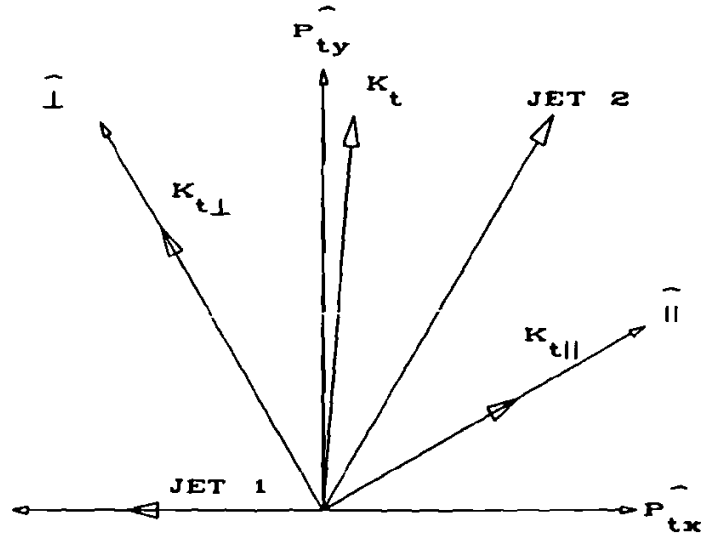

Figure 62 . Illustration of dijet $\overrightarrow{k_{t}}$ coordinate system.

where $\phi_{12}$ is the azimnthal angle separating the two jets and $P_{t_{i}}$ is the magnitude of the $P_{t}$ for jet $i(i=1$ or 2$)$. $E_{t}$ is related to $P_{t}$ by

$$
E_{t}^{2}=p_{i}^{2}+m^{2}
$$

where in is the inass of the jet. The sum or difference in $E_{l}$ for two jets therefore is

$$
\begin{aligned}
& E_{t_{1}} \pm E_{t_{2}}=\sqrt{P_{t_{1}}^{2}+m_{1}^{2}} \pm \sqrt{P_{t_{2}}^{2}+m_{2}^{2}} \\
& =P_{i_{1}} \sqrt{1+\frac{m_{1}^{2}}{P_{t_{1}}^{2}}} \pm P_{t_{2}} \sqrt{1+\frac{m_{2}^{2}}{P_{t_{2}}^{2}}}, \\
& \simeq P_{t_{1}}\left(1+\frac{m_{1}^{2}}{2 P_{t_{1}}^{2}}\right) \pm P_{t_{2}}\left(1+\frac{m_{2}^{2}}{2 P_{t_{2}}^{2}}\right)+O\left(\frac{m^{4}}{P_{1}^{4}}\right), \\
& =P_{t_{1}} \pm P_{t_{2}}+\left(\frac{m_{1}^{2}}{2 P_{t_{1}}^{2}} \pm \frac{m_{2}^{2}}{2 P_{t_{2}}^{2}}\right)+O\left(\frac{m^{4}}{P_{t}^{4}}\right) \text {. }
\end{aligned}
$$


The ratio of jet mass to jet $P_{t}$ is small,[75] therefore from Equation 5 ,

$$
E_{t_{1}} \pm E_{t_{2}} \simeq P_{t_{1}} \pm P_{t_{1}}
$$

Ilence,

$$
k_{t_{1}}=\left(E_{t_{1}}+E_{t_{2}}\right) \cos \frac{\phi_{12}}{2}
$$

and

$$
k_{t_{\|}}=\left(E_{t_{1}}-E_{t_{2}}\right) \sin \frac{\phi_{12}}{2}
$$

Angular deviations in the measurements of these two jets can be observed in the perpendicular component of dijet $\vec{k}_{t}\left(k_{l_{1}}\right)$. $E_{t}$ imbalance in the dijet system is observed in the measurement of the parallel component of dijet $\vec{k}_{t}\left(k_{t_{1}}\right)$.

\section{Jet Resolution}

The jet energy resolution in the calorimeter can be parameterized [3] as,

$$
\frac{\sigma(E)}{E}=\frac{A}{\sqrt{(E)}}
$$

$E_{l}=E \sin \theta$, where $\theta$ is the angle of the jet with respect to the proton beam direction ( $z$ or the polar angle). In the central region of the CDF detector $\sin \theta \sim 1$, therefore $E_{t} \simeq E$ and the jet $E_{t}$ resolution can be written as

$$
\frac{\sigma\left(E_{t}\right)}{E_{t}}=\frac{A}{\sqrt{E_{t}}}
$$

or

$$
\sigma\left(E_{t}\right)=A \sqrt{E_{1}}
$$

Assuming independent errors on the resolution for the two jets, then

$$
\sigma\left(E_{t_{1}}-E_{i_{2}}\right)=\sqrt{\sigma^{2}\left(E_{1_{1}}\right)+\sigma^{2}\left(E_{i_{2}}\right)} .
$$

The value of $A$ in Equation 57 should be the same for both jets if they are in the central calorimeter and $E_{1_{1}} \sim E_{t_{2}} \equiv E_{l}$, therefore

$$
\begin{aligned}
& \sigma\left(E_{t_{1}}-E_{t_{2}}\right)=A \sqrt{2 E_{t}}, \\
& \frac{\sigma\left(E_{t_{1}}-E_{t_{2}}\right)}{\sqrt{2}}=\sigma\left(E_{t}\right) .
\end{aligned}
$$

The effects of QCD radiation and angular mismcasurement are present in both $k_{t_{\perp}}$ and $k_{t_{\mid l}}$; however, as can be seen in Figure 63, the distribution of $k_{t_{\perp}}$ is flat (constant in $E_{t}$ ) whilc there is an $E_{t}$ dependence to $k_{t \mid l}$ due to the jet $E_{t}$ resolution. Thereforc, by subtracting $\sigma\left(k_{t_{1}}\right)$ in quadrature from $\sigma\left(k_{l_{1 \mid}}\right)$, the jet $E_{t}$ resolution can be isolated from these effects.[75] Defining,

$$
\sigma^{\prime}\left(k_{l}\right)=\sqrt{\sigma^{2}\left(k_{l \mid l}\right)-\sigma^{2}\left(k_{l_{1}}\right)}
$$

and since $\sigma\left(k_{t_{1}}\right)$ is equivalent to $\sigma\left(E_{t_{1}}-E_{t_{2}}\right)$, then

$$
\sigma\left(E_{t_{1}}-E_{t_{2}}\right) \approx \sigma^{\prime}\left(k_{t_{1}}\right)
$$

$$
\sigma\left(E_{\ell}\right) \approx \frac{\sigma^{\prime}\left(k_{\ell}\right)}{\sqrt{2}}
$$

Thus, by using dijet $\vec{k}_{\mathfrak{t}}$ balancing techniques, single jet $E_{t}$ resolution can be extracted from the data. 


\section{APPENDIX B}

\section{THE CDF COLLABORATION}

F. Abe, ${ }^{(8)}$ D. Amidei, ${ }^{(1)}$ G. Apollinari, ${ }^{(11)}$ M. Atac, ${ }^{(1)}$ P. Auchincloss, ${ }^{(14)}$ A. R. Baden, ${ }^{(6)}$ A. Bamberger, ${ }^{(19)}$ A. Barbaro-Galtieri, ${ }^{(9)}$ V. E. Barnes, ${ }^{(12)}$ F. Bedeschi, ${ }^{(11)}$ S. Behrends, ${ }^{(2)}$ S. Belforte, ${ }^{(11)}$ G. Bellettini, ${ }^{(11)}$ J. Bellinger, ${ }^{(18)}$ J. Bensinger, ${ }^{(2)}$ A. Beretvas, ${ }^{(4)}$ J. P. Berge, ${ }^{(1)}$ S. Bertolucci, ${ }^{(5)}$ S. Bhadra, ${ }^{(7)}$ M. Binkley, (1) R. Blair, ${ }^{(1)}$ C. Blocker, ${ }^{(2)}$ A. W. Booth, ${ }^{(1)}$ T. Bowcock, ${ }^{(15)}$ G. Brandenburg, ${ }^{(6)}$ D. Brown, ${ }^{(6)}$ E. Buckley, ${ }^{(14)}$ A. Byon, ${ }^{(12)}$ K. L. Byrum, ${ }^{(18)}$ C. Campagnari, ${ }^{(3)}$ M. Campbell, ${ }^{(3)}$ R. Carey, ${ }^{(6)}$ W. Carithers, ${ }^{(9)}$ D. Carlsmith, ${ }^{(18)}$ J. T. Carroll, (4) R. Cashmore, ${ }^{(19)}$ F. Cervelli, ${ }^{(11)}$ K. Chadwick, ${ }^{(1)}$ G. Chiarelli, ${ }^{(5)}$ W. Chinowsky, ${ }^{(9)}$ S. Cihangir, ${ }^{(1)}$ A. G. Clark, ${ }^{(1)}$ D. Connor, ${ }^{(10)}$ M. Contreras, ${ }^{(2)}$ J. Cooper, ${ }^{(1)}$ M. Cordelli, ${ }^{(5)}$ D. Crane, ${ }^{(1)}$ M. Curatolo, ${ }^{(5)}$ C. Day, ${ }^{(1)}$ S. Dell'Agnello, ${ }^{(1)}$ M. Dell'Orso, ${ }^{(1)}$ L. Demortier, ${ }^{(2)}$ P. F. Derwent, ${ }^{(3)}$ T. Devlin, ${ }^{(14)}$ D. DiBitonto, ${ }^{(15)}$

R. B. Drucker, ${ }^{(9)}$ J. E. Elias, ${ }^{(1)}$ R. Ely, ${ }^{(9)}$ S. Errede, ${ }^{(7)}$ B. Esposito, ${ }^{(5)}$ B. Flaugher, ${ }^{(14)}$ G. W. Foster, ${ }^{(4)}$ M. Franklin, ${ }^{(6)}$ J. Freeman, ${ }^{(1)}$ II. Frisel, ${ }^{(3)}$ Y. Fukui, ${ }^{(8)}$ Y. Funayama, ${ }^{(16)}$ A. F. Garfinkel, ${ }^{(12)}$ A. Gauthier, ${ }^{(7)}$ S. Gecr, ${ }^{(6)}$ P. Giannetti, ${ }^{(11)}$ N. Giokaris, ${ }^{(13)}$ P. Giromini, ${ }^{(5)}$ L. Gladncy, ${ }^{(10)}$ M. Gold, ${ }^{(9)}$

K. Goulianos, ${ }^{(13)}$ II. Grassmann, ${ }^{(11)}$ C. Grosso-Pilcher, ${ }^{(3)}$ C. Ilaber, ${ }^{(9)}$ S. R. IIalın, ${ }^{(1)}$ R. Ilandler, ${ }^{(18)}$ K. Ilara, ${ }^{(16)}$ R. M. Harris, ${ }^{(9)}$ J. Ilauser, $^{(3)}$ C. Ilawk, ${ }^{(14)}$ T. Ilessing, ${ }^{(15)}$ R. Ilollebeek, ${ }^{(10)}$ L. Ilolloway, ${ }^{(7)}$ P. IIu, ${ }^{(11)}$ B. Ilubbard, ${ }^{(9)}$ B. T. Iluff man, ${ }^{(12)}$ R. Ilughes, ${ }^{(10)}$ P. Ilurst, ${ }^{(7)}$ J. Iluth, ${ }^{(1)}$ M. Incagli, ${ }^{(11)}$ T. Ino, ${ }^{(16)}$ II. Iso, ${ }^{(16)}$ II. Jensen, ${ }^{(1)}$ C. P. Jessop, ${ }^{(6)}$ R. P. Jolınson, ${ }^{(1)}$ U. Joshi, ${ }^{(1)}$ R. W. Kadel, ${ }^{(1)}$
T. Kamon, ${ }^{(15)}$ S. Kanda, ${ }^{(16)}$ D. A. Kardelis, ${ }^{(7)}$ I. Karliner, ${ }^{(7)}$ E. Kearns, ${ }^{(6)}$ 1R. Kin, ${ }_{1.11},{ }^{(1)}$ P. Kesten, ${ }^{(2)}$ R. M. Keup, ${ }^{(7)}$ If. Keutelian, ${ }^{(7)}$ S. Kim, ${ }^{(16)}$ L. Kirsch, ${ }^{(2)}$ K. Kondo, ${ }^{(16)}$ S. E. KulıImann, ${ }^{(1)}$ E. Kuns, ${ }^{(14)}$ A. T. Lasanen, ${ }^{(12)}$ J. 1. Lamoureux, ${ }^{(18)}$ W. Li, ${ }^{(1)}$ T. M. Liss, ${ }^{(7)}$ N. Lockyer, ${ }^{(10)}$ C. B. Luchini, ${ }^{(7)}$ P. Maas, ${ }^{(1)}$ M. Mangano(11) J. P. Marriner, ${ }^{(1)}$ R. Markelof, ${ }^{(18)}$ L. A. Markosky, ${ }^{(18)}$ R. Mattingly, ${ }^{(2)}$ P. Mclntyre, ${ }^{(15)}$ A. Menzione, ${ }^{(1)}$ T. Meyer, ${ }^{(15)}$ S. Mikamo, ${ }^{(8)}$ M. Miller, ${ }^{(3)}$ 'T. Mimashi, ${ }^{(16)}$ S. Miscetti, ${ }^{(5)}$ M. Mishina, ${ }^{(8)}$ S. Miyashita, ${ }^{(16)}$ Y. Morita, ${ }^{(16)}$ S. Moulding, ${ }^{(2)}$ A. Mukherjee, ${ }^{(1)}$ L. F. Nakae, ${ }^{(2)}$ I. Nakano, ${ }^{(16)}$ C. Nelson, ${ }^{(1)}$ C. Newinan-Ilolmes, ${ }^{(1)}$ J. S. T. Ng, ${ }^{(6)}$ M. Ninomiya, ${ }^{(6)}$ L. Nodulınan, ${ }^{(1)}$ S. Ogawa, ${ }^{(16)}$ R. Paoletti, (11) A. Para, ${ }^{(1)}$ E. Pare, ${ }^{(6)}$ J. Patrick, ${ }^{(1)}$ T. J. Phillips, ${ }^{(6)}$ R. Plunkett, ${ }^{(1)}$ L. Pondrom, ${ }^{(18)}$ J. Proudfoot, ${ }^{(1)}$ G. Punzi, ${ }^{(1)}$ D. Quarric, ${ }^{(1)}$ K. Ragan, ${ }^{(10)}$ G. Redlinger, ${ }^{(3)}$ J. Rhoades, ${ }^{(18)}$ M. Roach, ${ }^{(17)}$ F. Rimondi, ${ }^{(19)}$ L. Ristori, ${ }^{(11)}$ T. Rolaly, ${ }^{(10)}$ A. Roodman, ${ }^{(3)}$ A. Sansoni, ${ }^{(5)}$ R. D. Sard, ${ }^{(7)}$ A. SavoyNavarro, ${ }^{(1)}$ V. Scarpine, ${ }^{(7)}$ P. Schlabach, ${ }^{(7)}$ E. E. Schmidt, (1) M. II. Schub, ${ }^{(12)}$ R. Scliwillers, ${ }^{(6)}$ A. Scribano, ${ }^{(1)}$ S. Segler, ${ }^{(1)}$ Y. Seiya, ${ }^{(16)}$ M. Sekiguchi, ${ }^{(16)}$ P. Scstini, ${ }^{(11)}$ M. Shapiro, ${ }^{(6)}$ M. Sheaff, ${ }^{(18)}$ M. Shochet, ${ }^{(3)}$ J. Siegrist, ${ }^{(9)}$ P. Sinervo, ${ }^{(10)}$ J. Skarlia, ${ }^{(18)}$ K. Sliwa, ${ }^{(17)}$ D. A. Smith, ${ }^{(11)}$ F. D. Snider, ${ }^{(3)}$ R. St. Denis, ${ }^{(6)}$ A. Stefanini, ${ }^{(11)}$ R. L. Swartz, Jr., ${ }^{(7)}$ M. Takano, ${ }^{(16)}$ K. Takikawa, ${ }^{(16)}$ S. Tarem, ${ }^{(2)}$ D. Theriot, (1) M. Timko, (15) P. 'Tipton, ${ }^{(9)}$ S. Tkaczyk, (1) A. Tollest rup, ${ }^{(4)}$ G. Tonelli, ${ }^{(11)}$ J. Tonnison, ${ }^{(12)}$ W. Trischuk, ${ }^{(6)}$ Y. Tsay, ${ }^{(3)}$ F. Ukegawa, ${ }^{(16)}$ D. Underwood, (1) R. Vidal, ${ }^{(1)}$ R. G. Wagner, ${ }^{(1)}$ R. L. Wagner, ${ }^{(1)}$ J. Walsh, ${ }^{(10)}$ T. Watts, ${ }^{(11)}$ R. Webb, ${ }^{(15)}$ C. Wendt, ${ }^{(18)}$ W. C. Wester, $111,{ }^{(9)}$ T. Westhusing, ${ }^{(11)}$ 
S. N. White, ${ }^{(13)}$ A. B. Wicklund, ${ }^{(1)}$ II. II. Williams, ${ }^{(10)}$ B. L. Winer, ${ }^{(9)}$ A. Yagil, ${ }^{(4)}$

A. Yamashita, ${ }^{(16)}$ K. Yasuoka, ${ }^{(16)}$ G. P. Yeh, ${ }^{(4)}$ J. Yoh, ${ }^{(4)}$ M. Yokoyama, ${ }^{(16)}$

J. C. Yun, (1) F. Zetti(1)

(1) Argonne National Laboratory, Argonne, Llinois 60439

(2) Brandeis University, Wallham, Massachuselts 02254

(3) University of Chicago, Chicago, Illinois 60637

(4) Fermi National Accelerator Laboratory, Batavia, Illinois 60510

(5) Labomatori Nazionali di Frascati,

Istituto Nazionale di Fisica Nucleare, Frascati, Italy

(6) Marvard University, Cambridge, Massachuselts 02138

(7) University of Illinois, Urbana, Illinois 61801

(8) Nalional Laboratory for lligh Energy Physics (KEK), Tsukuba, Ibaraki 305, Japan

(9) Lawrence Berkeley Laboratory, Berkeley, California 94720

(10) University of Pennsylvania, Philadelphia, Pennsyluania 19104

(11) Istituto Nazionale di Fisica Nuclcare,

University and Scuola Normale Superiore of Pisa, 1-56100 I'isa, Italy

(12) Purdue University, West Lafayelte, Indiana 47907

(13) Rockefellcr University, New York, New York 10021

(14) Rutgers University, Piscataway, New Jersey 08854

(15) Texas A8M Universily, College Station, Texas $778 / 3$

(16) University of Tsukuba, Tsukuba, Ibaraki 305, Japan

(17) Tufts Universily, Medford, Massachusetts 02155

(18) Universily of Wisconsin, Madison, Wisconsin 53706 
VITA

Tirnothy Lce Ilessing was

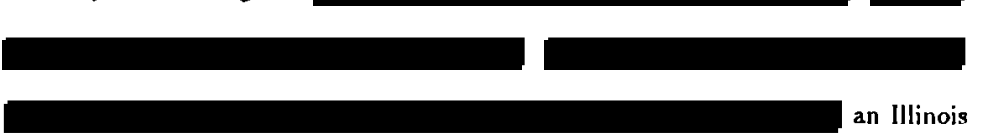

State Scholar and a member of the National Ilous Society. He then attended Augustana College in Rock Island, Illinois, where he earned seven varsity letters while participating in football and wrestling. During this time he was a member of eight College Conference of Illinois and Wisconsin (CCIW) championship teams, including two NCA A Division III National Championship football teauns. In his senior year, he earned All-American Ilonors in wrestling at heavyweight. At Augustana College he inajored in Physics and Math/Computer Science, was inducted into both $\Sigma \Pi \Sigma$, the Physics honor society, and ПME, the Math honor society. Ile graduated cum laude with an A.B. degree from Augustana College in 1985 